Furopean

Surgical Research

Eur Surg Res 2008;40:61-183

Published online: October 30, 2007 DOI: $10.1159 / 0000109878$

The abstracts are only available online, free of charge, under www.karger.com/doi/10.1159/0000109878

\title{
20th National Congress of the Italian Polyspecialist Society of Young Surgeons (IPSYS)
}

November 8-10, 2007, Naples

\section{Abstracts}

\author{
Guest Editors \\ Uberto Andrea Bassi, Napoli \\ Fausto Catena, Bologna
}

Associated Editors

Gabriele Galatà, Rome

Piero Sorrentino, Napoli 


\section{Contents}

Cardiac Surgery $\quad 63$

Transplantation $\quad 66$

Obesity $\quad 71$

Emergency Surgery $\quad 72$

$\begin{array}{ll}\text { Endocrine Surgery } & 77\end{array}$

$\begin{array}{ll}\text { Endoscopic Surgery } & 78\end{array}$

Phlebology $\quad 81$

General Surgery $\quad 84$

Gynecological Surgery 109

Laparoscopic Surgery 110

Maxillofacial Surgery 120

Neurosurgery $\quad 127$

Oncologic Surgery 130

ORL $\quad 136$

Orthopedic Surgery 138

$\begin{array}{ll}\text { Pediatric Surgery } & 143\end{array}$

Plastic Surgery $\quad 146$

Breast Surgery $\quad 151$

Experimental Surgery $\quad 154$

Thoracic Surgery 156

Urology

166

Vascular Surgery 170

$\begin{array}{ll}\text { Author Index } & 178\end{array}$ 


\section{European \\ Surgical Research}

\section{Cardiac Surgery}

\section{1}

\section{Endovascular Repair of Acute Traumatic Injuries of Descending Aorta: Immediate or Delayed Approach?}

\author{
L. Botta ${ }^{1}$, C. Savini ${ }^{1}$, E. Pilato ${ }^{1}$, N. Camurri ${ }^{1}$, E. Mikus ${ }^{1}$, \\ F. Massi ${ }^{1}$, A. Leone1, L. Di Marco ${ }^{1}$, R. Fattori², \\ R. Di Bartolomeo ${ }^{1}$ \\ ${ }^{1}$ Cardiac Surgery and ${ }^{2}$ Cardiovascular Radiology Unit, \\ Cardiothoracovascular Department, University of Bologna, \\ S. Orsola-Malpighi Hospital, Bologna, Italy
}

Introduction: Operative timing and management of acute traumatic aortic rupture (TAR) are still matter of debate. In addiction, no general consensus regarding how best to handle cases with a short proximal neck has been reached. We reviewed our endovascular experience focusing on these topics.

Methods: From July 1998 to September 2006, 27 patients were referred to our Institute for acute traumatic injuries of descending aorta. An early stent-graft procedure was performed in 11 patients (group I), while in 16 patients (group II) endovascular repair (EVAR) was delayed. Median time from trauma was 24 hours (range, 5-120 h) in Group I and 1.5 months (range, $0.8-18 \mathrm{~m}$ ) in Group II. Four patients had an inadequate proximal neck. Of these, 2 had the left subclavian artery (LSA) totally covered by the endoprosthesis and 2 partially.

Results: Technical success was obtained in all patients. There were neither intraoperative nor perioperative deaths. Cerebellar stroke was detected in 1 patient after the intentional closure of LSA. Follow-up (mean, $32.7 \pm 27.5 \mathrm{~m}$ ) was $100 \%$ complete. No late deaths, endoleaks or complications occurred.

Discussion: Endovascular approach was a safe and flexible procedure in TAR and allowed us to fit the operative timing to every single patient's clinical and radiological findings. Intentional closure of the LSA, performed in emergency conditions, is associated to an actuallyumpredictable but effective risk of cerebral ischemic complications. In presence of an inadequate proximal landing zone, conventional open surgery still remains a favourable option as alternative to EVAR associated to surgical revascularization of LSA.

\section{2}

\section{Influence of Preoperative Degree of Aortic Incompetence in Aortic Valve-Sparing Operations}

L. Botta, E. Pilato, N. Camurri, S. Turci, A. Armaro,

M. Bacchini, S.Martin-Suarez, D. Pacini, R. Di Bartolomeo

Cardiac Surgery Unit, Cardiothoracovascular Department, University of Bologna, S. Orsola-Malpighi Hospital,

Bologna, Italy

Introduction: Aortic valve-sparing operations have shown excellent results in patients with aortic root and or ascending aorta aneurysm. Aortic valve regurgitation is frequently detected in these patients as result of significant dilation of the aortic root. The aim of this study is to assess the impact of preoperative aortic incompetence degree on the early and midterm outcomes of sparing surgery.

Methods: From September 2001 to July 2006, 84 patients with aortic root aneurysm underwent aortic valve-sparing surgery according to the reimplantation technique. Depending on preoperative grade of AI, two groups were identified: 31 patients (Study Group, SG) with AI grade III, IV and 53 (Control Group, CG) with AI grade II or less. The Gelweave Valsalva prosthesis was used in 76 patients $(90.5 \%)$. Intraoperative, perioperative variables and outcomes at follow-up were retrospectively compared between the groups.

Results: Patients demographics and types of operations were comparable between groups. Early mortality rates were almost identical in both groups (3.2\% SG vs. 5.7\% CG; p = 0.961); at follow-up, no patient died in both groups. Grade III, IV AI was similar in both groups either early postoperatively $(6.4 \%$ SG vs. $3.8 \% \mathrm{CG}$; $\mathrm{p}=0.981)$ and during follow-up (13.3\% vs. $8 \% ; \mathrm{p}=0.755)$. Reoperation rate for AI was 3.3\% in SG vs. $6 \%$ in CG (p=0.980).

Discussion: Aortic valve-sparing surgery can be safely performed in patients with significant preoperative AI. The reimplatation technique, using the Valsalva conduit, has provided encouraging early and mid-term results and a good reproduction of the pseudosinuses. Moreover patients with satisfactory reconstruction show stable results over time.

\begin{tabular}{ll}
\hline KARGER & ( ) 2008 S. Karger AG, Basel \\
0014-312X/08/0402-0063/\$23.50 \\
$\begin{array}{l}\text { Fax +4161306 12 34 } \\
\begin{array}{l}\text { E-Mail karger@karger.ch } \\
\text { www.karger.com }\end{array}\end{array}$ & $\begin{array}{l}\text { Accessible online at: } \\
\text { www.karger.com/esr }\end{array}$
\end{tabular}




\section{3}

‘No-Touch' Technique Enhance Neuroprotective Effects of Off-Pump Coronary Artery Bypass Grafting

\section{A. Messina ${ }^{1}, 2$, E. Villa', Z. Mhagna ${ }^{1}$, M. Cirillo', F.G. Brunelli', M.D. Tomba', E. Quaini', G. Troise \\ ${ }^{1}$ Operative Unit of Cardiac Surgery, Poliambulanza Foundation Hospital, Brescia; ${ }^{2}$ University of Verona Medical School, Verona}

Introduction: Recent studies have reported neuroprotective effects of off-pump coronary artery bypass grafting (OPCAB). However, only few studies differentiated between OPCAB strategies with or without aortic sideclamp (no-touch technique). Aim of this study is to evaluate the effect of a strategy without aortic manipulation for proximal anastomosis on neurologic outcome in OPCAB.

Methods: Between September 1997 and March 2006, 1,510 consecutive patients with multiple vessels disease underwent complete revascularization with $\mathrm{OPCAB}$ technique (mean age $66 \pm 9$ years; $\mathrm{m} / \mathrm{f} 4: 1$ ). Patients were retrospectively divided into two groups: 635 (42.1\%) with aortic manipulation (Group A) and 875 patients $(57.9 \%)$ without aortic manipulation (Group B). No-touch revascularization was achieved with arterial conduits, arranged as Y-graft or in situ configurations; saphenous vein was employed for the composite graft only in few cases. Neurologic events was defined as clinical evidence of stroke, TIA or RIND.

Results: The graft/patient ratio was $2.9 \pm 0.7$ in group A and $2.0 \pm 0.7$ in group $B(p<0.0001)$. Actuarial freedom from revascularization was similar in both groups at eight years. The incidence of neurologic events was $0.9 \%(n=6)$ in group A vs. $0.1 \%(n=1)$ in group B $(p=0.04)$. Multivariate analysis identified partial aortic clamping to be predictive of stroke $(\mathrm{p}=0.01)$. Carotid disease, history of stroke and diabetes where not significant.

Discussion: Neuroprotective effects of OPCAB is enhanced by absence of aortic manipulation. Surgical strategies aimed to avoid any kind of aortic clamping are strongly recommended.

\section{4}

\section{Atrial Fibrillation after Cardiac Surgery: Comparison of Different Predictive Risk Algorithms}

\author{
E. Angeloni, A. Miceli, S. Refice, T.H. Danesi, \\ U. Benedetto, S. Nardone, B. Fiorani, R. Bianchini, \\ F. Capuano, C. Simon, A. Roscitano, G. Di Nucci, R. Sinatra \\ U.O.C. Cardiochirurgia Ospedale Sant'Andrea, II Facoltà di \\ Medicina e Chirurgia, Università degli Studi di Roma 'La \\ Sapienza'
}

Introduction: Atrial fibrillation (AF) is a common complication after CABG. AF incidence ranges between $10-40 \%$ and is an independent risk factor for cardiovascular and all-cause mortality. There are many predictive risk scores but none has been widely accepted. Among these we compared one developed upon the STS database and a multicenter study by Duke University.
Methods: From May 2004 to August 2007, 573 consecutive patients undergoing isolated CABG were evaluated. The two algorithms were applied to our sample and compared by Receiver Operating Characteristic (ROC) curve. The independent association of postoperative AF was determined using multiple logistic regression.

Results: Postoperative AF incidence was $28.6 \%$. Mean age was $66.8 \pm 9.4$ and off-pump CABG were $17 \%$. The areas under ROC curve were 0.52 and 0.55 respectively $(\mathrm{p}=\mathrm{ns})$. Independent variables were age (OR 1.027; 95\% CI 1.001-1.05; $\mathrm{p}=0.02$ ), preoperative AF history (OR 18.7; 95\% CI 7.9-44.53; $\mathrm{p}<0.001$ ), preoperative use of $\beta$-blockers (OR $0.57 ; 95 \%$ CI $0.38-0.85 ; p=0.06$ ) and off-pump CABG (OR 0.38; 95\% CI 0.2-0.7; $\mathrm{p}=0.002$ ).

Conclusions: We did not find any correlation among these scores and postoperative AF. This may depend on the multifactorial etiology of AF. In this study off-pump surgery and preoperative use of $\beta$-blockers are protective factors, whereas elder age and preoperative AF history are the only independent risk factor.

\section{5}

Previous Percutaneous Coronary Intervention Predicts Low Output Cardiac Syndrome following Coronary Artery Bypass Grafting

\author{
S. Refice, U. Benedetto, A. Miceli, E. Angeloni, \\ T.H. Danesi, G. Sclafani, G. Melina, E. Tonelli, G. Di Nucci, \\ C. Comito, R. Sinatra
}

Cardiochirurgia-ospedale S. Andrea-Roma

Introduction: Previous percutaneous coronary intervention (PCI) is frequently observed in patients undergoing Coronary Artery Bypass Grafting (CABG). Recent studies have suggested that previous PCI is related with increased risk of operative mortality following CABG. However the reason of such a result has to be determined. We hypothesed that previous PCI may be related with increased risk of low cardiac output syndrome (LOS) following CABG.

Methods: A propensity score based analysis of 555 patients undergoing isolated CABG was performed ( 89 with previous PCI and 466 without previous $\mathrm{PCI}$ ). Multivariate analysis including propensity score was performed to investigate risk factors for LOS.

Results: After adjusting for propensity score and covariates multivariate analysis show that previous PCI predicted LOS $(\mathrm{OR}=$ 1.12; $95 \%$ CI: $1.01-1.29 ; \mathrm{p}=0.03)$. Other independent predictors were age $(\mathrm{OR}=1.11$ per year; $95 \% \mathrm{CI}$ : $0.02-1.21 ; \mathrm{p}=0.01)$; male $\operatorname{sex}(\mathrm{OR}=1.3 ; 95 \% \mathrm{CI}: 1.1-1.8 ; \mathrm{p}=0.01)$; emergency $(\mathrm{OR}=1.23$; 95\% CI: $1.12-1.38 ; \mathrm{p}=0.02)$; unstable angina $(\mathrm{OR}=1.6 ; 95 \% \mathrm{CI}$ : $1.12-1.9 ; \mathrm{p}=0.02)$; left ventricular ejection fraction less than 0.30 $(\mathrm{OR}=2.1 ; 95 \% \mathrm{CI}: 1.4-3.1 ; \mathrm{p}=0.02)$.

Discussion: This study showed that previous PCI represents a risk factor for LOS following CABG. This finding may partially explained the increased risk of early mortality in high risk patients. $\overline{64} \quad \overline{\text { Eur Surg Res 2008;40:61-183 }}$ 20th National Congress of the Italian Polyspecialist Society of Young Surgeons (IPSYS) 
6

\section{Risk Factors of Acute Renal Failure in Cardiac Surgery: A Multivariate Analysis}

T.H. Danesi, A. Miceli, S. Refice, E. Angeloni, B. Fiorani,

L. lannone, A. Roscitano, G.D. Di Nucci, R. Sinatra

Cardiochirurgia-ospedale S. Andrea-Roma

Introduction: Acute Renal Failure (ARF) defined by the RIFLE criteria as a reduction of glomerular filtration rate of $50 \%$ occours in $30 \%$ of patients underwent adult cardiac surgery. ARF carries significant morbidity and mortality. Aim of our study is to investigate the risk factors of ARF.

Methods: A total of 928 (mean age $67.8 \pm 10.4$ years) consecutive patients underwent cardiac surgery were included. Exclusion criteria were emergency, preoperative dialysis, monolateral knidney, preoperative chronic renal failure, cardiogenic shock and chronic heart failure. In all patients pre and post-operative craetinine clearance were calculated.

Results: $9.6 \%$ of patients (90/928) developed ARF and 49 of these (54\%) required a CVVHD treatment. Total mortality was $3.2 \%$ Mortality of patients suffered ARF was significantly higher $12 \%$ $(p<0.005)$. Multivariate analysis showed as risk factors associated with postoperative ARF: advanced age $(\mathrm{p}=0.005$; Odds Ratio [OR] 1.13; $95 \%$ confidential interval $[\mathrm{CI}] 1.02-1.27)$, diabetes mellitus $(\mathrm{p}=0.005$; OR $1.13 ; 95 \%$ CI 1.00-1.17), hypertension ( $p=0.004$; OR $1.11 ; 95 \%$ CI 1.1-1.14), reduced preoperative creatinine clearance (Cockroft-Gault algorithm $)(\mathrm{p}=0.004$; OR 1.82; 95\% CI 1.13-1.20), impaired left ventricular function ( $\mathrm{p}=0.002$; OR 1.9 ; $95 \%$ CI 1.02-1.21).

Conclusion: The mortality of patients developing ARF in the post-operative is still high. ARF can be expected in patients with these comorbility.

\section{7}

Use of Minimal Extracorporeal Circulation in Patients Undergoing Myocardial

Revascularization: A Prospective Randomized Pilot Study

\section{J.F. Sciuchetti, F. Formica, A. Martino, V. Giordano, \\ L. Avalli, G. Paolini \\ Cardiac Surgery Clinic, San Gerardo Hospital, University of Milano-Bicocca}

Introduction: To evaluate the early clinical outcome and the biochemical markers with the using of the minimal-extracorporeal circulation (MECC) with Jostra System.

Materials and Methods: 68 patients operated for CABG were prospectively randomized in standard cardiopulmonary bypass $(\mathrm{OCABG}=20)$, MECC System $(\mathrm{MECC}=22)$ and off-pump CABG $(\mathrm{OPCABG}=26) .54$ were male $(79.4 \%)$ with a mean age of $66.6 \pm 10.9$ years. Inclusion criteria were: first cardiac operation, FE $>40 \%$, absence of inflammatory diseases. Myocardial markers were assessed before operation, peri-operatively at 3, 24 and $48 \mathrm{~h}$ after surgery. Data monitored during recovery were: inflammatory response, myocardial damage, hematocrit, clinical outcome.
Results: No deaths occurred in 3 groups. Postoperative increasing of C-reactive protein was no significant among the 3 groups. Postoperative Troponin T was significantly lower in the MECC vs. OCABG group at $24 \mathrm{~h}(0.26 \pm 0.13$ vs. $0.82 \pm 0.15, \mathrm{p}=0.04)$. Moreover, postoperative Creatine kinase-MB level had lower values in the MECC vs. OCABG group and in the OPCABG vs. OCABG group $(p=0.04, p=0.09)$. Lactate level was remarkably lower in the MECC vs. OCABG group and OPCABG vs. OCABG group, 5 minutes after aortic declamping $(1.9 \pm 0.6$ vs. $3.3 \pm 1.0, \mathrm{p}=0.001$ and $1.8 \pm 0.9$ vs. $3.3 \pm 1.0, \mathrm{p}=0.001$, respectively). Low-cardiac-output syndrome occurred in no patients. Intra-operative transfusion rate, length of intensive care unit stay, length of hospital stay and intubation time were lower in the MECC-group than in the other groups.

Discussion: MECC System seems to represent a safe and efficacious surgical approach. It provides a similar outcome of OPCABG and a less incidence of post-operative morbidity and complications, when compared with standard cardiopulmonary bypass.

\section{8}

\section{Extracorporeal Membrane Oxygenation (ECMO) to Support Adult Patients with Cardiogenic Shock. Early Clinical Outcome in a Series of 19 Consecutive Patients}

\author{
J.F. Sciuchetti, F. Formica, A. Martino, L. Avalli, F. Corti, \\ O. Ferro, G. Paolini
}

Cardiac Surgery Clinic, Department of Surgical Science, University of Milano-Bicocca, San Gerardo Hospital Monza, Italy

Introduction: Extracorporeal membrane oxygenation (ECMO) is becoming a validate tool to rescue adult patients with refractory cardiogenic shock. During the last decades, a new generation of oxygenators with a Poly-methylpentene (PMP) membrane are introduced in the clinic. We reviewed our experience with a new PMP oxygenator (Quadrox D) and a centrifugal pump (RotaFlow) which were used to support adult patients with refractory cardiogenic shock.

Materials and Methods: Between January 2000 and April 2007, 19 patients required ECMO for primary or postcardiotomy cardiogenick shock. Mean age was 60.2 years and 12 (63\%) were male. Nine patients $(50 \%)$ suffered primary cardiogenic shock. Cardiopulmonary resuscitation was applied in 12 patients (63\%) with a mean duration time of 33.5 minutes.

Results: Mean ECMO duration time was $7.1 \pm 6.3$ days (range, 1 day to 27 days). Intra-aortic balloon pump was used in 14 patients $(72.2 \%)$ with a mean duration time of $7.7 \pm 5$ (range, 2 days to 17 days). Thirteen patients (68.4\%) survived on ECMO and 6 patients (31.4\%) were discharged. No oxygenators were changed during support because of failure device. Only in one case, the whole ECMO circuit was changed due to persistent sepsis.

Discussion: In our experience with the mechanical support for refractory cardiogenic shock in adult patients, the PMP oxygenator and the centrifugal pump provided acceptable results in terms of surviving on ECMO and discharge. Patients with an initial catastrophic hemodynamic status could benefit by mean of a rapid institution of ECMO with PMP oxygenators. 


\section{Transplantation}

\section{9}

\section{Kidney Transplantation Combined with Other Organs: An Update}

R. Bertelli, B. Nardo, G. Cavallari, G. Ercolani, A. Lauro, G.L. Grazi, P.M. Mikus, E. Pilato, E. Mikus, G. Arpesella,

A.D. Pinna, S. Stefoni, G. Fuga, A. Faenza

Department of Surgery, Intensive Care and

Transplantation, S.Orsola-Malpighi Hospital, University of Bologna

Introduction: We retrospectively reviewed our experience in combined liver-kidney (LKTx) and hearth-kidney (H-KTx) transplantations.

Methods: Between January 1997 April 2007, we performed 25 LKTx and 5 H-KTx. Patients' mean age was $51 \pm 8$ years (LKTx) and $28 \pm 19$ (H-KTx). The main cause of liver failure was chronic viral hepatitis (14). Aetiology of hearth failure was dilatative cardiomyopathy (4) and hypertrophic cardiomyopathy (1). The main causes of renal failure in LKTx were chronic glomerulonephritis (8) and polycystic disease (7). Aetiology of renal failure in H-KTx was interstitial nephropathy (2), vascular nephropathy (2) and chronic glomerulonephritis (1).

Results: Mean follow-up was $32 \pm 26$ months (LKTx) and $24 \pm 17$ months (H-KTx). Immunosuppression was cyclosporine (4) or tacrolimus-based (21) in LKTx; cyclosporine-based in H-KTx. Acute rejection rate was $8 \%$ for both liver and kidney in LKTx; $80 \%$ (mild) for hearth and $20 \%$ for kidney in H-Ktx. LKTx-group: no PGNF and 2 DGF; 1 patient needed postoperative dialysis. One-year graft and patient survivals were both $84 \%$ and overall survival $76 \%$. In H-KTx-group 3 patients needed postoperative dialysis and one cardiac assistance device; overall graft and patient survival was $100 \%$ with good cardiac and renal function.

Conclusions: Our experience confirm H-KTx and LKTx are safe procedures, offering good long term results.

\section{0}

\section{A Multicentric Study on Double Kidney Transplantation}

R. Bertelli', B. Nardo', E. Capocasale², G. Cappelli3,

G. Cavallari ${ }^{1}$, M.P. Mazzoni ${ }^{2}$, L. Benozzi ${ }^{3}$, R. Dalla Valle ${ }^{2}$,

N. Busi ${ }^{2}$, C. Gilioli ${ }^{3}$, A. Albertazzi ${ }^{3}$ S. Stefoni ${ }^{1}$, A.D. Pinna ${ }^{1}$,

A. Faenza ${ }^{1}$

${ }^{1}$ Transplant Center of Bologna; ${ }^{2}$ Transplant Center of

Parma; ${ }^{3}$ Transplant Center of Modena

Introduction: Marginal organs not suitable for single kidney transplantation are considered for double kidney transplantation (DKT).
Methods: Between 2001 and 2007, 68 DKT were performed in the transplant centers of Bologna, Parma and Modena. Recipients' mean age: 62. The main indications were: glomerular nephropathy (28) and hypertensive nephroangiosclerosis (13). Mean HLA A, B, DR mismatches: 3.5. Donors' mean age: 69; mean creatinine clearance: $76 \pm 25 \mathrm{ml} / \mathrm{min}$. The kidneys were almost all perfused with Celsior (mean cold ischemia time: $17 \mathrm{~h}$ ). Mean bioptic score: 4.4. Immunosuppression was tacrolimus (43) or cyclosporine (25) based.

Results: 43 patients had a good postoperative renal function. 25 had acute tubular necrosis; 6 acute rejections occurred. Urinary complications rate was $20 \%$ (11/14 needed surgical revision); there were 6 surgical revisions: intestinal perforation (2), hemorrhage (3) and lymphocele (1). 2 patients lost both grafts due to vascular complications and infective complications. 4 patients underwent transplantectomy of single graft due to vascular complications (2), bleeding (1) and infective complications (1). Graft and patient survival was $97 \%$ and $100 \%, 95 \%$ and $96 \%$ at 3 and 36 months respectively.

Discussion: DKT is a safe way to face organ shortage. The score used in this study is useful to determine whether a kidney should be refused or accepted.

11

\section{Double Laparoscopic Hand-Assisted Donor Nephrectomy: A New Swine Model and Analysis of the Learning Curve}

\author{
G. Cavallari, M. Tsivian, R. Bertelli, F. Neri, G.L. Piras, \\ E. Bianchi, A. Faenza, B. Nardo
}

Dipartimento Discipline Chirurgiche, Rianimatorie e dei Trapianti, Università degli Studi di Bologna

Introduction: The living donor laparoscopic hand assisted nephrectomy (LHAN) diffusion is impaired mainly by the learning curve-associated risks. In this study we create a LHAN swine training model and analyse the learning curve of this procedure.

Materials and Methods: Ten female pigs $(30-35 \mathrm{~kg})$ underwent in the same session a left and then a right LHAN (L-LHAN and R-LHAN respectively). The animals were divided in two groups: group $\mathrm{A}$ - the first 5 animals and group B - the last five animals. In each group we assessed total operative time (TOT), estimated blood loss (EBL), intraoperative complications (IC) and graft quality.

Results: All animals survived bilateral nephrectomy and were euthanized. In two cases (R-LHANs, group A) the procedure was converted due to bleeding. One spleen lesion occurred during trocar positioning and was treated laparoscopically. We registered a decrease and a normalization of the TOT in group B compared to group A $(182 \pm 8$ vs. $245 \pm 44 \mathrm{~min}, \mathrm{p}=0.0176)$. EBL was reduced in group B (21 \pm 6 vs. $44 \pm 30 \mathrm{ml}, \mathrm{p}=0.0484)$.

Discussion: This in vivo training model allows to gain skills in LHAN safely. Moreover, the costs can be sensibly lowered by performing two procedures in each animal and employing reusable instruments. Our model may help transplantation centres in adopting the laparoscopic technique minimizing the learning curve risks.
66

Eur Surg Res 2008;40:61-183 20th National Congress of the Italian Polyspecialist Society of Young Surgeons (IPSYS) 
12

\section{Sirolimus Monotherapy in Liver Transplantation}

\author{
F. Di Benedetto, S. Di Sandro, N. De Ruvo, M. Masetti, \\ R. Montalti, G. Arzu, A. Romano, G. Piero Guerrini, \\ R. Ballarin, M.G. De Blasiis, M. Spaggiari, G.E. Gerunda \\ Università di Modena e Reggio Emilia
}

Introduction: Sirolimus acts by blocking the post-receptor signal transduction of interleukin-2 which interacts with a family of intracellular binding proteins termed immunophilins FKBPs. By this way, SRL can explicate immunosuppressive and antiproliferative action, avoiding nephrotoxicity, neurotoxicity, and diabetogenetic effects as otherwise may occur from calcineurin inhibitors (CI).

Methods: Among 380 liver transplant recipients, 39 underwent immunosuppression switch from CI to Sirolimus monotherapy due to CI adverse effects or because of multifocal HCC beyond Milan criteria or for patients who have developed Kaposi's Sarcoma.

Results: 26 patients were affected by nephrotoxicity from CI. 23 patients showed a significant improvement of renal function after conversion to Sirolimus; 4 patients neurotoxicity with tremor, confusion, agitation, each patient had a complete improvement of symptoms in few days after switch; 1 patient had an untreatable diabetes, and 1 an untreatable arterial hypertension, these patients showed a positive response to the drugs after switch; 3 patients had Kaposi's Sarcoma, 2 of them showed complete remission; and 4 patients were affected from HCC beyond Milan criteria.

Discussion: Sirolimus monotherapy may be considered an effective treatment method to manage CI complication and to control Kaposi's Sarcoma evolution or to prevent HCC recurrence.

\section{3 \\ Outflow Tract Reconstructions in Liver Grafts Injured during Multi-Organ Procurement: Our Experience}

\section{F. Di Francesco, N. Cautero, D. Nicolini, S. De Luca, A. Vecchi, P. Garelli, G. Martorelli, P. Vincenzi, A. Risaliti \\ Chirurgia Epatobiliopancreatica e dei Trapianti di Fegato, Rene e Pancreas, Azienda Ospedaliero-Universitaria 'Ospedali Riuniti', Ancona}

Introduction: The exact frequency and the clinical consequences of surgical hepatic injuries (parenchymal, vascular, or biliary) during organ procurement are unknown. Most of them are related to the presence of many teams during multiorgan procurements or splitting techniques. We describe 2 different cases of suprahepatic-caval outflow reconstruction, in the presence of surgically induced injuries.

Materials and Methods: Case 1: During a combined heartliver organ procurement, the Cardiac Surgery Team wrongly transected the suprahepatic inferior vena cava (IVC) nearness the emergency of the suprahepatic veins of the liver. It was impossible to oversew it or to use a vein patch, to carry out an end-to-side infrahepatic cavocavostomy for the risk to obstruct the outflow of the hepatic vein.
Because of the discrepancy of the size of the iliac vein graft, a useful segment of the infrahepatic IVC from the allograft was transected and used to extend the cuff. Case 2: Erroneous complete longitudinal section of the middle hepatic vein during in situ splitting procedure. An iliac vein graft was used to reconstruct the vein outflow.

Results: In both cases, the implantation was performed successfully by the standard piggy-back technique, without any surgical complication of the vein cuff anastomosis.

Discussion: Although the low rate, induced venous injuries during organ procurements may increase the risk of technical problems during transplantation. An early identification of the damage represent a key point to allow the transplant teams to better anticipate and prepare for surgical repair or reconstructions during the back table.

\section{4 \\ Biliary Complications in Cadaveric Orthotopic Liver Transplantations: A Single Centre Experience}

A. Giuliani, V. Scuderi, W. Santaniello, A. Ceriello, F Sicoli, G.N. Monti, M. Defez, G. Aragiusto, A. Masi, F. Calise

Hepato Biliary Surgery Liver Transplant Centre, Cardarelli Hospital Naples, Italy

Introduction: Biliary tract complications $(\mathrm{BC})$ are reported in $5.8-28.3 \%$ of Cadaveric Orthotopic Liver Transplantation (OLT). The aim of this study is to review our experience on reconstruction of the biliary tract.

Study Type: 153 patients underwent OLT from January 2000 to August 2006. Patients are stratified into two group: group I from January 2000 to July 2004 ( 80 patients) had biliary reconstruction with end to end choledoco-choledoco anastomosis (EECC) either with either without T-Tube; group II from August 2004 to August 2006 (73 patients) had biliary reconstruction with EECC without T-Tube. We analyzed only the patients with a follow-up of at least of 12 months. There were not statistical differences between the two groups regarding ethiology, age, gender, UNOS status and immunosuppressive regimen.

Results: The overall rate $\mathrm{BC}$ was $7.2 \%$ (11/153) including $8.8 \%$ $(7 / 80)$ in group I and 5.5\% (4/73) in group II. The BC in group I were 4 anastomotic strictures treated with endoscopic stent in 3 patients and reconstruction with Roux-en-Y choledocho-jejunostomy in 1. $1 \mathrm{~T}$-Tube dislocation leading to anastomotic kinking treated with endoscopic stent. 1 partial dehiscence of EECC treated with reconstruction with Roux-en-Y choledocho-jejunostomy; 1 biliary leak after T-tube removing treated with surgical suture. The BC in group II were 2 anastomotic strictures treated with endoscopic stent and 2 stricture of recipient bile duct treated with endoscopic sfinterotomy leading to life threatening necrotic pancreatitis in one.

Conclusion: The EECC without T-Tube seems to be the best reconstruction. In our series in the first group 4 surgeons performed anastomosis while in the second group only 2 surgeons performed it, always with the same technique. 
15

\section{A Six-Year Italian Experience in Clinical Intestinal and Multivisceral Transplantation}

\author{
A. Lauro ${ }^{1}$, C. Zanfi ${ }^{1}$, M. Cescon ${ }^{1}$, A. Dazzi ${ }^{1}$, G. Ercolani ${ }^{1}$, \\ T. Kimura1, L. Golfieri', G.L. Grazi', G. Ramacciato', \\ M. Vivarelli', M. Del Gaudio', M. Ravaioli' ${ }^{1}$, A. Cucchetti', \\ G. La Barba1', M. Zanello', G. Vetrone', F. Tuci', \\ M. Di Simone ${ }^{1}$, T. Lazzarotto ${ }^{4}$, A. D'Errico ${ }^{3}$, S. Faenza ${ }^{1}$, \\ L. Pironi ${ }^{2}$ e A.D. Pinna ${ }^{1}$
}

${ }^{1}$ Liver and Multiorgan Transplant Unit (Dir.:Prof. A.D

Pinna), ${ }^{2}$ Chronic Intestinal Failure Center (Head: Prof. L. Pironi), ${ }^{3}$ Pathology 'F. Addarii' Institute, ${ }^{4}$ Microbiology Unit, University of Bologna - S. Orsola-Malpighi Hospital

Introduction: To report the experience in intestinal transplantation in an single European Center.

Patients and Methods: Between December 2000 and May 2007, we performed 38 intestinal transplants in 37 adult patients: 29 isolated intestinal and 9 multivisceral ( 5 with liver). In 3 cases abdominal wall transplant was added. Underliyng diseases were mainly represented by short bowel sindrome (15 patients), chronic intestinal pseudoobstruction ( 9 patients) and Gardner Syndrome ( 9 cases). Indications for transplantation were: loss of venous access (16 patients), recurrent sepsis (12 cases), electrolyte-fluid imbalance ( 8 patients); reversible liver dysfunction was present in 19 cases. Immunosuppressive regimens were based on Daclizumab, Tacrolimus and steroids (12 cases) up to 2002, and Alemtuzumab (24 cases) or Thymoglobuline ( 2 cases) combined with Tacrolimus thereafter.

Results: After a mean follow-up of $1043 \pm 758$ days, actuarial 5 -year patient survival is $74 \%$ for isolated intestinal and 33\% for multivisceral transplant $(\mathrm{p}=0.0029)$, while 5 -year graft survival is $70 \%$ for isolated intestinal and $33 \%$ for multivisceral transplant $(p=0.008)$. The main cause of death was sepsis $(63 \%)$, the main cause of graftectomy was untractable rejection (50\%). Among the 25 recipients alive, $75 \%$ has a normal bowel function with a regular diet without parenteral support, while 6 patients are on parenteral nutrition (1 patient because was recently transplanted).

Discussion and Conclusion: In our series, isolated intestinal transplant has acceptable results in terms of patient and graft survival. In cases of pre-transplant liver dysfunction associated to parenteral nutrition, timely isolated intestinal transplant can prevent the progression to liver failure.
16

\section{Thymoglobuline Use in the Treatment of Intestinal Acute Cellular Rejection Resistant to Traditional Immunosuppressive Therapy}

A. Lauro', C. Zanfi', M. Cescon'1, A. Dazzi', G. Ercolani', T. Kimura ${ }^{1}$, A. Cucchetti ${ }^{1}$, L. Golfieri', G.L. Grazi ${ }^{1}$, G. Ramacciato', M. Vivarelli', M. Del Gaudio', M. Ravaioli', G. La Barba1, M. Zanello', G. Vetrone1, F. Tuci' ${ }^{1}$, A. D'Errico ${ }^{3}$, F.W. Grigioni ${ }^{3}$, L. Pironi ${ }^{2}$ e A.D. Pinna ${ }^{1}$

${ }^{1}$ Liver and Multiorgan Transplant Unit (Dir.:Prof. A.D. Pinna), ${ }^{2}$ Chronic Intestinal Failure Center (Head: Prof. L. Pironi), ${ }^{3}$ Pathology 'F. Addarii' Institute, University of Bologna S. Orsola-Malpighi Hospital

Introduction: To evaluate the use of Thymoglobuline in the treatment of intestinal acute rejection resistant to conventional therapy.

Methods and Results: We treated with Thymoglobuline 4 cases of rejections in 3 adult patients transplanted with isolated intestinal or multivisceral graft. Immunosuppressive therapy was based on Campath 1-H as induction and Tacrolimus as maintenance without steroids. The first recipient had 2 moderate rejections, resistant to conventional therapy (increasing FK, steroids, aspecific immunoglobulins and Campath): administration of 2 doses of Thymoglobuline allowed the complete resolution of rejection. After 2 months, a second episode of rejection forced us to administer again 2 doses of Thymoglobuline, with rapid resolution. The patient developed an Aspergillus pneumonia, 2 bacterial infections (blood and urine), severe uveitis and Pseudomonas and Aspergillus meningitis. She died because of cerebral bleeding. The second recipient developed a moderate rejection resistant to conventional therapy: 6 consecutive doses of Thymoglobuline, with daily bioptic graft control, allowed the resolution of rejection. The patient developed a central line infection, which resolved with antibiotic therapy. The third recipient developed a moderate rejection resistant to increasing of FK, steroids, aspecific immunoglobulins and sirolimus: 6 consecutive doses of Thymoglobuline allowed complete remission. She developed esophageal candidiasis and S. Epidermidis and E. Coli blood infections, which were successfully treated with antibiotic therapy i.v. All 3 patients developed an EBV infection, which was treated with Ganciclovir i.v.

Discussion and Conclusion: Refractory rejection can be successfully treated with Thymoglobuline, but an increased risk of fatal infectious complications even late after transplant is expected. 


\section{7 \\ ACR Monitoring after Intestinal Transplant: Utility of Serological Markers and Zoom Videoendoscopy as Support of Conventional Biopsy and Clinical Findings}

\author{
A. Lauro ${ }^{1}$, A. Altimari², A. Dazzi ${ }^{1}$, C. Zanfi ${ }^{1}$, Z. Miklosova', \\ B. Corti ${ }^{2}$ M. Di Simone ${ }^{1}$, A. D'Errico ${ }^{2}$, A.D. Pinna ${ }^{1}$ \\ ${ }^{1}$ Liver and Multiorgan Transplant Unit, ${ }^{2}$ Pathology \\ 'F. Addarii' Institute, St. Orsola-Malpighi Hospital, \\ University of Bologna, Italy
}

Introduction: Up to now acute cellular rejection (ACR) episodes of intestinal transplant recipients are treated on histological and clinical findings.

Methods and Results: We have applied zoom video endoscopy (ZVE) and use of serological markers as Granzyme B (GrB) and Perforin (PrF) for monitoring of rejection together with the conventional tools. A total of 782 blood samples (obtained at the time of the biopsy) were collected from 34 recipients and $\mathrm{GrB} / \mathrm{PrF}$ up-regulation was recorded among $64.9 \%$ of ACR's during a three years follow-up and, considering only first year post-transplantation, it reached $73.1 \%$ of rejection events. Zoom videoendoscope was used by our group in 29 recipients of isolated intestine $(\mathrm{n}=24)$ and multivisceral transplant $(n=5)$, to enable observation of villi and crypt areas: on more than 270 procedures, $84 \%$ of the zoom findings agreed with histological results, with a specificity of $95 \%$. In fact, during an ongoing ACR, villi were altered in $80 \%$ of cases.

Conclusions: Both procedures can be helpful in supporting conventional histological findings and clinical symptoms of ACR's in intestinal transplant recipients.

\section{8}

\section{Baterial Translocation in Adult Intestinal and} Multivisceral Transplantation
A. Cucchetti ${ }^{1}$, A. Lauro ${ }^{1}$, C. Zanfi ${ }^{1}$, Z. Miklosova' ${ }^{1}$, R. Spiritoso ${ }^{1}$, M. Cescon ${ }^{1}$, A. Dazzi', G. Ercolani ${ }^{1}$, T. Kimura ${ }^{1}$, L. Golfieri', G.L. Grazi', M. Vivarelli', M. Del Gaudio', M. Ravaioli', G. La Barba1', M. Zanello1, G. Vetrone', F. Tuci', M. Di Simone1, A. Bagni ${ }^{3}$, S. Faenza ${ }^{1}$, A. Siniscalchi ${ }^{1}$, L. Pironi ${ }^{2}$ e A.D. Pinna ${ }^{1}$
${ }^{1}$ Liver and Multiorgan Transplant Unit (Dir.:Prof. A.D. Pinna), ${ }^{2}$ Chronic Intestinal Failure Center (Head: Prof. L. Pironi), 3Pathology 'F. Addarii' Institute, University of Bologna - S. Orsola-Malpighi Hospital

Introduction: The application of small bowel transplantation (SBTx) is limited by the relatively high rate of infectious complications that occurs; bacterial translocation (BT) has been suggested as being responsible for the high rate of infections associated with clinical transplantation.

Materials and Methods: We reviewed 95 biopsies performed on 28 isolated SBTx recipients in order to identify histological features predictive of BT within the first month after transplantation.

Results: At least one episode of BT was observed in 13 patients out of 28 (46.4\%); occurrence of BT led to higher one-year mortality
(38.5\%) in comparison to negative cases $(6.7 \% ; p=0.041)$. No clinical or surgical factors were found to be significantly related to occurrence of BT. Of the 95 biopsies examined, 38 were followed by BT $(40 \%)$ : these biopsies showed a higher degree of mucosal vascular alteration (Ruiz grade; $\mathrm{p}<0.05$ ) and ischemia/reperfusion injury (Park/Chiu grade; $\mathrm{p}<0.05$ ) in comparison to negative cases; the presence of acute cellular rejection was not found to be related to BT.

Conclusion: BT seems to be closely related to mucosal vascular alteration and ischemia/reperfusion injury rather than acute cellular rejection; the measurement of the degree of these histological features could identify patients at high risk of potentially life-threatening infectious complications and death.

\section{9}

\section{Psychological Adaptation of Adult Intestinal} Transplant Recipients
L. Golfieri', A. Lauro ${ }^{1}$, E. Tossani ${ }^{2}$, L. Sirri², A. Dazzi ${ }^{1}$,
C. Zanfi ${ }^{1}$, A. Vignudelli ${ }^{1}$, A. Amaduzzi ${ }^{1}$, M. Zanello',
G. Vetrone1, A. Cucchetti', G. Varotti', G. La Barba1,
F. Pezzoli', G. Ercolani ${ }^{1}$, M. Vivarelli ${ }^{1}$, M. Del Gaudio ${ }^{1}$,
M. Ravaioli', M. Cescon, G.L. Grazi', S. Grandi², A.D. Pinna
Liver and Multiorgan Transplant Unit 'F. Addarii' Institute,
St. Orsola-Malpighi Hospital, University of Bologna, Italy

Introduction: Intestinal transplantation has become an accepted therapy for individuals permanently dependent on parenteral nutrition (TPN) with life-threatening complications. Quality of life and psychological well-being can be seen as an important outcome measure of transplantation surgery.

Methods: We evaluated 24 intestinal transplant adult recipients and 24 healthy subjects. All subjects were administered the Italian Version of a Psychological Well-Being Scales (PWB) the World Health Organization Quality of Life-Brief (WHOQOL) and the Symptom Questionnaire (SQ), a symptomatology scale. Quality of life and psychological well-being were assessed in transplant recipients in relation to rejection number, admission number and immunosuppressive protocol.

Results: Transplant recipients reported significantly higher scores in the 'Somatic symptom' $(\mathrm{p}=0.027)$ and 'Hostility' $(\mathrm{p}=0.018)$ dimensions of SQ, compared to control. Transplant recipients reported significantly higher scores in the 'Personal growth' $(\mathrm{p}=0.036)$ and lower scores in 'Positive relation with others' $(p=0.013)$ and in 'Autonomy' $(p=0.007)$ dimensions of PWB, compared to control. In the WHOQOL the scores of transplant patient are lower only in the psychological domain $(\mathrm{p}=0.011)$. Transplant recipients with admission number $>8$ reported higher scores in 'Anxiety' ( $p=0.019)$ and 'Depression' ( $p=0.021)$ scales of SQ and the patients on daclizumab protocol reported higher scores in 'Depression' $(p=0.000)$ and 'Somatic symptom' $(p=0.008)$ dimension of SQ. There aren't significant differences about rejection number and socio-demographic variables.

Conclusion: Improvement of PWB in transplant population may be related to the achievement of the goal of transplantation: recovery of bowel function. But the data confirm that the transplant experience require a long and difficult adaptation trial to the new condition of 'transplant recipient'. 
20

Liver Transplantation with Organ from Acute Methanol Poisoned Donor: Our Experience and Literature Review

\author{
D. Nicolini ${ }^{1}$, N. Cautero ${ }^{1}$, F. Di Francesco ${ }^{1}$, S. De Luca', \\ A. Vecchi ${ }^{1}$, P. Garelli ${ }^{1}$, C. De Angelis ${ }^{2}$, C. Piangatelli², \\ G. Martorelli ${ }^{1}$, P. Vincenzi ${ }^{1}$, A. Risaliti ${ }^{1}$
}

${ }^{1}$ Chirurgia Epatobiliopancreatica e dei Trapianti di Fegato,

Rene e Pancreas; ${ }^{2}$ U.O. di Anestesia e Rianimazione, Azienda

Ospedaliero- Universitaria 'Ospedali Riuniti', Ancona

Introduction: Reported survival rate of liver transplantation (OLT) recipients with organs procured from donors deceased for methanol intoxication (MI) does not differ from that of the patient transplanted from other donors. We report a case of OLT from an methanol (MTH) poisoned donor.

Materials and Methods: A 30 years old female deceased for MI has been evaluated as potential multiorgan donation. At the time of cerebral death a severe metabolic acidosis was present $(\mathrm{pH}<7.0)$ with methanol blood level of $35 \mathrm{mg} / \mathrm{dl}$. The liver was harvested with a normal macroscopic appearance and a $20 \%$ macrovacuolar steatosis and OLT was performed on a HCC patient with HCV cirrhosis in standard piggy-back technique with an immunosuppressive regimen based on steroids, cyclosporine and everolimus.

Results: Liver function test was normal after 7 days and the patient was discharged on postoperativa day 15 . No surgical complications or rejection episodes were observed ant the recipient is currently alive with good liver test after 5 months.

Discussion: Toxicity of MTH is mediated by formic acid, which cause severe metabolic acidosis and lesions to the central nervous system, In Literature has been described only 12 succesfull cases of OLT from donors deceased for MI demonstrating that the MET toxicity is not transmitted to the recipients. According to the literature, in our isolated experience, OLT with a liver from a donor deceased for MI provided an excellent resul without any additional risks for the recipient safety.

\section{1 \\ Colistin: The Re-Emerging Antibiotic for Transplant Recipients}

F. Panaro, F. Piaggio, G. Barabino, C. Ferrari, F. Ravazzoni, E. Andorno, N. Morelli, G. Bottino, M. Casaccia, U. Valente

San Martino University Hospital, Division of General

Surgery and Transplantation, Genoa, Italy

Introduction: Increasing multidrug resistance (M.R.) in Gramnegative bacteria, especially in the transplant recipients, presents a new challenge. Limited therapeutic options have forced infectivologists to reintroduce colistin, an antibiotic discovered in 1950.

Methods: Patients who were hospitalized in our ICU from Jan to Dec 2005 and received intravenous colistin for more than $72 \mathrm{~h}$ were further analyzed. The primary outcome measure was the in-hospital mortality; secondary end points were the clinical outcome of the infections and the occurrence of colistin toxicity.
Results: Five (4 Liver and 1 Kidney-Pancreas Tx) patients received intravenous colistin with a median daily dose of 3 million IU for 24.2 (8-35) days for the management of 9 episodes of infections due to M.R. Gram-negative bacteria. The predominant infections were pneumonia/ sepsis (3), sepsis (2). The responsible pathogens were P. aeruginosa (4) and K. pneumoniae (1). In-hospital mortality was $60 \%(3 / 5)$.

Discussion: M.R. P. aeruginosa and K. pneumoniae are increasingly causing nosocomial infections; New beta-lactamases and carbapenemases are emerging, leading to M.R. strains. Treatment options for M.R. P. aeruginosa and K. pneumonae infections are limited in most cases to carbapenems. In our series, renewed use of an old drug, such as colistin, was the only treatment option for M.R. bacteria.

Conclusions: In the battle against rapidly emerging bacterial resistance we can no longer rely entirely on the discovery of new antibiotics; we must also pursue rational approaches to the use of older antibiotics such as colistin.

22

Protection against Ischemia Reperfusion Injury of an Isolated Perfused Swine Liver with Biliverdin, Carbon Monoxide and Both

\author{
V. Scuderi', G. Aragiusto', A. Bracco ${ }^{2}$, A. Ceriello', \\ A. Giuliani', P. Maida1, M. Romano1, B. Andria², \\ E. Alimenti ${ }^{2}$, D. Morelli², F. Calise ${ }^{1}$ \\ 'Liver Transplantation Unit, 'Antonio Cardarelli' Hospital, \\ Naples, Italy; ${ }^{2}$ Centre of Biotechnologies, 'Antonio \\ Cardarelli' Hospital, Naples, Italy
}

Introduction: Ischemia reperfusion injury (IRI) in organ transplantation remains a serious problem. Heme oxygenase-1 (HO-1) and its products are accepted as likely candidates effectively to treat IRI. In this study we tested $\mathrm{CO}$ and BV for their effects on IRI of the isolated and perfused pig liver, as a relevant pre-clinical animal model.

Methods: Liver were harvested from pigs (donor) weighing 20-25 kg, kept for 18 hours of ischemia in cold Celsior ${ }^{\circledR}$ and subsequently perfused by extracorporeal circulation with a ventilated pig (reservoir) weighing $30-35 \mathrm{~kg}$. The ex vivo liver perfusion was performed for a period of 12 hours. Four groups of pigs $(n=3)$ were used: (1) control group (sham treatments); (2) BV group (both donor and recipient pigs were administered a single bolus of biliverdin $-50 \mu \mathrm{mol} / \mathrm{kg}-2 \mathrm{hrs}$ prior to surgery; (3) CO group (both donor and recipients were exposed to $2 \mathrm{hrs}$ of $\mathrm{CO}-500 \mathrm{ppm}$-, until carboxyhemoglobin levels $15-20 \%$, prior to surgery (4) CO and BV group (recipients and donors were treated with $\mathrm{CO}$ and $\mathrm{BV}$ in combination). Urea synthesis, ammonia clearance and lactate production were evaluated taking blood samples from both the inflow and outflow of the isolated liver. Also bile output was recorded. Liver biopsies were collected from each liver before ischemia, after cold ischemia and 12 hours after reperfusion.

Results: Dual-treatment significantly improved bile production and liver functions compared to the untreated group and $\mathrm{CO}$ or $\mathrm{BV}$ monotherapy over the $12 \mathrm{hrs}$ reperfusion period. Immunohistochemical and TUNEL analysis showed that monotherapy and dual-treatment significantly suppressed the apoptosis after 12 hours of reperfusion.

Discussion: This study shows that in a large animal model of prolonged IRI the addition of $\mathrm{CO}$ and $\mathrm{BV}$ provides, with different mechanisms of action enhanced protection against tissue inflammation and cell death and may potentially be used in future trials in man. 
23

\section{Improved Early Function of Liver Graft by Treatment with N-Acetylcysteine: Feasibility Study}

P. Garelli, A. Vecchi, S. De Luca, N. Cautero, F. Di Francesco,

D. Nicolini, P. Vincenti, G. Martorelli, A. Risaliti

Clinica di Chirurgia Epatobiliare e Trapianti di Fegato, Rene e Pancreas; Azienda Ospedali Riuniti Umberto I-

G.Lancisi-G.M.Salesi- Ancona

Experimental and clinical observations demonstrate that release of oxidative free radicals induced by ischemia and reperfusion (I/R) may produce deleterious effects in liver graft function. The primary graft dysfunction may be an effect of a severe deterioration of the liver microcirculation. N-acetylcysteine (NAC), an anti oxidant and a glutathione precursor, which has been shown to benefit patients with fulminant hepatic failure and ameliorate the detrimental effects of $I / R$ during transplant. The aim of our initial feasibility study is to investigate the effect of NAC for prevention primary non function of suboptimal liver grafts. The experimental donor liver group received NAC $(150 \mathrm{mg} / \mathrm{kg})$ during standard aortic cold flushing during organ procurement, at back table surgery and during preperfusion flushing via the portal vein. The control group did not receive any other drug than standard perfusion. In NAC group versus control group an average of prothrombin time, international normalized ratio, aspartateaminotransferase and alanine-aminotransferase blood level was better. These results although without any statistical significance show a quite better biochemical profile in the NAC group. The administration of NAC was safe without any effects in terms of toxicity, morbidity, mortality and incidence of rejection in both groups. Our local Ethical Committee required this feasibility initial experience to assess the safety profile of NAC and permit the second morpho-hystological phase of the study in a further cohort of suboptimal donors.

\section{Obesity}

\section{4 \\ Laparoscopic Gastric By Pass for Morbid Obesity: Which Type of Anastomosis? Our Experience}

\author{
P. Millo, A. Loffredo, R. Brachet Contul, M. Fabozzi, \\ M. Nardi Jr, J. Da Broi, A. Usai \\ Chirurgia Generale, Ospedale Regionale, Aosta
}

Background: To show our experience in feasibility, indications and limits of different types of laparoscopic gastrojejunal anastomosis in LGBP.

Methods: Between October 2000 and September 2006 one surgeon performed 286 consecutive LRYGBP, 87 mechanical anastomosis according Gagner's procedure (A), 109 using Kockerling Forceps
(Storz) (B) and 70 robotic anastomosis with Da Vinci System (C), 20 hand sewn (D).

Results: Mean operative time was $132 \min (80-420)$. The mean hospital stay was 8 days. Complications related to the anastomotic techniques were: 10 anastomotic bleedings $(3.4 \%)$ that required transfusions: 4 in A, 6 in $\mathrm{B}$ and 0 in $\mathrm{C}$ and $\mathrm{D} ; 22$ marginal ulcers $(7.6 \%)$ : 2 in A, 11 in B, 7 in C, 2 in D; 9 strictures (3.1\%): 1 in A, 7 in B, 1 in C, in D. 4 port site infections in A, 2 in B (1 case in Fobi procedure) and 0 in $\mathrm{C}$ and $\mathrm{D} ; 2$ early incisional hernia in A, 1 case in $\mathrm{B}$ and 0 in $\mathrm{C}$ and $\mathrm{D}$. fistula (1.04\%) in the entire series but 1 fistula was observed in A, 2 in C and 0 in B-D. Mortality rate was zero.

Conclusions: The results show that all types of gastro-entero anastomosis are safe, feasible and reproducible; anastomosis with laparoscopic pursestring is usefull to avoid damage in the oesophagus and robotic anastomosis is precise and less related complications, more sure is confirmed to be hand sewn anastomosis according Higa experience.

\section{5}

\section{Laparoscopic Magenstrasse and Mill Operation}

P. Millo, R. Brachet Contul, M. Fabozzi, A. Usai, M. Nardi, J. Da Broi, U. Parini

Chirurgia Generale, Ospedale Regionale, Aosta

This procedure was performed by Davis Johnston 6 in 1987 and the technique was optimized over time. In this purely restrictive procedure a non banded vertical gastroplasty is created along the lesser curvature without removal of the unused stomach remnant. From the surgical technique standpoint the difference is that after the creation of the access to the lesser sac by means of a window in the gastro-colic omentum, a circular stapler is used to create a window in the gastric antrum, just beyond the incisura angularis, 5 or $6 \mathrm{~cm}$ from the pylorus. An ENDO GIA ä 60 green cartridge is used to divide the body and the fundus along the $48 \mathrm{Fr}$ calibration tube up to the Hiss angle like a long VBG. The free stapled edges are oversewn with a running absorbable suture to reinforce the staple-line, to ensure hemostasis and prevent fistulization between the gastric tube and the separated body of the stomach. In alternative it is possible to buttress the staple line with Seamguard or with bovine pericardium Peri-strips. In literature there are only 2 reports about this procedure and only in open surgery. We have performed some laparoscopic cases without any mortality and morbidity. Satisfactory weight loss is seen at 1 year with $58 \%$ of EWL and $61 \%$ at 2 years. 6,29 . This series describes the possibility to perform a RYGBP as a second step as we describe for SG.

\section{6}

\section{Laparoscopic Sleeve Gastrectomy}

P. Millo, R. Brachet Contul, M. Fabozzi, A. Usai, M. Nardi, J. Da Broi, U. Parini

Chirurgia Generale, Ospedale Regionale, Aosta

Sleeve Gastrectomy is a restrictive, irreversible bariatric operation in which the stomach is reduced to a narrow tube. After a general 
inspection of the abdominal cavity we proceed by measuring $5-7 \mathrm{~cm}$ from the pylorus. In this point we start the division of the greater curvature vascular supply with the short gastric vessels with the LigaSure Vessel Sealing device up to the GE junction. The antrum is transacted by one firing of linear stapler 45 green cartridge, inserted through the port in the right hypocondrium. A $48 \mathrm{Fr}$ bougie is inserted in the stomach along the lesser curvature until the pylorus to calibrate the resection. A linear articulating stapler $60 \mathrm{~mm}$ with green cartridge is inserted through the port in the right hypocondrium to divide the stomach toward the calibration tube and close to the tube, up to the angle of Hiss with sequential firings. During this maneuver the calibration tube avoids any accidental stenosis or cardio-esophageal junction transection, allowing the creation of a standard size gastric pouch $(\sim 150 \mathrm{ml})$. A running suture is placed over the staple line for haemostasis and prevention of leaks. The resected stomach is extracted with a $15 \mathrm{~mm}$ specimen retrieval bag (Endobag). A methylene blue test is performed to check fistulas. A drain is left along the section line and a naso-gastric tube is inserted in the stomach by the anesthesiologist.

\section{7 \\ Roux-en-Y Gastric By Pass (RYGBP) for Morbid Obesity: Laparoscopic Purse String Technique for Gastro-Jejunostomy}

P. Millo, R. Brachet Contul, M. Fabozzi, A. Usai, M. Nardi, J. Da Broi, U. Parini

Department of general surgery, Regional Hospital, Aosta, Italy

The aim of this video is to show a new technique of laparoscopic gastro-entero anastomosis with placement of an intracorporeal pursestring device (Kockeling forceps by Storz). The creation of the Roux limb is done from the first loop of jejunum $50 \mathrm{cms}$ distal to the ligament of Treitz. The bowel is divided with the endoscopic linear cutting stapler (endogia 60 vascular). The alimentary limb is mesured with the help of a marked device. An entero-enterotomy is performed $100-150 \mathrm{~cm}$ distal to the future gastroenterostomy using the endoscopic linear cutting stapler (endogia 60). The anastomotic incision is closed with a 2-0 non absorbable continuous suture. A space adjacent to the lesser curvature, $6 \mathrm{~cm}$ far from the angle of Hiss, is cleared through the lesser curvature mesentery to allow the passage of an endoscopic linear cutting stapler for gastric division (endo gia 45). The Kockerling forceps is introduced in the abdomen to perform an intracorporeal purse-string suture on the proximal gastric pouch. The section of the stapled line allow the creation of a gastrotomy. The anvil of the endoscopic circular stapler $(25 \mathrm{~mm})$ is brough through the gastrotomy into the lumen of the gastric pouch and fixed by closing the purse-string suture. The end of the Roux limb is opened, allowing placement of the main shaft of the endoscopic stapler into the lumen. The anvil and the shaft are connected and the stapler is fired. The excess Roux limb is removed by dividing the bowel with an endoscopic linear cutting stapler.
28

\section{Robotic Roux-en-Y Gastric By Pass (RYGBP)} for Morbid Obesity in Super Obese Patient

P. Millo, R. Brachet Contul, M. Fabozzi, A. Usai, M. Nardi, J. Da Broi, U. Parini

Department of general surgery, Regional Hospital, Aosta, Italy

The creation of the Roux limb is done from the first loop of jejunum $100 \mathrm{~cm}$ distal to the ligament of Treitz. The bowel is divided with the endoscopic linear cutting stapler (endogia 60 vascular). The alimentary limb is measured with the help of a marked device. A entero-enterotomy is performed $150 \mathrm{~cm}$ distal to the future gastroenterostomy using the endoscopic linear cutting stapler (endogia 60). The anastomotic incision is closed with a 2-0 non absorbable continuous suture. A windows adjacent to the lesser curvature, $6 \mathrm{~cm}$ far from the angle of Hiss, is made by dissecting the gastro-hepatic ligament to allow the passage of an endoscopic linear cutting stapler for gastric division (endogia 45). The armonic scalpel can be useful for emostatically clearing the area through the lesser curvature mesentery. The stomac is divided towards the angle of Hiss with multiple applications of linear cutting stapler (endogia 60) to perform a vertical calibrated pouch. The end of the Roux limb is placed near the pouch and the Da Vinci system is placed on the patient and the arms positioned. A robotic hand sewn termino-lateral anastomosis is performed with 2 continous resorbables sutures. A drain is placed near the anastomosis.

\section{Emergency Surgery}

29

Urgency Abdominal Surgery in Patients Over 80 Years of age

R. Bertelli, F. Catena, B. Nardo, S. Di Saverio, L. D'Alessandro, A.D. Pinna

Unit of Urgency Surgery, S.Orsola-Malpighi Hospital, University of Bologna

Introduction: The aim of this study was to determine retrospectively the outcome of the elderly who underwent urgency abdominal surgery.

Methods: The study population comprised 61 consecutive patients aged 80 years or elder who underwent urgency surgery for intra abdominal disorders between June 2006 and February 2007. The variables considered in the study were: patients' age, sex, American Society of Anesthesiologists grading, etiology, time from onset of symptoms to surgery, type of operation, perioperative complications, postoperative ICU admission, length of hospitalization, type of lodgings following acute care (long term care institution vs. home discharge). 
Results: Postoperative mortality was $19.6 \%$ and morbidity $36 \%$. Prolonged hospitalization was related to postoperative ICU admission and early postoperative complications. Early morbidity was not related to the variables considered. The variables associated to 30-day mortality were postoperative complications, ASA score, ICU admission and type of operation (gastrointestinal resection).

Discussion: Postoperative complications as ICU admission resulted important factors influencing both time of hospitalization and early survival. Age of patients, etiology and time to surgery were not related to postoperative complications or patient survival. ASA grade remain the most important preoperative criteria to predict postoperative mortality.

\section{0}

\section{Intestinal Perforation in Patient with Plastic TB peritonitis (Case Report)}

F. Coccolini, L. Ansaloni, F. Catena, S. Severi, F. Gazzotti, L. D'Alessandro, A. Pinna

Policlinico S. Orsola-Malpighi U.O. Chirurgia d'Urgenza, Bologna

Introduction: Even if it's rare, abdominal primary localization of TB is the sixth most frequent form of extra-pulmonary localization. We present a case of intestinal primary localization with fistula, associated with a plastic TB peritonitis.

Methods: A 53 years-old woman from Eritrea presented with a progressive increase of abdominal volume, hyperpirexy and weight loss for the past five months. TC showed pleural bilateral effusion, ascitis and enlarged mesenteric lymph-nodes. A contrast meal was performed and showed three nodules covered by normal mucosa and brought the suspect of jejunum-colic fistula. One day after contrast meal, the patient presents an acute bowel perforation with peritonitis. A laparotomy was performed and the entire visceral surface of peritoneum was full of yellow-white nodules; since the small bowel was totally encapsulated in a plastic peritonitis, the research of perforation wasn't done. Only the toilette of entire abdominal wall was performed and five drains were placed. After an anti-TB therapy was started.

Results: The perforation closed by itself with the patient's general condition improving.

Discussion: In a case like this, with such a complicated bowel presentation, it could be dangerous to research for the perforation. No attempt should be made to locate it. It exists the risk to damage the adherent intestinal loops and focal fistula may result in the postoperative period. The explorative laparotomy associated to medical anti-TB therapy is an effective treatment.
31

\section{Diagnostic and Operative Laparoscopy in Female Patients with Right Lower Quadrant Pain: Our Experience}

\author{
C. De Martino, A. Salvucci, R. Guarino, G. Capuano, \\ M. Milone, M. Musella
}

Naples 'Federico II' Medical School, Department of Surgery and Orthopaedics, General and Emergency Surgery

Introduction: Other pathology besides appendicitis may be found in patients with right lower quadrant pain, expecially in female patients. This has led some to advocate diagnostic laparoscopy/ laparoscopic appendectomy for all such cases.

Methods: From 2003 to 2006, 35 female patients with right lower quadrant pain were considered eligible to diagnostic laparoscopy. In 21 cases, preoperative diagnosis of acute appendicitis was confirmed. In 8 cases laparoscopy allowed to identify gynecological diseases ( 5 salpingitis, 2 bleeding follicles, 1 endometriosis). In one patient, laparoscopy allowed to find a small bowel perforation and in 5 cases pelvic adhesions were found.

Results: Two explorations were converted to laparotomy: it was necessary for a right colon resection in a patient with complicated appendicitis, and for a complete small bowel examination in the patient with perforation. Morbidity was $5.7 \%$, mortality was $0 \%$. The average length of postoperative hospital stay was 3 days (1-12).

Discussion: Laparoscopy is an effective and relatively atraumatic tool to investigate abdominal cavity. This allows an accurate decision-making, which is especially advantageous in young women who have a high rate of non-appendicular pathologies. Laparoscopy also reduces the rate of unnecessary abdominal exploration while realizing a correct diagnosis of others possible pathologies.

\section{2 \\ Management of Caustic Oesophageal Injuries: The Importance of Surgical Timing \\ G. ${ }^{m o}$ De Stefano, M. Armellino, S. Taglialatela Scafati, C. Cataldo, A. De Masi, U. Robustelli \\ U.O.S.C. Osservazione Polispecialistica e Chirurgia d'urgenza, A.O.R.N 'A. Cardarelli', Napoli}

Introduction: The severity and the site of caustic oesophageal injury depend on the substance ingested, quantity ingested and duration of tissue contact. Acids usually cause coagulative necrosis, leading to eschar formation whereas alkali causes liquefactive necrosis resulting in a deep burn. Treatment varies according to severity of lesions and requires a global evaluation of the patient.

Case Report: A 23-year black man was admitted to our unit referring oral and chest pain, hematemesis, refusal to swallow, stridor and hoarseness. The chest pain was described as 'burning'. Upon clinical evaluation, the patient presented diffuse abdominal pain. Both chest and abdomen x-rays along with abdominal ultrasounds did not reveal any finding worth of note. Toxologic examination was also negative for the common substances. Endoscopy revealed the presence of multiple oropharyngeal haemorragic lesions. The oesophageal 
mucosa was thinned with both multiple erosions and foci of massive necrosis causing perforations (third degree injury). Disepithelizzation of gastric mucosa was also observed. Eventually, the patient underwent surgical intervention. Access to the oesophagus was obtained through a combined cervical and transhiatal approach. There were no signs of involvement of other thoracic structures. A segment of jejunum close to the Treitz ligament showing a caustic ulceration was resected and then reconstructed. Oesophagectomy was performed, with the patient receiving a cervical oesophagostomy and jejunostomy. The postoperative course was uneventful. Parenteral nutrition was administered. Surgical reconstruction of the oesophagus was postponed.

Discussion and Conclusions: Timing is a crucial factor in the management of oesophageal caustic injuries. Patient with caustic injuries with absence of oesophageal perforation are placed in Intensive Care Unit. Formation of strictures can be secondarily managed with stent placement. Third degree injury requires surgical intervention. Lapse of time before intervention, is the most significant factor affecting the outcome. Mortality, in fact, increase as the perforation extends into the thorax causing mediastinitis. Secondary reconstruction is indicated for oesophageal injury due to caustic injury.

\section{3 \\ Mortality and Morbidity Meetings in the Emergency Department}

M. Della Corte

AORNAS, S. Giovanni di Dio e Ruggi D’Aragona, Salerno

Introduction: Mortality and Morbidity meetings not only are a recognized instrument to pinpoint flaws in the assistance offered to patients and to implement corrective strategies but they are also a useful learning tool as they allow a retrospective analysis of real clinical scenarios where possible errors that may have been made or faults in the system can be elicited and discussed with those involved.

Methods: From the year 2001 the notes of all patients who had attended the Emergency Department of the John Radcliffe Hospitals NHS Trust and died within 24 hours have been collected by use of a computerized hospital dead list and a list of patients who had died within 2 hours from arrival (who were not included in the hospital list). Patients who were already dead on arrival were excluded from analysis. Time to call to time of arrival to hospital, vital signs on route, drug administered and other actions taken by paramedics were obtained by analyzing the pre-hospital sheets that are routinely filled in and handed over on arrival by the ambulance crew. ED notes and triage sheets were analyzed looking for time of arrival to time of being seen by doctor, vital signs recorded, times of tests and results, actions taken, times of administration of dugs, time of referral to specialist to time of being actually seen by the specialist (as per hospital notes) and further inpatient management sheets. Also the coroner's report was obtained to confirm the actual cause of death and see how it would compare to the clinical diagnosis. The cause of death and management of all patients were presented at the M\&M meetings on a monthly basis.

Results: The results of a year of data collection and discussion, and the actions taken are presented. As a consequence of the results obtained all the clinicians involved were informed in a non judgmental way of the mistakes and near misses occurred and further training in weak areas was provided, a departmental ward round three times a day with a Consultant was implemented and further specific issues were discussed and corrected.

Discussion: The nature of Emergency Departments offers the unique possibility to analyze the actions and practices of many several specialists often involved in the care of a single patient, in a real life situation and in a rapidly changing and often challenging environment. However, the implementation of a Mortaliy and Morbidity meeting in the Emergency Departments is often made difficult by the fast turnover and high number of attenders and the fact that often their care is passed on to other specialists or discharged and not followedup. We believe that a Mortality meeting can be implemented and used as a tool to improve the management of patients, prevent the recurrence of avoidable errors and offer tailored training to individual clinicians, even in an Emergency Department where patients stay for a very limited time, but where a properly made diagnosis and management plan can mean the difference between life and death.

34

\section{Role of Water-Soluble Contrast (Gastrografin ${ }^{\circledR}$ ) in the Diagnostic and Therapeutic Management of ASIO (Adhesive Small Bowel Intestinal Obstruction): A Prospective Randomized Trial}

S. Di Saverio, F. Catena, L. Ansaloni, M. Gavioli, F. Gazzotti, G. Ercolani, S. Gagliardi, R. Bertelli, A. Lauro, M. Cescon, L. D'Alessandro, A.D. Pinna

General, Emergency, Transplant Surgery Department

S. Orsola University Hospital, Bologna

Introduction: Patients presenting with ASIO are difficult to evaluate and their treatment is controversial. Aim of this study is to determine the diagnostic and therapeutic role of Gastrografin (GG) in patients with ASIO.

Materials and Methods: The primary endpoints of the study were the evaluation of the operative rate reduction and the hospital stay. The first group received traditional treatment (TT: control group) whereas the second group was submitted to GG meal and follow-through study immediately.

Results: 76 patients were randomized in two arms of treatment. In the GG group obstruction resolved subsequently in 31 cases $(81.5 \%)$. The remaining 7 patients were submitted to surgery ( 1 bowel resection). In the control group 21 patients were not submitted to surgery (55\%): 2 patients had bowel strangulation and underwent resection, the remaining 15 presented untreatable obstruction. The difference in the operative rate between the two groups resulted significant $(\mathrm{p}=0.013)$. No complications GG-related nor significant differences in the relapse rate were found. The length of hospital stay revealed a significative reduction (4.6 days for GG vs. 7.8 days for TT $\mathrm{p}<0.05)$. The time from obstruction to resolution of symptoms was significantly lower in the GG group $(6.4 \mathrm{hrs}$ vs. $43 \mathrm{hrs}, \mathrm{p}<0.01)$.

Conclusion: The use of GG in ASIO is safe and reduces the time to resolution of obstruction, the operative rate, as well as the hospital stay. 


\section{5 \\ Intestinal Malrotation with Right Colon Volvulus plus Carcinoid Tumor of the Appendix: A Case of Acute Abdomen in a Young Adult}

\author{
I. Fiume, G. Roina, I. Pisapia, C. Cremone, B. Cardamone \\ ASL SA1 - P.O. Cava de' Tirreni 'S. Maria Incoronata \\ dell'Olmo' - U.O. Chirurgia Generale
}

Introduction: Intestinal malrotation is an embryologic anomaly of intestinal rotation and fixation. Arrest of this development may occur at any stage resulting in a group of major and minor abnormalities in relation to the upset of rotation around the superior mesenteric artery and fixation at the peritoneum. Intestinal obstruction due to volvulus is the commonest presentation in symptomatic cases but the association with carcinoid tumor of appendix is the first report at a pub med search.

Case Report: A 19-year-old female was admitted with abdominal pain, nausea, vomiting, and constipation. Abdominal Rx showed distended small bowel loops with fluid levels suggesting acute intestinal obstruction. A Gastrografin enema showed the entire colon with no contrast filling of terminal ileum. At explorative laparotomy we found a large bowel loop twisted around its long axis constituted of terminal ileum, caecum and proximal colon disposed in 'Omega' shape with the caecum at the lower left abdominal quadrant. In spite of the surgical manoeuvres of derotation, the loop remained distended and atonic, therefore resection followed by an ileal-colonic anastomosis was performed. The histological examination revealed the concomitant presence of an intramural carcinoid of the appendix.

Discussion: Symptomatic patients may present either acutely with bowel obstruction and intestinal ischemia or chronically with vague abdominal pain. In the presented case the nonfixation of the colon during fetal life was the cause of bowel obstruction resulting from right colon volvulus. Aggressive definitive surgical treatment allowed to treat both occlusion, segmental ischemia and the carcinoid of appendix efficaciously without need of further surgery.

\section{6}

\section{Laparoscopic versus Open Appendectomy:} A Single Institution Prospective Study

\author{
G. Giraudo, G. Masi, M.C. Giuffrida, F. Borghi \\ Dipartimento di Chirurgia Generale, Ospedale Santa \\ Croce e Carle, Cuneo
}

Introduction: Laparoscopy in patients with a clinical suspicion of acute appendicitis remains controversial. A prospective study was performed to compare the outcome of laparoscopic (LA) and open appendicectomy $(\mathrm{OA})$ in patients with suspected acute appendicitis (AA).

Methods: Between May 2000 and July 2006, a prospective analysis was performed on 746 patients aged 3 to 90 years that underwent emergency appendectomy for the clinical, ultrasonography or computed tomography (CTscan) findings of AA at our Department.
Patients with suspected AA not confirmed by pathological examination $(23,3.1 \%)$ were excluded.

Results: A total of 723 patients ( 377 males and 346 females, the average age 28.8) were included in the study: 600 patients ( 348 males and 252 females, the average age 27.9) were allocated to OA and 123 patients (94 females and 29 males, the average age 33) to LA, one $(0.8 \%)$ of whom required conversion to open appendectomy. LA took $56.7 \pm 16.6$ minutes, OA took $44.3 \pm 16.5$ minutes $(\mathrm{p}<0.0001)$. Hospital stay days were shorter for the LA (3.52 days versus 4.26 days, $\mathrm{p}<0.05)$. Complication rate $(7.31 \%$ in LA versus $7 \%$ in OA) did not differ between the two groups. Two patients $(0.33 \%)$ died in the OA.

Discussion: Our results indicated that LA is a safe and effective procedure for treating patients with AA. Higher cost is compensated for with shorter hospitalization.

37

\section{Shock Emorragico}

\section{Grassi, G. Bongini, V. Grassi \\ Department of Emergency Montepulciano, USL 7 Siena}

Introduction: It comes in Goddess a man of 62 aa, discharged by around 15 department ggs of surgery $\mathrm{x}$ interv of gasrectomi subtoatle $\mathrm{x}$ etp stomach. In anamnesis paz iperteso, prgresso IMA, ipercolesterolemia. In therapy with atiipertensivo, gastric coverage and lower part ASA dosing.

Material and Methods: - PA $90 \backslash 60 \mathrm{mmHgs}$ - Paz, cold skin, vigilant collaborate of it, he/she remembers what happened. Abundant Ematemesi. EGA: $\mathrm{pCO}_{2}: 33 ; \mathrm{pH}: 7.2 ; \mathrm{HCO}_{3}: 19 ; \mathrm{HB}: 10.0 \mathrm{~g} \backslash \mathrm{dl}$; $\mathrm{SATO}_{2}: 98 \%$.

Treatment: $1,000 \mathrm{cc}$ E, $600 \mathrm{cc}$ blood intero, 1,000 cc RL, CV:50 cc clear urines., SNG: 400 cc dark sague. GB 11.8; GR3, 24; HB: $9.2 \mathrm{~g} \backslash \mathrm{dl}$; HCT: 28.6; Piastrine: 353. ECG: nei limits, pregressa ischemia ant-inf. RX-direct addome:non marks of perforation. Abdominal Ecografia. Modest quantity of picked perisplenica. Dccie free parietocoliche, also the Morrison and the Douglas. Relaxation del'ansa afferente there is full of fluid material. E'presente relaxation of the efferent handle that contains material corpuscolato and it is endowed with vivacious peristalsi. Constituted the team of urgency (physician of the Goddess, anesthetist, endoscopista); EGDS is performed in urgenza: sanguinamento to level of the anastomosi gastrodigiunale. Elettrocoagulazione.

Discussion: The paz is hospitalized in surgery, low after three ggs under discreet conditions clinics. The mechanisms patogeneticis of the shock are: Inadequate (shock ipovolemico) circulatory volume: emorragicoldeplezione of liquids - Compromissione of the vascular (distributive shock) tone: septic, anafilattico, neurogeno, spinal - critical Deficit of the cardiac (shock cardiogeno) course miogeno, mechanic, arrhythmic - Obstacle to the outflow ventricolare (obstructive shock) cardiac tamponamento, pulmonary tromboembolia, pneumotorace.

Conclusion: In our case it deals with a shock emorragico, adequately treated and quickly. 
38

\section{Launch Us Esophageal}

1. Grassi, G. Bongini, V. Grassi

Department of Emergency, Montepulciano, USL 7 Siena

Introduction: A patient (M) of 30 aa, with cirrhosis of the liver $\mathrm{HCV}+$, etilista, affection from launch us esophageal, severe gastropatia, stadium funzionaler child pugh, tuberculous pulmonary lobe sup $\mathrm{dx}$, cardiopatia dilated you. To the objective examination introduces stained suits with alive red blood, pale, algid perspiration, cuore:nei limti, chest: nothing to be signalled, abdomen. Aching palpable liver not, not edemas slopes not turgidity of the jugular ones. PA $80 \backslash 45$, FC 78, Sa O2 100\%. Diuresi: assente.

Material and Methods: Trattato:600 $\mathrm{cc}$ whole (negative zero) blood, Plasma 2 knapsacks, Plaques 2 knapsacks, red Globoli concennnrati 1 sacca, 500 emagel, 2000 ringer lactate. 1 fl solmedrol, logastatina $0.1 \mathrm{fl}$, bicarbonate $250,4 \%+40 \mathrm{mEqs}$ bic of sodium, propofol $200 \mathrm{mgs}$ in bolus esguita IOT Comes. Later around $3 \mathrm{~h} 30 \mathrm{~min}$, PA 105 $\backslash 45$, FC 100, Sat O2 100. Diuresi: $200 \mathrm{ml}$. Examinations instrumental esguiti: Rx-chest. You positions probe of Sengstaken blakemore.

Discussion: The criterions to define a hemorrhage massive and uncontrollable sono:presenza of shock emorragico, conditions unstable emodinamiche, maintained only at the price of continuous transfusions. In this case a conservative therapy has been performed using the Sengstaken-Blakemore xchè it dealt with launch us esophageal bloody. And also true that some types of digestive hemorrhage have tendency to recidivare both to brief that to longer distance of tempo.

Conclusion: In case of multiple recidivists it exists without doubt the indication to the surgical approach. Particularly, in the case of the hemorrhages gastro-duodenali, the indication exists to the intervention if a recidivist is verified during the same refuge.

\section{9}

Acute Small Bowel Bleeding: Report of Three Different Cases

\author{
G. Ioia, C. De Martino, A. Mottola, G. Lombardi, \\ A. Esposito, D. Lombardi \\ Università degli Studi di Napoli Federico II
}

Introduction: Authors report the experience of three unusual cases of acute small bowel bleeding in the young male, describing their clinical presentation, preoperative management and surgical performance.

Methods: Three male patients, aged 25, 38 and 41, were admitted at Emergency Surgery Unit for acute gastrointestinal bleeding. Upper and lower endoscopy did not identify the source of bleeding. This findings pointed our attention to small bowel. The conditions of the three patients did not allow to spend time with capsule endoscopy or nuclear medicine tecniques, so all patients received mesenteric selective arteriography and contrast-enhanced CT.

Results: Arteriography identified the source of bleeding in all cases and was useful to hypotesize its etiology: ileal angio-dysplasia, duodenal stromal tumour and Meckel diverticulum. The patient with bleeding Meckel diverticulum underwent superselective catheterism and embolization. All patients have been operated. Surgical findings and post-operative pathologic analysis confirmed the radiologic diagnosis.

Conclusions: Mesenteric selective arteriography is an effective tool in patients with acute small bowel bleeding: it achieves a correct identification of the source of bleeding and helps to hypotesize its etiology. Embolization reduces bleeding and allows to improve the clinical condition of the patients before surgery.

\section{0 \\ Surgical Management of the Complicated Diverticulosis of the Colon}

L. Sparavigna, M. Mastrorilli, G. Mastrorilli, A. Martini,

A. Maresca

Unit of Emergency Surgery - 'Umberto I' Hospital, ASL Sa1, Nocera Inferiore, Salerno

Introduction: Diverticular disease has increasing incidence in industrialised countries. Conservative or medical therapy is usually indicated for uncomplicated diverticulitis. Indications for surgery include recurrent attacks and complications of the disease. The present study analyzes our results with different types of surgery adopted in the treatment of the complicated diverticulosis of the colon.

Methods: A retrospective study analyzing stage type of complication, the surgical technique adopted, Hinchey stage, mortality and morbility rates and average hospital stay correlated with the kind of intervention has been carried out on 62 surgical interventions performed between 2001 and 2006 in our Hospital.

Results: We have performed 26 primitive anastomosis resection (in the I and II stage), 34 Hartmann's procedure (in the II and IV stage), 2 CT guided percutaneous drainage with subsequent elective anastomosis resection resection (in the I stage). The total mortality rate amounts to $8 \%$; the morbidity rate of the primitive anastomosis resection interventions to $15 \%$, Hartmann's procedure to $11 \%$. Furthermore we have performed 9 reconversation after Hartmann's procedure; in the second operations the mortality and morbidity rate amounts to 0 and the hospital stay to 9 days.

Discussion: According to the data obtained and to current literature, it results that the primitive anastomosis resection represents the first choice intervention in the I-II stage. Hartmann's procedure confirms its effectiveness in the III-IV stage.

41

\section{Gastrointestinal Bleeding in Emergency Care Unit. A Retrospective Study of One-Single Institution}

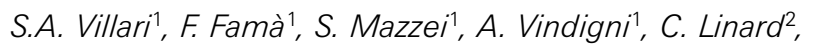
M.A. Gioffrè-Florio ${ }^{1}$

'Division of Emergency Care Unit, University of Messina, Italy; ${ }^{2}$ Service de Anesthésie et Réanimation, Centre Hospitalier Régional, Orléans, France

Introduction: Gastrointestinal (GI) bleeding is a common reason for admission to an Emergency Care Unit and has been 
traditionally associated with a mortality rate of about $5-10 \%$ reported in the literature.

Materials and Methods: In 2005 in the Emergency Care Unit of the University Hospital, 226 patients were observed [138 males (61.1\%), 88 females (38.9\%)], age range 20-99 (mean age 66). Upper and lower GI bleeding was diagnosed with a prevalence of rectorragy (101 cases $-44.7 \%$ ). In the other cases melaena ( 85 cases $-37.6 \%$ ) and hematemesis ( 40 cases $-17.7 \%$ ) were found. All patient were triaged as yellow code and admitted after the stabilization of vital parameters.

Results: Bleeding was identified by colonoscopy in 12 patients and by gastroscopy in 46 cases. The mean emergency room stay was 55 minutes. The causes of bleeding were detected in 147 cases $(65 \%)$. The most frequent etiologies were: erosive gastritis (13\%), gastric $(6.1 \%)$ and duodenal $(11 \%)$ ulcer, gastric $(2.7 \%)$ and bowel $(6.8 \%)$ tumors, diverticulosis $(7.5 \%)$, haemorrhoids $(4 \%)$.

Discussion: In 2005, 39,752 patients were admitted to our emergency ward. GI bleeding was found in $5.7 \%$ of them. Among yellow codes, bleeding is frequently found in emergency units. The prognosis prediction is generally difficult but hypotension $(<90 \mathrm{mmHg})$ lead us to suspect a negative outcome. Chronic oral anticoagulant therapy is associated with an increase in GI bleeding (19.5\%). The endoscopy followed an early evaluation of pathological severity status and it is important for treatment and prognosis.

\section{Endocrine Surgery}

42

\section{Human Parathyroid Cell Proliferation in Response to Different Growth Factors. Preliminary Results of an Experimental Study}

\author{
G. Cavallaro, A. Polistena, G. De Toma
}

Department of Surgery 'P. Valdoni', Policlinico Umberto I, University 'La Sapienza', Rome

Introduction: Parathyroid adenoma is commonly seen in patients affected by primary hyperparathyroidism. As in other kinds of endocrine tumours, cell proliferation and tumour growth appear to be regulated by various growth factors, creating a complex network of stimuli between chief cells and stromal cells. The aim of this study is to determine the effects of different growth factors on cell proliferation, in vitro.

Methods: Human parathyroid adenoma cells, first identified by immunohistochemistry, were put in culture. Growth factor expression was determined by immunohistochemistry. Then different concentrations of these growth factors (bFGF, VEGF, TGF- $\beta$ and IGF-1) were added to the medium, to evaluate their effect on cell proliferation, determined by immunocytochemistry and cytofluorimetry.

Results: Immunohistochemistry showed increased expression of bFGF, VEGF, TGF- $\beta$ and IGF-1 in parathyroid adenomas with respect to normal glands (incidentally removed during thyroid surgery). These growth factors demonstrated to have different effects on adenoma cell proliferation, in vitro. In facts, $14.5 \%$ of TGF- $\beta$ stimulated cells were found in S-fase, while $19.55 \%$ of IGF-1 stimulated cells were found in S-fase, and only $5.58 \%$ of VEGF stimulated cells were found in S-fase.

Discussion: The preliminary results of this study, even if still not supported by statistic significance, try to show and explain the complex network of stimuli responsible of cell proliferation and apoptosis in parathyroid adenoma. This experimental model could even be extrapolated to other kind of tumours, both endocrine and not.

\section{3 \\ Circulating Thyroglobulin mRNA does not Predict Recurrence in Patients who Underwent Thyroidectomy for Cancer}

\author{
C.P. Lombardi ${ }^{1}$, M. Bossola ${ }^{1}$, P. Princi ${ }^{1}$, M. Boscherini ${ }^{1}$, \\ M. Raffaelli', G. Latorre², M. Salvatori3, G. Perotti ${ }^{3}$, \\ A. Pontecorvi ${ }^{4}$, W. Ricciardi ${ }^{2}$, R. Bellantone ${ }^{1}$ \\ ${ }^{1}$ Division of Endocrine Surgery, ${ }^{2}$ Institute of Hygiene and \\ Epidemiology, Biostatistic Unit, ${ }^{3}$ Institute of Nuclear \\ Medicine, ${ }^{4}$ Istituto di Patologia Speciale Medica, \\ Università Cattolica del Sacro Cuore, Rome, Italy
}

Introduction: Aim of the present study was to evaluate if serum thyroglobulin mRNA assay predicts recurrence in patients who underwent thyroidectomy for cancer.

Patients and Methods: Sixty-six consecutive patients operated on between April 1997 and July 1999 were studied. One year after surgery, a blood sample was taken for serum thyroglobulin (Tg) immunoassay and for Tg mRNA assay by reverse transcriptionpolymerase chain reaction (RT-PCR). All patients underwent periodically clinical examination, blood sample for serum thyroglobulin immunoassay, neck ultrasound and radioiodine scan or treatment, if indicated. Kaplan-Meier estimates of survival were calculated according to the presence or absence of circulating Tg mRNA and according to the thyroglobulin levels, at baseline.

Results: Tg mRNA was detected in $14(21.2 \%)$ of 66 patients with thyroid carcinoma. With a median follow-up of 61.7 months (range 54-80), eight patients (12.1\%) relapsed. Among patients with detectable Tg mRNA (n. 14), 1 distant metastases occurred (7\%) while lymph node metastases (n. 3) or distant metastases (n. 4) were detected in 7 out of 52 patients $(13.4 \%$ ) with undetectable Tg mRNA. Tumor relapse occurred in 7 out of 8 patients $(87.5 \%)$ with elevated serum thyroglobulin and only in 1 out of 58 patients $(1.7 \%)$ with normal or undetectable serum thyroglobulin. The disease-free interval of patients positive at baseline for Tg mRNA was similar to that of patients with undetectable Tg mRNA at baseline.

Conclusion: The present study suggests that detection of circulating Tg mRNA in patients who underwent thyroidectomy for cancer does not predict recurrence. 


\section{4}

\section{Decisive Role of Intraoperative Quick Parathyroid Hormone Monitoring (qPTH) in the Treatment of Primitive Hyperparathyroidism (pHPT)}

\author{
A. Tatti, S. Farris, J. Atzeni, A. Melis, P.G. Calò, A. Nicolosi \\ Policlinico Universitario-Dipartimento di Chirurgia, \\ Monserrato, Cagliari
}

Introduction: The $\mathrm{qPTH}$ assay plays an important role in parathyroid surgery, particularly in ectopic glands and multiglandular disease. The accuracy of this method reaches $96-97 \%$.

Materials and Methods: Between Nov 2001 and Jun 2007124 patients (107 females and 17 males, aged 25-79) were operated for pHPT in our department. A single parathyroidectomy was performed in 107 patients; 12 of those $(9.67 \%)$ were ectopic glands. A multiple parathyroidectomy was performed in 17 cases. The qPTH assay was used in 109 patients $(87.9 \%)$; it allowed to find two multi-adenomas and led to multiple parathyroidectomy in cases of hyperplasia (17 patients). It also confirmed an intraparenchymal adenoma after emithyroidectomy (4 patients) and supported the research of the ectopic glands (12 patients). Five patients had a persistent high value of $\mathrm{qPTH}$ and had to undergo more tests and another operation. Histology resulted in 85 cases of 'singular adenoma', 22 of 'hyperplasia', 2 of 'multiple adenoma (double)', 3 of 'carcinoma'.

Conclusions: In 35 cases, the qPTH assay has been basic for the success of the operation and to prevent a persistent $\mathrm{pHPT}$. There were 3 false positives: in two cases they were due to hyperplasia and in one case to a thyroid carcinoma. The qPTH assay has proved to be a safe, easy and effective test which is fundamental for a correct performance of the operation. It has also proved, even in the operating bed, the suitability of treatment and has avoided the persistence of the illness.

\section{5 \\ Indications and Results in Mini-Invasive Video Assisted Thyroidectomy (M.I.V.A.T.)}

G. Docimo, R. Ruggiero, G. Pacca, S. Gili, E. Irlandese, F. Lo Schiavo

Second University of Naples - Surgical Department

Introduction: Mini-invasive surgery of the thyroid via M.I.V.A.T. (mini-invasive video assisted thyroidectomy), after the first applications performed in 1999, with the correct indications, encountered increasing major approval. With this technique it is possible with reduced incisions, inclusive between $2,5-3 \mathrm{cms}$, to perform interventions of lobectomy or total thyroidectomy.

Methods: Since April 2006 we have selected 11 patients for treatment via M.I.V.A.T. We carried out the inclusion criteria as proposed by Miccoli that foresee nodules of diameter $<3 \mathrm{cms}$ with thyroid volume $<30 \mathrm{mms}$, benign lesions or lower grade follicular, ca papillar. This method foresees a first phase, up to the medialisation of the thyroid lobe, according to the traditional technique, thereafter a video-assisted phase up to the exteriorisation of the thyroid lobe and finally the lobectomy is completed via direct vision.
Results: We carried out 7 lobistmectomies and 4 total thyroidectomies with this technique, without having to change technique.

Discussion: Numerous studies have shown similar results in terms of complications between M.I.V.A.T. and open thyroidectomy. In wiew of these results the M.I.V.A.T. has given proof of reduced postoperative pain due to the position of the neck not iperexented on the operating table like in open surgery, with evident reduced cosmetic results. The endoscopic magnification offered by M.I.V.A.T. allows optimal visibility of all the anatomical particulars including the riccorente and the parathyroid, with operating times superimposable compared to those in traditional surgery. Although such procedure still has indications which are limited, we believe that M.I.V.A.T., with the correct indications, is truly an alternative to traditional surgery.

\section{Bibliography}

Miccoli P, Berti P, Raffaelli M, Conte M, Materazzi G, Galleri D: Minimally viedeoassited thrydectomy. Am J Surger 2001;181:567-570.

Materazzi G, Dionigi G, Berti P, Rago R, Frustaci G, Docimo G, Puccini M, Miccoli P: One day thyroid retrospettive analysis off safety and patiente satisfection on a consecutive series of $1571 \mathrm{n}$ cases over a free three yars period. Eur Surg Res 2007;39:182-188.

\section{Endoscopic Surgery}

46

\section{A Camera Handler for Miccoli's Minimally Invasive Video-Assisted Thyroidectomy and Parathyroidectomy Procedures and other Laparoscopic Procedures}

\author{
A. Maura ${ }^{1}$, G. Galatà ${ }^{2}$, F. Rulli1,2 \\ ${ }^{1}$ Innovative Surgical Technologies (Medical Engineering \\ School), ${ }^{2}$ Department of Surgery, University of Rome 'Tor \\ Vergata'
}

Introduction: Minimally invasive video-assisted thyroidectomy and parathyroidectomy (MIVAT/P) are surgical procedures performed with a 5-mm camera handled by a camera assistant. In general, every laparoscopic procedure requires an assistant handling the camera while performing the procedure.

Materials and Methods: The authors created a mechanical articulated arm acting like a camera handler during video-assisted surgical procedures. It consists of two telescopic links, an adjustable support ring and a fixing clamp to the operating table. The joints between links and between the second link and the support ring are spherical on the superior side and hinge on the inferior side; the first link is connected to the clamp by a spherical joint. Every joint has a blocking system to allow fixing in the desired position. The support ring is adjustable in order to handle up to $12-\mathrm{mm}$ cameras.

Results: The device gives, with minimum spatial encumbrance, fast setup and stable fixing of the camera in the operating field, allowing precise movements during the procedure.
78

Eur Surg Res 2008;40:61-183 20th National Congress of the Italian Polyspecialist Society of Young Surgeons (IPSYS) 
Conclusions: The camera handler can be useful in video-assisted neck surgery and other laparoscopic procedure (e.g. cholecystectomy) because of its versatility and stability, even in solo surgery procedures.

\section{7 \\ Risk Assessment in Minimally Invasive Surgery \\ A. Maura1, G. Galatà2, F. Rulli1,2 \\ ${ }^{1}$ Innovative Surgical Technologies (Medical Engineering School), '2Department of Surgery, University of Rome 'Tor Vergata'}

Introduction: Nowadays, although minimally invasive surgery (MIS) is a gold standard in many endoscopic procedures and technologically advanced instruments can be found on the market, this surgery is not riskless. Then it is important to analyze the risks in order to know and prevent possible complications about an instrument or a method.

Materials and Methods: We analyzed risks about technical equipment management, human errors, laparoscopic access and creation of working space, electrosurgery, ultrasound, and instruments' ergonomics.

Results: We observed that all the risks correlated to MIS can be avoided with the presence of a professional figure who has a strong knowledge about this kind of instrumentation and methods: the medical or bio-medical engineer, with the aid of specialized technicians.

Conclusions: There is the need for three approaches in order to minimize risk correlated to MIS: proper design of technological equipment, enhancement of training and crediting, utilization of checklists and protocols.

\section{8}

\section{A Dry Lab for Medical Engineers?}

\section{G. Galatà ${ }^{1}$, A. Maura ${ }^{2}$, F. Rulli $i^{1,2}$}

${ }^{1}$ Department of Surgery, ${ }^{2}$ Medical Engineering Course Innovative Surgical Technologies, University of Rome 'Tor Vergata'

Background: We describe a teaching and training method with objective evaluation to improve the medical engineering students' knowledge about Minimally Invasive Surgery (MIS) instrumentation and techniques through hands-on experience. Training has been scheduled during a three months course.

Methods: Course had theoretical and practical lessons. Handson exercises were performed on a simulator to assess whether specific training exercises were helpful for medical engineering students to familiarize with surgical devices and techniques. Eleven medical engineering students without surgical skills were trained to perform a sequence of standardized drills connected with the selected MIS techniques. The laparoscopic simulator consisted in a traditional pelvic trainer. The participants performed a sequence of tasks three times in order to improve their dexterity. The time required to perform each dexterity drill was recorded in seconds.
Results: All the trainees showed a steady improvement in skill acquisition on the laparoscopic simulator $(\mathrm{p}<0.05)$.

Conclusions: The course validity was demonstrated by measuring significant improvement in performance with increasing skillness. Dry lab experience for medical engineering students is useful for teaching and improving analysis and management of laparoscopic devices, allowing identification of problems with the aim of developing better devices.

\section{9 \\ Teaching and Training Model for Subfascial Endoscopic Perforating Veins Surgery: The Leg Trainer}

\author{
G. Galatà ${ }^{1}$ A. Maura², F. Rulli, ${ }^{1,2}$ \\ ${ }^{1}$ Department of Surgery, ${ }^{2}$ Innovative Surgical Technologies \\ (Medical Engineering School), University of Rome 'Tor \\ Vergata'
}

Introduction: SEPS (Subfascial Endoscopic Perforating Veins Surgery) is a simply reproducible and riskless minimally invasive video-assisted surgical procedure, useful in the treatment of Chronic Venous Insufficiency of the lower limbs. We suggest SEPS as a teaching model for a first approach to Minimally Invasive Surgery (MIS).

Materials and Methods: Our model consists of a learning period on the simulator, in order to assess the typology of tasks useful to improve technical skills in the video-assisted approach. The leg trainer consists of a hollow plastic model reproducing the left leg, filled with two concentric foam-rubber cylinders, miming fascia and muscles. These planes are intersected perpendicularly by isolated electrical cables, reproducing the leg's main vascular circulation.

Results: Our model allows performing several tasks, useful in the clinical practice: (a) reaching the subfascial leg space; (b) creating adequate operating space; (c) identifying insufficient vein(s); (d) coagulating and dividing them with the ultrasonic scalpel.

Conclusions: Our teaching and training model showed efficacy and reproducibility and is useful to teach the video-assisted approach. The procedure allows quantitative evaluation of the learning process and to assess improvements in individual technical skills.

\section{0}

\section{Cystic Dystrophy of the Duodenal Wall associated with Pancreatic Cystadenoma and Chronic Pancreatitis: A Rare Case Report}
L. Magno, G. Diamantis, A. Pascariello, M. Inzirillo,
A. Pastore, G. Galloro
Dip. di Chirurgia Generale, Geriatrica, Oncologica e Tecnologie avanzate - Area di endoscopia digestiva operativa. AUP Federico II

Introduction: Cystic dystrophy in heterotopic pancreas characterized by the development of true cysts from heterotopic pancreatic tissue localized in gastro-enteric wall. We describe the clinical and 
therapeutic features of a rare case of cystic dystrophy of the duodenal wall associated with a pancreatic cystoadenoma.

Case Report: A 44 years-old man was admitted in the gastroenterology unit of our University Hospital with epigastric recurrent pain and weight loss. A CT and a MR revealed an inhomogeneous thickening of the duodenal wall by multiple, coarse and septate cysts compressing common bile duct, Wirsung and duodenal lumen. To reach a differenziate exploration of pancreatic and duodenal areas, we performed a radial EUS showing:

thickening of the duodenal wall with multiple, intramural cysts arising from the third layer of the wall;

enlargement of pancreatic head, with a small cystic lesion with an own wall;

We diagnosed a cystic distrophy of duodenal wall associated with chronic pancreatitis and pancreatic cystic neoplasm. The patient underwent duodenopancreatectomy and was discharged after 14 days without postoperative complications.

Discussion: Cystic dystrophy in heterotopic pancreas is a rare condition with signs of upper digestive obstruction, sometimes with jaundice or signs of acute pancreatitis. CT and MR are useful in demonstrating the presence of cysts in a thickened duodenal wall but mainly EUS is able to reach a correct diagnosis. The choice of different therapeutic options is still debated: pancreaticoduodenectomy is proposed for symptomatic patients; however some authors have proposed medical approach using octreotide or endoscopic treatment for selected patients.

\section{1 \\ Primary Duodenal Epithelioid Angiosarcoma: Case Report}

G. Diamantis, L. Magno, A. Pascariello, M. Inzirillo,

A. Pastore, G. Galloro

Dip. di Chirurgia Generale, Geriatrica, Oncologica e Tecnologie avanzate - Area di endoscopia digestiva operativa. AUP Federico II

Introduction: Angiosarcomas are uncommon malignant tumor of vascular endothelium that represents less than $1 \%$ of all sarcomas. Angiosarcoma of gastrointestinal tract are exceptionally rare, they can arise in the esophagus, stomach, small intestine, appendix, colon and rectum. We here describe a rare case of primary duodenal epithelioid angiosarcoma.

Case Report: In July 2003, a 30-year-old white male, with a background history of anemia, was admitted in Gastroenterology Unit of our University Hospital presenting melena. An upper gastrointestinal endoscopy was urgently performed, showing a sessile polypoid lesion (about $1 \mathrm{~cm}$ diameter) in the third part of the duodenum, with irregular surface and a red-purple colour. Two days later a new UGE with polipectomy was performed. The histological examination revealed a malignant tumor with destructive pattern, ulcerating the surface epithelium. It was composed of epithelioid cells with round to oval nuclei and huge nucleoli. The cells were prevalently arranged in a solid pattern, featuring an epithelial growth. Immunohichemically the neoplastic cells were strongly positive for CD31 Factor VIII, and vimentin, and they were focally positive for keratin.

Discussion: Angiosarcoma of the gastrointestinal tract are exceptionally rare, with only a few case reports in the literature.
Gastrointestinal involvment can be primary or secondary to direct extension from intraabdominal 'angiosarcomatosis' or from a retroperitoneal disease, or metastatic. Primary Intestinal angiosarcoma usually presents with gastrointestinal bleeding and anemia. Endoscopy is often performed to identified the source of bleeding. The variant so-called epithelioid angiosarcoma, as in our case, that express also keratin, may make problematic the distinction from a carcinoma.

\section{2}

A New Endoscopic Treatment for Pancreatic Fistula after Distal Pancreatectomy: Case Report and Review of the Literature
A. Romano, M. Spaggiari, M. Masetti, R. Sassatelli,
F. Di Benedetto, N. De Ruvo, R. Montalti, G. Arzu,
G. Guerrini, R. Ballarin, M.G. De Blasiis, S. Di Sandro,
N. Smerieri, G. Rompianesi, G.E. Gerunda

Centro trapianti fegato e multiviscerale, Modena

Introduction: Despite surgical and medical improvements allowed a progressive decrease of morbidity and mortality linked to distal pancreatectomy over the last few decades, pancreatic fistula remains the single most common complication that influences the patient's clinical outcome and length of hospital stay. Several techniques have been proposed to prevent its development, but in the end minimally invasive procedures are highly desirable to shorten the fistula closure time with minimal risks for the patient. We performed endoscopic embolization of the duct by filling the leakage with glue to treat a relapsing pancreatic fistula in a patient undergoing distal panceatectomy for cancer of the pancreatic body.

Case Report: A 69 year-old woman had a postoperative pancreatic fistula after distal pancreatectomy for a pancreatic tail metastasis of colon cancer. As the pancreatic fistula did not heal by using conventional treatments, on POD 38 pancreatic fistula sealing was performed. A mixture of Glubran $2^{\circledR}$ (N-butyl-2-cyanoacrylate combined with methacryloxysulpholane by GEM, Viareggio, Italy) and iodinated lipid compound at a proportion of $0.5 \mathrm{ml}$ to $0.3 \mathrm{ml}$ was injected. The injection of the glue mixture was immediately followed by an injection of distilled sterile water, in order to fill the dead space volume and deliver the glue mixture into the fistula. Following cannulation of the main pancreatic duct with a guidewire confirmed a complete occlusion of the distal pancreatic duct. An associated pancreatic stent $(4-\mathrm{cm} 7 \mathrm{~F})$ was placed at the end of the procedure. As a result, the pancreatic fistula was sealed successfully and the patient was discharged on POD 45. An abdominal CT scan performed 6 months after showed a complete resolution.

Discussion: The management of a pancreatic fistula after distal pancreatectomy remains a big challenge. Despite the high rate of success $(70 \%)$, non-operative non-endoscopic treatment requires several weeks for spontaneous closure and a very long hospital stay. On the other hand, the surgical option in the early postoperative period has a success rate $>80 \%$ but increased mortality risk (between $23 \%$ and $63 \%$ ). In the last few years endoscopical treatment of post distal pancreatectomy fistula has had effective results in $75 \%$ of patients. In refractory cases, endoscopical pancreatic fistula sealing is performed with two different kinds of glue: fibrin glue and cyanoacrylate. The 
first is a physiological adhesive but is highly perishable when exposed to pancreatic juice, the second is a non-biological glue but can promote inflammatory reactions and most serious, embolic episodes. We present the first case of successful pancreatic fistula embolization with Glubran $2^{\circledR}$, a modified preparation of N-butyl-2-cyanoacrylate combined with methacryloxysulpholane, which has shown to have less cytotossicity and to induce a less severe inflammatory reaction. This procedure may be appropriate in some carefully selected patients if performed by expert endoscopists in a tertiary center. It has a few risks - compared to surgery; it does not require periodic treatment as fibrin glue does; and it reduces the hospital stay, with beneficial effects for the patient and cost savings.

\section{Phlebology}

\section{3 \\ Scleroembolization of the Left Spermatic Vein by Sandwich Technique}

\author{
D. Cecere, P. Valitutti \\ Chirurgia Vascolare, Agropoli
}

Introduction: Our relative experience to the endovascular treatment of male varicocele by scleroembolization of the left spermatic vein by sandwich technique.

Materials and Methods: 72 male patients aged between 17 and 42 years (average age 25.7 years) with left varicocele underwent clinical assessment, Doppler ultrasonography, testing of free and total serum testosterone, LH and FSH gonadotropins and spermiogram. In 58 cases selective phlebography of the left spermatic vein was performed with a right inguinal access. 52 patients underwent endovascular treatment of scleroembolization of the left spermatic vein. In 6 cases Polidocanol (Aetoxysclerol) 3\%, in 8 cases Gianturco coils and in 38 cases two Gianturco colis ( 1 below the inguinal ligament and 1 to III superior of the left spermatic vein) and Polidocanol (Aetoxysclerol) 3\% by sandwich technique were used.

Results: Immediate technical success was achieved in the 52 patients. At the 6 and 12 months follow-up they underwent Doppler ultrasonography and spermiogram. In 48/52 (92.3\%) patients showed complete resolution of varicocele whereas $4 / 52(7.7 \%)$ patients had only partial disergogement of the pampiniform plexus. In the 52 patients the spermiogram showed a significant increase in sperm concentration and the motility of the spermatozoa with negligible morphological changes. $3(5.8 \%)$ patients showed partial relapsing of the varicocele: $2(3.9 \%)$ patients underwent scleroembolization by only Polidocanol (Aetoxisclerol) 3\% and 1 (1.9\%) patients underwent embolization by only Gianturco coils.

Discussion: The endovascular treatment of the varicocele is reliable, effective, minimally invasive and economically viable technique that can be performed on a outpatient basis and above all to eliminate the venous spermatic reflux and improve seminal parameters.
54

\section{Treatment of Venous Ulcers: CHIVA vs. Compression Therapy}

F. Tovecci, R. Compagna, P. Sorrentino, K.A. Markaboui,

A. Bellino, B. Amato

Department of General Surgery, University 'Federico II' Naples, Chairman: Prof. G. Perisco

Introduction: In industrialized society the risk to develop a venous ulcer is very high and it continues to increase. Usually there are two kinds of treatment:

- Venous surgery

- Compression therapy

This study compares both techniques in patients with venous leg ulcers.

Methods: 60 patients with 68 venous leg ulcers have been selected and if they presented bilateral ulcers, each one was considered separately. We adopted some exclusion criteria:

1. Age $>70$ years

2. Incapacity to walk

3. Ulcer extension $<1.5 \mathrm{~cm}$ or $>10 \mathrm{~cm}$

4. Diabetes

5. Arterial and venous disease

Our patients were divided in two groups and we treated them separately.

Results: The most significant check-up was effectuated 2 years later and it showed that with CHIVA treatment healing was $99 \%$ and $95 \%$ with compression therapy. About relapses, the study shows a higher rate in the compression group.

Discussion: It's evident that the surgical treatment has much more success for leg ulceration than compression therapy.

55

\section{Association Between Recurrent Varicose Veins and Incompetent Perforating Veins: Our Experience}

\section{P. Sorrentino, D. De Vito, V. Piscitelli, R. Compagna, V. Di Maio, F. Tovecci, B. Amato \\ Department of General Surgery, University 'Federico II' Naples, Chairman: Prof. Giovanni Persico}

Introduction: The aim was to investigate the association between the presence of incompetent perforating veins and development of recurrent varicose veins.

Methods: A consecutive group of patients presenting with varicose veins were examined using colour duplex ultrasonography. Pathological perforating veins were defined as those exhibiting bidirectional flow and a diameter of $4 \mathrm{~mm}$ or greater at the fascia.

Results: Between September 2005 and October 2006, 224 patients were examined. Primary varicose veins were found in 246 legs (165 patients) and recurrent varicose veins in 84 legs (59 patients). In patients with primary varicose veins, 108 (44\%) had incompetent perforators compared to $53(63 \%)$ of those with recurrent varicose veins. Also, for recurrent varicose veins, the percentage of patients with any 
given number of incompetent perforators was higher than for primary varicose veins. Overall, there was a higher number of incompetent perforators in those with recurrent veins compared to primary veins and this difference was significant at $95 \%$ confidence interval.

Discussion: Patients with recurrent varicose veins have both a higher prevalence and a greater number of incompetent perforating veins than patients with primary varicose veins.

\section{6}

\section{Endovenous Laser Procedure in Saphenous Insufficiency: Our Experience}

L. Gazzabin, St. Mancini, R. Dell'Avanzato, G. Tommasino, V. Cassioli, A. Gazzabin, S. Mancini

Chir. Gen. $3^{\circ}-$ Centro Flebologico, Siena

Introduction: Our aim is to evaluate indications and results of the endovenous laser (EL) procedure in saphenous insufficiency.

Methods: In the last 6 years, we performed 2,021 surgical treatments of varicose veins. Among this, 184 were EL procedure. We included GSV with 6-12 $\mathrm{mm}$ diameter, without dilatations, rectilinear and no more deep than $1 \mathrm{~cm}$ from the skin. The procedure was performed in troncular or local anaesthesia by the introduction of a laser fiber throught a little incision or by the Seldinger technique. Duplex scan was necessary intraoperatively to evaluate the distance of the fiber from the $\mathrm{S}-\mathrm{F}$ junction (more than $2 \mathrm{~cm}$ ).

Results: All the 184 procedures were technically successful. No intraoperative complications occurs. Follow-up at 1 week and 1, 3, 6, 12 months showed: complete obliteration in $152(82.7 \%)$, incomplete obliteration in $12(6.5 \%)$ and recanalization in $20(10.8 \%)$. No postoperative pain, paresthesia, skin burn or deep vein thromboses were observed.

Discussion: Our experience demonstrates that in selected cases, EL procedure in saphenous insufficiency, can be safe and effective with a good compliance due to the less invasivity of the procedure. The preoperative study with Duplex scan of the S-F junction and of the saphenous vein is necessary to obtain good results with lower incidence of recanalizations and postoperative complications.

\section{7}

\section{The Sural Flap in the Treatment of Chronic Venous Ulcers and Complicated Wounds of the Lower Third of Leg, Hill and Malleoli Defect}

R. Dell'Avanzato, F. Bellezza, L. Gazzabin, St. Mancini,

G. Tommasino, V. Cassioli, G. Tancredi, S. Mancini

Chir. Gen. $3^{\circ}$ - Centro Flebologico, Siena

Introduction: The sural flap is considered, in literature, as one of the best treatment in chronic venous ulcers and complicated wounds of the lower third of leg. Particularly the complicated ulcers require the excision of the ulcer with surrounding lipodermatosclerotic skin, and replacement of the defect with healthy tissue.
Methods: The Authors analize the results of 22 (14 M; 8 F) consecutive patients, between 12 and 79 years-old; ten patients showed chronic venous ulcers of leg, 8 with outcomes of burn and 4 of electrocution. All patients were previously treated with surgical escarectomy and autologous dermal-epidermic graft. Most of lesions were localized in the inferior third of leg. Surgical procedure provides the rotation of the sural flap to cover the lesion.

Results: Postopertive course was uneventful in all cases but one with a transitory suffering of the flap, spontaneously resolved.

Discussion: The distally based superficial sural artery flap is an easily and versatile procedure, useful in reconstruction of lower third of leg, heel and malleoli defect. The surgical technique is safe and of short duration.

58

\section{Epidemiological Data and Ultrasonographical Findings in IVC}

St. Mancini, R. Dell'Avanzato, G. Tommasino, L. Gazzabin,

A. Comandi, V. Cassioli, S. Mancini

Chir. Gen. $3^{\circ}$ - Centro Flebologico, Siena

Introduction: Authors evaluated some epidemiological, anatomical, pathological and therapeutic data of chronic venous insufficiency (IVC).

Methods: We registered sex, age, familiarity, previous venous surgery, deep venous thrombosis and sclerotherapy, compression therapy. During the ECD exam we pointed out the great saphenous reflux, the presence of recurrence varicose veins and the anatomical level of saphenous-popliteal crossing (OSP) classified in: at $3 \mathrm{~cm}$ from the popliteal fold, inside 3 and $15 \mathrm{~cm}$, absence.

Results: The female prevalence was $76.5 \%$, and familiarity was $71.5 \%$. The ECD exam reported a great sapheous reflux in the $40.5 \%$ of the cases (familiarity $69.2 \%$ positive; $43.1 \%$ negative), while the external saphenous reflux only in $5.9 \%$. Finally DVT in the $4.1 \%$ (familiarity $11.2 \%$ positive; $15.6 \%$ negative), while $37.5 \%$ of patients performed a surgery, with a recurrence of $11 \%$. Compression therapy is utilized in $38.2 \%$ of the cases, of which $41.6 \%$ after surgery, $50-5$ of DVT and $53.2 \%$ with saphenous reflux. Sclerotherapy performed in $21.5 \%$ of patients. About anatomy: the OSP was in $57.2 \%$ at $3 \mathrm{~cm}$, in $33.8 \%$ at $3-15 \mathrm{~cm}$ and absence in $9 \%$ of the cases. Finally the saphenous reflux was junctional in $84.7 \%$, derived by collateral vein in $11 \%$ and by a perforating vein in $4.3 \%$ of the cases.

Discussion: Some data are very interesting and need to be discussed.

59

Elastic Stocking in Venous Ulcer Treatment

St. Mancini, R. Dell'Avanzato, G. Tommasino, L. Gazzabin,

A. Comandi, V. Cassioli, S. Mancini

Chir. Gen. $3^{\circ}-$ Centro Flebologico, Siena

Introduction: We report our experience with the use of elastic stocking in venous ulcers treatment. 
Methods: We perfomed two different multicentre study with stocking: the first with Tubulcus ${ }^{\circledR}$ was a research of phase IV, no comparative; the second with Ulcer- $\mathrm{X}^{\circledR}$ Kit was a randomised, double blind (major clinical evidence).

Results: The Tubulcus ${ }^{\circledR}$ study was completed by $66.6 \%$ of cases (33 on 57 patients) and one third dropped out in the first week of therapy for stocking unbearableness. We reported a complete healing in $56.1 \%$ of cases ( 32 patients), while in the other 4 a reduction almost $2 \mathrm{~cm}$ of diameter; in the last 2 cases we didn't verify any changes. No collateral effects were recovered. The Physician questionnaire showed a rather easy stocking putting on in $45.6 \%$ of cases, a little topical medication movement in $54.3 \%$, none in $22.8 \%$ and moderate in $21 \%$ of cases and at last an easy size choice in $59.6 \%$ of cases. Stocking was dressed 24 hours a day in only $49.1 \%$ of cases. The Ulcer- $X^{\circledR}$ casuistry is slighter with only 9 cases, but all healed in a maximum of 5 months for PTS ulcers and 4 months for varicose ulcers.

Discussion: Our limited experience with elastic stocking reported a lower time for healing, compared to normal bandage. It's of primary importance personalize the treatment, compressive included, basing on clinical, pathological and psychological aspects too.

\section{0}

\section{Laser Application in Venous Failure}

F. Tovecci, R. Compagna, A.K. Markaboui, P. Sorrentino, V. Di Maio, B. Amato

Department of General Surgery, University 'Federico II' Naples, Chairman: Prof. G. Perisco

Introduction: Untreated varicose veins have significant morbidity and potential mortality. Current therapeutic options include graduated compression stockings, sclerotherapy, ambulatory phlebectomy, surgical ligation and stripping. EVLT is a novel percutaneous technique for occlusion of the incompetent veins. Results of laser photocoagulation of vascular anomalies have been encouraging. Applying these concepts of laser-tissue interactions, we developed a new method of treatment for varicose veins of the lower extremities.

Methods: 25 patients with 27 varicose greater saphenous veins and 5 varicose smaller saphenous veins underwent endovenous laser photocoagulation (EVLT). Age ranged between 25 and 70 years. There were 15 females and 10 males. Saphenofemoral reflux has been documented by duplex ultrasounds. EVLP was performed using the $980 \mathrm{~nm}$ laser delivered with a $600 \mu$ fiber. Laser power was set at $8-10 \mathrm{~W}$. Patients were evaluated at 1 week and at $1,3,6,9,12,18$ and 24 months to determine efficacy and complications.

Results: The follow-up period from 1 to 9 months showed the common early complications of EVLT as local paraesthesia of the treated area, ecchymosis and superficial phlebitis and localized hematoma. The follow-up period from 9 to 24 months has an important role to diagnose possible recanalization or thigh's relapses. The final outcome showed no significant morbidity or mortality.

Discussion: EVLP is a simple effective treatment modality for varicose veins. This less invasive method can minimize the complications of conventional surgery and it makes the treatment a daysurgery treatment for the saphenous vein.

\section{1}

\section{Short Saphenous Vein Treated by EVLT}

F. Tovecci, P. Sorrentino, V. Piscitelli, R. Compagna, V. Di Maio, B. Amato

Department of General Surgery, University 'Federico II' Naples, Chairman: Prof. G. Perisco

Introduction: This study asses the safety and preliminary efficacy of endovenous laser treatment (EVLT) for occlusion of the incompetent short saphenous vein.

Methods: 20 patients with SSV incompetence have been selected by duplex ultrasounds studies and than they were treated with EVLT using a $980 \mathrm{~nm}$ diode laser. There were 15 females and 5 males with an age range between 30 and 70 years with a mean age of 50 years.

Results: All procedures were successful in the $70 \%$ of our patients. They did not show any recanalizations even at the final follow-up scanning; $93 \%$ of short saphenous veins remained closed. $2 \%$ of our patients had a local paraesthesia at the second follow-up examination ( 5 weeks). To avoid possible thrombosis or pulmonary emboli we treated all patients with fractionated heparin and Coumadin.

Discussion: EVLT results effective to eliminate short saphenous vein reflux and the complications seem to be negligible. We have found a low incidence of nerve injury instead the incidence of thrombotic events is higher than reported for the great saphenous vein.

\section{2}

\section{Complex Foot Trauma Treated by Vacuum- Assisted Closure}

V. Di Maio, R. Compagna, A.K. Markaboui, P. Sorrentino, F. Tovecci, A. Belli, B. Amato, R.G. Sottile

Department of General Surgery, University 'Federico II' Naples, Chairman: Prof. G. Perisco

Introduction: Several reports have documented the acceleration of granulation growth and decreased healing time of wounds with the use of the VAC device. Our goal was to evaluate our experience with the VAC device in the treatment of open traumatic foot wounds.

Method: The VAC device consisted of a sponge applied directly to foot's wounds, covered with impermeable transparent dressing, and attached to a low negative pressure system. The sponge was changed every 3 to 5 days under local sedation. We selected 24 patients with complex foot trauma divided in two groups on the base of treatment modality if it was continous or not. The kind of wounds treated, were widely fenestrated, infected and exposed fractures. This therapy has been applied in a range time from 4 to 8 weeks and any medical treatment has been required even if the group treated by the intermitted way needed an anti dolorific therapy.

Results: The continous way seems to be much more efficacy than the intermitted one infact in these patients. The use of the device has been shown to decrease the time to healing, to decrease length of hospitalizations, and to lower infection rates of complex wouds. Vacuum assisted closure was associated with rapid shrinkage, cleansing of the wound, excellent granulation, maintenance of a sterile environment and easy of use with changes possible at the bedsise. 
Discussion: The VAC device should be considered an effective alternative in treating complicated cases of foot trauma until a more definitive method can be used.

\section{General Surgery}

63

\section{Inguinodynia, Pubalgia, Inguinal Region Nerves Compression, Intramuscolar Hernia: A New Sindrome? A New Approach?}

\author{
S. Agrusti, D. Pettinari, M. Canziani, F. Frattini, M. Cavalli, \\ G. Campanelli \\ Policlinico Sant'Orsola Malpighi, Bologna
}

Introduction: 'Athletic pubalgia' affects people actively engaged in sports. It presents with inguinal pain exacerbated with phisical activity. The aim of this study is the critical dicussion of our surgical management in this painful syndrome.

Materials and Methods: From December 1997 to March 2007, 50 patients, with athletic pubalgia, males, with median age 27 years, were evaluated. All the patients underwent open inguinal repair modified Trabucco technique. The intraoperative findings was stiff insertion of medial pilaster of external ring and strong tension of the tendon of the rectus mussels; in this situation the ileoipogastric nerve are compressed. Moreover, superior edge esterne ring was very stiff, and determined compression of entire cord. The nerves intraptment was treated with partial section of the insertion of rectus mussels of the pubis, sculpture of a strip of lower portion of internal obl. and his positioning below the cord.

Results: After the median 4 years of follow-up, one patient showed chronic pain, but the others referrent disappearance initial symptoms. Overall log-term satisfaction was high and all returned to sports.

Dicussion: Persistant disabling groin pain on active sportsman is a frustrating diagnostic and management problem for both athlete and phisician. There is no consensus to support any particular surgical procedure, but an open approach, that resolve the nerve entraptment syndrome, relieve the pain and restores activity.

\section{4}

\section{Sutureless Fibrin Glue Incisional Hernia Repair}

\author{
S. Agrusti, D. Pettinari, M. Canziani, F. Frattini, M. Cavalli, \\ G. Campanelli
}

Policlinico Sant'Orsola Malpighi, Bologna

Introduction: Incisional hernia is a common surgical problem, frequently requiring prostethic mesh repair. The purpose of this study was to show the efficacy and tolerability of human fibrin glue $\left(\right.$ Tissucol ${ }^{\circledR}$ ) for non traumatic fixation of a polypropilene stiff mesh in open repair sutureless of incisional hernias.
Materials and Methods: A prospective study was performed including 40 patients ( 26 male, 14 female), with a mean age 56 years (range 38-75 years), having incisional hernias ( 25 epigastric, 10 subombelical, 5 total wall), who underwent open repair, between January 2002 to March 2007. The abdominal wall defect size range $5 \mathrm{~cm}$ to over $20 \mathrm{~cm}$. In all patients a polypropilene stiff mesh was used. The prosthesis was placed in retromuscolar or preperitoneal space, without fixation with stiches but using fibrin glue (Tissucol ${ }^{\circledR}$ ).

Results: In immediate follow-up, observed 4 cases of skin infection, 3 of hematoma, 2 of postoperatory pain and 2 of prolonged recovery. The average hospital stay was 4 days (range 3-8 days). During a mean long term follow-up, from 1 month to 5 years, there were 1 mesh infection, 1 recurrence and 1 case of cronic pain.

Discussion: The use of fibrin glue (Tissucol ${ }^{\circledR}$ ), improves the results of surgical repair of abdominal incisional hernias and decreases morbidity and hospital stay. The incisional hernia repair with sutureless fibrin glue, therefore appear to be safe and effective.

\section{5}

\section{Gastrointestinal Stromal Tumor (GIST) in the Emergency Setting - A Case Report}

\section{A. Amore ${ }^{2}$, M. Campione 1 , G. Biondi ${ }^{1}$, R. Chiaverini ${ }^{1}$,} A.Delli Carpini ${ }^{1}$, G. Di Sandro

${ }^{1}$ General Surgery SS. Rosario Hospital Venafro (IS), ${ }^{2}$ Corresponding Author

Objectives: GISTs are a heterogeneous group of mesenchymal neoplasms of the gastrointestinal tract in which routine histopathological examination fails to determine definitive evidence of differentiation. Recently, the improvements in immunohistochemistry have permitted outlining the histogenesis of these tumors. We report our experience of a GIST case diagnosed by chance.

Methods: The case was a 67-year-old male patient who had undergone surgical intervention for medium differentiated sigma adenocarcinoma ten years previously. The patient came to the S. Rosario di Venafro (Isernia) hospital with the diagnosis of strangled umbilical hernia: Following routine examinations the patient underwent surgery. An incision was made above the umbilical and opening the peritoneum revealed a hernia sac containing the omentum in which there were small friable formations which bled easily. This element raised the suspicions of the surgeon who proceded to extend the incision for complete exploration of the peritoneal cavity where there was a clearly visible speckled ansa of the small intestine. This was resectioned by a latero-lateral anastomosis. Surgery concluded with a stomach plastic to repair the defect of the umbilical hernia. Other small ulcerated formations were exported for histological examination.

Results: All the samples examined were sent to the pathologist. The histological response suggested a gastrointestinal stromal neoplasm with epithelioidal and fused cells, which had a high mitotic index and regressive necrobiotic areas.

Conclusions: Thanks to this emergency intervention it was possible to make an important diagnosis of gastroinsestinal stromal tumour (GIST). The patient after having undergone a total body CTscan was sent to the oncologist to undergo chemiotherapy. 
66

latrogenic Colonic Perforations: Early Endoscopic Repair by 'Triclip' - A Report of Two Cases

\author{
A. Trecca' , F. Gaj' ${ }^{2}$ F. Antonellis ${ }^{1}$ \\ ${ }^{1}$ Operative Units of Endoscopy and Gastroenterology \\ 'Fabio Di Giovambattsita' Rome, Italy; ${ }^{2}$ Department of \\ General Surgery, Second Surgical Clinic, University of 'La \\ Sapienza', Rome, Italy
}

The Authors herein present two cases of iatrogenic perforations occurred during both diagnostic and therapeutic colonoscopies. Due to the large parietal defects $(18 \times 8$ and $25 \times 10 \mathrm{~mm})$, prompt endoscopic repair was attempted using the TriClipTM device (Cook Endoscopy, Winston-Salem, MC, USA). This new device should guarantee a much higher grasping of the colonic mucosa due to its tripodic appearance. A complete closure was obtained in 8 and 35 minutes using three and four TriClipTM in the two cases. Both the patients did not need any further surgical treatment and were discharged uneventfully. Early endoscopic repair seems to be feasible even in case of large iatrogenic perforation with the new TriClipTM device.

67

\section{A 'Dangerous' Italian Dish: Spaghetti with Mussels}

\author{
A. Trecca' ${ }^{1}$ F. Gaj ${ }^{2}$, F. Antonellis ${ }^{1}$ \\ ${ }^{1}$ Operative Units of Endoscopy and Gastroenterology \\ 'Fabio Di Giovambattsita' Rome, Italy; ${ }^{2}$ Department of \\ General Surgery, Second Surgical Clinic, University of 'La \\ Sapienza', Rome, Italy
}

Spaghetti with mussels represents one of the most delicious italian recipes, except for our patient, a 43 y.o. man, who came to our attention just after eating this dish complaining of disphagia and sensation of foreign body in the throat. The exploration of the mouth carried out in the emergency department together with a laringoscopy had negative findings. Upper gastrointestinal endoscopy performed in our institution revealed the presence of a seafood localized in an unusual position: the left pyriform sinus. With gentle aspiration of the scope onto the seafood, we were able to extract this unusual foreign body whose diameter was $15 \mathrm{~mm}$. The patient recovered well and became free of symptoms.
68

Chronic Inguinal Pain after Hernia Repair: Mesh Weight and Resident Weight

\author{
A. Bondurri', G. Lenna ${ }^{1}$, V. Orvieni ${ }^{2}$, A. Spreafico ${ }^{2}$, \\ U.M. Monestiroli ${ }^{1}$ \\ ${ }^{1}$ General Surgery Department, IGEA Hospital, Milan, Italy; \\ ${ }^{2}$ Postgraduate School in General Surgery, University of \\ Milan, Italy
}

Introduction: To compare long-term pain after hernia repair with lightweight (LW) and heavyweight (HW) polypropylene mesh; to evaluate the effect of resident's intervention on recurrence and chronic pain.

Methods: In 2003, all patients with a primary inguinal hernia were randomized to repair using a heavy $(223 \mathrm{~g} / \mathrm{mq})$ or a light $(127 \mathrm{~g} / \mathrm{mq})$ mesh. The patients were operated by consultant or general surgery resident (PGY 4, 5 and 6) under supervision, and they were blinded to wich mesh they received. A telephonic interview was conducted after two years. Statistics performed with $\chi^{2}$ test.

Results: 132 patients were randomized, 76 to $\mathrm{HW}$ and 56 to LW mesh. 115 patients $(87 \%)$ were interviewed a median of 21 (range 15-34) months after hernia repair. There were no differences between the HW and LW mesh respect to recurrence (one in each group), chronic pain ( 38 vs. $51 \%$ ), pain severity (4 vs. 3.6 ), and groin discomfort ( 22 vs. $21 \%$ ). There were no differences between patients operated by resident and by consultant respect to recurrence ( 0 in both groups) and chronic pain (34 vs. 55\%).

Conclusion: Chronic inguinal pain persists in half patients two years after surgey. Use of LW or HW polypropylene mesh did not affect recurrence rate neither long-lasting pain neither inguinal discomfort. With adequate supervision, general surgery resident has recurrence and chronic pain rates similar to consultant.

\section{9 \\ New Perspectives in Follow-up after Gastric Surgery}

L. Graziosi, W. Bugiantella, E. Cavazzoni, P. Rossi, A. Donini

Department of General and Emergency Surgery, University of Perugia, S.M. della Misericordia Hospital, Perugia, Italy

Introduction: The benefit of a follow-up after radical surgery for gastric cancer is debated, but it is arising thanks to new chemotherapic and surgical strategies in the treatment of hepatic metastasis. Tumor Markers (TM) (CEA; CA 19.9; CA 72.4) and 18FDG-PET-CT are respectively cheap and heavily sensible systems and they could be suggested not only in the diagnosis but also for detection of early recurrences, as our prospective study shows.

Methods: 75 patients with gastric adenocarcinoma were preoperative studied with EGDS with biopsy, TM, USEGDS and PET-CT. After radical surgery they were followed firstly at 3 months and then every 6 months with US, EGDS and TM. PET-CT were performed every year or when recurrences were suspected. 
Result: TM preoperative positivity and sensibility were respectively: CEA $0 \%-0 \%$; CA 19.9, 5.8\%-7.5\%; CA 72.4, 20\%-20\%. Overall TM preoperative sensibility was $13 \%$. In 6 patients with peritoneal or hepatic recurrences sensibility was respectively CEA $16 \%$; CA 19.9, 33.3\%; CA72.4, 50\%; the overall TM sensibility was $66.6 \%$. TM positive and negative predictive value was respectively $71 \%$ and $91 \%$. We observed no false positive to CEA and CA 72.4 and no false negative to PET-CT.

Discussion: TM are useful in follow-up after gastric surgery and they may be suggested for clinical routine. CA 72.4 is the best marker of recurrences because of its best sensibility and specificity. Our study shows the importance of intensive follow-up after gastric surgery by integrating TM with imaging techniques so that patients could benefit of the new chemotherapic and surgical strategies.

\section{0}

\section{Early 'Buried Bumper Syndrome': Case Report}

M.T. Paganelli, F. Cantarella, W. Bugiantella, A. Pisciaroli, A. Donini

Department of General and Emergency Surgery, University of Perugia, S.M. della Misericordia Hospital, Perugia, Italy

Introduction: The buried bumper syndrome (BBS) is a rare, late and serious complication of percutaneous endoscopic gastrostomy (PEG) insertion for long-term enteral nutrition.

Methods: A 82-yo woman with Alzheimer's disease, unable to swallow safety, presented at our examination for complete occlusion of the tube gastrostomy making it impossible to feed her. She had undergone a PEG with 'pull technique' 20 days before. On clinical examination the stoma site was reddish and there was a leakage around the tube; the rigid bumper could be felt under the skin. We performed an emergency endoscopy and confirmed the BBS: the internal bumper ulcerated the gastric mucosa, migrated from the deeper gastric wall into the subcutaneous tissue. We easily removed the gastrostomy tube with a small incision of the abdominal skin and, preserving the existing fistula, we replaced a second tube by endoscopic method with preliminary gastropexy, using the same cutaneous site and over a guide wire. The patient was discharged on the first postoperative day without complications.

Result: The BBS is revealed by a gradual increase of resistance at feeding administration and during catheter cleaning caused by gastric mucosa overgrowth around the internal bumper. Bumper extra gastric localization is very dangerous because of the risk of peritonitis due to gastric juice or feeding solutions.

Discussion: We advise the approach with the endoscopic replacement of gastrostomy tube by gastropexy in patients with early BBS to prevent leakage of gastric juice into the peritoneum and fatal peritonitis or sepsis.
71

\section{Anisakiasis and Ileal Gist in Neurofibromatosis}

A. Chessa, G. Bongini, G. Ugolini, J. Martelucci,

A. Fontani, F. Civitelli, G. Tanzini

Department of Surgery, First Surgical Division, University of Siena

Introduction: Von Rechklinghausen neurofibromatosis can be associated to GIST presence.

Materials and Methods: A 38-old woman with ilean GIST and hepatic absence and a prior diagnosis of neurofibromatosis was admitted to our clinic from an infectious disease department. Abdominal ultrasound performed for rise after abdominal pain reported multiple hepatic abscesses with no presence of parasitic or bacterial infection. During specific antibiotic therapy, the patient undergoes an NMR which puts in evidence a $32 \times 27 \mathrm{~mm}$ solid lesion near to iliac right vessels and to an ileal loop and another $10 \mathrm{~mm}$ solid nodular lesion near to the posterior parietal peritoneum. A $7 \mathrm{~cm}$ ileal resection was performed and the specimen showed two primary GIST (1-CD117+CD34+ epitelioid cells 17/50 HPF mib1 20\% with nematoses in its context; 2 -spindle cells CD117+CD34+ 1/50 HPF mib1 $<5 \%$. High risk according to Fletcher). Nematoses belonged to Anisakis spp. Post operative period was good and the patient received a chemotherapy. A 18-month follow-up is disease free.

Conclusions: Enteric anisakiasis is a relatively rare disease that causes clinical symptoms similar to Crohn's disease, intestinal tuberculosis or neoplasmus characterized by granulomatosis. Nowadays it is not reported the association with ileal GIST. Thus, the presence of a GIST is typical in Von Rechklinhausen neurofibromatosis and anisakiasis retrieval in a resected bowl results to be occasional.

72

Synchronous and Metachronous Colorectal Carcinoma: Necessity of Pre-Operative and Post-Operative Colonoscopic Surveillance

R. Compagna, B. Amato, G. De Palma, V. Piscitelli, F. Tovecci, A. Bellino, P. Sorrentino, M. Persico

Department of General Surgery, University 'Federico II' Naples, Chairman: Prof. Giovanni Persico

Introduction: Synchronous and metachronous colorectal carcinoma have an incidence of $2-10 \%$. Synchronous carcinomas of the colon and rectum are of considerable clinical significance because of their frequency, the number of extratumors misssed and the difficulty of preoperative diagnosis. The purpose of the study was to evaluate, the survival of these patients underlying the importance of post-operative colonoscopic surveillannce to find any predicting factor that may influence the occurrence of metachronous cancer.

Patients and Methods: From 1999 to 2005560 patients with colorectal cancer were admitted to our Department. During follow-up examination 25 patients $(4.3 \%)$ were found to present with multiple primary colon cancers. $56 \%$ were synchronous and $44 \%$ metachronous tumors. Colonoscopy and double-contrast barium enema 
revealed the syncronous cancer in $66.6 \%$ of the cases. Typical colectomies, depending upon the segment of the location of the lesion were performed. The criteria of metachronous cancer were: occurence more than 12 months after curative surgery; with preoperative complete colonoscopy or one negative post-operative colonoscopic follow-up to rule out synchronous tumor; tumor arising from mucosa at a site other than anastomosis.

Results: The overall 5-years survival of the patients was $43.67 \%$. Mortality was $10 \%$ for multiple primaries. The overall 5 -years survival of the patients with multiple primaries tumors was $44.67 \%$.

Conclusion: Patients with colorectal cancer must be fully studied endoscopically. Lifelong regular post-operative colonoscopic surveillance is essential for colorectal cancer patients.

\section{3}

\section{A Comparative Study of Knowledge and Awareness of Colorectal, Cervical and Breast Cancers to Enhance Colorectal Cancer Screening}

\section{R. Compagna, B. Amato, A.K. Markabaoui, V. Di Maio, G. De Palma, P. Sorrentino, F. Tovecci, S. Masone \\ Department of General Surgery, University 'Federico II' Naples, Chairman: Prof. Giovanni Persico}

Aims: Despite high acceptance levels of mammography and cervical cancer screening by Italian women, adherence with colorectal cancer screening remains suboptimal. A better understanding of the relationship among cancer screening behaviours by women may provide insight into interventions to enhance colorectal cancer screening.

Methods: Six-eight persons to six different outpatient clinics in AOUP 'Federico II' di Napoli were given a study-specific questionnaire consisted of 12 statements on the incidence, presentation, detection, treatment and prognosis of colorectal, cervix and breast cancer.

Results: Sixty-eight individuals returned the questionnaire. Respondents had read more about breast and cervical cancer than about colorectal cancer. The proportion of correct answers for each statement on breast and cervical cancer was higher than for answers to corresponding items on colorectal cancer.

Conclusion: Women adherent to mammography and cervical cancer screening guidelines were significantly more likely to undergo colorectal cancer screening than those who were not adherent, although colorectal cancer acceptance in the adherent group was still suboptimal. Non-colorectal cancer screening visits that women already readily accept potentially represent a 'teachable moment' for the delivery of education and behaviour-related interventions aimed at reducing the burden of colorectal cancer. This highlights the importance of public education in colorectal cancer to allow an early diagnosis for the surgeon.
74

\section{Minimally Invasive Thyroidectomy}

M. Di Marino, G. Mastrobuoni, E.Coppola Bottazzi,

C. Cirillo, M. Cacciapuoti, S. Masone, M. Persico, G. Aprea

University of Naples 'Federico II'; School of Medicine, Department of General and Geriatric Surgery and Diagnostic and Operative Endoscopy. (Chief: Giovanni Persico)

Introduction: Minimal-access thyroid surgery (MATS) is a safe and feasible alternative to conventional thyroid surgery in selected cases. The advantage that this technique offers is an improved cosmetic result. The present study reviews our experience on thyroid surgery using a minimally invasive approach (MIT).

Methods: The study group comprise all patients undergoing MATS during the period May 2005-Oct 2006. Sixteen patients underwent MIT, 14 women and 2 men. Nine patients underwent hemithyroidectomy and twelwe total thyroidectomy; among the twelwe thyroidectomies five were adenomas, four multinodular goitre and three single nodule. Exclusion criteria included: thyroid cancer, conformation neck, previous neck irradiation or surgery, presence of significant thyroiditis, nodule size $>3 \mathrm{~cm}$ and thyroid volume $>25 \mathrm{ml}$. The operation was carried out through a $2-2.5 \mathrm{~cm}$ medial incision. All operation were performed by using Ligasure.

Results: Average operation time was $60 \pm 15$ minutes for lobectomy and $70 \pm 30$ minutes for total thiroidectomy. There was one case of transient hypoparathyroidism and two of postoperative haematomas. MIT was not converted to conventional thyroidectomy

Conclusion: MIT is a safe and feasible procedure. Advantage: brief learning curve, excellent cosmetic results and greater patient satisfaction. Disadvantage: limited indications and increases surgical time.

75

\section{LigaSure $^{\mathrm{TM}}$ : A Good Tool in Total Thyroidectomy}

G. Mastrobuoni, E.Coppola Bottazzi, M. Di Marino,

C. Cirillo, M. Cacciapuoti, S. Masone, M. Persico, G. Aprea

University of Naples 'Federico II'; School of Medicine, Department of General and Geriatric Surgery and Diagnostic and Operative Endoscopy. (Chief: Giovanni Persico)

Introduction: The electrothermal vessel sealing system (LigaSure ${ }^{\mathrm{TM}}$ ) facilitates operative haemostasis by fusing blood vessel walls to form a collagen seal. We report our experience with LigaSure $^{\mathrm{TM}}$ for thyroidectomy to test the hypothesis that it reduces operating time without increasing complications compared with standard vessel ligation.

Methods: We have studied two groups of patient: both groups were composed by 153 patients undergone Total Thyroidectomy; the first between Jan 2005-Jun 2007 by use of LigaSure ${ }^{\mathrm{TM}}, 98 \mathrm{~F}$ and $55 \mathrm{M}$, mean age 52 (14-72) years, and the second between Mar 2002Jan 2005, $91 \mathrm{~F}$ and $62 \mathrm{M}$, mean age 55 (16-79) years by using traditional ligature. Exclusion criteria were intrathoracic goiters and tumors.

Eur Surg Res 2008;40:61-183 
Results: Average operation time was 43 (35-60) and 74 (50-90) minutes respectively. Cases of transient hipocalcemia were 13 vs. 31 respectively. Monolateral recurrent laryngeal nerves lesions were 0 vs. 4; no bilateral lesions in both groups. In the Ligasure ${ }^{\mathrm{TM}}$ 's group there were three cases of reintervention for postoperative hemorrage, and four in the other group.

Conclusion: In our opinion LigaSure ${ }^{\mathrm{TM}}$ was as safe as standard vessel ligation for total thyroidectomy, with the benefit of reduced operating time and make easier and safe preserving of laryngeal nerves and parathyroid glands.

76

\section{Techniques of Oncoplastic Surgery}

\section{Cremone, R. Ruggiero, F. Procaccino, E. Procaccini}

Cattedra di Chirurgia Generale, Seconda Università di Napoli

Introduction: It doesn't make sense, for the breast tumour in the initial phase, to propose demolotive surgery when you can obtain similar results, in terms of free illness survival and recovery, in respect to mastectomy, also saving the breast in association with other therapies:Chemotherapy, Radiotherapy and Hormone therapy. So is born the 'minimal' surgery of breast cancer, to reduce the aesthetic damage, in respect to oncological radicalism. I believe, as more, that the life's quality of the patients depends, on the grand part, also for the containment of aesthetic damage.

Materials and Methods: Therefore, the necessity to improve the aesthetic results has been thought. The 'oncoplastic' surgery propose, after conservative treatment, new diverse technical surgery:

- different cutaneous incision based on neoplasia localization,

- gland mobilization,

- flap preparation, etc,

to optimize the aesthetic result. For the breast remodelling, attention must be paid above:

- the restoration of volume and form,

- the centralization of the areola,

and for the symmetry, if it is necessary

- a controlateral mastoplastic.

Results: The local relapse in the patients treated with QUART are percentually $6 \%$, versus $2 \%$ with mastectomy; a good-excellent aesthetic result in $84.8 \%$ with conservative surgery.

Conclusion: These simple but fundamental techniques, must build the cultural knowledge of the senological oncological surgeon, that must practice it himself or with the help of a plastic surgeon expertised in senological surgery.
77

Gastro Intestinal Stromal Tumours (GISTs): Our Experience and Histopathological, Histochemical and Biomolecular Concerns

D. D'Antonio, F. Pastena, N. Causa, G. Cioffi, A. lacono, A. Marvaso

General Surgery Department - A. Rizzoli Hospital - Lacco Ameno (NA) - Chief. Prof. A. Marvaso

Introduction: GISTs are the most common nonepithelial tumours of the gastrointestinal tract. Remarkable progress has been made during the last several years in their diagnosis and treatment. The molecular pathogenesis is linked to deregulated KIT tyrosinekinase activity, resulting in the successful application of imatinib, a systemic tyrosine-kinase inhibitor. A recent experience and a brief review of the literature are reported.

Methods: Between March 2005 and September 2006, four patients $(3 \mathrm{~F}, 1 \mathrm{M})$ were admitted in emergency for bowel obstruction. Mean age was 57.5 yrs (range 48-68). Two patients were lost at follow up. All cases required surgery. Intraoperative diagnosis was a 'high' bowel obstruction due to a jejuno-ileal mass, treated with a surgical open resection, followed by an end-to-end anastomosis. Morbidity and mortality were null. Histological diagnosis revealed a jejunal GIST with CD117- (KIT) and CD34- positivity.

Discussion: Our experience is at variance with other authors for localization and complained symptoms. GISTs mostly occur in stomach (50-55\%), then in small bowel (25-30\%), large bowel (10\%) and oesophagus ( $5 \%$ ). In $\sim 7 \%$ of cases GISTs can develop in omentum, mesentery and peritoneal cavity. In $\sim 30 \%$ of patients, GISTs are asymptomatic or with no specific signs (abdominal cramps, occult bleeding, haemorrhage). Less frequent are bowel obstruction, vomiting, perforation and dysphagia. About $50 \%$ of patients are admitted with metastatic disease, with mostly hepatic and peritoneal involvement. Tumour size and mitosis index are related with prognosis.

Conclusions: The management of GIST nowadays requires a multidisciplinary approach involving medical oncologists, diagnostic radiologists, gastroenterologists, surgeons and pathologists.

78

Stapled Haemorrhoidopexy: Evaluation of Histology, Intraoperative Morphology and Interindividual Anatomic Variability in MucoHaemorrhoidal Prolapse. Preliminary Results

D. D'Antonio', G. Luglio', D. Cerbone², L. Antinolfi',

${ }^{1}$ Department of General, Oncological and Videoassisted Surgery - Surgical Coloproctology Functional Area 'Federico II' University - Naples - Italy; ${ }^{2 ' S a n ~ F e l i c e ' ~ C l i n i c ~}$ - Pomigliano d'Arco, Naples, Italy; ${ }^{3}$ Department of Pathological Anatomy and Citopathology - 'Federico II' University - Naples - Italy

Introduction: Aim of the study was to correlate mucohaemorrhoidal prolapse morphology with histological features of excised 
tissue with stapled haemorrhoidopexy and evaluation of three-months results.

Methods: Eighteen patients with grade III and IV haemorrhoids were studied. Haemorrhoidopexy was performed with KYGZ-33.5 stapler. Intraoperative prolapsing tissue was evaluated: proctoscopic prolapse morphology, anal canal length, purse-string height. The excised ring was sent for histology. Early postoperative complication rate (haemorrhage, pain, sepsis) and short term results (preoperative symptoms improvement, incontinence, chronic pain, stool-clustering) were evaluated.

Results: In 2 patients no procidentia was present; in 2 patients there was haemorrhoidal prolapsing tissue; in 14 patients there was a protruding rectal tissue. Mean height of purse-string was $4.5 \mathrm{~cm}$. Histology revealed mucosa and submucosa in 4 cases; mucosa, submucosa and muscolaris propria in 9 cases and perirectal fat tissue in 5 cases. One patient complained postoperative pain. After three month, tenesmus was observed in one case, stool-clustering in two. Preoperative symptoms improved in 17 patients.

Discussion: There's a positive correlation between excised ring thickness and intraoperative concentric morphology of prolapse. Haemorrhoidal prolapsing tissue was observed in patients with a higher anal canal and a lower location of the purse string $(<4 \mathrm{~cm})$, with only mucosa and submucosa in the specimen. Rectal tissue protrusion in the supporting sleeve is associated with a thicker specimen (presence of muscolaris propria and/or perirectal fat tissue). The execution of a purse string more than $4 \mathrm{~cm}$ above the dentate line leads to better functional results at three months.

\section{9 \\ Surgical Resection of Colorectal Liver Metastases}

F. Di Benedetto, S. Di Sandro, N. De Ruvo, M. Masetti, R. Montalti, G. Arzu, A. Romano, G.P. Guerrini, R. Ballarin, M.G. De Blasiis, M. Spaggiari, G.E. Gerunda

Università di Modena e Reggio Emilia

Introduction: More than 9,40,000 cases of colorectal liver metastases occur annually worldwide and nearly 5,00,000 people will die from. Hepatic resection is the only potentially curative treatment, however $80 \%$ of patients with colorectal liver metastases remain unresectable at presentation. 5-years overall survival of resectable liver metastases resulted ranged between $20-50 \%$ versus $0 \%$ of unresectable liver metastases.

Methods: From October 2000 to August 2007, 125 patients underwent liver resection, in 4 cases associated with radiofrequency. Ablative technique, neo-adjuvant chemotherapy, and portal vein embolization were used to expand the rate of resectable liver metastases.

Results: 5-years overall survival resulted 33\% for patients underwent liver resection. 5-years disease free survival resulted $20 \%$. Lesion number and diameter, tumor grading, localization of the primitive tumor, and surgical margin infiltration resulted significant prognostic factors.

Discussion: Liver resection is able to offer long-term survival to patients affected by colorectal liver metastases. Several effective pre-operative treatment may expand the rate of resectability improving the long-term survival of these patients. Tumor progression before surgery is associated with a poor outcome, even after potentially curative hepatectomy.

80

\section{'Evaluation of Sensibility of Cytological Examination through Fine-Needle Aspiration in Thyroid's Lesions'}

\author{
C. Dobrinja, G. Trevisan, A. Romano, F. Zanconati, \\ G. Liguori
}

Department of General Surgery, Hospital of Cattinara, Università degli Studi di Trieste, Italy

Introduction: Fine-Needle Aspiration (FNA) is a reliable method in the initial assessment of thyroid nodules. Aim of this study is to evaluate causes of discordance between FNA's results and pathologic findings in surgical specimens.

Methods: 164 patients (pts), 134 women and 30 men with a mean age of 55 years were selected retrospectively in the Department of General Surgery, Division of Clinical Surgery of the University of Trieste, over a 7 years period (from January 2000 to June 2007). The preoperative cytological diagnosis was Inadequate $(\mathrm{C} 1)$ in 2 pts $(1.22 \%)$, Benign (C2) in 72 pts $(43.90 \%)$, Indeterminate (C3) in 45 (27.44\%), Suspicious for malignancy (C4) in 32 pts $(19.51 \%)$ and Malignancy (C5) in 13 (7.93\%). The results of FNA were compared with the surgical histological findings.

Results: The preoperative diagnosis resulted inadequate in $2 \mathrm{pts}$ $(1.22 \%)$ and discordant in 6 pts $(3.66 \%)$. The causes of the 6 discordant cases were: 1 case of C2 $(16.66 \%)$ was due to sampling error (false negative) and 2 cases of $\mathrm{C} 4(33.34 \%)$ and 1 case of $\mathrm{C} 5$ $(16.66 \%)$ were due to interpretation error (false positive). It is not always easy to correlate cytology and histology in those (2 cases of C3) indeterminate cases.

Discussion: Thyroid FNA is a simple and rapid diagnostic procedure with an acceptable accuracy rate that complements other investigations in the screening of patients for thyroid surgery.

81

Multidisciplinary Approach in the Treatment of Intrahepatic Lithiasis. Case Report

M.G. Esposito1, G. Conzo', V. laccarino², P. Venetucci², F. Stanzione1, U. Brancaccio ${ }^{1}$, A. Palazzo ${ }^{1}$, S. Celsi ${ }^{1}$, A. Livrea ${ }^{1}$

${ }^{1}$ IV Divisione di Chirurgia Generale e di Endocrinochirurgia Seconda Università degli Studi di Napoli, ${ }^{2}$ Radiologia

Cardiovascolare ed Interventistica-Università Federico II

Hepatholithiasis $(\mathrm{H})$ is defined as the occurrence of lithiasis in any intrahepatic bile duct proximal to the confluence. Primary $\mathrm{H}$ has an incidence of 4-47\% in Asiatic Countries while secondary $\mathrm{H}$ represents $<2 \%$ of biliary lithiasis in Western Countries. A surgical or a conservative treatment are mandatory to avoid evolutive complications such as hepatic atrophy, biliary cirrhosis, or cholangiocarcinoma. 
Case Report: In December 2006, a 67 ys male patient, with acute pancreatitis secondary to biliary lithiasis was observed. MR cholangiogram showed acute pancreatitis, choledocolithiasis without choledocous dilatation and massive secondary lithiasis associated to dilatation of 2, 3, 4 left bile ducts. After two months the pt refused left hepatectomy and underwent to transcutaneous hepatic cholangiogram, pneumatic divulsion of the papilla, clearance of bile duct and placement of external/internal biliary drainage. After a complete biliary tree cleaning by MTBE (metilterbutiletere) the patient underwent to laparoscopic cholecistectomy. MR cholangiography at three months showed a normal intrahepatic biliary tree with complete resolution of symptomatology.

Discussion: The treatment of $\mathrm{H}$ is still controversial. Transhepatic percutaneous approach is possible in case of unilateral disease without biliary stenosis and allows good medium-long term results. Hepatectomy is indicated in case of hepatic atrophy, biliary stenosis or suspected cholangiocarcinoma. A multidisciplinary approach - surgeon, radiologist, endoscopist - allows better results.

\section{2}

\section{Caudal Non-Functioning Pancreatic Endocrine Neoplasia (Pen) Presented as a Cystic Lesion. A Case Report}

F. Famà', A. Piquard², O. Saint-Marc', C. Linard'',
S. Mazzei', M.A. Gioffrè-Florio'
'Division of General Surgery, University of Messina,
Department of Human Pathology, Messina, Italy; ${ }^{2}$ Service de
Chirurgie digestive, Endocrinienne et Thoracique, Centre
Hospitalier Régional, Orléans, France; ${ }^{3}$ Service de Anesthésie
et Réanimation, Centre Hospitalier Régional, Orléans, France

Introduction: Pancreatic Endocrine Neoplasms (PENs) are a rare subset (1-2\%) of pancreatic tumors and may classified into functioning (60\%) and non-functioning (NF) (40\%) neoplasms. The most frequent radiological appearance is a solid mass. Occasionally cystic PEN has been reported in the literature.

Case Report: A 52-year-old woman was admitted for abdominal pain, vomiting and diarrhoea. A serum routine blood test revealed hyperleucocitosis with anicteric cholestasis. CEA and Ca19-9 were negative. Enhanced Computed Tomography (CT) showed a unilocular caudal pancreatic cyst (about $4 \mathrm{~cm}$ ). An open distal pancreatic resection was performed and the postoperative course was excellent. The patient was discharged on the 9th postoperative day. Histology exhibited a caudal cystic tumor $(39 \times 15 \times 9 \mathrm{~mm})$, confined to the pancreas and constituted by a cell population with few atypical nuclei. The mitotic rate was less than 2 per 10 High Power Fields and Ki-67 index $<2 \%$. Angioinvasion was detected. Immunohistochemical staining demonstrated positivity for chromogranin-A, synaptophysin and cytokeratin antibodies. A diagnosis of well-differentiated NF-PEN was made.

Discussion: PEN rarely manifest themselves as a cystic lesion and commonly cystic PENs are NF. The most frequent cystic lesions of the pancreas are pseudocysts (80-90\%). A preoperative differential diagnosis may be very difficult to make. The clinical behavior was evaluated according to the WHO classification. This neoplasm was considered to be uncertain in spite of the low mitotic activity and associated with the multiple endocrine neoplasia-1 (MEN-1) syndrome.
83

\section{Unusual Cause of Massive Lower Gastrointestinal Bleeding}
F. Famà', A. Piquard², O. Saint-Marc², C. Linard', S. Mazzei ${ }^{1}$, S.A. Villari ${ }^{1}$, A. Vindingi ${ }^{1}$, M.A. Gioffrè-Florio ${ }^{1}$
${ }^{1}$ Division of General Surgery, University of Messina, Department of Human Pathology, Messina, Italy; ${ }^{2}$ Service de Chirurgie Digestive, Endocrinienne et Thoracique, Centre Hospitalier Régional, Orléans, France; ${ }^{3}$ Service de Anesthésie et Réanimation, Centre Hospitalier Régional, Orléans, France

Introduction: Gastrointestinal Stromal Tumors (GISTs) are the most frequent mesenchymal neoplasms of the gut, especially occurring in the stomach $(60 \%)$ and less frequently in the small bowel $(20 \%)$. The clinical presentations of GIST is related to the tumoral size and those that come to clinical attention may represent an incidental finding. Symptoms are usually vague, although bleeding is frequent. An open or laparoscopic approach is reported in the literature.

Case Report: A 38-year-old man was admitted for bleeding and anaemia. The bleeding source was undetectable by endoscopy. Enhanced Computed Tomography (CT) showed a jejunal mass (about $22 \mathrm{~mm}$ ). The patient underwent open surgical excision. The postoperative course was uneventful and the patient was discharged on the 5 th post-operative day. Histology showed a submucosal tumor $(22 \times 20 \times 19 \mathrm{~mm})$, constituted by spindle-shaped cells mixed with fibro-hyaline stroma and negligible mitotic activity $(<5 / 50$ High Power Fields). The specimen was immunopositive to CD117, CD34 and $\alpha$-Smooth Muscular Actin antibodies. This tumor was classified, according to Fletcher, as being a low risk of aggressiveness.

Discussion: Upper gastrointestinal bleeding is a common clinical presentation of GISTs. Massive lower intestinal bleeding with tarry stools may be the first clinical occurrence. Endoscopic exams are the first-line examination. In some suspected cases of extramural tumors, ultrasound and Enhanced CT may be required, as suggested by this report. Magnetic Resonance Imaging, Angiography or Positron Emission Tomography may be performed for a more accurate characterization.

\section{4 \\ Enhanced Recovery (Fast-Track Surgery) in Colorectal Surgery: Two Years Experience}

F. Filidei, F. Scassa, M. Barlettai, R. Cantisani, M. Mazzillo, R. Mancini

U.O. Chirurgia Generale e d'Urgenza, Direttore

Prof. M. Seccia - Azienda Ospedaliera Universitaria Pisana

Introduction: Multimodal Fast Track Surgery (FT) combines optimal pain relief, regional anesthesia, early enteral nutrition and mobilization, to reduce surgical stress syndrome and improve perioperative outcome.

Methods: 52 patients were submitted to elective colorectal resection following a well defined protocol program based on meticulous open and /or laparoscopic technique, epidural analgesia, early
90

Eur Surg Res 2008;40:61-183 20th National Congress of the Italian Polyspecialist Society of Young Surgeons (IPSYS) 
oral feeding and mobilization, limited use and early removal of drains and catheters. Median age was 63 (35-78) with 27 females and 25 males. 29 patients underwent laparoscopic surgery (LS) and 23 open surgery (OS).

Results: Median LHS was 4 days for LS and 5 days for OS. Patients were able to feed with solid food on 1st day after surgery for LS and 2nd day for OS. Bowel movements occurred at 3rd p.o. day both in LS and OS patients. Overall morbidity (30 p.o. day) was $7.7 \%$; 3 readmissions $(5.7 \%)$ were due to anastomotic leakage, subocclusive episode and pleural effusion. Overall p.o. LHS with readmissions was 6.

Discussion: Postoperative hospital stay after traditional colorectal surgery ranges from 9 to 12 days and the morbidity rate is 15 to 20 per cent. Fast track surgery demonstrated to allow a significant improvement in p.o. recovery (pain, self managing, etc) and a better post operative course with a shorter hospital stay. No significant differences between LS and OS groups were noted.

85

\section{Enhanced Recovery after Surgery: Is it Safe and Feasible?}

C. Bagnato, F. Scassa, F. Filidei, M. Barlettai, E. Pressi,

R. Cantisani, C. Galatioto

U.O. Chirurgia Generale e d'Urgenza, Direttore

Prof. M. Seccia - Azienda Ospedaliera Universitaria Pisana

Introduction: Perioperative conventional management in colonic surgery is based on 'traditional' measures such as preoperative fasting, postoperative nasogastric decompression, nil-by- mouth until resumption of normal intestinal activity, late mobilization and removal of urinary catheter. Recent studies have emphasized that an enhanced rate of recovery can be achieved with a different post operative management.

Methods: 52 patients (27 F/25 M; median age 63) underwent major colorectal surgery, both open or laparoscopic, with a protocol based on optimal pain control with continuous epidural analgesia, avoidance of routine use of naso-gastric tube, oral intake before operation and from postoperative day one, early mobilization and removal of drains and urinary catheter.

Results: Mean hospital stay was 5.7. Return to normal gastrointestinal function was obtained on median day 3; urinary catheter was removed on day 1 and for 3 patients only repositioning was needed. NG tube insertion has been done in $6(11 \%)$ patients for PONV. The majority of patients $(80 \%)$ were mobilized on the same day of the operation. Three patients $(5.7 \%)$ were readmitted in hospital with an overall morbidity of $7.7 \%$.

Discussion: An enhanced-recovery protocol leads to an early resumption of normal functions without significant increase of readmission or morbidity rates in comparison with 'traditional' care.

\section{6}

\section{Grynfelt's Hernia in Day Surgery}

F. Gilio, P. Sperlongano, F.A. Malinconico, G. Moccia, F. Foroni, C. Cognetti, G. Iside, A. Gubitosi, M. Agresti

$X$ Divisione di Chirurgia Generale e Fisiopatologia

Oncologica - AUP - SUN, Napoli

Introduction: Lumbar hernias are very uncommon conditions. Less than 300 cases have been reported in literature they represent the $2 \%$ of the abdominal wall hernias. The lumbar defects are rarely $(10 \%)$.

Methods: We describe here one case of a large Grynfelt's hernia in a 67 years old man suffering for a rigth inguinal hernia too. The diagnosis was riched by physical examination supported by US scan and CT scan. The treatment consisted in the contestual polypropilene mesh repair of both defects under local anesthesia, step by step, reaching the global volume of $250 \mathrm{ml}$ of mepivacaine $0.25 \%$.

Results: The operation starts with a lumbar incision parallel to the 12th rib; the mass was reduced after the divarication of the upper located muscles. The prosthetic repair consisted in the allocation of a two layers polipropylene mesh above and beyond the defect borders, under the gran dorsalis muscle. The mesh was sutured to the muscle-aponeurotic tissue surrounding the defect borders and further on it was fixed by fibrin sealant to the neighbouring muscle's belly.

Discussion: Prosthetic repair has to be performed considering of the dynamic modifications of the wall structure. The mesh dimentions have to be adequate to the defect's width but also to the dimentions of all the endoabdominal strength enforcing area under efforts. We think, indeed, that this affection can be treated in one day surgery in the obvious discharging criteria procedure respect.

\section{7}

\section{Unusual Metastasis of Left Colon Cancer: Considerations on Two Cases}

\section{Cognetti, A. Gubitosi, G. Moccia, F.A. Malinconico, D. Parmeggiani, F. Gilio, F. Foroni, G. Iside, P. Sperlongano, M. Agresti \\ $X$ Divisione di Chirurgia Generale e Fisiopatologia Oncologica - AUP - SUN, Napoli}

Introduction: Left colon cancer metasyasis usually concerns the abdominal lymph nodes, liver, and lungs; other localizations are very rare occurrences. Uncommon metastasis sites have been reported in the literature on the tibialis anterior muscle, maxillary sinus, thyroid colloid nodule, scrotum, penis, testis, uterine cervix, knee synovial and skin.

Methods: We report two cases of unusual colon cancer metastasis. A 60 year-old male came to our clinic with a diagnosis of peritoneal mass. He had previously undergone a left hemicolectomy in another clinic. MRI-scan showed an intraperitoneal mass (diameter $5 \mathrm{~cm}$ ) below the left kidney. Others procedures showed no alterations on the other tracts. Another case was a 49-year old male with an abdominal lymphnode metastasis from a neoplasm of the left colon, 
treated, 3 years earlier with resection and colostomy. We found a large mass in the left axilla area.

Results: The first patient underwent laparotomy: none was found however, on the left parietocolic exploration; instead the mass of $9 \mathrm{~cm}$ was found in the retroperitoneal space. The mass was excised. After a regular post-operative course the patient was treated by radiotherapy. The second patient underwent the excision of the axillary mass and exploratory laparotomy that revealed another mass between the liver and pancreas. The patient was sent to radiology for abdominal and axillary radiotherapy.

Discussion: Retroperitoneal metastasis are uncommon $0.1 \%$, some authors underline the role of mesorectum excision. For the second our hypothesis is that from the colostomy area trough the lymphatic vessels the neoplastic cells reached the chest lymph nodes and then the axillary ones.

\section{8}

\section{Mesenteric Panniculitis: Case Report}

F. Foroni, P. Sperlongano, F.A. Malinconico, G. Moccia, F. Gilio, C. Cognetti, G. Iside, A. Gubitosi, M. Agresti

$X$ Divisione di Chirurgia Generale e Fisiopatologia

Oncologica - AUP - SUN, Napoli

Introduction: Mesenteric panniculitis is an extremely rare inflammatory condition whose etiology is still unknown and it is not easy to detect, confounding the nosology by the variety of its diagnostic-clinical features that often characterize it, like acute appendicitis, IBD, neoplasms, metastasis, ovarian torsion, Weber-Christian disease, bowel tbc, etc.

Methods: In this Case Report we describe one case of mesenteric panniculitis in a 33 year-old male that presented to our clinic with a sub-obstructive syndrome and an abdominal mass in the right iliac fossa.

Results: Diagnosis by physical examination was not easy; USand CT scan did not reveal any specific signs; final diagnosis was enriched by postoperative histopathological analyses.

Discussion: The etiology of this affection is still debatable; it seems that an autoimmune reaction can be involved, within a vasculopathic framework or linked to surgical operations, but pharmacologic or infectious factors cannot be neglected in some cases. The mesentery undergoes a massive fibrotization that is the major cause of symptomatology. Knowledge of this rare syndrome should avoid inappropriate therapy and could help the physician to use clinical, radiological, and surgical sources to obtain the diagnosis. This report should contribute toward understanding a disease whose diagnostic and therapeutic features are still disputed.
89

\section{Prosthesic Treatment of the Inguinal Hernia} in the Cirrhotic Patient

\author{
A. Gubitosi, G. Moccia, F.A. Malinconico, F. Foroni, G. Iside, \\ F. Gilio, M. Agresti
}

Seconda Università di Napoli - X Divisione di Chirurgia

Generale - (Dir. Prof. M. Agresti)

Introduction: The surgical approach to the cirrhotic patient, carrier of inguinal hernia, constitutes material of extreme scientificdoctrinal interest for the inevitable involvement of several specialistic (internistic, hepatologic, anestesiologic, as well as surgical) that, in different measure, contribute to define the prospects of treatment and recovery. The multidisciplining of such problems isn't able, up to-day, to give univocal information regarding the target, the operating time and the use of a specific and reliable surgical methodology for the particular tipology of the patient. However, a careful estimate of clinical-biochemistry estimate allows, in the preliminary research diagnostics, the singhing out of the hemato-chemical alterations typical of the pathology of basis, that suitably balanced, together with further prophylactic protections, allow to prepare adequately the patient for the operation, even in Day Surgery, with such prospects of recovery to level him with any other patient liable to operation.

Materials and Methods: They come taken in consideration and inserted in software epidemiologist - statistical (Epi Info 3.3.2), 49 variable relative ones to: age; sex; associated metabolic pathologies; associated erniarie pathologies; all variable possible the relative ones to the erniaria tipologia (mono/bil - center - prim./rec. - type complicata/non - class. magnitudo - class. Gilbert); the tipologia of the cirrosi and its eventual complicanze, with relative its two score you to the classification of Child and the preoperative and postoperative MELD; the therapy medical - surgical preoperative and post-operative; the anesthesia; the participation; the type of prosthesis; the piastriniche pre-intra and post-operative infusions; the application or less than glue than human fibrin; the post-operative complicanze intra and; the tipologia of shelter and finally the follow up. The end point primary it is represented from the verification of turns out obtained in connection with the specific protocol of adopted treatment (re-balance to you base parameters, antibioticoprofilassi in the short term; not opening of the possible bag when; possible local anesthesia when; use of human fibrin glue always; infusion of necessary and possible concentrated piastrine when; jam with bende adhesive elastic post-operative). Numerous variable the relative ones to pathology of base and the erniario defect will come correlated to the outcomes that they will be obtained in order to characterize, eventually, end points secondary relati you to the disease stage and to it turns out to you you achieve yourself, in the optical of the attainment of the possibility of application of protective protocols specific us in reason of the state of the patient in connection with base pathology. Are analyze in the study all to you the cirrotici patients (48) joints to our observation for erniaria pathology are single that multiple in last 4 years (2002-2006). The duration, the type of shelter and the follow up conclude the collection of the data.

Results and Conclusions: In our series of 48 patients we have not had intraoperatorie complicanze. The only post-operative complicanza has been represented from a inguino-scrotale small sieromatoma that it has not demanded reintervento of water-drainage. Follow-up not the extension today some recidiva neither complicanze in the long 
term. We think therefore, seppur in the esiguità of the casuistry, to continue in the application of our simple observed protocol having until today, turns out to you absolutely encouraging. It goes held present that the methodology can, in a considerable number of cases, being applied in Day Surgery, where are the optimal conditions from the internistico point of view, with all the benefits legacies to the same clinician-charitable model that, in the case of cirrotici patients, often in ambulatoriale treatment near the clinics for infectious diseases, assumes one clinician-associate-administrative valence of absolute relief.

\section{0}

\section{A Simple Indicator of the Correct Nasogastric Suction Tube Placement in Children and Adults}

\author{
A. Maura1 , G. Galatà ${ }^{2}$, M. Villa², F. Rulli, 2 \\ ${ }^{1}$ Innovative Surgical Technologies (Engineering School), \\ 'Department of Surgery, University of Rome 'Tor Vergata', Italy
}

Introduction: The Aim of this preliminary study was to determine the validity of a flexible optic fibres cable inserted into a naso-gastric tube in assessing a correct gastric placement in children and adults.

Materials and Methods: The study enrolled sixteen patients undergoing surgical procedures (eight beta-thalassemic children and eight patients submitted respectively to open splenectomy and laparoscopic cholecistectomy). A flexible cable of $1.3 \mathrm{~mm}$ diameter, connected with a cold light source was inserted in a common naso-gastric tube (NT) and advanced up to the epigastric area that was fully trans-illuminated. Aspiration with a syringe was practiced to emptying the stomach. The NT's size varied from 8 to 19 French $(2.7$ to $6.3 \mathrm{~mm})$.

Results: All of the patients had epigastric area illumination and the liquid obtained from the NT aspiration was of clear yellow colour. Moreover, in $100 \%$ of the patients, the correct position of NT was confirmed at the time of operation and no post-insertional complications were recorded.

Conclusions: The preoperative insertion of a flexible optic fibres cable into a NT may be useful in assessing the correct progression of the device through the oesophagus, and its placement inside the stomach. This instrument also allows preoperative stomach emptying. We believe that this finding deserves particular consideration as it represents a useful, safe, and cost-effective tool for surgeons. This particular device could be useful also in critically ill patients requiring gastric or enteral feeding, in which a frequent control of the tube placement is needed.

91

\section{Umbilical Endometriosis Associated with Umbilical Hernia. Management of a Rare Occurrence}

F. lovino, E. Irlandese, S. Gilli, R. Ruggiero, R. Setaro, F. Lo Schiavo

Chirurgia Generale, Policlinico SUN, Napoli

We report a rare case of primitive umbilical endometriosis associated with umbilical hernia, which required wide umbilical resection and immediate reconstruction performed according to a technique recently described. A wide resection of the peritoneal sac was performed because of the possible presence of endometrial tissue inside. Hernia was repaired according to Mayo's technique. Umbilical reconstruction was performed using two semicircular skin flaps shaped into in an ellipse preoperatively designed on the skin with a vertical orientation. The surgical approach described in this report allowed easy hernia repair and umbilical reconstruction. No prosthesis was used because of the small size of the hernia and lack of in literature data on prosthesis use in endometriosis. The aesthetic result was considered satisfactory after six months from the operation because of absence of hypertrophic scar and the anatomical aspect of the new umbilicus. No recurrence was observed within this time frame.

\section{2}

\section{Factors Affecting the Risk of Small Bowel Anastomotic Failure following Emergency Surgery}

A. Gurrado, G. Lissidini, D. Lardo, E. Poli, L. Nacchiero,

L. Ruggiero, M. Signorile, M.P. Valentini, M. Testini

Department of Applications in Surgery of Innovative Technologies, Section of General and Thoracic Surgery, University Medical School of Bari, Italy

Introduction: The leakage of anastomosis remains the most serious postoperative complication in gastrointestinal surgery. Anastomosing two ends of normal small bowel (SB) in a healthy patient is successful and uneventful in $99 \%$ of cases. The presence of comorboid conditions as infection, obstruction, bowel gangrene and malnutrition, impairs the normal healing of an anastomosis to some degree. Incidence of SB fistulae resulting from emergency surgery shows a mortality rate from 25 to $50 \%$. Aims of this study are to analyse the outcome of a series of patients who underwent SB resection and anastomosis in emergency, and to stress the factors affecting the risk of anastomotic failure.

Methods: Between 1997-2006, 114 small bowel anastomoses (SBA) were constructed in 99 patients (59 F, $40 \mathrm{M}$, mean age: 53, range: 17-89). All operations were performed in emergency and all patients were operated on by the same surgeon; intestinal volvulus, mesenteric ischemia, strangulated hernia and complicated Crohn Disease were the most common etiology. 83 (72.8\%) anastomoses were manually constructed versus $31(27.2 \%)$ stapled anastomoses.

Results: In 14/104 SBA (13.5\%) we observed the appearance of leakage: $10 / 114(8.8 \%)$ were complicated by an entero-cutaneous fistula; in 4 patients $(3.5 \%)$ re-surgery was necessary due to peritonitis. Peritonitis, malnutrition, and COPD were factors significantly affect SBA. There was no peri-operative mortality.

Discussion: This study shows that hypoalbuminemia, peritonitis, and chronic obstructive pulmonary disease are associated with higher incidence of anastomotic failure. On the contrary, age, use of corticosteroids, etiology, associated diseases and type of suture show no statistical significance. 
93

\section{Inguinal Hernia Repair by Surgical Trainees vs. Experienced Surgeon: A Retrospective Review}

\section{Lardo, G. Lissidini, A. Gurrado, E. Poli, L. Nacchiero, \\ L. Ruggiero, M.P. Valentini, R. Marzaioli, M. Testini}

Department of Applications in Surgery of Innovative Technologies, Section of General and Thoracic Surgery, University Medical School of Bari, Italy

Introduction: Aim of this study is to assess the outcome of primary inguinal hernia repaired by surgical trainees.

Methods: A retrospective review was conducted on 465 patients (23 $\mathrm{F}$ and $442 \mathrm{M}$, mean age 45.9 years, range $16-88 \mathrm{yr}$ ) electively operated on by the same experienced surgeon for primary inguinal hernia between 1997-2006.The operation consisted in a tension-free plug and mesh repair. 123 operations were performed by an experienced surgeon (group A); 144 (group B) and 198 (group C) were performed by residents with an experience level respectively of more and less than ten cases assisted by the same experienced surgeon of group A.

Results: There were no postoperative death in each group, and the mean operative time was $42 \mathrm{~min}$ in group A, 63 in group B, and $82 \mathrm{~min}$ in group $\mathrm{C}(\mathrm{p}=0.0001)$. The immediate morbidity rate was $4.1 \%$ in group A vs. $4.2 \%$ in group B vs. $4.0 \%$ in group C ( $p=N S)$. No significant differences were found between the groups in length hospital stay and mean time to return to work. Recurrence rate was $2.0 \%$ in group B $(2.0 \%), 0.8 \%$ in $\mathrm{A}$, and $1.0 \%$ in $\mathrm{C}$.

Discussion: The familiarity with groin anatomy and the technical skill gained in performing this operation are mandatory in the training of the young surgeon. Trainees easily acquire this skill if a specific protocol in their learning curve is performed by experienced surgeon. The only significant differences were observed as being relevant to the mean operative time.

94

\section{The Use of Percutaneous Catheter Drainage of Massive Abdominal Fluid Collections (Ct- and Us-Guided) to Preserve from Re-Surgery}

\author{
W. Lavermicocca, G. Lissidini, P. Pedote, A. Gurrado, \\ D. Lardo, E. Poli, L. Nacchiero, R. Marzaioli, M. Testini \\ Department of Applications in Surgery of Innovative \\ Technologies, Section of General and Thoracic Surgery, \\ University Medical School of Bari, Italy
}

Introduction: From the initial experiences in the late ' 70 s, percutaneous drainage has become the elective treatment for most of abdominal fluid collections. It is a safe and mini-invasive TC- and US-guided procedure, with low morbidity and almost no mortality. Thanks to the portability of US devices it may be performed bedside, which is a notable feature in the treatment of patients in severe clinical conditions.

Methods: The Authors report their experience in percutaneous drainage of abdominal abscesses and fluid collections as a primitive disease or following elective and emergency surgery. Five cases ( $3 \mathrm{M}$ and $2 \mathrm{~F}$; mean age $60.8 \mathrm{yrs}$; range: $27-84 \mathrm{yrs}$ ) in which the US- or CTguided placement of a pigtail catheter successfully succeeded in draining abdominal abscesses, were reported: 1 post-traumatic in a gunshot wounded patient; 1 postoperative in operated for left colon cancer; 1 with liver abscess in patient with high cardiovascular risk; 1 following Hartmann procedure in perforated diverticula of the sigmoid colon in immunodepressed patient; 1 with liver abscess in patient with progressive respiratory failure; thus avoiding surgery in patients with high operative risk.

Results: Percutaneous drainage proved to be a very useful tool in the hands of the surgeon for nonoperative (mini-invasive) treatment of abdominal abscesses and fluid collections, especially in critical patients even in trauma ones.

Discussion: Percutaneous drainage is a feasible, sometimes resolutive approach for the treatment of abdominal abscesses and fluid collections, especially in patients with high operative risk. It may be performed bedside by the surgeon himself under US-guide.

\section{5 \\ Technological Innovations in Surgery \\ L. Leone, M. Catarci, S. Drago, A. Di Cintio, A. Di \\ Gregorio, A. Torcasio, G.B. Grassi \\ Department of General and Oncological Surgery, San Filippo Neri Hospital, Rome}

The technology has allowed the surgery to make steps from giant in last two centuries. The surgical participations, made today in the moderns operating rooms, are the fruit of scientific evolution allowing the man to be able to push succeeding to enter today in the concept of surgical technology. Such concept at the moment in continuous evolution thanks to instruction programs and worldwide diffusion. One time the surgical operation was considered a challenge that man offered to the nature; the errors, the deaths, and multiple varying the surgical ones have allowed the surgeon, to improve themselves to enter in the field of the modern surgery. This science today is the base of our well-being, medium advance age and to the improvement of the quality of life in our society. The diagnosis computerize- systems, laparoscopic surgery, interventional radiology allows the surgeon to plan operating action reducing the surprise recognizing that are emerging in the field, far away from spectacular suggestions, without to exceed the limit of professional ethics.

\section{6}

\section{Congenital Diaphragmatic Hernia in Adult}

A.M. Lucisano, D.P. Pafundi, F. Pata, R. Calabria, R. Sacco

General Surgery, 'Magna Graecia' University, Catanzaro, Italy

Introduction: Diaphragmatic hernia is a protrusion of one or more abdominal bowels in chest trough a gap in this muscle. We can distinguish congenital, traumatic and secondary kinds; they can be 
asymptomatic or begin with grave clinical pictures in infants. Diagnosis is radiological. Therapy is always surgical in symptomatic forms.

Methods: We report a case of a female, 45 years-old patient with abdominal pain and dyspepsia, and negative physical examination. The standard laboratory data showed increased levels of RBC, WBC, platelets and D-dimerous, and decreased blood calcium and potassium. Chest and abdomen x-ray showed abnormal colic gas distension and presence of colic loop between diaphragm and liver, confirmed by enema $\mathrm{X}$-ray and CT scan. The urgent surgical procedure was right and left part of transverse colectomy (for volvulus) and the stitching of diaphragmatic gap.

Results: After surgery, symptomatology relapsed.

Discussion: Congenital diaphragmatic hernias are dued to embryonal defects, that consist in persistent gaps or failed fusion. We can distinguish anterior and posterolateral forms, as in this case. Clinically, if in child the beginning is acute, in the adult diagnosis is often accidental and is carried out by chest/abdomen and enema $\mathrm{x}$-ray, and CT scan or MNR. In symptomatic forms, surgical approach is by laparotomy, and it consists in replacing of herniated bowels and closing the muscular gap by prosthesis or simple suture of the defect.

\section{7}

\section{TRIMprob in Diagnosis of Bladder Cancer: Preliminary Results}

F. Pata, A.M. Lucisano, D.P. Pafundi, R. Sacco

General Surgery, 'Magna Graecia' University, Catanzaro, Italy

Introduction: TRIMprob (Tissue Resonance Interaction Method Probe)is a new diagnostic tool that exploits the different electromagnetic properties being between normal and pathological tissues. Aim of this study is to evaluate TRIMprob efficacy in diagnosis of bladder cancer, in patients with clinical suspect of neoplasy or during follow-up.

Methods: Between January 2005 and March 2006, 140 patients have been studied with TRIMprob; they have had an indication for cystoscopy for haematuria ( 90 pts) or during the follow-up for superficial bladder cancer T1 (50 pts underwent to TURV). The examination has been carried out in blind, from the same team operator, and repeated at different distances $(120$ and $150 \mathrm{~cm})$. We have been considered as positive a signal decrease below 50 U.A. in the red band (465 MHz). TRIMprob responses have been compared with cystoscopy and biopsy results.

Results: At TRIMprob examination, 56 patients have resulated as positive and 84 negative for neoplasy. In the group of 'positive' patients, 46 were true positive, and 10 were false positive. Among the 84 patients negative at examination, the true negative were 80 , and the false negative were 4 . Sensibility was $92 \%$, specificity $88.8 \%$.

Discussion: TRIMprob is a portable, non invasive, cheap and repeatable system. If these results will be confirm in wider populations, TRIMprob could be a new diagnostic tool for screening and follow-up of bladder cancer.
98

\section{Comparison Between Minimally Invasive Video Assisted Parathyroidectomy (MIVAP) and Minimal Access Parathyroidectomy in Terms of Surgical Education and Training}

\author{
G. Materazzi, P. Berti, G. Docimo, G. Frustaci, A. Fosso, \\ G. Donatini, P. Miccoli \\ Department of Surgery, University of Pisa
}

Recently, radioguided parathyroidectomy (RP), minimal access parathyroidectomy (MIP) and videoassisted parathyroidectomy (MIVAP) were proposed for the treatment of primary hyperparathyroidism. Both MIP and RP consist of a targeted exploration through a small incision. Traditional instruments are used and direct vision is utilized. MIVAP is performed through a $1.5 \mathrm{~cm}$ central incision. The operation is conducted under endoscopic vision. Since 1997, 618 patients underwent MIVAP. There were 514 females and 104 males. Mean age was 55.2 years, operative time was $30.1 \mathrm{~min}$. Complications were: 4 recurrent nerve palsies, 18 transient hypocalcemia, 1 bleeding. Parathyroidectomy is not technically demanding: in most of cases is easier to remove the gland than to find it. Still today successful parathyroidectomy relies on surgeon's experience. In fact, qPTHa allows deciding how many glands to remove, but it is still necessary to recognize which is the gland to remove. Moreover, parathyroidectomy can be followed by complications like nerve injury and bleeding. MIVAP revealed to be safe and it might also play a role in a teaching hospital. In fact, MIVAP can be more useful than other minimal invasive procedures in terms of surgical training for two reasons: it might improve surgical endoscopic skill ness and it allows better anatomical view of abnormal/normal parathyroid glands and nerves.

\section{9}

\section{A Single One Institution Experience in Ship Collision}

S. Mazzei ${ }^{1}$, F. Famà ${ }^{1}$, A. Vindigni', S.A. Villari ${ }^{1}$, C. Linard ${ }^{2}$ M.A. Gioffrè-Florio ${ }^{1}$

${ }^{1}$ Emergency Care Unit, Department of Human Pathology, University of Messina, Italy; ${ }^{2}$ Service de Anesthésie et Réanimation, Centre Hospitalier Régional, Orléans, France

Introduction: The Messina strait is a sea-area among Sicily and Calabria travelled by merchant ships, ferry-boats and hydrofoil, that links Messina to Reggio Calabria. In January 2007, antero-lateral was crush between merchant ship and hydrofoil, with 151 passengers and 4 members of crew, 4 deaths and 105 injuries were recorder. Our experience in the emergency care of 25 patients is reported.

Methods: All patients were investigated with routine blood tests, imaging techniques and classified according to AIS (Abbreviated Injury Scale) and ISS (Injury Severity Score). Those were classified as 14 green (7 M, 7 F); 9 yellow ( $5 \mathrm{M}, 4 \mathrm{~F})$ and 2 red (2 M) code. The time of crash (17:53) and first admission to hospital $(19: 31)$ was 91 minutes. 
Results: Among 25 patients, 10 presented with cranial injuries, 6 spinal injuries, 5 thoracic injuries, 3 long bones fractures and 1 patient with laceration, classified in according to worse injury. Among all patients, 18 presented with an ISS $<15,7$ with an ISS of 28 (range 17-45) and nobody with an ISS $>50$. Twelve patients were admitted to the hospital; 9 rejected hospitalization, 4 were discharged.

Discussion: Twenty-six ship-collisions occurred in Messina Strait between 1950 and 2007 were documented. Nine deaths happened. No environmental disaster happened. A careful control of this area to prevent accidents would be necessary. The aim of this study is to prove that there was a correct management of this major emergence with creation of trauma team, fast triage at the impact area and patients transport to hospital.

\section{0}

\section{Immunostimulation to Reduce Recurrences after Surgery for Anal Condyloma Acuminata}

M. Mistrangelo', S. Cornaglia², M. Pizzio ${ }^{2}$, R. Brustia, A. Mussa ${ }^{2}$

${ }^{1}$ General and Emergency Surgery II, University of Turin, Dir Prof. M. Morino, ${ }^{2}$ Surgical and Oncological

Department, University of Turin, Italy, Dir. Prof. A. Mussa

Introduction: Anogenital warts are the most common sexually transmitted disease. They cause emotional distress for its marked tendency to recurrence. Immunosuppression is an important risk factor. De la Fuente evidenciated a recurrence in $66 \%$ of immunosuppressed patients compared with $27 \%$ of immunocompetent ones.

Patients and Methods: 261 patients were submitted to a randomized prospective study consisting in surgical excision of perianal and endoanal condylomata. 139 patients were submitted to a postoperative immunostimulation for 30 days with a natural product and they were compared with 122 patients not immunostimulated. We use a product containing Echinacea, Uncaria, Tabebuja, Papaya, grapefruit and Andrographis. An informed consent was signed. HIV+ patients were excluded from the study.

Results: Follow up was performed for 6 months after surgery. Total amount of recurrences were 7.19\% (10/139) in study group, vs. $27.05 \%(33 / 122)$ in control group with a Chi Square $<0.0001$ and a significative Fisher's exact test. Sex, sexual habits, number of lesions, time to diagnosis and treatment and localization don't influence difference of recurrence in the 2 groups.

Conclusions: Immunostimulation in patients submitted to surgery for anal condylomata is useful to reduce recurrences. The use of a natural product guarantee good results without the risk of sides effects.

\section{1}

\section{Laparoscopic Diagnosis and Treatment of Primary Torsion of the Greater Omentum}

\author{
A. Palladino, S. Cecchini, R. Costi, L. Sarli, L. Roncoroni \\ Department of Surgical Sciences, Section of General \\ Surgical Clinics and Surgical Therapy, Parma University \\ Medical School, Parma, Italy
}

Purpose: Clinical presentation of primary torsion of the greater omentum is aspecific and may mimics other more common causes of acute abdomen, so, rarely allowing for a preoperative diagnosis. When etiology is unclear, diagnostic laparoscopy has been proved to be safe and effective even in emergency patients, sometimes enabling the surgeon to treat preoperatively undiagnosed conditions. The aim of this three-case-report is to evaluate effectiveness, feasibility and safety of laparoscopy in diagnosis and treatment of omental necrosis.

Methods: Three patients presented with acute but aspecific abdominal symptoms (limited peritonitis of the right iliac fossa in two cases and left flank in one), associated to nausea and fever in two cases. Two patients had neutrophilic leukocytosis. Since US and radiological findings were negative or unclear, all patients underwent diagnostic laparoscopy.

Results: In all cases, laparoscopy allowed to achieve the diagnosis, to place the operating trocars in the most convenient places, to perform a resection of necrotic omentum and to deliver the specimen by enlarging the supra-pubic port-site incision. The procedure lasted 56 minutes (range 42-76). Patients were discharged on postoperative day 3 (one case) and 1 (two). The postoperative course and follow-up (14, 42 and 60 months) were uneventful.

Conclusions: The value of diagnostic laparoscopy increases when the disease can be treated laparoscopically. The laparoscopic approach allows for the exploration of the whole abdominal cavity and the possibility to aspirate and wash the peritoneum, which are not allowed by laparotomy (unless a wide longitudinal xifo-pubic incision is performed). In the reported cases, after having diagnosed omental necrosis, the correct placement of the operative trocars at the most convenient places under laparoscopic vision facilitated the procedure. The laparoscopic resection of the greater omentum is an easy task even for inexperienced laparoscopic surgeons, allowing patients to benefit of advantages of mini-invasive approach.

\section{2 \\ First Robotical Approach to Esophageal Duplication Cyst}

A. Palladino, M. Piccoli, G.L. Melotti

Department of Surgery (head prof G. Melotti) and ACOI's Special School of Laparoscopic and Mini-invasive Surgery (director prof G. Melotti), Nuovo Ospedale S. AgostinoEstense, Modena, Italy

Purpose: The aim of this case-report is to evaluate effectiveness, feasibility and safety of robotic surgery in treatment of esophageal duplication cyst, as laproscopic and thoracoscopic approaches have just been assessed. 20th National Congress of the Italian Polyspecialist Society of Young Surgeons (IPSYS) 
Materials: A 17th years old boy with recurrent acute but aspecific abdominal symptoms (precordial pain without dysphagia, gastric pyrosis, weight loss or alvine alteration). US, CT and endoscopy showed a cystical formation of $21 \mathrm{~mm}$ between left hepatic lobe and pancreas at the esophageal thoraco-abdominal passage. Umbilectomy as only previous surgical treatment.

Methods: Pneumoperitoneum with Veress needle in left hypocondrium was performed, 1 paraumbelical optical trocar, other 4 robotical accesses and a 5th accessory trocar were positioned. Intraoperatory US and endoscopy were previously repeated. Longitudinal esophageal myotomy, enucleation of the mass and the suture of the muscle were carried out; a new endoscopical intraoperative control confirmed the absence of mucosal lesions. Patient was discharged on postoperative day 3 . The course and follow-up (4 months) were uneventful.

Conclusions: The value of robotic surgery increases when the disease can be treated robotically. The possibility offered to the surgeon to position independently the camera, its stability in the operatory field and the magnification of the images, may facilitate every kind of procedures. Above all, the robotic resection of an esophageal duplication cyst is an easy task for experienced laparoscopic surgeons, allowing patients to benefit of advantages of mini-invasive approach.

\section{3}

\section{Hemostats and Sealants in Surgery: State of the Art}

S. Panicucci, F. Filidei, M. Barlettai, F. Scassa, R. Cantisani, E. Pressi

U.O. Chirurgia Generale e d'Urgenza, Direttore

Prof. M. Seccia - Azienda Ospedaliera Universitaria Pisana

Introduction: At present, one of the most expanding field in surgery is the use of topic haemostats and sealants. To date, more than 4,000 papers are available in the literature with an exponential growth of new products and industrial firms. In spite of that, the choice and the use of these substances remain ruled more by individual rather than standardized criteria.

Methods: To offer a guideline for a safe and appropriate use of these substances the authors carried out an accurate analysis (on the Literature and 'on the field') of sealants and topical haemostats available in worldwide trade, at the same time performing an internal report for Pisa University Hospital. The main target of the study was to define a milestone in this chaotic field comparing on of efficiency, safety and handiness of different agents in elective and emergency surgery.

Results: Our report confirmed the low knowledge about rational usage of these products in general and specialist surgeons. An innovative classification of products, based on active principles, and internal guidelines for 'why, when and what' in elective and emergency surgery were defined. The study is still in progress.

Discussion: Using haemostats and sealants in surgery should be considered with the same attention and knowledge paid for other surgical tools (as sutures, staplers etc) and not as a 'nice' optional (but expensive!) toy.
104

\section{Treatment of Traumatic End-stage Faecal Incontinence with Electrostimulated (Dynamic) Graciloplasty}

\author{
S. Panicucci, G. Zocco, F. Filidei, F. Scassa, M. Barlettai, \\ M. Mazzillo \\ U.O. Chirurgia Generale e d'Urgenza, Direttore \\ Prof. M. Seccia-Azienda Ospedaliera Universitaria Pisana
}

Introduction: Restoring faecal continence after severe anorectal traumas still remain a surgical challenge. Dynamic Graciloplasty (DG) demonstrated to be a complex but reliable option in congenital and acquired incontinence (FI) refractory to conventional procedures. We report our experience with DG in 'devastating' anorectal traumas.

Methods: Since 1992, 9 patients ( $5 \mathrm{M}$; 4 F; mean age $25.5 \mathrm{yrs}$; range 14-46) have been submitted to DG, 4 for blunt and 5 for penetrating traumas. All patients received a stoma in other Institutions. Blunt traumas were due to MVAs, while penetrating injuries were due to firearm wounds (1 hunting rifle, 1 terrorist attack), sexual assault, playground accident and a MVA. Preoperative evaluation included endoanal US, NMR, manometry (EMM), dephaecography and porridge test. Postoperative evaluation was based on Williams' Scale (NWS) and EMM.

Results: 10 DGs were carried out in 9 patients. Early postoperative complications occurred in 5 (55\%), mainly for wound infection. At present, 7 patients have good restoration of continence (NWS I in $1 \mathrm{pt}$, II in 6 pts) while in 2 only a slight improvement was observed. No patients required reinterventions.

Discussion: Restoration of continence after devastating pelvic traumas needs a sphincter replacement. DG demonstrated to be a reliable option, offering an effective rate of faecal continence restoration.

\section{5 \\ Immunostimulant Effect of Withania Somnifera's Extract: A Possible Postoperative Mechanism of Action}

\section{T. luvone1, D. Parmeggiani²}

${ }^{1}$ Department of Experimental Pharmacology, University of Naples 'Federico II', Italy; ${ }^{2} \mathrm{~V}$ Division of General Surgery Second University of Naples

Introduction: Ginseng is an immunostimulant herbal medicine used to improve overall health in elderly people and to prevent diseases particularly in immunocompromised patients. However, the mechanisms underlying the immunostimulant effect of Ginseng is poorly understood. Among the several species of Ginseng, including Siberian, (Eleuterococcus), Brazilian, Peruvian and Indian Ginseng (Withania somnifera), we investigated the effect of a methanolic extract from the root of Withania somnifera (WS) on nitric oxide (NO) production and inducible NO synthase (iNOS) protein expression in macrophages.

Methods: The effect of WS was tested on murine monocyte/ macrophage $\mathrm{J} 774$ cells. Cells were incubated at $37^{\circ} \mathrm{C}$ in $5 \% \mathrm{CO}_{2} / 95 \%$ air; after $24 \mathrm{~h}$ we measured nitrite production, by Griess reaction and iNOS protein expression by western blot analysis. 
Results: WS $(1-256 \mu \mathrm{g} / \mathrm{ml})$ produced a significant and concentration-dependent increase in NO production, an effect which was abolished by $\mathrm{N}^{\mathrm{G}}$ nitro-L-arginine methyl ester (L-NAME, $3-300 \mu \mathrm{M})$, an inhibitor of iNOS, dexamethasone $(10 \mu \mathrm{M})$, an inhibitor of protein synthesis and $\mathrm{N}_{\alpha-\mathrm{p}}$-tosyl-L-lysine chloromethyl ketone (TLCK, $0.01-10 \mu \mathrm{M}$ ), an inhibitor of NF- $\mathrm{BB}$ activation, the transcription factor coding for iNOS gene. Dexamethasone did not have any effect on NO production once iNOS had been induced (i.e. $12 \mathrm{~h}$ after WS). Moreover, western blot analysis showed that WS increased, in a concentration-dependent fashion, iNOS expression.

Conclusion: These results demonstrate that WS may induce the synthesis of iNOS protein expression by acting at transcriptional level. The increased NO production by macrophages could account, at least in part, for the immunostimulant properties of WS (Indian Ginseng). Therefore it is conceivable to use Ginseng extracts for the treatment of immunocompromised patients, including post-operative ones.

\section{6}

\section{Segmental Infarction of the Greater Omentum: A Case Report}

\author{
F. Pata ${ }^{2}$, P. Mingrone ${ }^{1}$, P. Grandinetti ${ }^{2}$, E. Lanoce ${ }^{3}$, \\ P.M. Cloro ${ }^{2}$, R. Sacco ${ }^{2}$, L. Cloro ${ }^{1}$ \\ ${ }^{1}$ NN. Giannettasio' Hospital, Rossano Calabro (CS), Italy; \\ ${ }^{2}$ General Surgery, 'Magna Graecia' University, Catanzaro, \\ Italy; ${ }^{3}$ Urology, 'Magna Graecia' University, Catanzaro, Italy
}

Introduction: Idiopathic or spontaneous segmental infarction of the greater omentum (ISIGO) is an unusual cause of acute abdomen.

Methods: A 69 years old man was admitted to the our unit with pain (and rebound tenderness) in the right lower quadrant of abdomen of 3 days duration. Preoperative diagnosis of appendicitis was made. The patient was operated upon through a pararectal incision and the operation revealed a segmental infarction of greater omentum, adhering to appendix and last ileal segment. Appendicectomy, resection of necrotic omentum and last ileal segment were performed.

Results: No significant surgical complication was occurred, and patient was discharged from the hospital on the 14th postoperative day. Histological examination demonstrated necrosis of the omentum excised without involvement of appendix or last ileal segment.

Discussion: Segmental infarction of the greater omentum is a rare disease, also if rate of occurrence may nevertheless be underestimated. The clinical picture simulates an appendicitis or cholecystitis, and an accurate diagnosis is seldom made before surgery. However, resection of the affected omentum (currently possible also by laparoscopic approach) is curative in $100 \%$ of cases, and, once identified, it should be completely excised to prevent formation of adhesions and possible sepsis.

\section{7}

\section{Surgical Prevention of the SSIs}

M. Perone, I. Donzelli, S. Di Lauro, M. Di Giuseppe, R. Di Micco, G. Orabona, G. Grillo, C. de Werra

University of Naples 'Federico II'- General, Oncological, Geriatrical and Advanced Technologies, Surgical

Department - General Surgery Area (Dir. Prof. P. Forestieri)

Introduction: An accurate observance of the principles of treatment of the surgical wound dictated by the CDC 1999 and the application of the knowledges about the patogenesis of the SSIs can prevent the great majority of the infectious complicances.

Materials and Methods: Pre-operating phase: It useful to reduce to the least one the tied up factors of risk of the patient and the times of hospitalization, to check the infections of the medical and paramedical personnel. The antiseptic shower the evening before the operation, the hygiene and the antisepsis of the site of incision and the trichotomy, only if it needs and using cutter clipper, and finally the antibiotic prophylaxis are useful. Operating phase: the operating room might have an effective conditioning and environmental hygiene and the staff must respect all the tools' preparation protocols. The health care staff must have a suitable attire, have to wash their hands for at least 5 minutes with appropriate antiseptic solution (clorexedina and iodiopovidone), they should avoid to operate if they have any skin hands or arms lesions; they must wear two pairs of surgical gloves to avoid the active and passive contamination. The surgical technique might be suitable to the type of intervention and must apply some essential rules. The totality of the SSIs, in fact, is almost acquired in the operating room during the intervention. It is essential to take care of the vascularization of the shreds, the stitches should be not too closed to avoid ischemia, it is useful to prefer non absorbable syntethic monofilament, new sutures with antiseptic threads, accurate haemostasis, suitable cauterization (to avoid necrosis), aspiration drainages to eliminate dead spaces; miniinvasives technical should be preferred.

Results and Conclusion: Our experience (Kir Nos study) of the application of above mentioned procedures demonstrates that SSI can be prevented and reduced, and that an accurate surgical tecnique is the best way to obtain it.

\section{8}

A New Partially Resorbable Prosthesis 4DDOME ${ }^{\circledR !}$ for Inguinal Hernia (IH) Repair. Preliminary Results

\author{
E. Piazzese1, G.I. Mazzeo', S. Galipò', F. Fiumara², \\ L.G. Angiò? ${ }^{2}$. \\ ${ }^{1}$ Unità Funzionale di Chirurgia Laparoscopica, Clinica San \\ Camillo, Messina; ${ }^{2}$ Scuola di Specializzazione Chirurgia \\ Generale I, Università degli Studi di Messina
}

Introduction: IH are the most common conditions for which primary care physicians refer patients for surgical management.The main problems of modern IH repair concern the search of ideal technique to prevent recurrence and to reduct hospital staying and 
complications. The postoperative pain (1-17\%) seems to be the most important cause of prolonged convalescence and is partly related to the dissection technique, the excess of prosthetic material and fixation techniques. For such considerations an ideal prosthesis should combine strength with minimal material bulk. A new semi-resorbable reinforcement prosthesis (Poly-L-Lactic-Acid + light-weight polypropylene) denominated $4 \mathrm{DDOME}{ }^{\circledR}$ was evaluated for $\mathrm{IH}$ repair.

Methods: 10 unselected male patients (age range 19-65) underwent to surgery for $\mathrm{IH}$ repairs using $4 \mathrm{DDOME}^{\circledR}$ prosthesis second modified Lichtenstein technique. There were 2 type IV, 3 type III and 5 type II hernias (Nyhus classification). 4 IH repairs were performed under local anesthesia, 6 patients requested a general anesthesia. Postoperative data were analyzed with a maximal follow-up of 8 months.

Results: Mean hospital stay after surgery was 1.5 days. There were no perioperative deaths, postoperative complications, persistent pain, or recurrences. Postoperative imaging did not demonstrate any shrinkage of the $4 \mathrm{DDOME}^{\circledR}$ prosthesis, no contact inflammatory reaction.

Conclusion: From the results of our preliminary experience we can affirm that the employment of $4 \mathrm{DDOME}^{\circledR}$ prosthesis is reliable and well tolerated for $\mathrm{IH}$; the further validation could derive from a longer follow-up and from comparison with some other prosthesic materials.

\section{9}

Clinical Application of a Poliurethan Flexible Foam Dressing (PFFD) and Low Molecular Weight Heparin (LMWH) in Nonhealing Ulcers of the Lower Limbs (NULL)

\author{
E. Piazzese1, G.I. Mazzeo', S. Galipò’, F. Fiumara², \\ L.G. Angiò ${ }^{2}$ \\ ${ }^{1}$ Unità Funzionale di Chirurgia Laparoscopica, Clinica San \\ Camillo - Messina; ${ }^{2}$ Scuola di Specializzazione Chirurgia \\ Generale I, Università degli Studi di Messina
}

Introduction: NULL are a common, severe, and expensive complication threatening life and limb in patients with diabetes mellitus (DM), distal arterial ischemia (DAI), venous cronic insufficency (VCI); often offer a multifactorial aetiology and thus they show a difficult therapeutic approach.

Methods: 15 patients with NULL ( 9 female -6 male, range 58-85 years) have been treated with LMWH (local subcutaneous injection of $2500 \mathrm{UI}$ of dalteparin) associated with PFFD (1-2 cm thick related to the amount of fluide exudate). The skin wounds found their origin in different pathologies: DM (6 cases), DAI (4 patient), VCI ( 5 cases). The therapeutic procedure, was effected every 48 hours.

Results: All patients demostrated a good healing of the wound with acceptable functional and cosmetic result. The healing time rate has been variable, ranging from a maximum of 7-12 weeks (methabolic disease) to a minimum of 4-6 weeks (vascular disease). No skin allergies were observed. A single disadvantage was represented by a local increasing pain ( 2 case), moreover easily controlled with analgesic i.m.

Conclusions: Present preliminay study indicate that LMWH associated with PFFD improves the outcome of NULL. The results infact show that LMWH positively influences the healing process of chronic ulcers, possibly by improving the capillary circulation in the ulcer margin, in spite of an unchanged arterial and total skin microcirculation of the region; PFFD, instead, should interfere the healing process carring out a continuos mechanical debridement and a fluid exudates balance management.

110

\section{The Employment of an Original Ring Handle Forceps in Anterior Repair of the Primitive Inguinal Hernia}

\author{
L.G. Angiò, E. Piazzese, F. Fiumara, V. Pacilè, \\ A. Bonsignore \\ ${ }^{1}$ Scuola di Specializzazione in Chirurgia Generale I, \\ Università degli studi di Messina, ${ }^{2}$ Unità Funzionale di \\ Chirurgia Laparoscopica - Clinica San Camillo Messina
}

The Authors propose the employment of an original dissecting and ribbon-carrier bevelled ring handle forceps in inguinal prosthetic tension-free hernioplasty with mini-inguinotomy. The surgical instrument, of stainless steel and 16 centimetres long, consists of two jaws with ring handle, ratchets and box lock nearly identical to those of common surgical instruments; is slightly curved in his distant part, where shows a large bevelled end and a large seizing, entirely original. The peculiar surgical instrument gives possibility to operator to make easily and delicately the atraumatic mobilization in proximity to the pubic tubercle of the spermatic cord from the back wall of the inguinal passage, in place of the index finger hook-shaped who, used roughly and by pulling in large incisions, cannot be used instead in the mini-incisions because of limited available space in the surgical site. On end, the new ring handle forceps allows to keep in suspension the spermatic cord by rubber ribbon more simply and rapidly than the usual big ligature-carrier. On the whole, the dissecting and ribboncarrier bevelled ring handle forceps, whose the Authors use habitually the prototype in inguinal prosthetic tension-free hernioplasty with mini-inguinotomy, allows the execution of easy, prudent, elegant, precise, effective and above all safe surgical gestures.

\section{1}

\section{Perforation of the Small Bowell (SB) caused by Metastasis from Lung Cancer (LC): Case Report}

\author{
F. Fiumara', L.G. Angiòn ', E. Piazzese², G.I. Mazzeo², \\ S. Galipò ${ }^{2}$ \\ ${ }^{1}$ Scuola di Specializzazione Chirurgia Generale I, \\ Università degli Studi di Messina; ${ }^{2}$ Unità Funzionale di \\ Chirurgia Laparoscopica, Clinica San Camillo - Messina
}

Introduction: LC, the first cause of death for tumors, can metastasize to any organ in the body; however, metastasis to the SB are a relatively rare event $(1.1 \%)$. There is often a poor symptomatology and diagnosis is usually based on autopsy. Yet, sometimes, the first and only demonstration of the secondary illness is a surgical 
complication, whether it be a haemorrhage, an occlusion or a perforation (infrequently).

Case Report: Case report was a 56-year-old man who had LC and submitted to right superior lobectomy. Because he showed progressive disease (bony and cerebral metastasis) after induction chemoradiotherapy, he started to receive best supportive care alone. Six months after initial diagnosis, he complained of an abdominal pain and intestinal perforation was diagnosed by a X-ray and CT-scan. An emergency surgery was undertaken and the surgical findings showed perforation by jejunum; intestinal resection was performed and the histologic findings of the resected specimen consisted in SB metastasis from LC. Although the patient recovered sufficiently, he died in a hospice two months after the operation.

Conclusion: Our case report has confirmed the data presents in literature; even if SB metastasis from LC is rare in clinical situations, nevertheless, if a patient with LC complains of abdominal pain, it is important to consider the possibility of abdominal metastasis. The clinical presentation of SB metastasis of LC may represent a terminal event if not recognized and resolved; the surgeons should be aware of the poor prognosis of such patients and perform only the minimal surgery required.

\section{2}

\section{Low-Grade Fibromyxoid Sarcoma (LGFMS) of the Thigh: Clinicopathologic Case Report with Review of the Literature}

\author{
F. Fiumara1', L.G. Angiòn', E. Piazzese², G.I. Mazzeo², \\ S. Galipò ${ }^{2}$ \\ ${ }^{1}$ Scuola di Specializzazione Chirurgia Generale I, \\ Università degli Studi di Messina; ${ }^{2}$ Unità Funzionale di \\ Chirurgia Laparoscopica, Clinica San Camillo - Messina
}

Introduction: LGFMS is a rare soft-tissue tumor with a deceptively benign histologic appearance affecting predominantly young adults; has a predilection for involving deep soft-tissues of the thigh, inguinal region, or chest wall. Local postsurgical recurrence and metastases (lungs and bone) are frequently seen. The surgical management, histopathologic findings, and biological behavior of LGFMS have been outlined in the literature.

Case Report: A 73-year-old woman presented with a 2-year history of a painless slow-growing mass in the left thigh. CT and MR images were obtained, demonstrating a well-defined soft-tissue mass measuring approximately $10 \mathrm{~cm}$ located among the fibers of the quadriceps muscle without signs of peri-tumoral edema in adjacent subcutaneous tissues or muscles. The lesion was surgically removed with negative margins. Macroscopically, the specimen consisted of a soft-tissue tumor, measuring $11.5 \times 9.5 \times 7 \mathrm{~cm}$; the cut sections showed a firm, grossly circumscribed, and lobulated mass, with a white color and glistening appearance. A histopathological diagnosis of LGFMS was made. After the surgery, the patient was treated with radiotherapy without chemotherapy, has been observed for 16 months and is alive without the evidence of disease.

Conclusion: Although LGFMS is a rare entity, it is reasonable to speculate that it is probably more common than reported in the medical literature due to the likelihood of misdiagnosis with other low-grade fibrous or myxoid soft-tissue neoplasms; recognition of this lesion is important for proper surgical treatment and long term follow-up because metastases may occur many years later. Due to the rarity, the role of chemotherapy and radiotherapy remains unknown.

\section{3}

\section{The Role of Experimental Surgery in Education and Research of General Surgery Trainees}

\section{E. Poli, G. Lissidini, A. Gurrado, D. Lardo, L. Nacchiero, L. Ruggiero, M. Signorile, M.P. Valentini, M. Testini}

Department of Applications in Surgery of Innovative Technologies. Section of General and Thoracic Surgery, University Medical School of Bari, Italy

Introduction: Medium-size animals are considered to be important for education and training in medical school to master the skill required in surgical treatment. Moreover, experimental surgery played a key-role in surgical education in Italy, Europe and North America since 1960s and 1970s, but in the early of 1980s public opinion began to exert stern opposition in protest against experimental research to protect the animals from suffering.

Methods: We performed three experimental models to demonstrate the different biochemical behaviour of anastomized small vs. large rabbit bowel, the different postoperative motility of the same tissues, and the liver function modifications following bile duct ligature in the rat. All these protocols were approved by the Ethical Committee.

Results: Experimental surgery was performed on 45 male New Zealand rabbits in the first, and 60 in the second study; 40 male Wistar rats were employed to perform the third study. The results of these experimental papers were published on four ISI-journals with impact factor.

Discussion: Experimental surgery, especially in the microsurgical approach, is fundamental in surgical training. Young surgeons can perform surgical procedures and easily get familiar with surgical instruments and sutures. Moreover, the organization of a research protocol is highly useful also in the medical education of residents in general surgery. The limit is often represented by the approach to complex surgical experimental procedures and models by trainees with a low level of surgical skill. In this study we propose a learning model of experimental surgery for the residents.

\section{4}

\section{Systematic Reviews according to the Cochrane Collaboration Criteria in General Surgery}

\author{
C. Rispoli, N. Rocco, A. Antonino, V. Girardi, M. Celiento, \\ M. Persico, B. Amato
}

Department of General Surgery, University 'Federico II' of Naples, Chairman: Prof G. Persico

Systematic Reviews (SR) have been performed in natural sciences since long ago. The cumulative scientific information is the basis to 
improve knowledge and stimulate new ideas. Progress in science are highly dependent on a systematic approach of the known literature, it is the necessary way to rapid development and large diffusion of scientific knowledge. In scientific medicine SR are essential with special respect to the transfer of knowledge in applied sciences such as clinical practice. The Cochrane Collaboration has defined criteria to perform standardized and independent SRs highlighted below; those criteria could also be applied to general surgery. First, define the surgical problem. Second, perform a library search of all relevant papers starting from electronic databases, conference proceedings and, if needed, unpublished data. Third, formulate an evaluation strategy to eliminate sources of bias, classifying the validity of any single study. Fourth, put together screened information in a systematic way identifying the statistical and clinical significance of the results with attention to surgeons, researchers and patients. In performing such path to produce SRs in surgery, researchers will have to face a specific problem that is the craftsmanship of surgical procedures; this is a great confounding factor in pooling data from surgical studies.

\section{5}

\section{Locoregional and Distant Recurrences after Curative Surgical Treatment of Colon Cancer}

\author{
A. Mingione, A. Antonino, C. Rispoli, L. De Magistris, \\ L. lannone, B. Amato \\ Department of General Surgery, University 'Federico II' of \\ Naples
}

Introduction: The aim of this study is to identify patient's or tumor's characteristics which mostly influence recurrence risk after a radical surgical operation for colon cancer.

Methods: In this study, we report all patients with colon cancer who underwent laparotomic surgical treatment from 1998 to 2002. The total number of patients was 115 with average age of 65.2 ; male to female ratio was 1.61. As regards the variations due to pre-operatory staging and tumor localization, our patients underwent: 34 right emicolectomy, 80 left emicolectomy, 1 transverse resection. According to the stage of tumor, an adjuvant chemiotherapic treatment was associated to the surgical one. During a 5 years follow-up we investigated the locoregional or distant recurrence rate. We considered as locoregional recurrences that ones which developed in the same abdominal region of the primary tumor; the most common sites of distant metastasis were liver and lung.

Results: Our first results show that both locoregional recurrence and distant metastasis rates depend mostly on the extension of tumor $(\mathrm{T})$, nodal involvement $(\mathrm{N})$ and grading $(\mathrm{G})$ of the primary tumor.

Discussion: These results show the fundamental role of the follow-up and its attention to all the factors which mostly influence recurrence risk after a radical surgical operation for colon cancer.

\section{6}

\section{New US-color-Doppler Criteria in the Diagnosis of Subclinical Varicocele}

M. Celiento, C. Cirillo, T. Cacciapuoti, A. Mingione, C. Rispoli, V. Piscitelli

Department of General Surgery, University 'Federico II' of Naples

Introduction: Varicocele is a common pathology, often associated with infertility. There's no correlation between varicocele size and degree of injury to testicular tissue responsible for infertility. The aim of this study is to determine new criteria in the diagnosis of subclinical varicocele in infertile patients.

Methods: A total of 124 testes in 62 patients who were infertile and had no varicocele on physical examination were studied by color Doppler ultrasonography (CDUS) with classical and new criteria. Population proportion difference test was used to compare two methods.

Results: 46 were accepted as varicocele negative in the same testes units in both methods. 78 varicocele positive with new criteria while 72 of these 78 testes were diagnosed also varicocele positive with the classical criteria

Discussion: Because a gold standard that defines the presence of a subclinical varicocele has not yet been established, new criteria may be needed. However, our new criteria did not add new information to the diagnosis of subclinical varicocele in infertile patients.

117

\section{Surgical Treatment and Prognostic Factors in Colorectal Cancer}
A. Antonino, A. Braun, A. Mingione, C. Cirillo, V. Girardi, D. De Vito, V. Di Maio
Department of General Surgery, University 'Federico II' of Naples

Introduction: The colorectal cancer is the second cause of death in patients with neoplasm in the Western World. The aim of the study is to give our contribution to improve patients care, with special attention to prognostic factors, and consider what can influence positively the quality of life in the immediate postoperative course.

Methods: In this study, we report all patients with colorectal cancer who underwent laparotomic surgical treatment from 1996 to 2003. The total number of patients was 226 with average age of 65.6 ; male to female ratio was $1.57: 1$. According to the stage of tumor, an adjuvant radiotherapic and/or chemiotherapic treatment was associated to the surgical one. As regards the variations due to pre-operatory staging and tumor localization, our patients underwent: 3 total colectomy, 57 right emicolectomy, 137 left emicolectomy, 6 Hartmann resections, 19 Miles resections, 4 transverse resection.

Results: Our first results show that 5-year overall survival is particularly influenced by the stage and the factors which directly or indirectly affect the stage of the primary tumor.

Discussion: Especially three are the prognostic factors that affect significantly overall survival and that need to be considered during patients evaluation in a follow-up for malignant tumor: stage, grading and nodal involvement. 


\section{8}

Cystic Pancreatic Neuroendocrine Neoplasm to Uncertain Malignant Potential: Report of Two Case

\author{
R. Ballarin, M. Masetti, F. Di Benedetto, N. De Ruvo, \\ R. Montalti, G. Arzu, A. Romano, G. Guerrini, S. Di Sandro, \\ M.G. De Blasiis, M. Spaggiari, C. Longo, N. Smerieri, \\ G. Rompianesi, G.E. Gerunda \\ Centro trapianti fegato e multiviscerale, Modena
}

Introduction: Neuroendocrine tumors of the pancreas (NETP) are uncommon tumors occurring in approximately $0.4-1$ in $1,00,000$ people and representing only $1-2 \%$ of pancreatic neoplasms. NETP usually appear radiologically as solid tumours although rarely may manifest as cystic lesions of the pancreas. Frequently, cystic NETP cannot be differentiated pre-operatively from other cystic pancreatic tumours. The treatment options for NETP have evolved over the past decade, the optimal treatment for all of these lesions is radical surgical resection whenever possible.

Method: We report about 2 cases of cystic pancreatic endocrine tumors.

Discussion: Cystic changes in NETP are a rare phenomenon of uncertain origin and involve $2-8 \%$ of resected pancreatic cyst. These tumours are mainly localized in the distal part of the pancreas and most of them are non-malignant and non functional. The importance to establish a diagnosis for cystic lesions of the pancreas is clearly to define benign lesion versus premalignant lesion or frankly malignant conditions. However, cystic NETP are difficult to diagnose preoperatively because the majority of these tumors are non-functional, and cross-sectional imaging is notoriously unreliable in distinguishing between the various pancreatic cystic lesion and cystic NETP frequently mimick pseudocysts or other pancreatic cystic neoplasm. The pathophysiology of cystic NETP remains controversial and several hypotheses have been proposed to explain its formation.

Summary: Cystic NETP represent a subgroup of pancreatic cystic and neuroendocrine tumors with malignant potential. Their high resectability rate further supports the role of surgical exploration and resection in the treatment of pancreatic cystic neoplasm.

\section{9}

\section{Useful Parameters to Identify Patients with Defaecatory Disorders who might Benefit from Pelvic Floor Rehabilitation}

\author{
L. Brusciano, C. Sagnelli, G. del Genio, G. Rossetti, \\ F. Pizza, S. Tolone, L. Barra, A. del Genio \\ Chirurgia Generale, I chirurgia SUN, Napoli
}

Introduction: Obstructed defecation is due to both organic and functional causes. Among functional causes the pelvi-perineal dissynergia (PPD) plays a remarkable role. The PPD is a complex syndrome characterized by absent or delayed relaxation or paradox contraction of puborectalis muscle during the defecatory act, associated to an asynchronism between abdominal and pelvic muscle districts.
Methods: All patients are subjected to the neurophysiactric exam, the clinical exam, perineal clinical examination and dynamic defecography, anorectal manometry, endoanal and dynamic perineal ultrasonography.

Results: The neurophysiactric exam allows to estimate the lumbar lordosis. The clinical exam evaluates the perineal defence reflex and the action of abdominal muscles due to an increase of pressure into the abdominal cavity and the possible association with incontinence to gas and/or urine. The perineal clinical examination helps us to estimate the following parameters: the muscle response to abdominal pressure increase, the presence of eventual perineal muscular synergies and the level of tone and fatigue of the elevator of anus and the pubo-coccigeum.

Discussion: These physiatric data, along with those obtained by morfo-functional tests may help us to highlight patients affected from defecatory disorder and to address them to pelvic floor rehabilitation.

120

\section{Radioguided Sentinel Node Identification in Colonic Cancer. Preliminary Study on Method Validation}

\author{
A. Sanna1, P. Marinelli², N. Borsato ${ }^{2}$, D. della Libera ${ }^{3}$, \\ L. Bittesin ${ }^{3}$, M. Schiano di Visconte', F. Tramonte', \\ G. Munegato \\ ${ }^{1}$ U.O. di Chirurgia Generale, ${ }^{2}$ U.O. Medicna Nucleare, \\ ${ }^{3}$ U.O. di Anatomia Patologica, Ospedale 'S.M. Battuti' \\ Conegliano-ULSS 7, Pive di Soligo (TV), e-mail:and_sanna@ \\ yahoo.in
}

Introduction: The principal role of sentinel node sampling and ultrastaging in colon cancer is enhanced staging accuracy. The purpose of this study was to validate at radioguided sentinel node identification (SNL).

Materials and Methods: Between October 2005 and December 2006. Fifth-teen unselected patients with colon cancer underwent ex vivo sentinel node identification. Ten minutes before surgical resection, was injected sub-serosal around tumor $40 \mathrm{MBq} 99 \mathrm{mTc}$. Immediately after surgical laparoscopic standard resection, the specimen performed at conventional schintigram with a gamma-camera. The sentinel node was identified and then was subsequently stitch-labeled; the others (nonSLN) were dissected away. The labeled SN were stained with hematoxylin and eosin and cytokeratin immunostaining was performed.

Results: At least one SLN was identified in 12 of 15 enrolled patients, and 2 were labeled 2 . A total of 16 hystologically confirmed LNs were examined. The T classification were $53.4 \%$ (8) T1, 33.5\% (5) $\mathrm{T} 2,13.4 \%$ (2) T3. Immunoistochimical staging reveled a metastasis in the sentinel lymph node of 3 patients. Two with other node positive and one (pT3) other LN were negative by conventional methods. Moreover, $6.6 \%$ ( 1 of 15 ) of the patients, classified as $\mathrm{pN} 0$ by routine histopathology, revealed micrometastases in the SLN.

Conclusion: Radioguided sentinel node identification in colonic cancer is a feasible technique with a high SLN identification rate. The contribution of SLN to conventional nodal staging of colon cancer patients is still unspecified. Further studies must clarify the clinical impact of these findings in terms of prognosis and the indication of adjuvant therapy. 
121

\section{Breast's Nodule as a Metastatic Amelanotic Melanoma... 10 Years Later}

\author{
M. Bortl, A. Sartori, G. Crisman, G. Trevisan, C. Gatti \\ Chirurgia Generale
}

Malignant melanoma can develop from a preexisting nevus or de novo, in patients with or without a familiar history of melanoma. A malignant melanoma can metastasize anywhere on the entire body surface, although metastasis are more common in the proximity of the primary lesion. However, a cutaneous metastasis can be the first sign of the disease because of the regression of the previous melanoma, or because of the misdiagnosis of a preexisting nevus or because of the primary lesion is situated in a so-called 'special site' (e.g. mucous membranes, eyes, etc.). We report a case of a 52-year-old woman who referred the presence of a palpable breast nodule of $27 \mathrm{~mm}$ diameter, with a mammographic and ultrasound-imaging strongly suggestive for a breast malignant lesion. After a first clinical examination, we decided to repeat the ultrasound exam, with an adjunctive agobiopsy. From the cytological and immunophenotypical examination of the specimen, we could make the diagnosis of metastasis of amelanotic melanoma in a patient with an amelanotic cutaneous lesion regressed 10 years before.

\section{2}

\section{Therapeutic Strategies for the Treatment of Perianal Fistulas in Crohn's Disease: Infliximab, Surgery or Both?}

\author{
G. Sciaudone, C. Di Stazio, P. Limongelli, I. Guadagni, \\ F. Selvaggi \\ I Division of General Surgery Second University of Naples
}

Introduction: Approximately $30 \%$ of patients with Crohn's disease $(\mathrm{CD})$ develop perianal fistulae. Infliximab, a murine chimeric monoclonal antibody against TNF- $\alpha$, is approving for the treatment of fistulizing $\mathrm{CD}$, with a median duration of fistula closure of almost 3 months. Aim of this study was to compare the outcome of management for fistulizing $\mathrm{CD}$ with infliximab alone, surgery alone or surgery plus infliximab.

Methods: Thirty four fistulizing Crohn's patients were randomized. Eleven patients (Group-A) received infliximab alone $(5 \mathrm{mg} / \mathrm{kg}$ at $0,2,6$ weeks), 11 (Group-B) underwent surgery alone (fistulotomy or seton) and 12 (Group-C) received a combination of surgery and postoperative infliximab therapy. Rate and time of healing of perianal fistula, recurrence and time to relapse were evaluated at a median follow-up of $18.6 \pm 7.8$ (range 5-38) months.

Results: Group-C patients had a closure rate of $75 \%$ in comparison to $63.6 \%$ and $72.7 \%$ of group A and B respectively. Time to healing of fistula was significantly shorter in Group-A ( $2.9 \pm 1.0$ months; $\mathrm{p}<0.05)$ and $\mathrm{C}(3.0 \pm 0.9$ months, $\mathrm{p}<0.05)$ patients compared to Group-B (4.6 \pm 0.9 months). Among patients with a complex fistula, Group-C showed a significantly longer mean time to relapse $(11.1 \pm 1.0$ months; $p<0.05)$ than Group-A $(2.3 \pm 0.4$ months $)$ and $\mathrm{B}(3.6 \pm 0.5$ months $)$.
Conclusion: This study showed better outcome in the group treated by a combination of infliximab with surgery. These patients experienced shorter time to healing of fistula and significantly longer mean time to relapse of complex fistula.

\section{3 \\ Efficacy of Infliximab in Severe Ulcerative Colitis: Case Report}

G. Sciaudone, C. Di Stazio, I. Guadagni, F. Selvaggi

I Division of General Surgery Second University of Naples

Introduction: $15 \%$ of pts with ulcerative colitis (UC) experiences a severe attack (diarrhoea $>6$ movements/day, fever $>37.7^{\circ} \mathrm{C}$, tachycardia, ESR $>30 \mathrm{~mm} / \mathrm{h}, \mathrm{Hb}<10 \mathrm{~g} / \mathrm{dl}$ ). Infliximab (IN) has been approved for the treatment of moderate-to-severe refractory UC. We report the case of a pt with severe UC candidate to surgery, in which IN gave rise to prompt improvement of clinical condition.

Case: Female, 18 years-old with UC lasting from 8 months. Remission was achieved with iv corticosteroids administration, but steroids dependence was observed. She was admitted to hospital with fever, hr $110 \mathrm{p} / \mathrm{m}$ ', abdominal pain, bloody diarrhoea $(17-20 \mathrm{~m} / \mathrm{d})$ and weight loss of $5 \mathrm{~kg}$. Hb was $6.1 \mathrm{~g} / \mathrm{dl}$, ESR $60 \mathrm{~mm} / \mathrm{h}$, abdominal x-ray showed moderate dilatation of the colon $(\varnothing 4-5 \mathrm{~cm})$. The pt received blood transfusions, total parentheral nutrition, metrhonidazole $1,500 \mathrm{mg} /$ day, ceftazidime (3 g/day), prednisolone $(1.5 \mathrm{mg} / \mathrm{kg})$, omeprazole ( $40 \mathrm{mg}$ /day), human albumin $20 \%$ (300 ml/day). Pt didn't responded to 5 -days therapy and IN $(5 \mathrm{mg} / \mathrm{kg}$ iv) was administered to avoid surgery. After $24 \mathrm{~h}$ from IN infusion pt showed a drastic reverse of clinical condition and surgery was averted. The pt was discharged after 7 days and standard IN induction protocol was carried out. During follow-up (16w) pt showed further improvement of general condition.

Conclusion: IN therapy is safe and effective in avoiding surgery for pts with refractory UC.

\section{4 \\ Use of Biological Materials for Hernia Repair in Contaminated Fields}

S. Severi, F. Catena, L. Ansaloni, F. Gazzotti, F. Coccolini, L. D'Alessandro, A. Pinna

General, Emergency and Transplant Surgery Department, St.Orsola-Malpighi University Hospital, Bologna, Italy

Introduction: Although polypropylene mesh are the preferred materials for hernioplasties, the use of these prostheses in contaminated fields is debated. In complicated hernias contaminating surgical procedure are often performed and the use of nonadsorbable synthetic materials is controindicated because of the risk for colonization and chronic infection of the mesh. In case of polypropylene meshes infection also the hernia repair can be problematic. In order to answer to these disadvantages, porcine materials (small intestinal submucosa and porcine dermal collagen) have recently been used in humans for hernia repairs. Aim of our study is to evaluate the safety 
and efficacy of incisional, inguinal and femoral hernia repair using porcine small intestinal submucosa, as a mesh, in complicated cases with contamination or infection.

Methods: A prospective study of hernia repair of complicated inguinal, scrotal-inguinal, crural and incisional hernias with contamination/ infection using porcine small intestinal submucosa grafts was carried out at the Department of General, Emergency and Transplant Surgery of St Orsola-Malpighi University Hospital. Patient demographics, fascial defect size, comorbidities and complications were recorded.

Results: From June 2001 up to now 19 patients were submitted to Lichtestein or Rutkow repair of complicated/infected inguinal/ crural hernias or complicated/ infected incisional hernia repair using small intestinal submucosa mesh. In all patients a contaminated surgical field was demonstrated with a positive microbiological culture. There were not major intraoperative or postoperatively complications related to the hernia repair.

Conclusions: Hernioplasty using the small intestinal submucosa in complicated cases with contamination has a promising safety and efficacy.

\section{5}

\section{Total Gastrectomy: Resumption's Valuation of the Intestinal Transit}

\author{
F. Tovecci, R. Compagna, P. Sorrentino, A. Bellino, \\ B. Amato \\ Department of General Surgery, University 'Federico II' \\ Naples, Chairman: Prof. G. Perisco
}

Introduction: Total gastrectomy imposes the intestinal reconstruction, that's cause many techniques have been studied: we can divide them in two categories, with duodenal transit preservation and without it. This study considers two groups of patients that have been treated using differentes techniques:

- Esophagojejunostomy of Roux.

- Esophagojejunoduodenostomy with the interposition of an ansa.

Methods: In 10 patients, subyected to total gastrectomy for a gastric carcinoma, was valued the duration of the intestinal transit by means of the administration of a barium meal to verify the causes of malabsorption in these patients. After a first abdominal radiographic exam, we checked the abdomen every 30 minutes till the appearance of the m.o.c. connected with the colon.

Results: Our study shows that the duration of the intestinal transit after total gastrectomy results quickened with middle values of visualization of the colon around $2 \mathrm{~h}$ and a half. Occurred $2 \mathrm{~h}$ when we executed an Esophagojejunoduodenostomy with the interposition of an ansa and $1 \mathrm{~h}$ and a half in case of the Esophagojejunostomy of Roux.

Discussion: Esophagojejunoduodenostomy with the interposition of an ansa is considered an efficacy technique to reach an intestinal duration transit very much alike to the physiological one. This method has some advantages like early resumption of general condition and a better gastric functionality.
126

\section{Superior Mesenteric Artery Syndrome: A Case Report and a Review of the Related Literature}

F. Torelli, D. Izzo, G. Izzo, A. Cosenza, A. Renzi,

A. Brillantino, M. Schettino, N. Di Martino

VIII Servizio di Chirurgia Generae e Fisiopatologia

Gastroenterologica Seconda Università degli Studi di Napoli

Background: Superior Mesenteric Artery Syndrome (SMAS) is related to compression of the third segment of the duodenum by the superior mesenteric artery, in association with a massive reduction of the aorto-mesenteric angle (AMA).

Methods: The patient underwent a contrastographic study of the upper digestive tract, EGDS, and angio-RM that showed a reduction of AMA $\left(15^{\circ}\right)$. The operation was performed by median laparotomy, sectioning the ligament of Treitz. A duodeno-jejunal side-end Rouxen-Y mechanical anastomosis was performed.

Results: Our report complies with the literature data, both from an epidemiological and clinical viewpoint. Surgery should be considered in patients with recurrent symptoms.

Conclusions: When symptoms of obstructed upper bowel transit occur, SMAS should be considered and consequently properly investigated. Regarding surgery we suggest that sectioning the ligament of Treitz, is the key surgical step. It should be associated with a duodeno-jejunal by-pass to grant, anyway, a good transit trought the inferior duodenal knee.

\section{7}

\section{Stapled Transanal Rectal Resection (STARR) to Treat Obstructed Defecation Syndrome: Clinical and Functional Result in 200 Patients}

D. Izzo, F. Torelli, G. Izzo, A. Cosenza, A. Renzi,

A. Brillantino, M. Schettino, N. Di Martino

VIII Servizio di Chirurgia Generae e Fisiopatologia

Gastroenterologica Seconda Università degli Studi di Napoli

Background: Obstructed defecation syndrome (ODS) is a common clinical problem. The aim of the study was to evaluate the efficacy and safety of stapler trans anal rectal resection (STARR) in patients with ODS caused by rectal intussusception (RI) a/o rectocele (RE).

Methods: All the patients underwent STARR for ODS caused by RI a/o RE. Preoperatively, all the patients underwent anorectal manometry (ARM) and cinedefecography (CD). The grade of ODS was assessed using a new obstructed defecation syndrome score (ODS-S). All the 12 and RI a/o RE, were enrolled. Patients were \pm patients with an ODS-S followed-up by ARM and CD at 3 months. Furthermore a 6-month follow-up was obtained by a telephone/mail questionnaire. 
Results: 187 (93.5\%) females on 200, 11.8 years, underwent STARR between June 2002 and February \pm pts, mean age 51.4, 0.9. RI was present in $171(85.7 \%)$ and RE \pm 2004 . Preoperatively, ODS-S was $14.3,14 \mathrm{~mm})$ in $187(93.7 \%)$ patients. No major post-operative complications $\pm(36,7.1 \mathrm{hrs}$. RI disappeared in $86(77 \%) \pm$ occurred. The length of hospital stay was $21,1.7 \pm$ and RE in $89(79.4 \%)$ patients. At 6 month follow-up ODS-S was $5.8(\mathrm{p}<0.0001)$. Successful outcome was achieved in $161 / 180(89.4 \%)$ patients.

Conclusions: STARR seems to be an effective and safe procedure to treat ODS caused by RE a/o RI. A longer follow-up and a larger number of patients are needed to confirm these results.

\section{8}

\section{Learning Curve in Bariatric Surgery. Results and Early Complications of First 100 Cases of a Young Surgeon}

\author{
V. Pilone, A. Formato, S. Tramontano, G. Ricco, \\ G. Palumbo, P. Forestieri \\ Dipartimento di Chirurgia Generale, Geriatrica, Oncologica \\ e Tecnologie Avanzate - Università degli Studi di Napoli \\ "Federico II"
}

Introduction: Laparoscopic bariatric surgery has been proven to be a technically challenging set of operations that requires a long learning curve. There may a learning curve specific also to Laparoscopic Gastric Banding (LAGB) placement.

Methods: A retrospective analysis was performed on the initial 100 consecutive LAGB placements, performed by a young surgeon. There were 27 men and 60 women, with 39.6 mean age (range 14-66), and preoperative BMI $41.7 \mathrm{~kg} / \mathrm{m}^{2}$. We reviewed of these patients conversion to laparotomy, hospital length stay, reoperation, weight loss, mortality, complications and re-operations.

Results: Between May of 2006 and May of 2007, we performed our first 100 LAGB. Two of them were re-banding. All operations were executed with a laparoscopic 4-trocars technique; we did not observe conversion to laparotomy. Mean operative time was 64 minutes (range 40-110), and mean postoperative hospital stay was 1.2 days (range 1-6). Comparing the first and the second fifty cases, operative time decreased significantly $(\mathrm{p}<0.005)$. Mean weight loss was $32.4 \mathrm{~kg}$ at 6 months and $61.2 \mathrm{~kg}$ at 12 months. Mean BMI fell to $33 \mathrm{~kg} / \mathrm{m}^{2}$ at 6 months. There were two early complications (2\%), a bleeding of access port (conservative therapy) and a gastric perforation (a case of rebanding), that required reoperation (1\%). Late complications $(2 \%)$ consisted of a port dislocation and an erosion (remotion at six months). There were no mortality.

Discussion: These data suggested that throughout the learning curve LAGB can be accomplished with acceptable complication rates, comparable to published outcomes. Duration of surgery improved with experience. Review of complications helps to minimize the occurrence of similar subsequent complications. Despite an advanced laparoscopic experience, a definite learning curve associated with the LAGB must be attained, expecially by young surgeons.

Abstracts

\section{9}

\section{Prosthetic Repair of Left Diaphragmatic Agenesis in Elderly Patient: Report of a Case}

F. Moccia, D. Cuttitta, V. Trapani, L. Fei

Unit of General and Gastrointestinal Surgery-Second

University of Naples, School of Medicine

Introduction: A 71 year-old man affected by left hemidiaphragm agenesis late developed severe constipation and occasional episodes of bowel obstruction. At left subcostal laparotomy, the stomach, the transverse colon, the splenic flexure, and the spleen were located in the left emithorax.

Methods: Repair was performed with a $2 \mathrm{~mm}$-thick expanded polytetrafluoroethylene $\left(\right.$ Gore-Tex $\left.{ }^{\circledR}\right)$ secured in place circumferentially such as a new diaphragmatic dome.

Results: No early major complications and no recurrence at 26 months follow-up has been observed.

Discussion: To our knowledge, this is the oldest treated patient with a true hemidiaphragmatic agenesis and this is the eighth case reported in the literature. The use of the ePTFE soft tissue patch, thanks its strength and pliability, allows a good anatomical and functional repair.

130

\section{The Importance of Neodjuvant Combined RT-CT in the Management of Squamous Cell Esophageal Tumors}

D. Cuttitta, V. Trapani, F. Moccia, L. Fei

Unit of General and Gastrointestinal Surgery-Second

University of Naples, School of Medicine

Introduction: A 48 year-old female came to our evaluation for the recent occurence of increasing dysphagia for solid and liquid food with an associated weight loss of $\sim 20 \mathrm{kgs}$. Her previous history was positive for GERD with grade A esophagitis, treated with PPI.

Methods: She underwent EGDS that revealed the presence of an esophageal stenosis in the lower third of the esophagus. Ecoendoscopy showed a T2Nx tumor that was charachterized by pathological evaluation as a moderately differentiated squamous cell tumor. No distant metastasis at the total body CT-scan were found. The patient was referred to combined neoadjuvant RadioChemiotherapy (RT-CT) according to the following schedule: $1.8 \mathrm{~Gy}$ for 6 weeks (total amount: $46 \mathrm{~Gy})+$ Cisplatinum $\left(35 \mathrm{mg} / \mathrm{m}^{2}\right.$ / week) + Paclitaxel $\left(45 \mathrm{mg} / \mathrm{m}^{2} /\right.$ week $)$.

Results: A new instrumental evaluation, 4 weeks after the end of neoadjuvant treatment, showed the disappearance of the stenosis at the EGDS; ecoendoscopy showed remarkable size reduction of the lesion that was defined as TxNx. The patient was then referred to surgery: through a right thoracotomy and bi-subcostal incision an Iwor-Lewis esophagectomy and two-field lymphnode dissection with an intrathoracic esophago-gastroplasty was carried out. No postoperative complication was observed. Gastrografin X-ray showed no fistulas and a regular transit through the thoracic anastomosis. 
Pathologic evaluation showed a R0 sesection revealing a Tumor Regression Grade (TRG) 2 according to Mandard's classification with rare cancer cells found in the specimen. At 8 months follow-up the patient is free from disease and in good health.

Discussion: We strongly suggest the employement of neodjuvant combined RT-CT in the management of squamous cell esophageal tumors.

\section{1}

\section{Comparison Between Heavyweigh and Partially Absorbable Lightweight Mesches in Incisional Hernia Repair}

\author{
V. Trapani, F. Moccia, D. Cuttitta, L. Fei \\ Unit of General and Gastrointestinal Surgery-Second \\ University of Naples, School of Medicine
}

Introduction: The use of prosthetic material in incisional hernia repair has dramatically reduced the incidence of recurrences. The aim of this clinical study was to compare outcome (in terms of complications and chronic pain) after incisional hernia repair using a nonabsorbable heavyweigh polypropylene mesh or a partially absorbable lightweight mesh, composed by poliglecaprone-25 and polypropylene.

Methods: Fifthy patients (M: 27; F: 23; mean age $57.05 \pm 7.18$ yrs) were enrolled in a randomized prospective study starting on November 2004 and ended on September 2007 after a 12 months mean follow-up. The Rives-Stoppa technique was utilized in all patients. Twentyfive patients were treated with a large polypropylene mesh(Marlex ${ }^{\circledR}$ group) and twentyfive with a large partially absorbable composite mesh (Ultrapro ${ }^{\circledR}$ group).

Results: The results in terms of hospitalization, time to remove the drainage, complications, and chronic persisting pain suggest a lower VAS score in the Ultrapro ${ }^{\circledR}$ group, with no significative differences in terms of complications.

Discussion: The reduction of the inflammatory response seems to be related to a lower incidence of chronic pain as observed in some clinical trials assessing the impact of lightweight meshes in groin hernia repair; more recently the same outcome has been highlighted in a study carried out the incisional hernia repair with heavyweight vs. lightweight polypropylene meshes. In our experience the reduction of pain was more marked in the partially absorbable group as compared to non-absorbable group. In our opinion Ultrapro ${ }^{\circledR}$ mesh provided a similar operative handling compared to a heavy-weight mesh which could represent a balanced compromise between the need of an easier mesh implantation and the reduction of implanted mass.

\section{2}

\section{Non-Anatomical Liver Resections in the Management of Colorectal Metastases: The Role of Surgical Margin Status}

\author{
A. Vecchi, S. De Luca, N. Cautero, F. Di Francesco, \\ P. Garelli, D. Nicolini, G. Martorelli, P. Vincenzi, A. Risaliti \\ Chirurgia Epatobiliopancreatica e dei Trapianti di Fegato, \\ Rene e Pancreas, Azienda Ospedaliero-Universitaria \\ 'Ospedali Riuniti', Ancona
}

Introduction: Liver metastasis represents the major cause of death for patients who have been treated for colorectal tumor. The surgical option can offer long-term survival and resection should be considered when metastases can be totally resected and peroperative examination to exclude occurrence of extrahepatic tumor. In Hepatobiliary Surgical Unit non-anatomical liver resections have become more common in the management of colorectal liver metastases. The aim of this study was to examine the relationship between surgical margin status and site of recurrence.

Methods: Between March 2005-July 2007, 92 patients underwent liver resection for cancer; 46 patients for colorectal metastases had 65 liver resections. Data (demographics, operative data, pathological margin status, site of recurrence and long term survival) were collected prospectively and analysed.

Results: A total of 18 Patients underwent non-anatomical resection, 22 Patients had an anatomical resection, and 6 Patients had a combined procedure. On pathological analysis, resection margins were positive in 5 Patients, negative by $1-9 \mathrm{~mm}$ in 20 , and clear by more than $9 \mathrm{~mm}$ in 21 Patients. At a median follow-up of 12 months, 9 of 45 patients (one patient died at 7th day p.o. due heart attack) developed a recurrence ( 2 lung and 6 liver metastases) but only one recurred at the surgical margin (2nd resection was performed with 5 months follow-up).

Discussion: Non-anatomical resection can be performed with lower rates of surgical morbity and mortality than anatomical resection. Recurrence at the surgical margin was not significantly related to the size of the margin.

\section{3 \\ Intra-Operative Radiation Therapy in Pancreas and Rectal Cancer}

\author{
M. Veroux ${ }^{2}$, P. Fiamingo ${ }^{1}$, B. Zani ${ }^{1}$, P. Valduga ${ }^{1}$, R. Famà', \\ C. Eccher ${ }^{1}$ \\ ${ }^{1}$ General Surgery II Department, S. Chiara Hospital, \\ Trento, Italy; ${ }^{2}$ Department of Surgery, Transplantation and \\ Advanced Technologies - Organ Transplant Unit - \\ University Hospital of Catania
}

Introduction: Intraoperative radiation therapy (IORT) is a cancer treatment modality that allows delivering a single high dose of radiation during a surgical procedure in an unresected tumor, or in a tumor bed area while mobile uninvolved normal tissues are surgically displaced from the radiation beam. 
Methods: IORT is delivered by high dose rate brachytherapy devices (X-rays) or linear accelerators (high energy electrons). Our experience consists in 45 cases of pancreatic cancer treated with surgery followed by IORT (15-30 Gy; 9-12 MeV). In some cases, EBRT plus adjuvant chemotherapy ( $5 \mathrm{FU}$ ) were used. Other 51 patients with locally advanced (36) or recurrent (15) colorectal cancer underwent surgery and IORT (10 to $30 \mathrm{~Gy}$ ). A macroscopically complete removal of the tumor was obtained in 36 patients. In some cases, EBRT (n. 43) and/or systemic chemotherapy (5 FU) (n. 12) was administred.

Results: A global survival after 5 years of $25 \%$ was obtained, among the patients with pancreatic cancer - the main cause of death, in the meanwhile, being due to systematic progression. At 5 years, actuarial probability of cause-specific survival among patients with colo-rectal cancer was $35.3 \%$, of disease-free survival $40.4 \%$ and of loco-regional control 53.3\%.

Discussion: In conclusion, results show that multimodal treatment, including IORT in primary high risk and locally advanced cancer, as well as in the recurrent one, can improve local control of the disease. IORT is a safe and tolerable therapeutic modality, not increasing morbidity or mortality.

\section{4 \\ Impalement Injuries. A Report OH Three Cases}

\author{
A. Vindigni', S. Mazzei', F. Fausto', S.A. Villari', C. Linard', \\ M.A. Gioffrè-Florio ${ }^{1}$
}

'Division of General Surgery, University of Messina, Department of Human Pathology, Messina, Italy; ${ }^{2}$ Service de Anesthèsie et Rèanimation, Centre Hospitalier

Règional, Orlèans, France

Introduction: Impalement injuries are rare and have a large range of variations. Due to their position and extension, they have to be differentiated. The configuration of the offending object, the force and direction of its penetration, and the resting point of its tip dictates the nature and extent of the injuries. These are more injuries in the perineal region than anywhere else. The aim of this study was analyse the results of the treatment for perineal injuries in 3 patients. The method of surgical treatment is recommended in perineal injuries.

Methods: This report is based upon 3 patients whit severe impalement injuries. The causes of these lesions were: accidental anorectal trauma1, accidental rectal trauma 2.

Results: Only 2 patients were operated because the third patient died before surgical treatment. The surgical treatment consisted of the suture of wounds on the perineal way, drainage, antibiotherapy in a case; a colostomy was required in another case due to the complexity of the lesions.

Discussion: Mortality depends on the accompanying injuries and concerns only patients with severe perineal injuries. Severity of the trauma, correct diagnosis of concomitant injuries, the correct usage of antibiotics with a temporary colostomy are key factors for a favourable outcome.

\section{5}

\section{Laparoscopic Gastrectomy: D2 Iynphadenectomy}

\section{S. Grassia, S. D'Angelo, E. Russo, M.G. Ciolli, M. Zuccaro,} P. Lombari

Università degli Studi 'Federico II' di Napoli-II Policlinico, Dipartimento di Scienze Chirurgiche Anestesiologiche Rianimatorie e dell'Emergenza, Direttore Prof. A. Renda

Introduction: Minivasive technique actually is gold standard for colecistectomy. New use of ultrasound based instruments or stapler laparoscopy technology let use of minivasive technique for most several pathology status (benign or malignant pathology). Mini invasive treatment for gastric cancer have not univocal surgical strategy. First lap-gastrectomy for benign disease was perform in 1992. Evidence in cancer gastric demonstrated which was necessary modulated surgical strategy on histology type, size, anatomical position, viscera infiltrating.

Discussion: In recent past news approach rise near laparotomy: the laparoscopy and the hand assisted technique. In this surgery, and particularly laparoscopic technique, the linfoadenectomy is a really important problem, its morbidity and mortality which are high too. Two preliminary randomized study demonstrate useful of restrict at D2 linfoadenectomy for reduce morbidity and mortality. Actually, although knowledge of sovramesocolic region anatomy is indispensable, use of laparoscopy in gastric surgery is feasible and combine the advantage of mininvasive technique whit the 'dictat' of open surgery whit its same survival result. However the long learning curve relegated this procedure at centres whit high-volume perform of gastric surgery.

\section{6}

Molecoular Diagnosis and Colorectal surgery

S. D’Angelo, M. Zuccaro, G. Tedesco, G. Rizzo, E. Russo, N. Carlomagno

Dip. Di Scienze Chirurgiche, Anestesiologiche-

Rianimatorie e Dell'Emergenze, Dir.: Prof. Andrea Renda, Università degli Studi di Napoli, Federico II

Introduction: Complete definition of human genomic is, actually, a realty. The colorectal disease genetic's based like FAP, Colorectal hereditary cancer, HNPCC and IBD syndrome will be better studied and maybe found a medical/genomic care. At these days identify the genetic mutation linked at this condition permit to change surgical strategy.

Methods: In our experience identification of gene mutation in FAP and HNPCC ( $1 \%$ and 5-15\% of cll colorectal hereditary cancer) had changed the diagnosis therapeutic approach. Mutation in loci of APC are implicated in structural modify of this protein, its study on the repercussion on the clinical expression and choice of surgical treatment is really important and actually.

Results: In the surgical proceeding the use of proctocolectomy whit a ileo anal anastomoses or colectomy whit rectal preservation result in ileo-rectal anastomoses is choice based on type of mutation

Eur Surg Res 2008;40:61-183 
and so the time to go on surgical treatment using the family division in late and early onset.

Discussion: The mean of prophylactic surgery is unclear. For the IBD actually, we have only observation study on familiarity and any mutation-gene linked at these pathology. The new technology for screening and follow up to apply at each high risk population to permit a best control of these disease.

\section{7}

Mesorectal Excision: Post-Operation Valuation

M. Zuccaro, S. D'Angelo, M. Clemente, G. Tedesco,

M.G. Ciolli, N. Carlomagno e P. Lombari

Università degli Studi 'Federico II' di Napoli-II Policlinico, Dipartimento di Scienze Chirurgiche Anestesiologiche Rianimatorie e dell'Emergenza, Direttore Prof. A. Renda

Introduction: Surgery of rectal neoplasia is really most binding surgical technique. In particular the TME is a basic surgical time for respect some oncologist criteria: integrity of mecorectal fascia, radial margin, dissection in holy plane, no effect Andemberg, for this target is indispensable confront whit pathology specialist.

Discussion: The surgeon variable is the volume of mesorectal excision. Under $12 \mathrm{TME} /$ year perform the locally recurrence is $10 \%$, the surgeon which perform up $12 \mathrm{TME} /$ year have a locally recurrence ratio 4\%. Macroscopic valuation of the speciment in the most part of case follow the Quirke criteria. Its divide into three class the excision: complete, almost complete and incomplete. Prognostic valuation is based on the lymphnode invasion and they valuation in function of anatomical position (high or medium or inferior rectum), but in every way the examination speciment must have 12 lymphonode. More technique useful for found lymphnode until $1 \mathrm{~mm}$, sentinel lymphonode technique is used but has very high false negative ratio. Pathologist examination is very important prognostic factor but also permit a auto - valuation procedure in learning curve and value of integrated treatment.

\section{8}

\section{Signet Ring Cell Gastric Cancer: Analysis of Result at Distance}

M. Clemente, S. D’Angelo, M. Zuccaro, G. Spinosa,

S. Spiezia e M. Santangelo

Dip. Di Scienze Chirurgiche, Anestesiologiche-

Rianimatorie e Dell'Emergenze, Dir.: Prof. Andrea Renda, Università degli Studi di Napoli, Federico II

Introduction: Signet ring cell (SRC) gastric carcinoma occurred more frequently in the middle third of the stomach. This type of carcinoma appears to be relatively frequent in woman and young patients and it has a variable prognosis.

Methods: Between 1990 and 2005, 310 patients suffering from gastric cancer were treated surgically at the our department. A total of $34(13.7 \%)$ had a histological diagnosis of SRC carcinoma of stomach.
15 of $34(35.7 \%)$ had a disease in early stage and 26 of $34(64.3 \%)$ had advanced gastric carcinoma. All patients have showed the overall 36-months of $52.6 \%$.

Results: In the our case histories, SRC carcinoma is localized in the lower third of the stomach and appears to be relatively frequent in males.

Discussion: In this study, statistically significant adverse factors are: ipoalbuminemia $(p=0.002)$, tumour size $(p=0.05)$, tumour stage $(\mathrm{p}=0.05)$ and lymph node metastasis $(\mathrm{p}<0.0001)$. In the early SRC gastric cancer, the overall is better than that of patient with non SRC gastric cancer, therefore we can consider the subtotal gastrectomy with accurate limphnodal dissection as gold standard for the treatment of this gastric cancer. On the contrary, the behaviour of the advanced SRC carcinoma of stomach is more aggressive than this of the other types of gastric carcinoma e for this it necessitates a more large limphnodal dissection.

\section{9}

\section{Conservative Surgery of Pancreas Rare Tumour}

S. Spiezia, M. Clemente, G. Spinosa, M. Zuccaro, S. D'Angelo e M. Santangelo

Dip. Di Scienze Chirurgiche, AnestesiologicheRianimatorie e Dell'Emergenze, Dir.: Prof. Andrea Renda, Università degli Studi di Napoli, Federico ॥

Introduction: Pancreas's solid-cystic papillary are very rare tumours. This tumour has a preference for young women and appears as a mass of large size, clinically silent or with unspecific symptoms. Tumour is more frequently localized in the pancreas and is rarely a metastatic disease. The prognosis is very good, with long term survival also in patients with metastases or unresectable tumours. Surgery is the elective treatment.

Methods: A 17-year-old woman was admitted to our institute because of abdominal pain and 15-days long vomiting. At physical examination, a palpable mass in the upper abdomen was found. Ultrasound (US) revealed a large mass in the upper abdomen with non homogeneous echogenicity. A computer tomography (CT) scan showed a round encapsulate mass of pancreas. Intraoperatively, neoplasm originated from pancreas body with infiltration stomach and duodenum. A central pancreatectomy with anastomosis between pancreatic head and Roux-ansa was performed. Caudal stump of pancreas was an-block sutured.

Results: The postoperative course was uneventful and the patient is alive and disease-free at a follow-up of 48 months. 


\section{Gynecological Surgery}

\section{0}

\section{Conservative Treatment for Severe Pelvic Organ Prolapse}

\author{
D. De Vita ${ }^{1}$, F. Araco ${ }^{2}$, G. Auriemma ${ }^{1}$, D. Lullo', E. Piccione ${ }^{2}$ \\ ${ }^{1}$ Section of Obstetrics and Gynecology, S. Francesco \\ D'Assisi, Oliveto Citra, SA, Italy; ${ }^{2}$ Section of Gynecology \\ and Obstetrics, Department of Surgery, School of \\ Medicine, 'Tor Vergata' University Hospital of Rome, Viale \\ Oxford 81, 00133 Rome, Italy
}

Introduction: Genital prolapse is a common problem, with a cumulative risk of undergoing anti-incontinence and/or genital prolapse surgery by age 80 years of $11.1 \%$. A lot of advantage vaginal surgical techniques using prostheses have been proposed in the last years, with some intraoperative and postoperative complications. Vaginal erosion has been a possible complication of vaginal surgical techniques using polypropilene mesh.

Methods: New techniques, enveloped to Centre for Pelvic Recostructive Surgery at Sydney Adventist Hospital, have been used to Department of Obstetric and Gynaecology 'S. Francesco D'Assisi', Oliveto Citra, Salerno and to Gynaecological Departments of University 'Tor Vergata' in Rome from September 2004 to November 2006 on 80 patients. Polypropylene prostheses (Gynemesh-Soft PS, $10 \times 15 \mathrm{~cm}-$ GyneMesh, Gynecare Ethicon) were used to reconstruct the pubo-cervix or the recto-vaginal fascia. We performed an anteriorcentral vaginal reconstruction in $35(43.8 \%)$ patients, central-posterior in $25(31 \%)$ and total reconstruction in $20(25 \%)$.

Results: The mean follow-up was 18 months (range 4-26). The severe pelvic prolapse, evaluated with the POP-Q System, was completely treated in all the patients and no recurrences were observed. We recorded 3 vaginal erosion and one patient complained of a postoperative dispareunia.

Discussion: This pilot study suggests that our technique is safe and effective and can efficiently repair the pelvic organs prolapse without undergoing hysterectomy and with a low rate of vaginal erosions.

141

\section{Epidemiologic Study on the Incidence of Social, Territorial and Personal Data and Delivery Modality on Urinary Incontinence and Genital Prolapse in Salerno'S Popolation}

\author{
D. De Vita', M.C. Muccione', F. Palma², R. Rinaldi², \\ M. Calenda ${ }^{3}$, D. Lullo', G. Auriemma ${ }^{1}$ \\ ${ }^{2}$ Section of Obstetrics and Gynecology, S. Francesco \\ D'Assisi, Oliveto Citra, SA, Italy
}

Introduction: Genital prolapse is a common problem, with a cumulative risk of undergoing anti-incontinence and/or genital prolapse surgery by age 80 years of $11.1 \%$. The aim of this retrospective epidemiologic study has been to evaluate incidence and risk factors of urinary incontinence and genital prolapse in the population that refers to uro-gynaecological department of Olivetro Citra Hospital.

Methods: We have enlisted 330 patients with stress urinary incontinence and genital prolapse. The study performed by the compilation of 330 uro-gynaecological charts. The compilation of charts included anamnestic history and social, territorial and personal data. Particularity, the study analysed: age, schooling, parity, delivery's modality, Body Mass Index and previous gynaecological operations.

Results and Discussion: The results obtained were: an important role played by social and demographic data of the population studied. The risk factors of stress urinary incontinence and genital prolapse have been: age, profession (agriculture), Body Mass Index (B. M. I.) and previous gynaecological operations (isterectomy), delivery's modality (parity, fetal weight) and menopause.

\section{2 \\ IUS and Obstruced Defecation Syndrome:Our Experience on a Multidisciplinary Treatment}

G. Rota ${ }^{1}$, D. Parmeggiani², M. Perrotta ${ }^{2}$

${ }^{1}$ Division of Obstetrics and Ginecology, ${ }^{2}$ Division of General Surgery, P.O. ' S. Giovanni di Dio' -Frattaminore, Napoli, ASL Na 3

Introduction: Stress urinary incontinence with urethral hypermobility is often associated to a cystocele, with obstructed defecation syndrome

Methods: Since October 2003 to January 2005, 6 female pts (a.a.51 ys) with obstructed defecation and stress urinary incontinence underwent to surgical correction. In our study we've not included cystocele biger then 1st degree with syndrome of obstructed defecation. The first surgical approach was a TOT (Trans Obturator Tape), in the same time the second step was a STARR (Stapled trans anal rectal resection).

Results: Up to 6 until 12 months follow up all pts were not wet after the stress test and without instability and/or hypomobility at the urodinamic tests. All the pts had not problem of stipsis.

Conclusion: We suggest the choice of a multidisciplinary treatment in the management of Stress Urinary Incontinence associated to Obstructed Defecation syndrome. 


\section{Laparoscopic Surgery}

143

\section{Safety of Laparoscopic Cholecistectomy Performed by Residents}

\author{
L. Bencini, L. Moraldi, M. Bernini, E. Miranda, F. Martini, \\ R. Moretti \\ 3rd Division of General Surgery - Careggi, Main Florence \\ University and District Hospital, Viale Morgagni 85 - \\ 50134 Florence, Italy
}

Introduction: Laparoscopic cholecistectomy is becoming widely performed by Italian surgical residents, but specific outcomes have been poorly investigated, as compared to those carried out by experienced surgeons.

Methods: Data from 188 consecutive, unselected patients who underwent LC performed by residents between 2000 and 2006 at our institution were collected. An equal number of LC performed over the same period by experienced surgeons were randomly extracted from the database. Demographics, periopeoperative parameters and complications were matched among the two groups.

Results: Age, gender, ASA score, length of stay, proportion of urgent operation and cholecystitis were all well matched in the two groups. No major complications were collected in both series. The number of IOC, rendez-vous, drainages, and minor complications were also equal. Nevertheless, the overall conversion rate and the mean difficulty score $(0-20)$ were lower among residents ( 1 vs. 5\%, $p=0.03$ and 6.65 vs. $7.32, p=0.04$ respectively). On the other hand, the mean duration of surgery was significantly longer in the residents group ( 71 vs. 62 minutes, $\mathrm{p}<0.000$ ).

Discussion: Italian residents have fewer possibilities to gain sufficient skills at the end of their residentship, as compared to their foreign colleagues due to social, cultural, political and academic troubles. However, LC is a safe and common procedure even in the hands of Italian trainees, although patients' selection is strictly required. In fact, tthis study found that residents performed less complex operations in more time, as compared to senior surgeons.

\section{4}

\section{A Case Report of Laparoscopic Posterior Rectopexy for Complete Rectal Prolapse (CRP)}

\section{Bertoglio, A. Frigerio, P. De Martini, I. Scandroglio}

Az. Ospedaliera di Busto Arsizio, Tradate

Introduction: Rectopexy is the treatment of choice for CRP. Data from literature show that laparoscopic Wells procedure(LW) would give better short term results than transabdominal approach.

Methods: We report a case of 59 years old man complaining of hematochezia due to CRP. Dynamic videoproctography was performed preoperatively. 3 trocars were inserted and patient placed in trendelenburg position. The peritoneum was incised over top of promontory and dissection proceeded between sacral fascia and fascia recti down to the coccyx. Lateral opening of peritoneum provided wide space for introduction of composite prosthetic mesh (M). Prosthesis was tailored and wrapped around the rectum leaving a defect in the anterior midline and then fixed to posterior sacral midline with titanium staples. Same devices were used to fix lateral margins of M.2PDS continue suture closed peritoneal defect over the M.

Results: Operation time was $96 \mathrm{~min}$. No significant complication was recorded except for minimal subcutaneous emphysema. Bowel movement returned on postoperative day1 while first flatus and return to solid oral intake occurred on pd2. Hospital stay was 4 days and patient experienced alleviation of symptoms at short term follow up of 6 months.

Discussion: LW is a safe and feasible procedure even in elderly and debilitated patient giving good functional results in terms of symptoms relieve and low recurrence rate.

\section{5}

\section{Learning Curve of Laparoscopic Colorectal Resections Perfomed by an under 35 Years Old Surgeon: Does Surgeon's Age Influence Results?

\author{
L. Boni, E. Cassinotti, M. Diurni, G. Dionigi, L. Giavarini, \\ M. Di Giuseppe, F. Cantore \\ Ospedale di Circolo di Varese
}

Introduction: Several studies, including large multi centric randomized trial demonstrated that laparoscopic surgery (LS) is feasible and effective also for the treatment of benign and malignant colorectal disorders. Surgeon's age and experience has been historically regarded as a possible factor influencing the surgical outcome both in open and LS.

Aims: The present study report the results of laparoscopic colorectal resection (LCR) performed over a period of one year by a single less of 35 years old surgeon.

Patients: 11 patients admitted at the Department of Surgery of the University of Insubria (Varese, Italy) undergone elective LCR of different diseases has been retrospectively reviewed. All procedure has been performed by the same surgeon with less of 35 year old, fully trained in open and with extensive experience in laparoscopic surgery.

Results: From June 2005 and June 2007, 172 LCR has been performed for different diseases, including cancer 147, benign polyps 20 , IBD 5. The mean procedure time was $164 \pm 22 \mathrm{~min}$ (raging from $154 \pm 16 \mathrm{~min}$ in case of cancer and $335 \pm 25$ from IBD). There were no intra-operative complications and or mortality, while the postoperative complication rate was $13.5 \%$. The mean post-operative stay was $6.2 \pm 2.2$ days (range 4-16). In case of cancer, the mean number of resected lymph-nodes was $22 \pm 12$.

Conclusions: Results of LCR are not influenced by surgeon's age.

20th National Congress of the Italian Polyspecialist Society of Young Surgeons (IPSYS) 


\section{6}

\section{Laparoscopic Treatment of Recurrent Massive Mesenteric Cyst}

\author{
L. Boni, E. Cassinotti, L. Giavarini, M. Di Giuseppe, \\ F. Cantore \\ Ospedale di Circolo di Varese
}

The video shows a case of a 57 year old, white, non smoking, non alcoholic woman was admitted to the Emergency \& Accident ward, complaining of a sudden onset of sharp abdominal pain together with nausea and vomit associated to increasing of abdominal diameter. Past medical history included surgical removal of the appendix and laparoscopic removal of a small $(3 \times 3 \mathrm{~cm})$ ovarian cyst 12 months before. At examination a large $(15 \times 10 \mathrm{~cm})$, painfull mass was present occupying most of the abdomen, mainly located in the middlelower part. Laparoscopic exploration of the abdomen was carried out. a large cystic mass, attached to the small and large bowel a covered by the greater omentum was found. Laparoscopic aspiration device was placed into the cyst in order to create a larger working space, and almost 2,000 $\mathrm{cc}$ of dense, white fluid (resulted 'sterile' at microbiology) was removed. Marsupialisation of the cyst was then carried out, by removing of most of the cystic wall using computer controlled bipolar device, cauterisation of the remaining wall by argon beam and placing an omental patch fixed by $2 / 0$ absorbable suture. Histopathological examination of the cystic wall confirmed the benign nature of the lesion. The patient's post-operative course was uneventful and she was discharged on day 3 postoperatively.

\section{7}

\section{Learning Curve in Robotic Surgery and the 'Young Surgeon'}

G. Ceccarelli, A. Patriti, A. Bartoli, A. Spaziani, L. Casciola

Divisione Chiruirgia Generale Vascolare e Mininvasiva, Spoleto

Introduction: During the last 10 years, robotic surgical systems in General Surgery have evolved from simple devices (stabilizers, voice-controlled camera) to more complex solutions. The da Vinci ${ }^{\circledR}$ Surgical System is the most advanced robotic device available today in General Surgery.

Methods: We used the da Vinci ${ }^{\circledR}$ System from September 2002 in more than 3 hundred operations. It consists of an ergonomically surgeon's console connected to a cart with three or four interactive robotic arms; articulated (EndoWrist ${ }^{\mathbb{B}}$ ) instruments with 7 degrees of freedom, a high-performance vision system (high resolution 3-D image). The tremor reduction and motion scaling allows more precision and dexterity. This device has FDA clearance for a wide variety of surgical procedures, in different specialities: Cardiac, General Surgery, Urology, Thoracic.

Results: The first operations performed with the da Vinci ${ }^{\circledR}$ System, as in laparoscopy, were simple procedures as cholecystectomy, hiatal hernias treatment. Now it is possible to perform more complex operations as: gastric, intestinal, splenic, pancreatic surgery.
Our robotic series was evaluated retrospectively recording complications, operating time, hospital stay, patient benefits.

Discussion: As in laparoscopic surgery, a specific training, and learning curve are needed; so a dedicated team (surgeons-nursesanesthesiologists) at the beginning of the experience is required. All the components of the 'robotic surgical team' have to turn from operating table to console, to reach experience in every part of the robotic technology. We report our experience about learning curve in Robotic Surgery from young surgeons of the equipe.

\section{8 \\ Diagnostic and Therapeutic laparoscopy in Pelvic Emergencies

G. Ceccarelli, A. Patriti, A. Spaziani, A. Biancafarina,
L. Casciola
Divisione Chiruirgia Generale Vascolare e Mininvasiva,
Spoleto

Introduction: The diagnosis of acute appendicitis in women in the reproductive age is sometimes demanding during preoperative work-up. We have reviewed our experience about diagnostic and therapeutic laparoscopy for acute and chronic pelvic pain. The aim of this retrospective study was to determine the usefulness of laparoscopy in these setting.

Methods: We have studied 290 cases of females in the reproductive age treated with laparoscopic approach during 2000-2006. 236 for acute and 54 for chronic pelvic pain. The mean age of patients was 27 years (range 13-49), The more frequent intraoperative diagnosis was acute appendicitis but $21 \%$ of acute pelvic pain was of gynaecologic origi: pelvic inflammatory disease, complicated ovarian cysts, torsion of adnexal mass, ectopic pregnancy. Causes of chronic pain were: adhesions, endometriosis and in 4 cases of Fitz-HughCurtis syndrome.

Results: The conversion rate of laparoscopic approach was $1.4 \%$ ( 4 cases). A diagnosis was possible during laparoscopy in $98 \%$ of the cases; operative complications were 8,3 requiring reoperation ( 2 for pelvic abscess after acute appendicitis). No mortality was observed, the trocar site complications were the more frequent in this series $(2.1 \%)$.

Discussion: Laparoscopy plays an important rule for the management (diagnosis and treatment) of acute and chronic pelvic pain. An high percentage of suspected acute appendicitis in women in reproductive age, are gynaecologic emergencies. A laparoscopic approach with early diagnosis and conservative management, may prevent complications and preserve fertility. Laparoscopy is safe and effective as laparotomy, but it results in shorter hospital stay and faster recovery. 


\section{9}

\section{Combined Laparoscopic Cholecystectomy and Incisional Hernia Repair}

\author{
E. Coppola Bottazzi, G. Mastrobuoni, M. Di Marino, \\ C. Cirillo, M. Cacciapuoti, M. Persico, S. Masone, G. Aprea
}

University of Naples 'Federico II'; School of Medicine, Department of General and Geriatric Surgery and

Diagnostic and Operative Endoscopy, (Chief: Giovanni

Persico)

We report a case of calculous cholecystitis and incisional hernia (previous hysterectomy) in a 57 years old woman, treated with laparoscopy. We positioned 4 trocar, 2 of $10 \mathrm{~mm}$ in diameter (umbilical optical access, open laparoscopy) and 2 of $5 \mathrm{~mm}$ (French technique). After cholecystectomy, performed without any loss of biliary material, optics was turned towards pelvis and, targeted the abdominal wall defect, filled with omentum and intestinal ansae, we proceeded to accurate unbridling and preparation of the gap. Then we positioned a Dual Mesh $10 \times 15 \mathrm{~cm}$ PTFE prosthesis, fixed with Protrack spirals. After 1 week, 1 month, 6 months and 1 year controls, patient was disease free. Combined operation of cholecystectomy and incisional hernia repair with prosthesis positioning must necessarily be performed without any biliary contamination of the operating field. Laparoscopy allows easier operation performance, with also minimal surgical trauma.

\section{0 \\ Achalasia. Our Experience with the Laparoscopic Approach}

\section{A. Salvucci, C. De Martino, R. Guarino, G. Capuano, M. Milone, M. Musella \\ Naples 'Federico II' Medical School, Department of Surgery and Orthopaedics, General and Emergency Surgery}

Introduction: Authors present their experience of 30 case of achalasia treated with laparoscopic surgery.

Methods: From January 2002 to 2007, 30 patients with achalasia were treated by a laparoscopic approach. Females were 20 and males were 10. Median age was 31.2. All of patients were studied with endoscopy and oesophagus manometry for the classification of achalasia. They were treated by Heller myotomy and fundoplication.

Results: Pre-operative weight loss was $9 \mathrm{~kg}$ (range 3-16) with a mean duration of symptoms of 29 months (12-72). All patients had a laparoscopic Heller myotomy. Thirty percent received a partial anterior fundoplication according to Dor whereas the remaining seventy percent (21 of 30 ) had a complete fundoplication according to Nissen-Rossetti. The mean operative blood loss was $70 \mathrm{ml}(30-150)$. All patients beared a NG tube for 3 days, and an X-ray contrast swallow was performed thereafter. Oral feeding started in case of negative $\mathrm{X}$-ray control. One patient developed an oesophageal fistula. Followup ranged up to 4 years. Postoperatively, symptoms of dysphagia (to both solids and liquids), regurgitation, and cough were significantly reduced in all patients.
Conclusion: Laparoscopic cardiomyotomy with anterior partial or complete fundoplication achieves excellent symptomatic relief for patients with achalasia, and it can be performed with minimal morbidity.

\section{1 \\ 'One-Stage' Laparoscopic Treatment in Cholecystocholedocholitiasis}

\author{
P. Della Rocca, A. Trombetti, D. Cuccurullo, F. Pirozzi, \\ F. Corcione
}

Chirurgia Generale, Ospedale Monaldi, Napoli

Introduction: The laparoscopic cholecystectomy is the treatment of choice for the management of cholelitiasis. For the choledocholitiasis several therapeutic options exist. The advent of endoscopic techniques changed surgery in many ways. Several studies have shown the efficacy of the combined endoscopic-laparoscopic technique in 'one stage' procedure for treatment of gallbladder and bile duct stones.

Methods: From January 1999 to December 2007, we made 3,720 laparoscopic cholecystectomies with 305 cholecystocholedocholitiasis confirmed by intraoperative cholangiography. In 49 cases we performed the sequential treatment (endoscopic retrograde cholangiopancreatography followed by laparoscopic cholecistectomy), in cases with patient-related risk factors for anestesya, older patients and patients with doubt diagnosis. In 49 cases we made 'one stage' laparoscopy ( 30 choledochotomy and 19 choledochoscopy). In 2 cases we performed a laparoscopic transduodenal papillotomy in patients with subtotal gastrectomy. The Rendez-vous technique, performed in 205 patients, consists of laparoscopic cholecystectomy standards with intraoperative cholangiography followed by endoscopic sphincterotomy through a guidewire inserted by the transcystic duct. Thus method allows elimination ductal stones with faster and easier cannulation of Vater papilla reducing papilla edema and pancreatic trauma.

Results: In 3 cases the conversion to open procedure was necessary. there was complication in $8.1 \%$ of the cases ( 4 bleedings, 3 coleperitoneus, 2 recurrence, 14 postERCP pancreatitis, 2 mortality). The mean operative time was 150 minutes. The mean hospital stay was 6 days.

Discussion: We believe that the combined laparoscopic-endoscopic approach should be considered the treatment of choice in cholecysthocholedocholithiasis. The advantages of this technique are a shorter hospital stay, a reduction in costs, the higher compliance of the patient, a reduction of postERCP pancreatitis and the minimal incidence of recurrences but required an high organization of the operating room and a strict collaboration between surgeon and endoscopic. 


\section{2}

\section{Laparoscopic Colorectal Surgery by Radiofrequency Dissector as Solo Instrument for Dissection, Haemostasis, Vessel Sealing and Cutting. Three Years Experience on 100 Cases}

\section{R. Dell'Avanzato, M. Carlini \\ U.O.C. Chirurgia Generale, Toracica e Addominale - Ospedale S.Eugenio}

Introduction: The video shows a laparoscopic rectosigmoid resection for a T2-adenocarcinoma of the rectosigmoid junction.

Methods: The operation is performed by the radiofrequency dissector Ligasure ${ }^{\mathrm{TM}}$ Atlas (TycoHealthcare) solo, without scissors, ties, mono-bipolar coagulators and clips. The operation begins with the isolation, closure and section of the inferior mesenteric artery and vein. The laterocolic dissection, the left colonic flexure mobilization, the mesocolon section are easily performed. The rectum is transected by a linear endoscopic stapler and cutter (EndoGIA60). A colorectal Knight-Griffen anastomosis is performed with a transanal circular stapler (CEEA $28 \mathrm{~mm}$ ). In our experience 100 patients between 2004-2006, affected by colorectal disease were candidate to laparoscopic resection.

Results: 18 procedures were converted to open surgery due to: T4 tumours (15), ureteral injury (1), lacked identification of the lesion (1), severe obesity (1). The remaining 82 procedures were laparoscopically completed. The blood loss was null. Complications were observed in 8 cases: fistulas (4), wound infections (3), jejunal haemorrhage (1).

Discussion: The video shows the feasibility, effectiveness and reproducibility of a left laparoscopic colectomy performed with the radiofrequency dissector Ligasure ${ }^{\mathrm{TM}}$ Atlas solo. The technique is the standard procedure in laparoscopic colorectal resections.

\section{3}

\section{Results in Laparoscopic Approach in Rectal Cancer Surgery: Postoperative Outcome and Short-Term Survival}

\author{
N. Furlan', S. Bona1, U. Elmore', P.P. Bianchi', M. Rottoli ${ }^{2}$, \\ U. Fumagalli', M. Montorsi ${ }^{2}$, R. Rosati ${ }^{1}$ \\ ${ }^{1}$ Departments of General, and ${ }^{2}$ General \& Minimally \\ Invasive Surgery, University of Milan, Istituto Clinico \\ Humanitas IRCCS, Rozzano, Milano, Italy
}

Introduction: Minimally invasive surgery for rectal cancer is being evaluated worldwide. We analyzed short-term and oncologic results in our series of laparoscopic rectal resections for cancer.

Methods: One-hundred-forty-one patients with rectal cancer were operated on with laparoscopic approach at our Institution. Patients with locally advanced disease (invasion of adjacent structures and/or external anal sphincter), distant metastases, previous colonic surgery or general contraindications to laparoscopy were excluded from the study. Survival was calculated by the Kaplan-Meier method.
Results: A totally laparoscopic procedure was completed in 116 patients (113 sphincter-saving procedures, 2 Miles operations and 1 laparoscopic Hartmann procedure). Conversion rate was $17.7 \%(25 / 141)$. Mean duration of laparoscopic procedures was $285 \mathrm{~min}$ (range 135-437). Major postoperative complications occurred in $18.4 \%$ patients and anastomotic leak rate was $11.3 \%$. No postoperative deaths occurred. Pathologic examination showed a mean of 18.3 lymphnodes on specimen (range 1-58) and mean distance of distal margin from tumor was $2.57 \mathrm{~cm}$ (range 0.5-10). In 2 case microscopic invasion of the distal margin was detected. Mean postoperative hospital stay was 8 days (range 4-43). After a mean follow-up of 35.4 months survival rate is $91.5 \%$. Local recurrence was detected in 4 patients $(2.8 \%)$. No port site metastases were reported. Actuarial five-year and disease-free survival rates are $82.1 \%$ and $80.2 \%$ respectively.

Conclusions: Laparoscopic rectal surgery is safe and oncologically correct. Nevertheless technical difficulties still account for a high conversion rate and long operative time. Sound experience in colorectal and minimally invasive techniques are required when approaching laparoscopic rectal surgery.

\section{4}

\section{Laparoscopic Treatment of Gastroesophageal Reflux Disease}

\section{F. Stanzione, G. Conzo, M.G. Esposito, U. Brancaccio, C. Della Pietra, A. Palazzo, S. Celsi, A. Livrea}

IV Divisione di Chirurgia Generale e di Endocrinochirurgia - Seconda Università degli Studi di Napoli

Introduction: Laparoscopic (VL) fundoplication represents a 'gold standard' in the treatment of gastroesophageal reflux disease (GERD). The indications for surgical treatment were well established by the Consensus Conference of European Association for Endoscopic Surgery (EAES-2004) [failure of long term medical therapy, intractable or recurrent symptoms, complications, large sliding or paraesophageal hiatal hernia].

Materials and Methods: Since January 2003 to December 2006, 50 patients (pts) [21 F; $29 \mathrm{M}$, average age $42.5 \mathrm{ys}$ ], underwent to laparoscopic Nissen fundoplication ('Floppy Nissen') for GERD: in 16 pts hiatal hernia was associated. In 3 young pts the indication to surgical treatment was given in absence of response to PPI full dosage medical therapy. Surgical treatment consisted, under endoscopic guide, in 'open' laparoscopy with 5 trocars ( 3 of $10 \mathrm{~mm} ø$ and 2 of $5 \mathrm{~mm} \varnothing)$ in a $30^{\circ}$ reversed-Trendelemburg position by gastroesophageal junction dissection, mediastinic esophageal mobilization, crural repair performing a 'Floppy Nissen'. The short gastric vessels were never dissected. In II p.o. the patients were discharged after an esophageal gastrographin Xray control.

Results: No complications were observed. In any case a variable dysphagia was present.

Conclusions: VL approach to the GERD represents a 'gold standard' for the recognized and typical advantages of minimally invasive surgery and probably allows a better dissection and identification of the anatomic structures (vagus, pleura). $360^{\circ}$ fundoplication shows a better control of GERD and a lesser incidence of recurrence than partial wraps. Intraoperative endoscopy is helpful for the control 
of visceral integrity and the calibration of the wrap. Long term follow up is necessary for the evaluation of results.

\section{5}

\section{Fast Track Surgery: A Modified Protocol Applied in 80 Consecutive Laparoscopic Colonic Resections in a Rural Hospital of the Tuscany Public Health-System}

\author{
F. Feroci, M. Scatizzi, M. De Prizio, K. Kroning \\ U.O. Chirurgia II, Ospedale S. Maria alla Gruccia (ASL \\ 8-Arezzo)
}

Background: The experience of multimodal management of patients undergoing colorectal surgery applied by Prof. H. Kehlet has made it possible to decrease non-surgical postoperative complications and allows for a quicker release from hospital. Colorectal laparoscopic surgery in comparison to open colorectal laparoscopic surgery without treatment 'Fast track,' has better results in regards to pain, respiratory performance and reduction of postoperative hospitalization. We started our protocol on the application of these two procedures in patients submitted to colorectal laparoscopic surgery in a peripheral hospital of the SST (Tuscan Sanitary Service) in Italy.

Methods: From November 2004 to November 2006, 80 patients had laparoscopic right or left colic resection or laparoscopic low anterior resection (AR) and we gave them a modified multimodal fast track program.

Results: The mean postoperative hospitalization was 4.7 days for laparoscopic left hemicolectomy and laparoscopic anterior resection group, and 5.1 days for the right hemicolectomy group. The percentage of observed complications was $6.4 \%$, one patient was converted to open procedure $(0.8 \%)$; two patients $(1.6 \%)$ were readmitted in the hospital within 30 days. No mortality was observed.

Conclusions: The results of the two procedures were feasible in the observed context, and effective in the reduction of almost $50 \%$ of the mean postoperative hospitalization compared to patients in Europe who had the same kind of operation without the application of these two procedures.

\section{6}

\section{Laparoscopic Antireflux Surgery of Large Hiatal Hernia with Prosthetic Reinforcement of Cruroplasty}

\author{
R. Lionetti, R. Brunaccino, D. Fico, P. Di Silverio, \\ D. Pelaggi, S. Capasso, E. Di Frattta, S. Dilillo, G.P. Ferulano \\ Chirurgia Generale, Policlinico Federico II, Napoli
}

Introduction: Several studies have shown that in large Hiatal Hernias $(\mathrm{HH})$ recurrence with wrap migration in the chest is a common complication after Laparoscopic Nissen Fundoplication (LNF). The use of an adequately stitched mesh should decrease the rate of recurrence in these cases. We present the results of 7 cases treated by LNF and coated prosthesis, at one year follow-up.
Methods: 7 patients ( 5 men, 2 women, mean age 56.4 months) with a type III HH and a hiatus size $\geq 6 \mathrm{~cm}$ underwent a floppy LNF with posterior cruroplasty and coated prosthetic mesh fixed to the crura by Q-ring fixation system $\left(\mathrm{SALUTE}^{\circledR}\right)$. Surgical procedure included: dissection of oesophageal hiatus, sac excision and dissection of the abdominal oesophagus to obtain a physiological length.

Results: Mean operating time was $127 \mathrm{~min}$, postoperative recover was uneventful, mean hospitalisation length was 4 days. At one year manometric control we present the mean results of the following parameters: LES pressure: $21 \mathrm{~mm} / \mathrm{Hg}$; LES length: $3.5 \mathrm{~cm}$; LES relaxation: $93.8 \%$; and intra-abdominal LES length $2.3 \mathrm{~cm}$. The amplitudes of contractions were normal and the rates of simultaneous waves and interrupted waves were not significant.

Discussion: LNF with prosthetic crural closure in large size $\mathrm{HH}$ ( $\geq 5 \mathrm{~cm}$ ) can be performed quite safely with good surgical and functional results using a protected mesh adequately fixed.

\section{7}

\section{Laparoscopic vs. Open Surgery for Extraperitoneal Rectal Cancer: Preliminary Results}

R. Brunaccino, R. Lionetti, D. Fico, D. Pelaggi, S. Capasso, E. Napolitano, M. D'Ambra, S. Dilillo, G.P. Ferulano

Chirurgia Generale, Policlinico Federico II, Napoli

Introduction: Long-term results provide level I of evidence that laparoscopic surgery (LS) for colon cancer is oncologically adequate although in terms of rectal cancer (RC) no level I evidence is available.

Methods: A series of unselected consecutive 10 patients with RC, who undergo LS in our Department during 2 years (Jan. 2006-Dec. 2007), have been included in this study. Patients with T1N0 tumours and emergency cases are excluded from the study. All T3-T4 or any TN1 undergo preoperative radiotherapy (RT) and, in selected cases chemotherapy. The surgical procedure is performed 6 weeks after RT. The control group is a series of consecutive 13 patients with RC operated during the previous 2 years.

Results: The following parameters were compared between the two groups in short term outcomes: Perioperative parameters (conversion rate, blood transfusions, anastomotic dehiscence, wound infection, postoperative pain, hospital stay); Oncologic adequacy on resected specimen (length and margins, lymph nodes dissection, prevention of intraoperative tumour cell dissemination, quality of mesorectal excision); Functional preservation (autonomic pelvic nerve, anal sphincter preservation). Laparoscopic approach improves perioperative parameters, respecting radicality and phisiological functions.

Conclusions: LS in the treatment of lower RC is safe and effective. A longer follow up is necessary for long term outcome. 


\section{8}

\section{Laparoscopic Lower Resection of the} Rectum: Our Experience with Curved Stapler

F.S. Mari, L. Masoni, A. Brescia, M. Gasparrini, A. Milillo, F. Favi, L. Angelini

UOC Chirurgia B, Responsabile Prof. Licinio Angelini, UOS Chirurgia dell'ano retto e pavimento pelvico, Responsabile Prof. Luigi Masoni, Azienda Ospedaliera Sant'Andrea Universita' La Sapienza di Roma - II Facolta' di Medicina e Chirurgia

Introduction: Laparoscopic resection of the rectum (LRR) is today routinely perfomed in many Center. The most difficult part of the lap procedure is represented by sectioning of the lower rectum especially in the presence of large tumors, narrow male pelvis, and often requires conversion by a Pfannestiel incision and manual completion of dissection. We routinely perform LRR by a three to four trocar technique and use for lower rectum section the Contour stapler (CS). Differently from Endo-GIA staplers, this device closes the rectum quite always in a single shot.

Methods: We have experimented an original technique based upon the insertion of the CS through a Lapdisc ${ }^{\circledR}$. A 6-7 cm suprapubic midline incision allows positioning of the Lapdisc. Gas leak along the shaft of the CS can be limited by using a damp lint. Following complete preparation of the rectum up to the elevator ani, the stapler is positioned behind the bowel and by simple counter-clokwise motion the rectum is included between the CS jaws.

Result: The technique has been so far used in 21 cases of LRR. Fifteen patients had undergone previous neo-adjuvant treatment by chemo-radiation and in all these cases a 'ghost ileostomy' was prepared. Two anastomotic leaks occured (9.5\%).

Discussion: This result is very promising and we hope that a modificated design with an circular shaft can simplificate this techniques.

\section{9}

Laparoscopic Cholecystectomy in Day Surgery: Preliminary Experience of a First 80 Consecutive Cases

\author{
F.S. Mari, L. Masoni, M. Cosenza, A. Milillo, A. Brescia
}

UOD Day Surgery, Responsabile Prof. Antonio Brescia, UOC Chirurgia B, Responsabile Prof. Licinio Angelini,

Azienda Ospedaliera Sant'Andrea Universita' La Sapienza

di Roma - II Facolta' di Medicina e Chirurgia, Roma, Italy

Introduction: Laparoscopic Cholecystectomy (LC) represents today the gold standard for the treatment of cholelithiasis (CL). The reported average post-operative hospital stay is 2.6 days. Day Surgery (DS) LC has been therefore suggested.

Methods: Our treatment protocol for LC in DS includes age up to 65 years, $\mathrm{BMI}<35$, no major contraindications for the general anesthesia (ASA I or II) and no acute complications of CL. Between July 2004 and July 2007, 80 consecutive patients (pt) underwent DS LC. They were 27 males and 53 females, age ranging between 15 and 63 years. Patient motivations and home support was also investigated. They were admitted at 07:00. A 3-trocar technique was always used.

By 14:00 a soup is served and pt is allowed to walk. At 16:00 serum $\mathrm{RBC}$, total and direct bilirubin, amylase and lipase are checked. At 18:00 the patient is discharged with dietary instruction, drug prescription, and the telephone numbers of the operative unit.

Result: All the procedures were completed laparoscopically with a mean time of $38 \mathrm{~min}$. Only 3 (4.1\%) required overnight hospitalization for important nausea and vomiting. All patient but one expressed their satisfaction for this care regimen.

Discussion: The ambulatory LC is justificated by simplification of the procedure, reduction of management costs even over one-day surgery regimen and also by reduct impact upon patients' lifestyle.

\section{0}

\section{The Use of Staple Line Reiforcement in Laparoscopic Gastric Surgery: Our Experience with Seamguard}

\author{
F.S. Mari, L. Masoni, A. Brescia, M. Gasparrini, A. Milillo, \\ F. Favi, L. Angelini
}

UOC Chirurgia B, Responsabile Prof. Licinio Angelini, Azienda Ospedaliera Sant'Andrea Universita' La Sapienza di Roma - II Facolta' di Medicina e Chirurgia, Roma, Italy

Introduction: Bioabsorbable Seamguard (BSG) is a porous synthetic fibrous structure that is completely absorbed within 6 months or less. This prosthetic device has been used with good results in bariatric and thoracic surgery, representing a staple-line reinforcement to reduce staple-line hemorrage and to minimize the risk of leakage. The most frequent complication of the laparoscopic gastric surgery is staple-line leak and/or bleeding. Use of BSG may be useful in this last setting.

Methods: Between July 2004 and July 2007 we used the BSG in 21 laparoscopic gastric resection performed on 13 males and 8 females, age ranging from 49 to 86 years. The procedures performed were 11 gastric wedge resections, 6 partial distal gastrectomies, 4 total gastrectomies.

Results: None intraoperative or perioperative staple line complication were observed. No clinically relevant anastomotic leakage or bleeding were observed during the early postoperative period; in the first 16 patients no stenosis developed at 6 months follow-up. Mean hospital stay was 5.7 days (range 4-8).

Discussion: The use of BSG as a staple-line reinforcement seems safe and effective in preventing anastomotic leakage and bleeding. The bursting pressure of the anastomosis is increased. Low anastomotic complication rate allows hospital stay reduction and may justify the cost of the device. 
161

\section{Laparoscopic Wedge Resection of Parenchymal Organs: Our Experience}

F.S. Mari, L. Masoni, A. Brescia, A. Milillo, F. Favi, L. Angelini

UOC Chirurgia B, Responsabile Prof. Licinio Angelini, UOS Chirurgia dell'ano retto e pavimento pelvico, Responsabile Prof. Luigi Masoni, Azienda Ospedaliera Sant'Andrea Universita' La Sapienza di Roma - II Facolta' di Medicina e Chirurgia

Introduction: Laparoscopic wedge resection (LWR) of parenchymal organs (PO) can be performed safely in many instances. Bleeding and or postoperative bile leaks are the most common complications following these operations.

Methods: Between June 2006 and June 2007, 19 LWR of liver and of kidney were performed (11 males and 8 females). Mean age 55.7 yrs (range 31-68). They were 13 hepatic metastases and 6 renal cell carcinomas. Ultracision ${ }^{\circledR}$ and Ligasure ${ }^{\circledR}$ devices were used respectively in 15 and 4 cases. Hemostasis was always completed by using a combination of fibrin glue, gelatine matrix thrombin or fibrin sponge.

Results: All procedure were completed laparoscopically, mean operative time 115 minutes (range 75-190), mean blood loss $250 \mathrm{ml}$ (50-400), mean postoperative stay 5 days (3-8).

Discussion: LWR of PO using modern surgical instruments, ultrasonic or radiofrequency devices, with biologic hemostatic and sealant agent, is technically simple and safety and permits to decrease intra and postoperative complications. Indications are limited to resection of subglissonian lesions of II-III-IV-V-VI hepatic segments and of cortical renal nodules without involvement of pelvis.

\section{2}

\section{Laparoscopic Right Hemicolectomy with Caudo-Cranial Dissection. Original Techniques and Result of First $\mathbf{7 0}$ Consecutive Patients}

F.S. Mari, L. Masoni, A. Brescia, A. Milillo, F. Favi, L. Angelini

UOC Chirurgia B, Responsabile Prof. Licinio Angelini, UOS Chirurgia dell'ano retto e pavimento pelvico, Responsabile Prof. Luigi Masoni, Azienda Ospedaliera Sant'Andrea Universita' La Sapienza di Roma - II Facolta' di Medicina e Chirurgia

Introduction: Laparoscopic right hemicolectomy (LRH) is usually performed by a medial to lateral technique. Difficulties are represented by identification of the correct dissection plane. The $3 \mathrm{rd}$ part of the duodenum, an important landmark, in not easily identified in fat pts while in slim pts the mesentery of the right flexure is thin and fragile.

Methods: We have developed a technique of caudo-cranial dissection: (1) Identification and section of the ileo-colic vessels at their origin. (2) Division of mesentery up to the terminal ileum, which is cutted by EndoGIA stapler. (3) Incision of the Houston's legament. (4) Retroperitoneal dissection of the cecum and ascending colon up to the right flexure by pulling upwards the terminal ileum. (5) Incision of hepato-duodeno-colic legament. (6) Cranial traction of the specimen to identify and cut the right colic vessels and Henle's venous branch. In fat patients, section of the ileocolics vessels can be performed after preparation of the retroperitoneum up to the third part of the duodenum.

Results: This technique has been used in 70 pts: 38 men and 32 women. Mean age: 68.3 yrs and a mean BMI of 25.6 (22.3-37.4); the average time of operation was $125 \mathrm{~min}$, with no conversions. Mean postoperative hospital stay was 5.3 days. Two pts have an anastomotic leak.

Discussion: This technique is feasible, simple and reproducible.

\section{3}

\section{Laparoscopic Peritoneal Lavage in Perforated Sigmoid Diverticulitis}

\section{P. Mariani, L. Rampinelli, A. Guadalaxara, G. Quartierini, G. Perrone \\ Chirurgia Generale, Ospedale Bolognini, Brescia}

Introduction: The authors report their experience about the use of laparoscopic lavage in the management of generalized peritonitis in perforated sigmoid diverticulitis.

Patients and Methods: Fourteen patients, 8 males and 6 females with purulent peritonitis without fecal contamination (Hinchey II: 3 patients, Hinchey III: 11 patients), were treated with laparoscopic approach; perforated diverticular disease of the left coln was confirmed; laparoscopic extensive peritoneal lavage was performed and abdominal drains were inserted in all the patients.

Results: No intraoperative complications were noted in this cohort; all patients made a complete and fast recovery, with resumption of normal diet within 3 to 6 days. Main hospital stay was seven days.

Conclusion: Laparoscopic approach with extensive peritoneal lavage in the management of generalized peritonitis, in our experience, has to be considered as an important alternative to traditional open surgical approach.

\section{4}

\section{Ligasure versus Ultracision in Laparoscopic Colorectal Surgery: A Prospective Randomized Trial}

\author{
R. Rimonda, M.E. Allaix, G. Giraudo, M. Mistrangelo, \\ S. Birolo, M. Morino
}

General Surgery II, University of Turin, Dir Prof. M. Morino

Aims: This study was conducted to compare the efficacy and safety of LigaSure (LG, Valleylab) vs. Ultracision (UC, Ethicon Endo-Surgery Inc.) in laparoscopic colorectal surgery.

Methods: Patients eligible for elective laparoscopic right or left hemicolectomy ( $\mathrm{RH}$ and $\mathrm{LH}$ ) or low anterior resection of rectum (LAR) were randomized to either LG or UC. Endpoints were operative 
time, intraoperative blood loss and morbidity. A sample size of 140 patients was needed to have an appropriate sample size to prove a difference of $15 \%$ in operative time. Data collected and analyzed were: individual patient data, indication for surgery, conversion to open surgery, operative time, blood loss, complication rate, morbidity and mortality, volume of drainage, hospital stay.

Results: Between April 2005 and December 2006, 140 patients were included in this study. The diagnosis of these patients was diverticulitis (34), colonic neoplasm (103), endometriosis (1), rectal prolapse (1) and severe proctitis (1). Surgical procedures were: RH (16 UC and 15 LG), LH (32 UC and 37 LG), LAR (22 UC and 18 LG). There were no differences in preoperative data between groups. The overall conversion rate was $7.85 \%(11 / 140)$ (no differences between the two groups). No statistical differences were observed in terms of operative time (UC 117.5 vs. LG $124.2 \mathrm{~min}, \mathrm{p} 0.45$ ), blood loss (UC 106.8 vs. LG 109 cl, p 0.77), morbidity (UC 5.7 vs. LG 4.3\%, p 0.56), hospital stay.

Conclusion: LG and UC are useful instruments in performing the laparoscopic colic resection. No differences have been found between the two devices.

\section{5 \\ Morbidity and Mortality in Laparoscopic lleostomies and Colostomies, Retroprospective Study}

\author{
R. Rimonda, M. Mistrangelo, M.E. Allaix, D. Visconti, \\ M. Morino \\ General and Emergency Surgery II, University of Turin, Dir \\ Prof. M. Morino
}

Aims: The purpose of this study was to compare the morbidity and mortality observed in procedures of temporary and definitive stomas in colorectal laparoscopic surgery vs. open technique.

Methods: Retrospective study conducted on 40 consecutive patients treated with laparoscopic colonic resection and temporary or definitive ileostomy (26) and colostomy (14). We evaluated peri-operative (30 days) and post-operative complications.

Results: All patients presented a rectal neoplasia. Laparoscopic procedures were: anterior resection of rectum + TME $(70 \%)$, abdomino perineal amputation (APA, 25\%), Hartmann procedure (2.5\%), simple colostomy (2.5\%). The rate of peri-operative complications was $7.5 \%$, and they all required a second look. Among these 3 cases, $2(14.3 \%)$ were colostomies, only 1 was ileostomy $(3.84 \%$, $\mathrm{p}=0.59$ ). The mortality rate was $2.5 \%$ due to sepsis. During follow up the complicance rate was $40 \%$ : retraction $7.5 \%$, parastomal infection and partial detachment $10 \%$, prolapse $12.5 \%$ and stenosis $10 \%$. None of these complicances required another surgical procedure. Morbidity was observed in $38.4 \%$ of ileostomies, $42.8 \%$ of colostomies (no statistical differences). Recanalization rate was 51.7\%.

Conclusions: We can assume that the peri-operative and postoperative morbidity rate in laparoscopic ileostomies and colostomies is quite compared to open procedures.

\section{6}

\section{Laparoscopic Removal of Gastric Trichobezoar}

\author{
M.M. Napolitano, V. Bottino, I. Damiano, E. Scuderi, \\ E. Giuliano, P. Maida \\ Department of surgery 'Villa Betania Ospedale \\ Evangelico' - Naples, Italy, Head: P. Maida
}

Introduction: Gastric trichobezoar is a pathology secondary to trichophagy. It is often present in young women with psychiatric problems. The estimated incidence of bezoar is about $0.4 \%$; around 400 cases of trichobezoar have been reported in the literature. The treatment includes a conservative management with the use of a proteolytic enzymes and endoscopic disruption by using basket lithotripsy devices, and even laser. In some cases surgical intervention is the only way to remove a trichobezoar.

Methods: The study presents a case of a young woman with two great gastric trichobezoar treated with laparoscopic removal after the failure of an endoscopic treatment. Pneumoperitoneum was induced at the umbilical level with open technique and four trocars were used. Gastrotomy was performed with the hook coagulator in the anterior wall of the stomach. The two great trichobezoars were pulled out of the stomach and placed in two bags. The stomach was closed with Endogia stapler. The foreign bodies were extracted through the scar of the patient's previous appendicectomy.

Results: Post operative course was regular with discharge at eleven days. Gastric endoscopic follow up at one month showed a resolution of the case.

Discussion: Mini-invasive removal of gastric trichobezoar as the case reported by the authors is simple, safe, and effective. It represents a valid resolution in the case of a conservative management failure.

167

\section{Robotic Assisted Gastric Resection}

A. Patriti, G. Ceccarelli, A. Batoli, R. Bellochi, A. Spaziani, L. Casciola

\section{ASL 3, Ospedale San Matteo degli Infermi, Spoleto}

Introduction: Laparoscopic gastrectomy with lymph node dissection has been recently introduced as a treatment strategy for gastric cancer and oncological outcome measures comparable to open approach are reported. However, lymph node dissection remains the more demanding step of laparoscopic procedure. The present study was designed to verify if this limit could be overcame by a roboticassisted operation.

Material and Methods: 8 patients with histologically proved gastric cancer were enrolled. Six partial gastrectomy and 2 total gastrectomy were carried out during a span of time of one year. Tumor stage was as follow: Stage I in 5 and Stage II in 3 patients. Lymphectomy was carried out with the aid of the Da Vinci System.

Results: Mean operative time was 4.5 hours and a mean of $26 \pm 11$ lymph node were removed for each operation. Robot set up prolonged operative time of 30 minutes in average. No major morbidity or mortality was recorded. 
Conclusions: Robot assisted lymph node dissection is feasible and safe. Long term follow-up and randomized studies are needed to evaluate outcome of this new approach.

\section{8}

\section{Laparoscopy verus Computed Tomography (CT) in the Identification of Peritoneal Carcinomatosis (PC) from Colon Cancer and Others Abdominal Neoplasms: Retrospective Analysis}

\author{
E. Piazzese1, G.I. Mazzeo', S. Galipòn, F. Fiumara², \\ L.G. Angiò ${ }^{2}$ \\ ${ }^{1}$ Unità Funzionale di Chirurgia Laparoscopica, Clinica San \\ Camillo - Messina, ${ }^{2}$ Scuola di Specializzazione Chirurgia \\ Generale I, Università degli Studi di Messina
}

Background: The preoperative staging of the abdominal neoplasms uses some modern imaging techniques, that, despite the elevated sensibility, still introduces a meaningful limit in the diagnosis of the PC. The aim of this study was to evaluate the real effectiveness of laparoscopic procedure in the staging of abdominal neoplasms which were considered resectable at preoperative examinations and in particular in the detection of PC not evidenced with traditional imaging techniques as the Computed Tomography.

Methods: A series of 17 patients ( 10 female -7 male, mean age 61 years) submitted to laparoscopic surgery for suspected abdominal neoplasms ( 9 colon, 3 stomach, 2 ovary, 1 pancreas, 1 gallbladder, 1 liver) is retrospectively analyzed.

Results: All the patients were preoperatively examined with abdominal computed tomography (CT) and the suspicion of a PC has been formulated in the $17 \%$ of the cases (3/17 of patients). During laparoscopic procedure peritoneal washing and peritoneal biopsy was performed in all the patients. The diagnostic and therapeutic choices were subsequently done on the basis of intraoperative results. The PC has been documented through the explorative celioscopy in the $35 \%$ of the cases (6/17 of patients, 4 macroscopic and 2 microscopic); accordingly therapeutic approach was modified in $17 \%$ of cases, as a result of the detection of peritoneal micrometastases which were not evidenced with preoperative imaging examinations.

Conclusions: Laparoscopy performed in patients with abdominal neoplasms allows the detection of peritoneal micrometastases not previously evidenced through preoperative $\mathrm{CT}$, thus modifying the therapeutic approach (curative or palliative).
169

\section{Laparoscopic Fenestration (LF) for the Treatment of Patients with Symptomatic Simple Renal Cysts (SSRC)}

\author{
E. Piazzese1, G.I. Mazzeo', S. Galipò', F. Fiumara², \\ L.G. Angiò ${ }^{2}$ \\ ${ }^{1}$ Unità Funzionale di Chirurgia Laparoscopica, Clinica San \\ Camillo - Messina, ${ }^{2}$ Scuola di Specializzazione Chirurgia \\ Generale I, Università degli Studi di Messina
}

Introduction: Simple renal cyst is a common non-neoplastic disease of the renal parenchyma; $10-20 \%$ are symptomatic (flank pain, hypertension, haematuria, infection and obstruction of the upper urinary tract) and such cases are a major indication for intervention. LF of SSRC has been used since the early 1990s and the method is more effective than sclerotherapy. The purpose of this study was to present our experience in LF for patients with SSRC, analyze its feasibility and evaluate its immediate and mid-term outcome.

Methods: Between March 2005 and July 2007, 5 patients (mean age 54 years) with a SSRC were treated by LF. Patients were followed at 3-6-12 months after surgery and once a year thereafter; changes of cyst size after LF were determined by renal ultrasound and the serial changes were evaluated as the percentage of the diameter before surgery.

Results: Cyst size ranged from 6 to $14 \mathrm{~cm}$ (mean 7.5) and of these cysts, 4 were peripheral and 1 peripelvic. In all cases the LF was successful. The operative time ranged from 80 to 140 minutes (mean 105 ), and the mean blood loss was $<100 \mathrm{ml}$. The mean postoperative hospital stay was 3 days (range 2-7). There were no complications. At a mean follow-up of 11 months symptoms had resolved in all patients and radiological success rates were $80 \%$.

Conclusion: Although LF of SSRC is technically challenging, it is a safe and efficacious procedure and offers a favorable minimally invasive alternative for the treatment of symptomatic cysts.

170

\section{Incisional Hernia (IH) Laparosopically Repair with Parietex ${ }^{\circledR}$ Composite Mesh (PCM): Preliminary Result}

\author{
E. Piazzese1, G.I. Mazzeo1, S. Galipò', F. Fiumara², \\ L.G. Angiò ${ }^{2}$ \\ ${ }^{1}$ Unità Funzionale di Chirurgia Laparoscopica, Clinica San \\ Camillo - Messina, ${ }^{2}$ Scuola di Specializzazione Chirurgia \\ Generale I, Università degli Studi di Messina
}

Introduction: Repair of IH using the laparoscopic technique has continued to evolve since its inception. The biomaterials available have undergone many changes over the last several years; all seek to achieve two goals: rapid and permanent ingrowth into the prosthesis and diminution of the risk of intestinal adhesions. A new composite prosthesis denominated Parietex ${ }^{\circledR}$ Composite Mesh (PCM) was evaluated for IH laparoscopocally repair.

Methods: 21 patients (15 famale -6 male; range 38-67 years) underwent to laparoscopic repair for IH using PCM between March 2005 and June 2007. 
Results: The patients had undergone an average of 2.5 (range; 1-8) previous laparotomies. 3 repairs were performed for recurrent hernias and 4 for post-laparoscopy hernia. There were no conversions to open operation. The mean size of the defects was large at $90 \mathrm{~cm}^{2}$ (range 4-520). The mesh (average $302 \mathrm{~cm}^{2}$ ) was secured with metal tacks or resorbable staples. Operative time and estimated blood loss averaged $72 \mathrm{~min}$ (range 35-170) and $40 \mathrm{cc}$ (range 15-120). Length of stay averaged 2.5 days (range $1-4$ ). There were 4 minor complications: cellulitis in trocar site (2), seroma lasting $>4$ weeks (1), suture site pain $>2$ weeks (1). With an average follow-up of 14 months (range 2-26), no have been latter complication and recurrences.

Conclusion: From the results of our preliminary experience we can affirm that the laparoscopically employment of PCM is reliable and well tolerated for $\mathrm{IH}$; the further validation could derive from a longer follow-up and from comparison with some other prosthesic materials.

\section{1}

The Value of Laparoscopic Examinations (LE) in Diagnosis in Women with Chronic Pelvic Pain (CPP): Retrospective Study

\section{E. Piazzese1, G.I. Mazzeo', S. Galipòn', F. Fiumara², L.G. Angiò ${ }^{2}$ \\ ${ }^{1}$ Unità Funzionale di Chirurgia Laparoscopica, Clinica San Camillo - Messina, ${ }^{2}$ Scuola di Specializzazione Chirurgia \\ Generale I, Università degli Studi di Messina}

Introduction: CPP afflicts $5-10 \%$ of women. The cause's diagnosis is predominantly assessed by LE, which in $60-80 \%$ reveals various organic pathology; the source may be reproductive organ, urological, gastrointestinal, or myofascial.

Methods: This study analyses the results of $66 \mathrm{LE}$ indicated due to CPP. Patient's history characteristics, laparoscopic and cytologic findings were reviewed and analyzed.

Results: The average pain duration was 15 months (6-38) with the majority among women with history of previous surgery $(31.8 \%)$ and parturated women. Pain's presence was most common among women after 29 years of age. Abdominal organ pathology was present in $78.8 \%$ : endometriosis in 17 cases $(25.7 \%)$, adhesions without any other pathologic findings in 13 (19.7\%), chronic appendicitis in 8 $(12.1 \%)$, ovarial cysts in 5 (7.6\%), Chron's ileitis in 3 (4.5\%), hydrosalpinx in $2(3 \%)$, colon diverticulosis in $2(3 \%)$, varicose pelvic veins in $1(1.5 \%)$, uterine myoma in $1(1.5 \%)$. In 14 cases $(21.2 \%)$ no pathological change was revealed. LE was correlated with preoperative ultrasonographic findings in $36 / 52$ cases (69.2\%). Cytology examination of peritoneal fluid revealed an increased histiocytic reaction in coincidence with pain in $31.8 \%$.

Conclusion: Although LE is sometimes considered a routine part of the evaluation, ideally the decision to perform a laparoscopy should be based on the patient's history, physical examination and findings of non-invasive tests. Laparoscopy in coincidence with pain is a significant examination which helps to reveal the organic origin of disturbance. An serious decision of applying LE contributes to a correct diagnosis's assessment and treatment's commencement.
172

\section{Comparison of Suture Port and Velocity ${ }^{\circledR}$ Port: Retrospective Study on Perioperative and Short-Term Postoperative Implantation Outcomes}

\author{
V. Pilone, A. Formato, S. Tramontano, A. Monda, \\ M. Perone, P. Forestieri \\ Dipartimento di Chirurgia Generale, Università degli Studi \\ di Napoli Federico II, Naples, Italy
}

Introduction: Laparoscopic adjustable gastric banding is a safe and effective treatment method for morbid obesity. Port placement can take up to $10 \%$ of the overall laparoscopic adjustable gastric banding (LAGB) operating time, and inadequate technique can result in complications and prolonged pain. The purpose of this study was to evaluate the applicability and advantages of a port stapling device to simplify and improve port implantation compared to classic fixation with suture.

Methods: We retrospectively compared 30 cases of port implantation with Velocity ${ }^{\circledR}$ system with 30 cases of classic port implantation (standard fascial 3-point suture technique). No differences in mean age and body mass index were reported between the groups. For both groups we evaluated length of incision, time of implantation, rate of dislodgment, of infection, and pain (in postoperative phase and at 1 and 3 months), evaluated by recourse to drug and a four-graded pain scale.

Results: Incision length was longer in Velocity group $(\mathrm{p}<0.005)$. Port implantation time was significantly less using port stapling $(95 \pm 30 \mathrm{sec})$ compared to conventional port implantation $(510 \pm$ $140 \mathrm{sec}$ ). We observed two cases of dislodgment, 1 for group. Portsite infection occurred in a patient from the classic group and in none in the Velocity group. Reduction in pain complaints in favor of Velocity ${ }^{\circledR}$ port group was noted in postoperative period but no difference at 1 and 3 months p.o.

Discussion: Port implantation in LAGB is a very important part of the operation. Velocity ${ }^{\circledR}$ system led to reduction in time of implantation, pain on postoperative phase, but a longer incision length. Rate of dislodgment was acceptable for both techniques.

173

\section{Total Fundoplication is Effective in Controlling Extra-Esophageal Symptoms Resistant to Medical Therapy: Three Consecutive Patients with Combined MII-pH before and after Surgery}
S. Tolone, G. del Genio, G. Rossetti, L. Brusciano,
F. Russo, F. Pizza, C. Sagnelli, V. Maffettone,
V. Napolitano, F. Lucido, A. del Genio
I Division of General and Gastrointestinal Surgery, Second University of Naples, Naples, Italy

Introduction: Identifying and treating patients with supraesophageal symptoms is challenging. When the patient is not able to control his symptom with only pharmacological therapy, it could be 
indicated submitting to anti-reflux surgery. Laparoscopic fundoplication in fact is the gold standard in the surgical treatment of GERD, but still there are not studies which verify both the relieve of supra-esophageal symptom and the normalization of number and quality of reflux after surgery with the use of MII-pH. Aim of this study is to evaluate the outcomes of total fundoplication on relieving supra-esophageal symptoms and to verify the changes in $24 \mathrm{~h}$ MII-pH monitoring in pre- and post-operative period.

Methods: From January 2006 to May 2006, 12 patients complaining with extra-esophageal symptoms related to GERD were referred at our Institution. All of patients underwent to outpatient $24 \mathrm{~h}$ MII-pH before and after surgery. All patients selected for antireflux surgery underwent to the same procedure (laparoscopic NissenRossetti fundoplication LNRF). Data were collected prospectively at 3 and 6 months after LNRF.

Results: Of 12 patients, only 3 were eligible for the study. All of them underwent to LNRF and in all of them was performed clinical and instrumental follow up at 3 and 6 months. After LNRF the \% time esophageal $\mathrm{pH}<4$, both total, in upright or in recumbent position was very low. A statistical significance $(\mathrm{p}<0.05)$ was find in pre and post operative number of reflux detected at MII and in the number of refluxes detected at $15 \mathrm{~cm}$ MII segment.

Discussion: LNRF is a safe and effective procedure to protect against GERD and extra-esophageal symptoms. MII-pH is becoming the gold standard in diagnosing GERD and evidencing its relationship with extra-esophageal manifestations; after surgery MII-pH provides the right information to check the outcomes of surgery.

\section{4}

\section{Combined MII-pH is Useful in Selecting Patients for Laparoscopic Fundoplication}

\author{
S. Tolone, G. del Genio, G. Rossetti, L. Brusciano, \\ F. Russo, F. Pizza, C. Sagnelli, V. Maffettone, \\ V. Napolitano, F. Lucido, A. del Genio \\ I Division of General and Gastrointestinal Surgery, Second \\ University of Naples, Naples, Italy
}

Introduction: Laparoscopic fundoplication is the gold standard treatment in patients affected by Gastro-Esophageal Reflux Disease (GERD) not responsive to proton pump inhibitor (PPI) therapy at long-term. With the Combined Multichannel Intraluminal Impedance-pH (MII-pH), GERD can be better classified. MII-pH can identify acid ( $\mathrm{pH}<4)$, weakly acid $(4<\mathrm{pH}<7)$ or weakly alkaline $(\mathrm{pH}>7)$ reflux episodes. Also there is the possibility to have a Symptom Index with all type of refluxes. Aim of this study is to verify if indication to antireflux surgery provided by MII-pH results in a good clinical outcome.

Methods: From January 2006 to December 2006, 95 patients, complaining about symptoms of GERD, were referred to our Institution. All of them underwent to outpatient $24 \mathrm{~h}-\mathrm{MII}-\mathrm{pH}$. The positivity of $\%$ time $\mathrm{pH}<4$, total number of refluxes detected at MII $>73$, or a symptom association at least $50 \%$ were considered as parameters useful to indicate antireflux surgery. All patients selected for surgery underwent to the same procedure (laparoscopic Nissen-Rossetti fundoplication LNRF). Data dealing with symptom assessment were collected prospectively at 3 months after surgery.
Results: Of 95 patients investigated using MII-pH, 35.8\% $(34 \mathrm{pz})$ resulted positive for one or more parameters. In this group was indicated LNRF. Clinical follow up at 3 months from intervention was carried out in $97 \%$ of patients. $91 \%$ (31/33) were satisfied of the procedure. Mean postoperative modified DeMeester score was $0.2 \pm 0.2$ $(\mathrm{p}<0.05)$.

Discussion: The MII-pH is probably the new 'gold standard' in diagnosing GERD. Total fundoplication is the surgical way of treating GERD, controlling both acid and non-acid-refluxes. When there are right indications, the outcome of surgery is really high, up to $95 \%$.

\section{Maxillofacial Surgery}

\section{5}

Iliac Crest Flap: Donor Site Morbidity

I.V. Aboh, G. Longo, E. Brauner, A. Battisti, V. Valentini

Chirurgia Maxillo-Facciale Roma

Introduction: Starting from 80 's, thanks to the introduction of microsurgery, the reconstruction of wide and complex maxillomandibular osseous defects was treated with microvascular free flaps. Differently from the previous use of local flaps, grafts or alloplastics devices, free flaps allow to get a better functional and aesthetical restoration. Thanks to it's anatomical features, iliac crest, represent a flap of choice in head and neck reconstructive surgery.

Methods: The Authors present a retrospective analysis of their experience in the reconstruction of wide and complex maxillomandibular region defects. In particular they focus on the use of iliac crest flap. Harvesting surgical technique is discussed. An international review of donor site morbidity is carried out, and it's compared with authors' own experience.

Results: A variety of complications associated with iliac crest flap harvesting have been reported in literature, including: chronic pain; sensory loss; paresthesia; haematoma; seroma; gait disturbance; instability of the sacroiliac joints; pathologic fracture; contour defect; regional scars.

Discussion: Composite iliac crest tissue transfer has an established role in head and neck reconstructive surgery. While maxillomandibular reconstruction outcomes, in terms of functionality and aesthetic restoration have been variably reported, few studies have treated donor site complications and morbidity. The present work deals with such aspects. 


\section{6}

An Orthodontic-Orthognathic Conceptual Approach to OSAS Surgery: Our Experience and Results

\author{
F. Laganà, D. Ali Youssef, A. Baj, A.B. Gianni \\ Maxillofacial surgery, Department of Clinical Science \\ 'Luigi Sacco' University of Milano, I.R.C.C.S Istituto \\ Ortopedico Galeazzi
}

Introduction: Obstructive sleep apnea syndrome (O.S.A.S.) ride on the upper airway obstruction during sleep. The techniques, borrowed from orthognathic surgery, have great success in OSAS surgery. Maxillo-mandibular advancement (MMA) is successfull in $90-100 \%$ of cases

Methods: Pre surgical orthodontic treatment followed by orthognathic surgery improves the aesthetic and functional results. The authors present a 10 patients serie; presurgical RDI average was 56 and all patients report the majorty of clinical signs and symptoms. Different OSAS related maxillo-mandibular conditions composed the serie.

Results: After surgery the RDI average was 6 and the clinical recovery were assessed for all the serie. MMA concern with the forward reposition of the MM complex preserving the personal dental occlusion. However, the majorty of patients with mandibular ipoplasia have a dental uncorrect occlusion, too. A continuous positive airway pressure (CPAP) support, during presurgical orthodontic preparation, allow us to spend time for normalize the dental arches to obtain the best skeletal position and dental occlusion at surgery.

Discussion: Presurgical orthodontic treatment advantage eumorphic patients too. It entail a more stable dental occlusion that contrast the deterioration tendency of the results. Pre-surgical orthodontic treatment qualify orthognathic surgery even in OSAS patients.

\section{7}

\section{Osteonecrosis of the Jaws Bisphosphonate- Induced: What's Therapy for a Better Quality of Life?}

G. Ambrosio, T. Bucci, G. Dell'Aversana Orabona,

A. Romano, F. De Giulio, E. Pavone, S. De Biasi,

A. Santella, L. Califano

Federico II University of Naples, Italy, Department of

Maxillofacial Surgery, Director: Prof. Luigi Califano

Introduction: Bisphosphonates, pyrophosphate analogs, are strong osteoclast inhibitors that are widely used in the management of metastatic disease to the bone and in the treatment of osteoporosis. In the last years a number of cases of osteonecrosis of the jaws (ONJ) associated with these drugs have been reported.

Methods: We present a series of 18 patients with bisphosphonaterelated ONJ. The typical presenting symptoms were pain and exposed bone at the site of a previous tooth extraction. Ten bone exposures occurred in the mandible alone, 5 in the maxilla, and 3 occurred in both jaws.

Results: Patients who showed regions of exposed and necrotic bone but were asymptomatic have been followed and treated conservatively with clorhexidine mouthwashes. However, 7 patients required surgical procedures to remove all of the involved bone that was a constant source of infection and was not responsive to irrigations and antibiotic therapy. The procedures included 2 sequestrectomies, 1 marginal mandibular resections, 3 segmental mandibular resections and 1 complete maxillectomy.

Discussion: The management of these patients with ONJ remains extremely difficult. We support the fact of the need to establish preventive measures in all the patients projected for intravenous bisphosphonate treatment. These patients should undergo a specialist oro-maxillofacial or dental check up before treatment beginning in order to prevent ONJ. According to international literature we believe that surgical treatment should be recommended in those refractory cases to non-surgical treatment and if sypmtoms continue.

\section{8 \\ Reconstructive Options in Lateral Mandibulectomy}

\author{
G. Beltramini, A. Baj, A.B. Gianni \\ Chirurgia Maxillo-faciale, Campania
}

Post-oncological reconstruction of lateral mandibular defects still presents a challenge for the surgeon. This work focalized the attention on those cases in which the patient could not undergo reconstruction with free flap containing bone. We present 5 cases of post-oncological reconstruction with locoregional flap with or without reconstructive plates. We porformed 35 partial or total mandibulectomy since 2002. Only in five cases, beacuse of their performance status, we didn't choose for microsurgical reconstruction. In 3 patients with N0 we have reconstructed the defect with submental flap with or without plate, while in two cases with $\mathrm{N}+$ we performed a pectoralis major flap only in case with plate. Follow up lasted from a minimum of $1.5 \mathrm{yrs}$ to $3.5 \mathrm{yrs}$. All patients are free from desease, preserve a good masticatory function and are satisfied of the aesthetic result. The reconstructive's aim in lateral mandibulectomy is to separate the neck from oral cavity. In those patients that couldn't undergo microsurgical reconstruction the surgeon could choose between locoregional flap such as submental's one if the neck is free from desease. Otherway also pectoralis major gives a good quality of tissue for oral reconstruction.

\section{9}

\section{Lip and Ear Amputation: Microsurgical Replantation and Primary Reconstruction with Local Flap. Our Experience}

\author{
P. Formillo, A. Baj, F. Laganà, A.B. Giannì \\ Istituto Ortopedico Galeazzi, Milano
}

Introduction: Facial tissue amputation is a rare and drammatic event that involve both aesthetic and functional aspects. When is possible microsurgical replantation must be attempt in order to restore in 
one-stage surgery both form and function. Other reconstructive options are either available using locoregional flap.

Methods: We treated 4 patients with facial amputation: 3 patients with lip amputation, 1 patient with partial ear amputation. In only one case microsurgical replantation of the lip was succesfully performed while the other defects was reconstructed with local flap such as Famm flap and Mladick thecnique. In three cases a second stage surgery was performed after one month to 'take down' the pedicle.

Results: In all four cases we obtained a satisfactory healing af the flap, with no infection, dehiscence or partial necrosis. One patient required a secondary treatment one year after lip replantation in order to restore vermilion's defect secondary to trauma. In all patients both aesthetic and functional aspects were restore.

Discussion: Early repair of avulsive facial wounds is mandatory. In this kind of trauma microsurgical replantation is the gold standard even if the post operative care is harder. When this technique is not available local flaps provide a reliable reconstructive option for early repair of soft tissue defect secondary to trauma.

\section{0 \\ Restoration of Nasal Defects: Analysis of $\mathbf{3 3}$ Cases}

E. lacomino, E. Facciuto, G.M. Mangone, M. Cassese

Unit of Maxillo-Facial Surgery, 'A. Cardarelli Hospital' Naples (Head: Dr. S. Parascandolo)

Introduction: The position of the nose as the focal point of the face makes its reconstruction a procedure requiring acute attention to detail and to preservation of the nasal 3-dimensional integrity.

Material and Methods: Thirty-three patients who had undergone nasal reconstruction following excision of basal cell (27), squamous cell (3) carcinomas and complex trauma (3) were reviewed. The mean age of oncologic group of patients was 67 years, of traumatic group was 28 . The most common site of involvement was the nasal tip (40\%), followed by the nasal ala (25\%), nasal dorsum (20\%), and the nasal sidewall $(5 \%)$. In each case, reconstructive options were considered after careful evaluation of the tissue quality and laxity adjacent to the nasal defect.

Results: The most common procedure performed was 13 primary closures (40\%), 10 skin grafts $(30 \%), 9$ local flap procedures (27\%), included 3 midline forehead flaps, 2 Rieger-Marchac flaps, 2 nasolabial flaps, 1 nasal-cheek flap, 1 island forehead flap. Just in one case $(3 \%)$ was used a radial free flap. At a mean follow-up of 10 months, all flaps healed uneventfully.

Conclusions: Other flaps may be used to reconstruct these nasal defects with comparable results. However, in our experience, the flaps presented here have reliably produced excellent aesthetic and functional results.
181

\section{The Two Mini-Plate-Fixation Technique in Condylar Neck Fractures}

\author{
A. Spinzia, L. Liccardo, V. Pagliarulo, S. De Biasi, U. Tozzi \\ P. Piombino \\ Università degli Studi di Napoli Federico II, Cattedra e \\ Scuola di Specializzazione in Chirurgia Maxillo-Facciale, \\ Direttore: Prof. Luigi Califano
}

Introduction: The most common fractures in the maxillo-facial region are localized in the mandibular condylar process $(1,2)$. The main method is closed reduction with functional therapy, although open reduction and internal fixation are required for treatment in case of severe displacement or dislocation of the condyle (3).

Methods: In literature, a lot of fixation techniques have been described, such as intraosseus wiring, Kirschner wires, miniplates and lag screws $(4,5,6,7)$. According with Champy's technique, in our study we used the two-miniplate-fixation-technique, applied at the posterior border and antero-lateral border of the condylar neck, in 18 patients with a mean follow-up of six to twelve months. We evaluated data about facial nerve functionality, joint mobility, dental occlusion.

Results: In our patients the facial nerve functionality and dental occlusion were preserved, joint mobility was physiologic but deficient in the contralateral deviation.

Discussion: Strain measurements in the condylar process showed that the highest level of tensile strain was supported by the anterior and lateral surfaces and the highest compressive strain by the posterior surface (8). The condylar neck fractures treated with twominiplates showed to have the beneficial effect of restoring the tension and the compression trajectories. Although two-miniplate fixation might be more traumatic than other techniques, we conclude that this technique is the better to achieve early mobility of the jaw and stability of the fracture site, when open reduction is indicated in cases of condylar fractures.

182

\section{Castleman's Disease}

L. Liccardo, A. Spinzia, G.M. Mangone, S. De Biasi,
V. Pagliarulo, L. Lanero, P. Piombino

Università degli Studi di Napoli Federico II, Cattedra e Scuola di Specializzazione in Chirurgia Maxillo-Facciale, Direttore: Prof. Luigi Califano

Introduction: Castleman's disease is an uncommon disease of lymphoid tissue that typically presents as a solitary mediastinal mass. It was first described by Castleman et al. in 1956. The head and neck regions are the second most common areas for giant lymph node hyperplasia after the mediastinum.

Methods: A 35-year-old woman with a right parotid mass was first seen in February 2006. Physical examination of the parotid region showed that the mass, its dimensions were $4 \times 3 \mathrm{~cm}$, it was mobile and nonpulsatile, with a rubbery firm consistency and well defined borders. Computed tomography of the neck confirmed the presence of the mass. Fine-needle aspiration biopsy revealed a mixed 
population of lymphoid cells: a specific reactive hyperplasia. The patient underwent excision of the neck mass in April 2006.

Results: We report one case of Castleman's disease in the parotid region. This pathology usually presents as an asymptomatic and solitary mass. Preoperative workup includes radiographic studies and fine needle aspiration biopsy. Final diagnosis was made by histopathological evaluation of the excised specimen.

Discussion: Castleman's disease is typically affects young adults between 15 and 35 years of age with no sex predilection. The pathogenesis of Castleman's disease is poorly understood. A definite diagnosis of the disease is made on histological analysis. Treatment is by excisional biopsy and appears to be curative; in inoperable or systemic variants the treatment is radiation therapy and chemotherapy.

\section{3}

\section{Preoperative Assessment of Salivary Gland Tumours with Ultrasonography with Second Generation Contrast Medium}

\section{F. Longo', C. Aversa', L. Leparulo², F. Sandomenico ${ }^{3}$, F. lonna ${ }^{1}$ \\ ${ }^{1}$ S.C. Chirurgia Maxillo-facciale e ORL Istituto Nazionale dei Tumori di Napoli - Fondazione}

Introduction: Ultrasonography with second generation contrast medium (Sonovue ${ }^{\circledR}$ ) offers new potentiality in the preoperative study of salivary gland tumour.

Methods: 25 consecutive patients (age $25-75$ years) affected by salivary gland tumours are evaluated. The patients underwent ultrasonography with Sonovue before surgical intervention. Histology after surgery was Warthin tumour in 8 cases, pleomorfhic adenoma in 10 cases, and neurinoma, lipoma, liposarcoma, cilindroma, adenocarcinoma, indifferentiated carcinoma in one case each.

Results: In case of benign tumour diffuse nodular impregnation with slow dropping of contrast medium was observed; in malignancies intense and inhomogeneous impregnation with rapid contrast dropping was considered typical. Preoperative diagnostic accuracy of ecocontrastography was of $88 \%$ (22/25 cases); the unfavourable cases were one case of adenoid cystic carcinoma for the presence of diffuse cystic componenent; one case of neurinoma confused with a pleomorphic adenoma for the presence of a vascular pattern 'basket like'; and one case of well differentiated liposarcoma for the presence of lipomatous tissue.

Discussion: Ultrasonography with second generation contrast medium can add new and important elements in differential diagnosis for the preoperative assessment of salivary gland tumours.

\section{4}

\section{Bilateral Facial Paralysis Reanimation with Gracilis Free Flap in A Case of Moebius's Syndrome}

\author{
A. Longo, I.V. Aboh, E. Brauner, A. Battisti, P. Gennaro, \\ V. Valentini \\ Chirurgia Maxillo-Faciale, Policlinico Umberto I, Roma
}

Introduction: The Moebius's Syndrome is a rare congenital pathology characterized by oculo-facial paralysis whose clinical picture is dominated from the facial amimia, inability to smile, to blink and to move the eyes sideways. Such syndrome is due to an anomaly of development of the VII c.n, that it is also associated to anomaly of the VI c.n. in $75 \%$ of the cases.

Methods: The authors introduce the clinical case of a young patient suffering from Moebius's Syndrome, who underwent a bilateral reanimation of the VII c.n. with microvascular gracilis free flap in the Department of Maxillo-Facial surgery of 'La Sapienza' University of Rome. They performed two operations with an interval of time of nine months in order to restore the facial expressions and especially the ability to smile.

Results: Follow up included fisiotherapy. After a period of 7-8 months we recorded a satisfactory functional results and the patient became able to smile.

Discussion: Microneurovascular free muscle transfer is fast becoming a standardized procedure in the treatment of established or long-standing facial paralysis. However, there is no general agreement about which is the best muscular free flap to use. In this clinical case we have chosen microvascular gracilis free flap. The reasons of this choice were: thinness of the flap, reduction of operative time and possibility of working in double equipe, good aesthetical and functional outcomes of donor site.

\section{5 \\ Retromandibular Surgical Approach in the Condilar Fractures}

M. Friscia, T. Bucci, G. Ambrosio, A. Santella, S. De Biase, A. Mentone, P. Graziano, M. Maietta, L. Califano

Department of Oral and Maxillofacial Surgery, University of Naples 'Federico II', Italy

Introduction: Post-traumatic fractures of the condilar region represent a third of all the mandibular fractures, they are able be of three types, happen, cervical and basi-cervical.

Methods: The treatment reserved to the condilar fractures are a lot of controversial, in fact it is tendency effect the functional conservative treatment in the capitate fractures, while the surgical treatment is reserved for the cervical fractures and basicervical.

Results: From March 2002 to March 2003 in our department we treated 14 patients, 13 with monolateral sottocondilar fractures with displacement of the condilar bone and 1 with bilateral fracture. All were treated with rigid fixation and with retromandibular surgical approach. We effected follow-up to 3, 6 monthes and to 1 years, we appraised some parameters like the stoppage, the articolar function, condilar 
changes, nervous lesions and finally the cutaneous scar. In the checkups to distance the patients introduced a good opening of the mouth, good movements of laterality, good resulted be even the stoppage. Besides the functionality of the facial is been altered in any of the 14 patients. essays, finally the cutaneous scar was nearly imperceptible.

Discussion: The end point of this work is to appraise the retromandibular acces, in the extracapsular condilar fractures with removal of the distal fragment. We want to show the facility and the control of this technique, because of the facility of execution and the safety on possible lesions in charge of the facial nerve.

\section{6}

Sinus Lift: Filling Materials in Comparison

G. Nicolai, B. Lorè, G. Mariani, L. Calabrese

Policlinico Tor Vergata, Roma

Introduction: In 1980 Boyne and James first described this method. They used spongious bone grafts taken from the iliac crest and transformed a part of the pneumatic cavity of the sinus into osseous neo-tissue which would have allowed the insertion of fixtures and an implant-prosthetic ideal rehabilitation. Currently we have at our disposal a wide range of filling materials which differ on their origin and on their characteristics. In our opinion the iliac crest represents the best solution because of its remarkable osteoconductive and osteoinductive properties. A sound alternative is the deantigenated bovine bone.

Methods: The Authors of this article describe some emblematic cases of Sinus Lift comparing the two most popular filling materials: The autologous bone taken from iliac crest and the heterologous bone of bovine origin.

Results: In all the cases treated we obtained good results in terms either of mineralization and of implant-prosthetic rehabilitation.

Discussion: Nowadays the sinus lift still represents the most effective solution to obtain a good implant-prosthetic rehabilitation in the edentulisms of the postero-lateral sectors of the maxilla. Despite the autologous bone taken from the iliac crest represents the reference material, it presents a double disadvantage: A certain degree of morbidity to the donor site and an higher cost (it is necessary to perform the taking of it in an operating room) if compared to non-autologous materials, in particular the heterologous bone of bovine origin which, despite being only osteoconductive, represents a sound alternative for the above mentioned reasons.

\section{7}

\section{Open Bite Surgical Adjustment in Vertical Development Excesses of the Maxilla}

\author{
G. Nicolai, B. Lorè, E. Ventucci, L. Calabrese \\ Policlinico Tor Vergata, Roma
}

Introduction: Maxilla may be totally or partially overdeveloped in the vertical dimension. The entire maxilla may be located in a lower position compared to the cranial base or its excessive development may totally or partially concern its posterior or anterior part. In the event of an overdevelopment of the posterior part, there is a clockwise rotation of the lower jaw which favours the formation of an anterior open bite: In such case the occlusal contact will occur only in the back, resulting in an increase in the front facial height (long face). The anterior vertical maxilla overdevelopment instead, causes a moderate clockwise rotation of the lower jaw and a posterior open bite.

Methods: In this article the Authors present their experience in the surgical treatment of the open bite in the maxilla vertical development excesses.

Results: In all the cases treated we obtained an open bite correction with satisfying aesthetics and functional results for the majority of the patients.

Discussion: Our school surgical correction of the open bite in the vertical over-development of the maxilla involves a parallel or a wedge osteotomy with posterior or anterior base according to whether an elevation of the whole maxilla or an elevation respectively of the anterior or posterior part is to be achieved. The measures of the movements are determined in the pre-surgical phase with a cephalometry, the VTO and model set-up. Where necessary, a BSSO of the lower jaw is performed for restoring the correct dental occlusion.

\section{8}

\section{Post Surgical Operation Management in Reconstructive Surgery of the Cervico-Facial Region with Microvascular Free Flaps: New Frontiers}

\author{
G. Nicolai, B. Lorè, A. Saltarel, L. Calabrese \\ Policlinico Tor Vergata, Roma
}

Introduction: In this work the Authors, on the basis of their experience in the field of reconstructive microvascular surgery, wish to show the guidelines adopted in the post surgery management of patients who underwent reconstructive surgery of head and neck deficit with microvascular free flaps.

Methods: Post surgery management aims at ensuring the viability of the tissues of the free flap. It basically depends on the efficiency of the vascular pedicle and on the consequent functioning of the microcirculation. It is therefore important both to prevent the compression of the pedicle and to preserve a good 'blood fluidity'. In our opinion the best pharmacological aids for this goal: a plasma expander to be used in case an excessive concentration of the blood cellular component arises; an antiaggregant. The most used wide spectrum intravenous antibiotic therapy was the association of amoxicillin and clavulanic acid with metronidazole, as in our opinion it was the most reliable. An alternative scheme implies the use of clindamycin and ciprofloxacin.

Results: In the last four years, in the maxillo-facial surgery department of 'La Sapienza' and 'Tor Vergata' Universities, 122 facial bone deficit reconstructions with free flaps were performed, with success percentages exceeding $95 \%$.

Discussion: In this study the Authors propose a clinicalinstrumental and pharmacological protocol widely experimented in our School which contributed to the obtainment of success percentages in line with the results of international literature in the field of reconstructive microsurgery. 


\section{9}

Treatment of Cranio-Maxillo-Facial Trauma: Which Kind of Intubation?

\author{
V. Palazzolo, S. Spotti, S. Valsecchi, C. Gervasoni, P. Ronchi \\ Chirurgia Maxillo-Facciale, Milano
}

Introduction: The orotracheal intubation, in the treatment of severe cranio-maxillo-facial fractures, doesn't allow a correct occlusion's restoration and make it hard the reposition of the maxilla and the mandible; the naso-tracheal intubation is impossible when the patient has nasal or skull base fractures. Cricothyroidotomy or tracheotomy, recommended when oral or nasotracheal intubation are not possible, are invasive procedures with high morbidity, but make easier the surgery in this kind of operations. In the treatment of trauma, when intermaxillary rigid fixation is necessary, a valid alternative is rappresented by submental intubation, described first by Altemir in 1986 .

Methods: From January 2002 to December 2006, Altemir intubation was performed in 27 patients affected by mid-facial fractures. Execution's duration, intraoperatory complications and follow-up results were valued.

Results: In our experience, submental intubation didn't show intra or post surgical complications. Scars, nervous or gland lesion and compromission of oral floor were not finded in all the patients.

Discussion: The submental intubation is recommanded in the treatment of mid-face trauma that doesn't required post-surgical ventilatory assistance and that required short period of intermaxillary rigid fixation. The surgeon, anaesthesist and nurse collaboration allows an easy, fast and safe procedure.

\section{0 \\ Management of Palatal Perforation Induced by Cocaine Snorting}

\section{R. Rauso, L. Corzo}

Department of Pathology, Head, Neck and Oral Surgery, Service of Maxillo Facial Surgery, Service of Maxillo Facial Traumatology, Second University of Naples, Naples, Italy

Introduction: Cocaine is a naturally occuring alkaloid. It is extracted from the leaves of the Erythroxylon coca plant. Cocaine is a psychologically distruptive and dependence-inducing drug; classified as a psychostimulant, it exhibits both local anesthetic and neurotrasmitter effects. Cocaine usage has a growing incidence worldwide. The most common route of administration is nasal that induces local vasoconstiction and irritation on the exposed tissue due to the effects of both the active substance and others added during its manufacture. Repeated snorting sets up a cascade of ischemia, inflammation, micronecrosis, infection and then macronecrosis leading to perforation. Authors describes their experience in diagnostic management of cocaine addicted with oro-maxillo-facial lesions and present the surgical treatment of a case.

Methods: Three cases of palatal perforation induced by cocaine snorting are presented.
Results: Only a case was admitted for surgical reconstruction. One other case was treated with a palatal obturator and the other one missed to return during the diagnostic management.

Discussion: Management in cocaine cases is not an easy task due to the non-compliant lifestyle of the typical patient. Authors affirm that surgical management for this patients could be done with a lot of techniques such as free flap, but because of this patients are generally in a bad health status and they are noncompliant, surgical treatment must be the less invasive is possible or management has to be done with non surgical treatment such as palatine obturator.

\section{1}

\section{Diagnostic Validity and Therapeutic Effect in Patients Submit to 'Sialoendoscopic Treatment' for Obstructive Pathologies of the Majors Salivary Glands}

\author{
R. Rausos ${ }^{1}$, A. Vicidomini', G. Colella', P. Bove', \\ G. Campisi ${ }^{1}$, C. Di Liberto ${ }^{2}$, R. Speciale ${ }^{2}$ \\ 1Dipartimento Testa e Collo, Cavo Orale e Maxillo- \\ Facciale, Seconda Università degli Studi di Napoli; \\ 2Dipartimento di Scienze Odontostomatologiche ed ORL, \\ Università di Palermo
}

Introduction: The obstructive 'scialoadeniti' constitute the prevailing infiammatory pathology of the major salivary glands. The demonstration that is possible a residuals grapes's regeneration and news grapes's making after the experimental atrophy induced by ductal ligatures constitute the theoretic base for the mininvasive tecnique's development. The target of this study is to show the diagnostic validity and the therapeutic effect in patients underwent a sialoendoscopic treatment for obstructive pathologies.

Methods: At the time between March 2005-July 2007 in the Naples's University -SUN- (Max-Facc Surgery) and in Palermo's University (MO and ORL) we submit to sialoendoscopic diagnostic and therapeutic treatment 100 patients with glands obstructive patology not in acute fase. In the pre-operative value all the patients was submit to echographic and radiographic exame.

Results: In almost all the patients that was submit to sialoendoscopy $(90 \%)$ we make sure the cause of the obstruction and we formulate related demission diagnosis. The diagnostic SE was implement with positive results in $90 / 100(90 \%)$ and negative results (impracticability exam) in 10/100 (10\%). The operative SE was implement in 60 patients with positive results in 50 patients; 45 of this with improvement of the syndrome and 5 of this with complete symptomatology's remission. 8 patients are shown recidive in about 1-3months and was submit to a second SE with positive results.

Discussion: The results of this study show the effectiveness of the methodology in the diagnosis and terapy of the major salivary gland's disease correlates to ductal obstructions, with a minimum exam's invasivity and with the possibility to act in systemic pathology. The accurate selection oh the case examinee to the SE is a fundamental condition for the positive results of the treatment. 


\section{2}

\section{Post-Surgical Morfo-Functional Evaluation in Patients with II Class Dentoscheletrical Deformity}

\author{
D. Rocchetta1, F. Laganà1, C. Sforza², F. Ferrario², \\ A.B. Gianni \\ Istituto Ortopedico Galeazzi, Milano
}

The surgical and orthodontic treatment of patients with morphological alterations of craniofacial district has two objectives: improve aesthetic morphology and obtain functional results. Today the technology allows fine measurements of facial morphology and functions of temporomandibular joint compartment. The study has considered a group of 20 patients affected by skeletal Class II patients treated surgically (mandibular increasing by sagittal split osteotomy and LeFort I maxillary osteotomy). The patients were analyzed morphologically and functional at 9 months after surgery. The functional aspect was evaluated with an automated infrared analysis of tmj compartment which permits the evaluation of the translational and gliding movements. The facial volumetric changes were assessed using a non-invasive three-dimensional digitizer of 50 facial landmarks. Surgery increased mandibular volume (approximately, a significant, average 15\% increment), but it reduced maxillary volume (an average 13\% reduction). The tmj function has considered stable and with no difference compared with the same value before surgery. This method permits not only the evaluation of patients but also the measurements of normal people to create functional and morphological values to be used as referential in others studies. The present research showed that the 3-D quantitative assessment of facial soft tissues could possess some potentialities as like as the evaluation of the tmj movements in maxillofacial and orthognathic surgery.

193

Extremely Atrophy of the Maxilla: Results and Indications of Modified Sailer's Technique

\author{
S. Spotti, V. Palazzolo, C. Gervasoni, A. Di Francesco, \\ P. Ronchi \\ Az. Osp. Sant'Anna, Az. Osp. Sant'Anna, Milano
}

Aim: The purpose of this clinical investigation and follow-up was to report the clinical outcome and consequently indications of osseointegrated implants placed in extremely atrophied edentulous maxillae after Le Fort I osteotomy and interpositional autogenous iliac bone grafts.

Patients and Methods: A two-step procedure made by Le Fort I osteotomy, downtilting of the maxilla, interposition of chips and splint of iliac bone graft in the sinus were performed in 25 patient and placed. 6 to 8 months after the reconstructive procedure, 175 osseointegrated implants were placed in the reconstructed maxillae. 6 to 8 months afterwards, abutments were connected and the prosthetic rehabilitation started.

Results: The reconstructive procedure was successful in 24 of 25 patients. In one patient, almost total loss of the inlay graft occurred before implant placement, due to the inappropriate and extended use of partial denture. 6 patients dropped out of the study ( 5 die of other causes). 10 implants were removed during the follow-up period, due to loss of integration. 20 implants, although placed, presented with peri-implant bone-level changes higher than those proposed for successful implants. Cumulative survival and success rates of implants were $94.3 \%$ and $82.9 \%$, respectively.

Conclusion: Results from this study showed that Le Fort I osteotomy with interpositional bone grafts followed by delayed implant placement is an acceptable means to rehabilitate edentulous patients affected by extremely atrophied edentulous maxillae only for specific conditions: under 60 years old patients, $2-3$ month partial dental proscription.

\section{4 \\ Diagnosis and Treatment of Parapharyngeal Space Benign Tumors

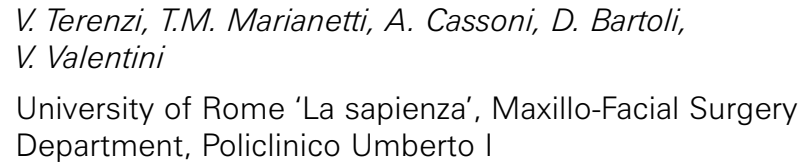

Introduction: Approximately $80 \%$ of neoplasms arising in the parapharyngeal space are benign lesions. Various surgical approaches, eventually associated with mandibulotomy to increase exposure, have been described.

Methods: From 1996 to 2007, 50 pts presenting a PPS mass underwent surgery at our centre. Pre-operative imaging consisted of CT scan and/or MRI study; in 16 cases fine-needle aspiration was performed. Follow-up ranged from 6 months to $10 \mathrm{yrs}$.

Results: In 32 cases (64\%) masses originated from the deep lobe of the parotid gland. Six pts with recurrent pleomorphic adenoma had undergone previous surgery involving the same anatomic region. In only one case PPS mass (pleomorphic adenoma of the deep parotid gland) was diagnosticated incidentally during tyroid ecography exam, while in other cases initial manifestation was a mass in the upper neck. Between the pts which underwent pre-operative FNA, an accurate preoperative diagnosis was done in $54 \%$ of cases. Various surgical approaches were used, eventually associated with mandibulotomy:

(a) transcervical approach $(\mathrm{n}=16)$ : in 1 case with mandibulotomy

(b) transparotid approach $(\mathrm{n}=30)$

(c) transcervical-transparotid approach $(n=2)$ : in 1 case with mandibulotomy

(d) transcervical-transoral approach $(\mathrm{n}=2)$ : in 1 case with mandibulotomy.

Discussion: Transcervical approach led to an optimal mass exposure, while transparotid approach is the gold standard when facial nerve dissection is required. Pre-operative fine-needle aspiration have not demonstrated to be an accurate method for diagnosis. 
Neurosurgery

\section{5}

\section{Splitting of the Optic Nerve-Optic Chiasm Complex caused by a Pituitary Macroadenoma}

\author{
A. D'Andrea, R. Zappia, P. Merciadri, M. Bruzzo, G. Zona
}

U.O. Clinica Neurochirurgica e Neurotraumatologica, Azienda Ospedaliera Universitaria 'San Martino', Genova (Dir. Prof. Renato Spaziante)

Introduction: Fenestration of the optic chiasm or the optic nerve by intrachiasmatic craniopharyngiomas or anterior communicating aneurysms is a rare condition already reported in the literature. To our knowledge, this is the firs report of such a condition caused by a pituitary macradaenoma.

Material: A young lady presenting with a non-functioning pituitary macroadenoma with suprasellar extension and no visual field deficits was first operated on be the transsphenoidal route with emptying of the sellar portion of the tumor. Six months later, as non spontaneous descent of the suprasellar part was observed, the patient underwent a second operation by a transcranial pterional approach. During the procedure a spontaneous inverted Y-shaped splitting of the right optic nerve and chiasm was observed, completely embedding the tumor, with no clear cleavage plane. Such a condition was believed to be responsible for the missed spontaneous intra sellar descent of the lesion.

Discussion: Neuroradiological features as well as possible etiopathogenetic hypotheses of this rare condition are discussed.

\section{6}

\section{Endoscopic Kysto-Maxillo-Sphenoidostomy as Salvage Therapy in a Recurrent Cystic Craniopharyngioma. Case Report}

\author{
A. D'Andrea', E. Castel/o², D.C. Rossi', A. Ali ${ }^{1}$, \\ R. Spaziante \\ ${ }^{1}$ U.O. Clinica Neurochirurgica e Neurotraumatologica, \\ Azienda Ospedaliera Universitaria 'San Martino', Genova \\ (Dir. Prof. Renato Spaziante); '2U.O.S. Otorinolaringoiatria, \\ Azienda Ospedaliera Universitaria 'San Martino', Genova \\ (Dir. Dott.ssa A. Santelli)
}

Introduction: Surgical treatment of craniopharyngiomas still represents a great challenge also for skilled neurosurgeons. When feasible, radical removal must be the goal of surgery. Nevertheless, such an 'aggressive' attitude, which reduces but not eliminates at all the risk of relapse, has to be counterbalanced by heavy morbidity and even mortality. The transsphenoidal approach has specific indications in the management of craniopharyngiomas. Usually, it is best reserved to patients with preferably cystic extra-arachnoid-infradiaphragmatic tumors with small suprasellar extension. Concerning recurrences, a cystic component is frequently present $(80 \%)$. In these cases, if radical surgery appears not feasible for any reason, a more conservative approach aiming to cyst content drainage with immediate release of mass effect symptoms can be chosen either by the transcranial route (cystoventricolostomy) either by a transsphenoidal approach (cystosphenoidostomy).

Materials: We report the case of a young adult presenting with a recurrent cystic $\mathrm{CPH}$ who underwent 10 years before elsewhere to the radical transcranial removal of a purely solid $\mathrm{CPH}$. At follow-up he presented repeated cystic recurrences treated by desultory transcutaneous aspiration of cystic content by means of an intracystic catheter connected to a subgaleal reservoir. Later on, because of further recurrences he was first treated by intracystic injection of bleomycin, then by interstitial radiotherapy and finally by conventional external irradiation. Because of a further cystic recurrence completely occupying the sphenoid sinus with erosion of the roof of the maxillar sinus, he was addressed to our institution. Reviewing patient's previous history and treatment attempts, in our opinion no other 'classical' procedures were possible. In this view, and relying on our experience, an endoscopic endonasal emptying of the cyst was performed by our colleague ENT surgeon with the creation of a permanent drainage (cysto-sphenoidomaxillostomy) of the cyst content into the maxillar sinus by means of an X-shaped silastic catheter. At present the patient is symptom-free and neuroradiologic follow-up at 30 months showed no signs of relapse.

\section{7 \\ Extensive Fibrous Dysplasia of Skull Base: Case Report \\ D.C. Rossi, A. D'Andrea, F. Stefani, G. Zona \\ U.O. Clinica Neurochirurgica e Neurotraumatologica, Azienda Ospedaliera Universitaria 'San Martino', Genova \\ (Dir. Prof. Renato Spaziante).}

Introduction: Fibrous dysplasia is a skeletal developmental anomaly in which normal medullary bone is replaced by a variable amount of fibrous tissue. It is a nonhereditary disorder of unknown cause. Fibrous dysplasia is described in terms of two major types: monostotic, involving a single bone and polyostotic, having multiple lesions involving multiple bones. The two forms are clinically evident in different ages: in the monostotic form the disease can be silent and diagnosis is made usually after puberty: patients 20 or 30 year-old can be asymptomatic. In the polyostotic form the diagnosis is made usually before puberty. The clinical picture is quite variegated and derives from location of the lesion. Clivus and skull base fibrous dysplasia is a rare and relative unknown entity; correct diagnosis is essential to distinguish between different disease can affect the cranial base and to plan the proper management.

Materials: We present the case of a 31 year-old women admitted to our unit for mild traumatic brain injury. neuroimaging showed consequence of trauma (petrous bone fracture) and, as incidental findings, an extensive alteration of clivus, dorsum sellae, occipital condyles and foraminal part of occipital bone. The patient underwent transsphenoidal biopsy. Pathology showed presence of fibrous tissue with low cellularity between bone trabecular fragments. This pattern was consistent with diagnosis of fibrous dysplasia.

Discussion: Clinical features, neuroradiological presentation and treatment options are discussed. 


\section{8}

\section{Arthrodesis and Arthroplasty in Degenerative Disease of the Cervical Spine. Two Different Philosophies}

\section{O. de Divitiis, D. Solari, L.A. Mazzeo, O.V. Corriero, D. Vecchione, F. Esposito, P. Cappabianca \\ Department of Neurological Sciences, Division of Neurosurgery, Università degli Studi di Napoli Federico II, Naples, Italy}

The process of disc degeneration is complex and involves many alterations of normal physiology, as well as the process of aging goes on. The surgical management of cervical discoartrhosis generally requires the decompression of the nervous structures affected, appropriately accompanied by the insertion of a replacement device. Nowadays there are two kinds of devices: fixed, which warrant surgical block of articulation, i.e. arthrodesis, and mobile, which afford to maintain range of motion, namely arthroplasty. We present our preliminary experience using two kinds of DePuy Spine ${ }^{\circledR}$ devices: Cervical CFRP I/P ${ }^{\circledR}$ cage for vertebral fusion and Discover ${ }^{\circledR}$ Cervical Artificial Disc for vertebral arthroplasty. Over a 17-months period, twenty-three patients underwent an anterior cervical discectomy for the treatment of degenerative disease of the cervical spine (21 onelevel procedures and 2 two-levels). We placed, totally, 20 Cervical CFRP I/P cages, 4 Discover Cervical Artificial Discs and in one patient neither of them. Mean overall follow-up period was 8.7 months. All patients were evaluated by the means of neurological examination, Neck Disability Index (NDI), Nurick Disability Score, modified Japanese Orthopaedic Association Score and radiological screening. On post-operative X-ray, we calculated Cobb angle and considered prosthesis positioning and fusion rate. All patients showed an improvement of clinical symptoms on post-operative evaluations. Actually, it cannot be established what device should be considered the best; the results of our preliminary experience showed that both kind of prosthesis resulted effective; nevertheless, a good clinical and radiological preoperative study of each case is the backbone to choose the correct device.

\section{9}

\section{Endoscope Controlled Cerebello-Pontine Angle Surgery. Preliminary Experience.}

\section{O. de Divitiis, Lucia Giovanna Maria di Somma, A. Messina, I. Esposito, L.M. Cavallo, F. Esposito, P. Cappabianca}

Department of Neurological Sciences, Division of Neurosurgery, Università degli Studi di Napoli Federico II, Naples, Italy

The great variations of neurovascular structures relationships and the narrow surgical corridors in the cerebello-pontine angle still represent a challenging matter in the endoscopic approach to this area. Though, we translated our experience in the endoscopic transsphenoidal surgery in the treatment of lesions located in such a region, focusing mainly on microvascular decompression for trigeminal neuralgia or hemifacial spasm and on epidermoid tumors. The surgical procedures were performed using the endoscope $(2.7 \mathrm{~mm}$ in diameter, $18 \mathrm{~cm}$ in length) as the sole visualizing tool of the surgical filed. Our technique employs two surgeons working in close cooperation: the 'co-pilot', who holds the endoscope, making fine position adjustments, and the 'pilot', who works with both hands to perform the procedure itself under dynamic endoscopic view. During the operation we continuously pass between a panoramic view of the neurovascular structures and the close-up view, as during the dissecting manoeuvres. The endoscope follows the in-and-out movements of the instruments whose tips always remain forward the front lens. Even though our experience is preliminary, we feel that this 'dynamic' endoscopic technique can provide some advantages permitting movements of surgical instruments in all the corners that the endoscope allows to visualize. Furthermore the endoscope, thanks to its wider and variangled view, allows safer manoeuvres in this area rich of relevant neurovascular structures. Critical for the effectiveness of the operation is the perfect tuning between the two surgeons in order to have always the tip of the surgical instruments under direct visual control to avoid complications.

\section{0}

\section{Combined Neuro-Surgical and Thoracic Approach for a Unusual Neurofibroma of the Neck. Surgical Case Report}

\author{
F. Givigliano', R. Gagliardi², L. Benvenuti ${ }^{2}$, R. Amoroso ${ }^{2}$, \\ J. Giorgetti ${ }^{2}$, G.F. Menconi ${ }^{1}$ \\ ${ }^{1} \mathrm{Sez}$ Chir Toracica Ospedale Livorno, UO Neuro-Chirurgia \\ Ospedale Livorno
}

Introduction: Sometimes the evidence of strange lesions in border anatomic districts, needs interaction between different specialists, in order to obtain a complete and safetly resection. We report the case of a neurofibroma localized in the neck with inclusion in the left emithorax, treated with 'one-stage' mixed neuro and thoracic surgery.

Matherials and Methods: A 25-years-old man affected by neurofibromatosis with CT scan evidence of expansive lesion of $9 \mathrm{~cm}$ (max. axis diameter) from the left part of the 3 th cervical vertebra, up to the left pulmonary artery, was referred for surgical treatment. We perform a left cervicotomy and a left anterior minitoracothomy. Lesion was adherent, not infiltrated the lung apex, and it disappeared in the neck behind the 'sterno cleido mastoideo muscle', adherent but not infiltrated the carotid, and brachial nervous plexus.

Discussion: The treatment of lesions within the supra-clavicula fossa with thoracic invasion is well described in literature. This double approach allow us to face up to every possible situation like infiltration of the intrathoracic vessels, or close contact with the brachial nervous plexus.

Results: We underline the importance of using different specialists to approach a complex lesion in a middle district, in order to treat safetly the different structures. 
201

\section{Chordoid Meningioma of Tentorium. Case Report, Surgical Technique and Review of Literature}

\author{
F. Signorelli ${ }^{1}$, G. Iofrida ${ }^{3}$, G. Ferraro ${ }^{3}$, G. Donato ${ }^{2}$, \\ R. Maduri ${ }^{1}$, P. Regolo ${ }^{3}$ \\ ${ }^{1}$ Cattedra e U.O. di Neurochirurgia - Università degli studi \\ di Catanzaro, ${ }^{2}$ Cattedra e U.O. di Neuropatologia - \\ Università degli studi di Catanzaro, ${ }^{3}$ U.O. di Neurochirurgia - \\ Istituto di cura 'Santa Rita' - Milano
}

Introduction: Meningiomas are common tumors of the central nervous system. Their annual incidence is 2.3 in 1,00,000. Chordoid meningioma $(\mathrm{CM})$ is a meningioma containing regions that are histologically similar to chordoma, with trabeculae of eosinophilic, vacuolated cells in a myxoid background and corresponds to WHO grade II. Such tumor is a rare variant first described by Kepes et al. in 1988 in young patients associated with Castleman syndrome. To the best of our knowledge, about 70 cases have been previously published, but unpublished cases are often recorded.

Methods: We present CT scan, MRI, histopathological findings and the surgical technique (video inside) used to perform a sub-total excision of the lesion via a modified subtemporal approach. The tumor was localized in the free border of tentorium with extension in posterior cranial fossa.

Results: We obtain a subtotal removal ( $85 \%$ of the tumor was removed) due to the dense adhesions between the tumor capsule and adjacent neurovascular structures within the posterior cranial fossa. The patient have the total relief of the symptoms. This type of tumor could be associated with an higher growing rate because progesterone-receptor-negative meningiomas tend to be larger than progesterone-receptor-positive tumors, but in our follow-up the tumor is still of the same dimensions of post-operative imaging studies.

Discussion: The histopathological diagnosis of CM is still contradictory. We describe our findings and surgical technique and review the mainly literature. We conclude that this lesion is probably a true subtype of meningioma only in the childhood.

\section{2}

\section{Endoscopic Anatomy of Sylvian Fissure}

R. Maduri, G. lofrida, D. Murrone, F. Signorelli

Chirurgia Neurologica, Catanzaro

Introduction: The endoscopic techniques to reduce brain retraction, vessels, dura and nerves manipulation, need a knowledge of the endoscopic neuroanatomy and surgical maneuvers. The sylvian fissure is a privileged site where the endoscope may be used.

Methods: At the University of Vienna 28 sylvian fissures from cadaver heads, with arteries injected with red silicon through internal carotid arteries, were studied. A pterional craniotomy was performed, exposing the sylvian fissure. After opening the proximal part of the sylvian fissure and retracting the pars triangularis of the third frontal gyrus rigid endoscopes (Karl Storz, Germany) were introduced to enter the deep part of the fissure.
Results: Introducing the endoscopes parallel to a retractor placed over the pars triangularis of the third frontal gyrus, without opening widely the arachnoid of the sylvian fissure or retracting the temporal lobe, it was possible to see the middle cerebral artery and its relathionships with important surgical landmarks. The origin and course of all M1, M2 and M3 sylvian branches, including lenticulostiate arteries, were identified.

Conclusion: The endoscopic view allowed full visualization of an intact anatomy for the sylvian fissure and could be an important adjunct in neurosurgical interventions performed in this area, reducing the manipulations and providing a thorough vision of hidden corners.

\section{3}

\section{Minimally Invasive Treatment for Neorogenic Intermittent Claudication: X-STOP Device in Our Experience}

\section{R. Maduri, D. Murrone, G. lofrida, F. Signorelli}

\section{Chirurgia Neurologica, Catanzaro}

Introduction: Neurogenic intermittent claudication (NIC) is a symptom associated with lumbar spinal stenosis (LSS). Decompressive surgery (laminectomy) is the 'gold standard' treatment for NIC. X-Stop (St. Francis Med.) is a minimally invasive interspinous implant that reduces extension at the symptomatic level but allows flexion and axial rotation. It could be an alternative to conservative or invasive treatments in selected patients with LSS.

Methods: 25 patients with LSS (mean age of 71 years, range 37-70 years) were implanted with X-STOP. They were assessed preoperatively by pain evaluation, clinical and radiological exams. 10 patients had disc erniation, facet joints arthrosis and MODIC I degeneration of intervertebral bodies; 14 patients had concentric lumbar spine stenosis associated in 6 patients with a GRADE I degenerative spondilolisthesis. All patients had leg pain with paresthesias, CNI and moderate motor deficits. X-STOP was placed between the spinous processes by making an incision of about $3 \mathrm{~cm}$, while preserving the interspinous ligament.

Results: No patients had surgical complications or implant dislocation. The clinical assessment found an improvement of the algic symptomathology. Patients were dimissed after 2 or 3 days.

Discussion: X-STOP is a new surgical approach to LSS and could be indicated for selected patients that do not get benefit from conservative procedures. 


\section{Oncologic Surgery}

\section{4 \\ Soluble E-Cadherin Level in Gastric Cancer Patients \\ S. Caruso, C. Pedrazzani, D. Marrelli, A. Neri, G. Corso, \\ A. Berardi, G. De Marco, M.E. Perrotta, F. Roviello \\ U.O. Chirurgia Oncologica, Università degli Studi di Siena}

Introduction: Elevation of E-cadherin serum levels has been reported in several tumors. The aim of this study was to evaluate the role of soluble E-cadherin in gastric cancer patients.

Methods: Concentrations of soluble E-cadherin from 124 gastric cancer patients and 45 controls were analyzed.

Results: All patients were prospectively studied with a median follow-up of 38 months. Soluble E-cadherin levels were not statistically different between gastric cancer patients and control subjects $(p=0.060)$. A low sensitivity and specificity were estimated by the ROC curve offsetting its utility as a diagnostic marker. Soluble E-cadherin levels did not correlate with survival and no significant elevation was noticed according to the different clinicopathological characteristics, if not in relation to the age $(p=0.008)$ or the presence of systemic metastases $(p=0.044)$. Conversely, statistically significant differences in 5-year survival rates were observed according to the tumour site $(\mathrm{p}=0.013)$, the type of surgery $(\mathrm{p}<0.001), \mathrm{pT}$ $(\mathrm{p}<0.001), \mathrm{pN}(\mathrm{p}<0.001)$ and $\mathrm{M}(\mathrm{p}<0.001)$ categories. The correlation between soluble $\mathrm{E}$ cadherin and age was confirmed by linear regression analysis both for gastric cancer patients $(p=0.015)$ and control subjects $(\mathrm{p}=0.030)$.

Discussion: Soluble E-cadherins did not seem useful in gastric cancer patients. The correlation with age claims for age matched comparisons.

\section{5}

\section{Pathology Characteristics that Optimize Outcome Prediction of T1NOMO Breast Cancer}

S. Caruso, G. De Marco, M.E. Perrotta, A. Neri, D. Marrelli, C. Pedrazzani, G. Corso, G. Cerullo, Y. Lambert,

M. Di Martino, F. Bettarini, B. Rampone, F. Roviello

U.O. Chirurgia Oncologica, Università degli Studi di Siena

Introduction: A series of T1N0Mx breast cancer was analyzed to identify features that may predict outcome and influence treatment strategy.

Methods: Data from 621 female patients with T1N0M0 breast carcinoma with a median follow-up of 7 years were analyzed.

Results: The majority of the carcinomas were ductal type (78.9\%). The greater part $(52.7 \%)$ of primary lesion was classified as moderate grade (G2), while $20.9 \%$ as G1 and $14.8 \%$ G3. Peritumoral lymphatic/blood vessel invasion was detected in $81(13 \%)$ tumors.
The strongest single feature influencing overall survival was LVI $(\mathrm{p}=0.0007)$. At multivariate analysis statistically significant prognostic features were also age $<60(\mathrm{p}=0.008)$, premenopausal status $(p=0.017)$, immunohistochemically detected positivity of estrogen hormone receptors $(E R)(p=0.001)$, Mib1 $(p=0.004)$ and BCL2 $(\mathrm{p}=0.050)$.

Discussion: Identifying aggressive disease in T1N0M0 breast cancer would give valuable information for planning adjuvant therapy. Our analysis revealed that presence of LVI is the most reliable indicators of unfavourable outcome in T1N0M0 breast cancer. In addition, high Mib1 and BCL2 immunopositivity and low ER immunopositivity also have predictive value. The risk in women aged 60 years or younger was greater than that in women aged older than 60 years. No statistical difference was found between smaller and larger tumors $(\mathrm{pT}, \mathrm{p}=0.46)$

\section{6 \\ Laparoscopic Treatment of Gastric GIST: Report of 21 Cases and Literature's Review}

\author{
F. Catena, M. Di Battista, P. Fusaroli, L. Ansaloni, \\ V. Di Scioscio, D. Santini, M. Pantaleo, G. Biasco, \\ G. Caletti, S. Saveri, F. Coccolini, F. Gazzotti, L. D'Alessandro, \\ A. Pinna \\ Policlinico S. Orsola-Malpighi U.O. Chirurgia d'Urgenza, \\ Bologna
}

Background: Although the feasibility of laparoscopic resection of gastric GIST has been established, various aspects are debated. This paper describes the problems of minimally invasive resection of gastric GIST and compares this experience with an extensive literature review.

Study Design: Between August 2001 and December 2006, 21 consecutive patients undergoing laparoscopic resection of gastric GIST were enrolled in a prospective study. A literature's review of laparoscopic treatment was performed on Pubmed using key-words GIST, surgery. A comparison with Author's experience with open wedge resection of GIST tumors (25 cases from November 1995 to December 2000) was also carried out. Stastical analysis was based on chi square/Fisher exact test and t-Student evaluation.

Results: Twenty-one patients, mean age 50.1 years (range, 34-68 years), were submitted to laparoscopic wedge-segmental gastric resections. Mean tumor size was $4.5 \mathrm{~cm}$ (range, $2.0-8.5 \mathrm{~cm}$ ). Mean operative time was 151 minutes (range, 52-310 minutes), the mean blood loss was $101 \mathrm{ml}$ (range, $10-250 \mathrm{ml}$ ), and the mean hospital stay was 4.8 days (range 3-7 days). There were no major operative complications or mortalities. All lesions had negative resection margins. At a mean follow-up of 35 months, all patients were disease free. Morbidity, mortality, length of stay and oncologic outcomes were comparable to the open surgery retrospective evaluation. $(p=n . s$.

Conclusions: As found also in literature review the laparoscopic resection is safe and effective in treating gastric GISTs. Given these findings as well as the advantages afforded by laparoscopic surgery a minimally invasive approach should be the preferred surgical treatment in patients with small- and medium-sized gastric GIST. 
207

\section{Surgical Treatment of Duodenal GIST}

L. Leone, A. Di Cintio, S. Ghinassi, B. Battaglia, M. Catarci, G.B. Grassi

U.O.C. Chirurgia Generale Oncologica, A.C.O. San Filippo Neri, Roma

Introduction: GIST are mesenchymal neoplasies that interesting gastrointestinal feature. Their incidence in the last ten years is little by little increased thanks to the coming and to the perfectioning of new methodical (immunoistochimical, electron microscopy) what some have allowed to classify as a GIST, some neoplasies before known like leiomyosarcoma or tumor neural origin. Ad today, the Gist represent $1-5 \%$ of all the neoplasias and they discover more frequently over the 50 years old. The duodenal Gist are, about, 3-5\% of all the tumors of gastrointestinal feature. The most tumours are localizated in the second or third duodenum portion. The most duodenal GIST are benign, but in 30 case \% give metastasis, rare on the road lymphatic, mostly on the road haematic and more frequently to the liver. It's difficult to understand their potential malignity and prognosis. The surgical objective must be to obtain a R0, with free neoplasia resection margins. In fact the survival appears to be in parallel with the complete surgical treatment.

Patients and Methods: We have considerated, in the period 2003-2007, 19 patients affected with GIST and submitted to surgical operation, 2 of such were patients affected with duodenal GIST.

Results: Being also rare neoplasias, the duodenal GIST are whats between all the types of GIST have the most difficult surgical approach: in fact because of the anatomic position what the duodenum occupies, to obtain the complete oncological treatment it is necessary to turn to radical surgery with elevated mortality intra e peri operative and many post operative complication. In the our case histories, such pathology has been treated taking into consideration the most recent updatings surgical and with the neoadiuvante therapy help.

Conclusions: In the last few years a series of studies has been concentrated on the possibility of reducing the neoplastic mass to be able to practise instead of a DCP, a duodenal partial resection. In selected cases, how the Kurihara et al. study shows. That has made himself possible through embolization of the duodenal artery which has reduced the dimensions of the tumor. Bigger attention has been concentrated on the use as chemotherapeutic of imatinib mesilato which, as neoadiuvante therapy, could reduce the tumour dimensions and allow a duodenal resection instead of several demolition surgery.
208

\section{Clinical Outcome of 267 Patients Undergoing Early Post-Operative Enteral Feeding through a Fine Needle Catheter Jejustomy (FNCJ) after Upper Gastro- Intestinal Oncology Surgery}

\author{
S. Pozzi ${ }^{1}$, F. Luca ${ }^{1}$, S. Cenciarelli' ${ }^{1}$ C. Grassi ${ }^{1}$, B. Andreoni ${ }^{2}$, \\ R. Biffi \\ ${ }^{1}$ Division of Abdomino-Pelvic Surgery-European Institute of \\ Oncology-Milan-Italy; ${ }^{2}$ Division of General and \\ Laparoscopic surgery-European Institute of Oncology- \\ Milan-Italy
}

Introduction: Enteral nutrition has gained considerable popularity due to its favourable effects on the digestive tract and its lower cost and rate of complications compared to parenteral nutrition. Some studies reported a high rate of complications related to implant and use of FNCJ. Purpose of this study was to evaluate early and late complications of FNCJ in a consecutive series of 267 oncology patients undergoing early postoperative enteral feeding after surgery.

Methods: Patients underwent FNCJ implantation according to Delanys's method at the end of their scheduled surgical procedure. EN program was started on postoperative day 1 in the surgical ICU or in the ward with $15 \mathrm{ml} / \mathrm{h}$ and progressed to the optimal goal of $80 \mathrm{ml} / \mathrm{h}$ by postoperative day 5 . A polymeric nutritionally complete diet was used.

Results: FNCJ was always removed in the outpatient clinic after hospital discharge (mean duration of use was 18 days/SD). One case of tube blockage and two cases of limited surgical site infection have been observed as complications in this series. No long-term complications have been detected after a mean follow-up of 13 months (range 0.5-75).

Discussion: Routine use of FNCJ in malnourished patients undergoing major surgical procedures on upper gastrointestinal tract is safe and effective in providing nutritional support.

\section{9}

Outcome after TT and LT Coloanal Anastomosis in Low Anterior Resection for Rectal Cancer

B. Battaglia, A. Belardi, L.M. Cosentino, D.R. Ferrante, M. Catarci, A. Di Cintio, L. Leone, G.B. Grassi

Department of General and Oncological Surgery, S.

Filippo Neri Hospital, Rome, Italy, Via Martinotti 20

Introductions: The purpose of this study was to compare the termino-terminal and latero-terminal coloanal anstomosis after low anterior resection for rectal cancer with regard to functional and surgical outcome and to all complications well known of these techniques.

Methods: This retrospective study included 655 patients with rectal cancer undergoing total mesorectal excision and coloanal anastomosis were randomized to receive either a termino-terminal (263 patients) or latero-terminal anastomosis (392) using the descending 
colon. Patient characteristicss in both group were very similar regarding age, gender, tumor level. All operations were performed by a uniform technique and by the same surgical group. Surgical results and complications were recorded. Patients were follwed with a functional evaluation at 48 months postoperatively.

Results: There was not so significant difference in surgical outcome between the two techniques with respect to postoperative complications: anastomotic dehiscence $(2.5 \% \mathrm{TT}, 1.3 \% \mathrm{LT})$, fistulas (3.1\%TT, $1.4 \% \mathrm{LT})$, infections $(2.6 \% \mathrm{TT}, 3.3 \% \mathrm{LT})$, reoperation, stenosis $(2.8 \% \mathrm{TT}, 1.6 \% \mathrm{LT})$, subocclusion $(1.4 \% \mathrm{TT}, 2.1 \% \mathrm{LT})$ and mortality (both $0 \%$ ); comparing functional results in the two study group, only bowel function (incontinente, constipation, sensation of incomplete evacuation, etc.) reached a significant difference in favor of the latero-terminal anastomosis.

Conclusions: The data from this study show that either anastomosis performed on the descending colon in low anterior resection with total mesorectal excision are methods that can be used with similar expected functional and surgical results. The last choice is up to the surgeon and its abilities.

\section{0 \\ Implementation of D2 Lymph Node Dissection in a Non-Dedicated Western Center \\ S. Ghinassi, M. Catarci, L. Montemurro, S. Mancini, G. Ricci, A. Di Cintio, L. Leone, G.B. Grassi \\ Department of General and Oncological Surgery, San Filippo Neri Hospital, Roma}

Introduction: At present, the role of lymph node dissection for gastric cancer is far from being solved; what level of lymphadenectomy is indicated in not specialised western centres and not at high volume of cases? Our aim is to analyse the impact on prospectively morbidity, mortality, relapse and survival has adoption of a protocol of lymphadenectomy extended in a western centre not dedicated.

Materials and Methods: We have considerated, in the period 2000-2006, 145 patients with gastric cancer submitted to surgical operation with average age of 66.3 years. Have been carried out a total of 104 subtotal gastrectomy and 41 total gastectomy, lymphadenectomy D1 $\alpha$ in 33 cases and D2 in the remaning 112 .

Results: At the time of censor (follow-up average 47.6 months) the average survival was 39.3 months, with a rate to 5 years of $51.3 \%$. The average time to relapse of disease was equal to 35.3 months, with a rate to 5 years equal to $42.7 \%$. The morbidity and mortality are not related to any of the variables taken into account and in particular to the type of neither lymph node dissection or extension of resection, there was, moreover, no statistically significant difference as regards the survival and the rate of relapse than the two types of compared lymphadenectomies.

Conclusions: In conclusion the analysis of this prospective indicates that the adoption of a protocol of lymphadenectomy extended in a surgical centre western dedicated is possible and does not increase morbidity and mortality.
211

\section{Spleen Preserving Distal Pancreatectomy for} Pancreatic Metastasis of Breast Cancer

L. Leone, M. Catarci, A. Belardi, A. Di Gregorio,

A. Torcasio, S. Ghinassi, A. Di Cintio, G. Longo, G.B. Grassi

Department of General and Oncological Surgery,

San Filippo Neri Hospital, Rome

Introduction: The video shows the case of a 53 y.o. female patient submitted to left mastectomy for an invasive lobular carcinoma seven years before.

Materials and Methods: One year before she was also submitted to external beam radiation therapy for mediastinal relapse of the disease with complete response. Up to one month before complete negative follow-up. A new raise in serum markers prompted a PETCT scan that identified a positive area in the pancreatic tail.

Results: The patient underwent a spleen preserving distal pancreatectomy. Actually (1 years after) she is alive and without disease.

Discussion: Surgical approach in these patients is discussed. Lobular breast carcinoma is an aggressive kind of tumor and an intraperitoneal single metastasis could be treat by surgeon.

\section{2}

\section{Anal Malignancies Associated to Condyloma Acuminata}

\author{
M. Mistrangelo4, P. Cassoni ${ }^{1}$, I. Castellano ${ }^{1}$, I. Dal Conte ${ }^{2}$, \\ M. Chiriotto ${ }^{2}$, S. Delmonte ${ }^{3}$, S. Rondoletti ${ }^{3}$, G. C. Serra ${ }^{5}$, \\ A. Mussa ${ }^{5}$
}

${ }^{1}$ Anatomo-Patological Department, University of Turin, Italy, Dir. Prof. G. Bussolati, ${ }^{2}$ nffectivous Diseases Department, University of Turin, Italy, Dir. Prof. Di Perri, ${ }^{3}$ Dermatological Department, University of Turin, Dir. Prof. M.G. Bernengo,

${ }^{4}$ General and Emergency Surgery II, University of Turin, Dir Prof. M. Morino, ${ }^{5}$ Surgical and Oncological Department, University of Turin, Italy, Dir. Prof. A. Mussa

Introduction: Human Papillomavirus (HPV) infection is emerging as an important factor in the oncogenesis of squamous cancers. The incidence of HPV-associated anal cancer has recently increased, largerly attribuited to immunocompromised states.

Patients and Methods: 807 patients (513 M and $294 \mathrm{~F}$ ) were affected by anal and perianal condylomata in a period from October 1999 and September 2006. $11.5 \%$ of patients were HIV+, $7.1 \%$ $\mathrm{HBV}+$ and $2.9 \% \mathrm{HCV}+.605$ patients were submitted to surgery. Results: Histological exam revealed a degeneration in 26 patients (4.3\%). 14 of these patients were HIV $+(53.8 \%$ of degenerations); and 2 patients were immunosuppressed (7.7\%). Histological exam: 9 Buschke Lowenstein neoplasms, 10 Bowen disease or Bowenoid papillomatosis; 2 carcinomas in situ; 1 high displasia; 1 basocellular carcinoma and 3 squamous carcinomas. HPV tipization was performed in 135 patients: 18 high risk and 12 low/high risk. Patients with diagnosis of Buschke Lowenstein tumour was sumbitted to radical surgery; patients with anal carcinoma to RT + CT or to surgical excision; and other patients to surgical excision. All patients are actually in follow up. 
Conclusions: Histopatological examination in anal warts should be performed to confirm the diagnosis and to detect precancerous or cancerous changes. An accurate follow up is mandatory to prevent transformation in anal carcinoma.

213

\section{Sentinel Node Biopsy in Patients Affected by Anal Carcinoma}

M. Mistrangelo ${ }^{5}$, A. Mobiglia ${ }^{6}$, M. Bellò ${ }^{1}$, P. Cassoni, G. Beltramo ${ }^{3}$, E. Milanesi', A. Mussa ${ }^{6}$

${ }^{1}$ Nuclear Medicine Department, University of Turin, Dir. Prof. G. Bisi, ${ }^{2}$ Anatomo-Pathological Department, University of Turin, Dir. Prof. G. Bussolati, ${ }^{3}$ Department of Radiotherapy, University of Turin, Dir. Prof. U. Ricardi, ${ }^{4}$ C.U.R.O., University of Turin, Dir. Dottssa P. Racca, ${ }^{5}$ General and Emergency Surgery II, University of Turin, Dir Prof. M. Morino, ${ }^{6}$ Oncological Surgical Department, University of Turin, Dir. Prof. A. Mussa

Introduction: Inguinal metastasis have been established as an independent prognostic factor for local failure and overall mortality. We applied the sentinel node biopsy in anal cancer.

Methods: 35 patients studied between October 2001 and December 2006. A informed consent was signed. The patients were injected with $37 \mathrm{MBq}$ of nanocoll-Tc-99 $\mathrm{m}$ in the four cardinal points around the neoplasm. Patients were submitted to inguinal sentinel node biopsy in operating theatre. Intraoperative localization of sentinel node was performed with a Scintiprobe MR 100, Politech ${ }^{\circledR}$, Carsoli Italy.

Results: Detection rate was $97.1 \%$. Postoperative lymphorrea occurred in 20 patients $(57.1 \%)$. Metastases were found in 7 patients $(20 \%)$, in 2 cases were bilateral. Patients affected by inguinal metastases were treated also with inguinal radiotherapy. Median follow up was 21.44 months (range 2-53 months). 7 patients died (20\%). 5 of the 7 patients died were N2. 8 patients recurred (22.5\%): 6 after RT$\mathrm{CT}$ and 2 patients after surgery. Other patients were disease free. Up to now we have not observed any inguinal metastases in patients negative at the first examination.

Discussion: In order to evaluate the state of inguinal nodes the sentinel node technique was applied in patients with anal cancer. Inguinal radiotherapy was performed only in N2 patients avoiding the morbidity related to this procedure in $\mathrm{N} 0-\mathrm{N} 1$

\section{4}

Large Bowel Auto-Amputation and Passage of a Colon 'Cast' after Left Hemicolectomy

\author{
A. Biondi, R. Persiani, S. Rausei, F.C.M. Cananzi, \\ M. Zoccali, D. D'Ugo \\ Istituto di Patologia Speciale Chirurgica-Policlinico A. \\ Gemelli, Roma
}

Introduction: The anal passage of a cast of the large bowel caused by ischemic injury has been reported only rarely in literature. We encountered this unusual complication after left hemicolectomy.
Methods: Clinical, radiographic, pathologic, and endoscopic findings were obtained from the medical records, and the literature was searched for similar cases.

Results: A 52-year-old male underwent a left hemicolectomy for colon cancer with inferior mesenteric artery ligation. Thirty seven days after a $15-\mathrm{cm}$ full-thickness infarcted colon segment passed per anum. Under radiologic and endoscopic evaluation, the granulation tissue conduit connecting the remaining viable bowel was visualized and was enoscopically dilated. Because of impelling signs of obstruction the patient required a laparotomy to resect the stricture and restore bowel continuity. A literature review revealed nine cases of passage of a large bowel cast following aneurysm surgery but also after colorectal surgery.

Discussion: The spontaneous passage of a full-thickness colon cast is a rare manifestation of acute colonic ischemia. This phenomenon is reported in association with aneurysm surgery but also in the postoperative course of colorectal surgery when IMA is ligated. In these cases, even if conservative treatment is feasible, the definitive treatment is surgical with favourable outcome.

\section{5}

\section{Surgical Treatment of Gastric Cancer and its Post-Operative Morbidity and Mortality}

S. Rausei, R. Persiani, A. Biondi, F.C.M. Cananzi, M. Zoccali, D. D'Ugo

Istituto di Patologia Speciale Chirurgica-Policlinico A. Gemelli, Roma

Introduction: The occurrence of early surgical complications after gastrectomy as a treatment for gastric cancer has been reported to have a negative impact on long-term survival. Aim of this study was to identify treatment-related factors that can predict morbidity and mortality in gastric cancer patients undergoing surgery.

Methods: 388 patients who underwent operations for gastric cancer were reviewed and grouped according to the type of surgical treatment. The endpoints were postoperative morbidity, mortality and the length of hospital stay after surgery.

Results: Overall morbidity and mortality rates were 2.3 per cent and 16.2 per cent respectively. Morbidity rates were higher in patients over 64 years of age, when gastric tumor was resected along with the spleen and when extended lymphadenectomy was performed. After subtotal gastrectomy, the type of reconstructive surgery performed was not associated with morbidity. Patients older than 64 years of age had longer postoperative hospital stays, whereas Roux-en-Y gastrojejunostomy was predictive of a shorter stay. Mortality was not influenced by any surgically related factors.

Discussion: Age, splenectomy and extended lymphadenectomy were independently associated with the development of complications after gastric cancer surgery. After subtotal gastrectomy, Roux-en-Y gastrojejunostomy was associated with a short postoperative length of stay. 


\section{6}

cDNA Microarray Analysis in Gastric Cancer: Towards the Prediction of Lymph Node Status?

F.C.M. Cananzi, R. Persiani, S. Rausei, A. Biondi, M. Zoccali, D. D'Ugo

Istituto di Patologia Speciale Chirurgica-Policlinico A. Gemelli, Roma

Introduction: Aim of this study is to investigate whether gene expression analysis by microarrays (MA) technology can be used to predict lymph node status in gastric cancer (GC).

Methods: 32 GC patients who underwent gastrectomy. Gene expression profiles were obtained by cDNA MA on primary tumor specimens of 10 patients. Only genes with a evident flag in at least 6 samples were further studied. The remaining data were submitted to Volcano-plot t-test, categorized using 'Gene Ontology' and then verified by quantitative real-time PCR on the specimens of all 32 patients.

Results: $22 \mathrm{pN}+$ and $8 \mathrm{pN} 0$ patients were identified $(6 \mathrm{pN}+$ and 4 pN0 in MA analysis sample). The cDNA MA analysis identified 35 genes differently expressed in the two groups. By biological categorization, 4 proteins were associated with metastatic lymph nodes: Claudin 4, Matrix Metalloproteinase 2, TGFbeta, Chemokine ligand 21 . The Claudin 4 levels were significantly lower in patients with metastastic nodes, whereas the levels of Matrix Metalloproteinase 2, TGFbeta and Chemokine ligand 21 were significantly higher. When tested by PCR, only Claudin 4 was differentially expressed with statistical significance in the two groups.

Discussion: MA analysis, identifying genes associated with lymph node metastasis, could improve the selection of patients with advanced GC for extended lymph node dissection, multimodal therapy or alternative strategies.

\section{7 \\ Radiofrequency Ablation (RFA) of Liver Tumors: Our Experience}

\section{Barlettai, F. Scassa, F. Filidei, S. Panicucci, C. Bagnato,} P.V. Lippolis

U.O. Chirurgia Generale e d'Urgenza, Direttore

Prof. M. Seccia - Azienda Ospedaliera Universitaria Pisana

Introduction: Primary and secondary malignant hepatic tumors (MHT) are some of the most common tumors observed. Surgical resection is the definitive curative option, but not for all patients. In patients not eligible for liver resection, recent studies indicate that minimally invasive techniques, as radiofrequency ablation (RFA), are effective for treating MHT.

Methods: Between January 2003 and July 2007, 36 patients with primary or secondary MHT were treated with RFA. 78 procedures were carried out: 5 laparoscopic, 27 open and 46 percutaneous. Ten lesions were HCC and 68 metastases: 52 colorectal cancers, 4 gastric cancers, 6 undifferentiated retroperitoneal tumors, 4 breast cancers, 1 lung cancer and 1 gastrinoma.
Results: One patient died in the first postoperative day for cardiac failure and one developed pneumotorax during the needle deployment. The mean length of stay was 5.7 days ( 3 for percutaneous, 9 for laparoscopic and 10.6 for open procedures). During the follow-up study, 10 patients died for disease's progression, 25 are still alive, but 3 of them showed a cancer recurrence.

Discussion: RFA is safe, cost effective and offers a good local tumor control. In comparison with the natural or post-chemotherapy neoplastic course, RFA demonstrated to be able to improve expected overall survival rate.

\section{8 \\ Our Experience in Multimodal Approach in the Treatment of Advanced Gastric Cancer

\author{
F. Tauceri ${ }^{1}$, G. Mura ${ }^{1}$, A. Moretti ${ }^{2}$, M. Framarini ${ }^{1}$, \\ A. Vagliasindi 1 , F. Matteucci ${ }^{2}$, D. Cavaliere1, R. Galassi², \\ G.M. Verdecchia ${ }^{1}$ \\ ${ }^{1}$ Division of Surgery and Advanced Cancer Therapie, \\ Morgagni Hospital, Forlì, Italy; ${ }^{2}$ Nuclear Medicine and \\ Radiology - Morgagni Hospital - Forlì, Italy
}

Introduction: Advanced gastric cancer has still poor prognosis. A promising upward trend is showed in last decades, related to the diffusion of extended nodal dissection. Advanced gastric tumors are nowadays increasingly approached with multimodal strategies.

Methods: 78 patients were operated on for gastric cancer in the period March 2005-June 2007. Forty-one presented advanced stage disease. 16 patients with clinical diagnosis of T3-T4, confirmed by laparoscopy, without extra-abdominal metastasis and FDG-PET positive at baseline, were enrolled in present study. All such patients were sent to neoadjuvant chemotherapy (4 cycles PELF-schedule). In 3 patients chemotherapy was stopped for fast progression of disease, in one for toxicity. One patient, when completed the chemo treatment, refused surgery. All these patients died soon. Three patients are still in treatment. The remaining 8 patients presented a reduction of glucose uptake at mid-treatment FDG-PET, so classified as 'metabolic responders' and underwent D2-D3 gastrectomy with complete debulking ( $\mathrm{R} 0$; $\mathrm{CC}$ score $=0$ ), followed by sixty-minutes hyperthermic peritoneal chemotherapy (HIPEC) at $41.5^{\circ}$.

Results: The pathological evaluation of the response to chemotherapy described five cases of total/subtotal regression (Becker's grade 1) and three case of partial regression (grade 2). Medium follow-up is of 245 days: one patients died for peritoneal carcinosis after 10 months; the rest of patients are alive and disease-free.

Discussion: Neoadjuvant chemotherapy could improve both resectability and survival. HIPEC can reduce the local and peritoneal recurrences. Our preliminary data outline the role of FDG-PET in early prediction of response to neoadjuvant chemotherapy in patients with advanced gastric cancer. $\overline{134} \quad \overline{\text { Eur Surg Res 2008;40:61-183 }}$ 20th National Congress of the Italian Polyspecialist Society of Young Surgeons (IPSYS) 


\section{9}

\section{Surgery Associated with Adjuvant Therapy in the Treatment of Stage IV Melanoma: Experience in 76 Patients}

\author{
F. Tauceri, G. Mura, M. Framarini, A. Vagliasindi, \\ D. Cavaliere, G.M. Verdecchia \\ Division of Surgery and Advanced Cancer Therapies, \\ Forlì - Italy
}

Introduction: Survival rates of patients with stage IV melanoma are poor: median survival is $7-8$ months, actual 5 -year survival rates of $5 \%$. Most patients with metastatic melanoma are not able to undergo to resection and are sent to adjuvant therapy.

Methods: 76 patients operated on for IV stage melanoma from January 1995 to June 2007 were evaluated. The disease-free interval, the site and number of first and next metastases, surgery, adjuvant therapies were evaluated. $60 \%$ of patients were submitted to reiterative surgery (overall 155 operations and 169 surgical procedures). $90.7 \%$ of patients were submitted to adjuvant therapies according to aggressive and reiterative schedules: chemotherapy, immunotherapy, dendritic cells vaccine, infusion of Tumor Infiltrating Lymphocytes (TIL), local therapy as electro-chemotherapy.

Results: The overall survival (Kaplan Meier) was 56.7 months, with a 1,3 and 5 year survival of $72.1 \%, 46.5 \%$ e $23.16 \%$ respectively. Reiterative surgery showed significatively higher survival (log rank $4.67, \mathrm{p}=0.03$ ) than one-stage surgery. Multivariated analysis for $>36$ months free interval, reiterative surgery, adjuvant therapies, site of metastasis according to AJCC was performed: reiterative surgery was shown as indipendent prognostic factor $(p<0.05)$.

Discussion: Our series shows that resection of metastases improves overall survival and isn't related with site and number of metastases. Surgical resection, associated with adjuvant therapies, is an important component to be considered in the treatment of metastatic melanoma. Cytoreductive surgery is to be considered a form of immunotherapy, with efficacy dependent upon the host's ability to control subclinical micrometastases with a sufficient immunologic response.

\section{0 \\ Our Preliminary Experience in Surgery of Peritoneal Carcinomatosis: 161 Cases \\ F. Tauceri, G. Mura, M. Framarini, A. Vagliasindi, D. Cavaliere, G. Giorgetti, G. Solfrini, G.M. Verdecchia \\ Division of Surgery and Advanced Cancer Therapies, Morgagni Hospital, Forlì, Italy}

Introduction: Peritoneal carcinomatosis (PC) is the first cause of death after surgery for abdominal cancers. In selected patients an aggressive cytoriductive surgery combined with Hyperthermic Intraperitoneal Chemotherapy (HIPEC) may improve the mediumlong term survival.

Methods: In the period March 2004-July 2007, 161 surgical procedures (145 elective, 15 in urgency) with diagnosis of PC were performed in one hundred-twenty-eight patients at Division of Surgery and Advanced Cancer Therapies of Forlì. 38 cases were submitted to staging laparoscopy with sampling for biopsy and chemo-sensitivity and resistance testing. The mean peritoneal cancer index (PCI) of population was 10.4 (range 2-34). Cytoreduction was complete (CC0 ) in $52.5 \%$, incomplete in $47.5 \%$. Thirty patients $(13 \mathrm{M}, 17 \mathrm{~F}$, mean age 61.6, mean PCI =9.6), after complete cytoreduction were submitted to HIPEC. The intraperitoneal drug schedules have been different according to the origin of PC: gastric (7 cases), colo-rectal (13), ovaric (8), PMP (2). The average surgical time was $606 \mathrm{~min}$ (range 380-765).

Results: The mean overall survival (Kaplan-Meier) was 19.3 months, median 12.3. Complete cytoreduction had better survival than incomplete (mean 31.6 mo vs. 6.1), $\log$ rank 57.17, $\mathrm{p}<0.00001$; the HIPEC group presented longer mean survival vs. no-HIPEC either overall than only-CC-0 groups -, (35.9 mo vs. 13.2 and 26.6 respectively), $\log$ rank 22.51, $\mathrm{p}<0.00001$.

Discussion: According to most Authors, complete cytoreductive surgery for PC has been able to improve prognosis; HIPEC, when indicated and possible, had better results. Individualized treatment based on chemo-sensitivity and resistance testing is an interesting challenge for future.

\section{1 \\ Laparoscopic Staging of Peritoneal Carcinomatosis: Preliminary Experience in 55 Cases}

\section{F. Tauceri, G. Mura, M. Framarini, D. Cavaliere, A. Vagliasindi, G. Solfrini, C. Grassi, F. D'Acapito, G.M. Verdecchia}

Division of Surgery and Advanced Cancer Therapies Morgagni Hospital, Forlì, Italy

Introduction: Laparoscopy has been reported by many Authors as improving the diagnosis and staging of peritoneal carcinomatosis (PC).

Methods: In the period March 2004-July 2007, 55 patients were submitted to staging laparoscopy for peritoneal carcinomatosis o for diagnostic suspect of it. In 38 cases (12 M, 26 F, mean age 64.9) the PC was confirmed; in 7 patients (3 with preoperative suspect and 4 submitted to laparoscopic re-staging after chemotherapy) no PC was found. Laparoscopic staging was performed in further 10 patients to confirm the clinical diagnosis of advanced gastric cancer (T3-T4) FDG-PET positive at baseline, before they were sent to neoadjuvant chemotherapy. The origin of PC was gynaecological (20 cases), gastric ( 7 cases), mammary (4), peritoneal mesothelioma (3), colo-rectal (2), visceral melanoma and gallbladder (1). Average surgical time was $97 \mathrm{~min}$. In last 12 patients with PC, sampling for chemo-sensitivity testing was performed.

Results: No major complications are reported. In $5 / 55$ patients a laparotomic conversion was performed to assure a correct staging. The patients with PC presented a mean peritoneal cancer index of 16.6 (range 1-34). 21/38 patient were sent to chemotherapy after laparoscopy; 26/38 patients have been subsequently operated on: in 8 cases Hyperthermic Intraperitoneal Chemotherapy has been performed.

Discussion: In our experience laparoscopic approach is effective and safe in confirming the diagnosis and in staging PC. It can 
avoid un-necessary open surgery and can permit earlier chemotherapy, when indicated. The possibility of executing chemo-sensitivity and resistance testing is an interesting opportunity for future individualized treatments.

\section{2}

Abdominal Localized Castleman's Disease: A Case Report

F. Tauceri ${ }^{1}$, M. Framarini ${ }^{1}$, G. Mura ${ }^{1}$, F. Calzolari², D. Barone ${ }^{2}$, A. Vagliasindi ${ }^{1}$, A. Dubini ${ }^{3}$, D. Cavaliere ${ }^{1}$, G. Solfrini ${ }^{1}$, G.M. Verdecchia ${ }^{1}$

${ }^{1}$ Division of Surgery and Advanced Cancer Therapies, Morgagni Hospital, Forlì; ${ }^{2}$ Service of Radiology Morgagni Hospital, Forlì; ${ }^{3}$ Service of Pathology Morgagni Hospital, Forlì

Introduction: Benjamin Castleman first described multicentric Castleman's Disease (CD) in a series of case in 1954. Synonyms are: Angiofollicular Lymph Node Hyperplasia, Angiomatous Lymphoid Giant Benign, Lymphoma Hamartoma of the Lymphatics, Giant Lymph Node Hyperplasia. CD is separated into localized or multicentric disease. It is a rare disorder characterized by non-cancerous growth that may develop in the lymph node tissue throughout the body.

Case Report: A case of 19-year-old man is reported, who presented ipogastric and left iliac pain for four months. US-scan detected a mass near iliac vessels and ileo-psoas muscle, suspect for nodal. PET-CT detected in left iliac fossa, a hypermetabolic area (SUV $\max =6.46$ ). Abdomen CT-scan confirmed an oval $47 \times 26 \mathrm{~mm}$ mass with hypothesis of Castleman's Disease. A CT-guided fineneedle sampling of the lesion was performed: pathological evaluation revealed hyaline-vascular type $\mathrm{CD}$. The patient underwent open surgery with radical exeresis of the lesion. The mass, size $7 \times 5 \times 2 \mathrm{~cm}$, lay on ileopsoas muscle, in left iliac fork, between iliac artery and vein. The patient is alive and disease-free after 6 months. No adjuvant therapy was performed after surgery.

Discussion: The abdominal site is rare $(10 \%)$ in localized form of $\mathrm{CD}$. The diagnostic suspect of CD has to be confirmed, when possible, by pre-operative biopsy: it's very important for planning correct therapy. Complete surgical exeresis is indicated only in the localized form. Surgery could only reduce the symptoms in multicentric CD. Multicentric presentation need chemotherapy associated with surgery. In this form relapse occurs in $25 \%$ of cases.

\section{ORL}

223

Malignant External Otitis: Case Report

M. Cavaliere, F. Ricciardiello, R. Grassia, E. Zeccardo, G. Sequino, A. Scarpa e M. lengo

Chirurgia ORL, Policlinico Federico II, Napoli

Introduction: Malignant External Otitis (MEO) is an infection that involves the temporal and adjacent bones; it occurs primarily in immunocompromised people, especially old patients with diabetes mellitus. The most frequent pathogen is Pseudomonas Aeruginosa (98\%). Patients complain of severe otalgia worsening at nigh and otorrhea. Clinical findings include granulation tissue in the external auditory canal, especially at the bone-cartilage junction. Facial and other cranial nerve palsies indicate a poor prognosis.

Case Report: We describe a case of a 79-year man suffering from type II diabetes mellitus, hypertension and previous stroke. Since one month, the patient presented left othorrea and severe temporal-parietal pain complicated in the last days by periferic facial nerve palsy. Under oto-microscope we highlighted granulation tissue in the external auditory canal, especially on the posterior wall at the bone-cartilage junction (histologically: 'general fibro-inflammatory process with acute and xanto-granulomatous aspects'). TC scanning showed a normal pneumatization of middle ear with inflammatory tissue obstructing and damaging mastoid cells. Ear secretion's culture was positive for Pseudomonas Aeruginosa. We had administred parenteral antibiotic therapy for 4 weeks (piperacillina-tazobactam and amikacin) and daily local medications. After this period we performed canal wall down tympanoplasty with facial nerve decompression. Subsequently the patient underwent daily hyperbaric oxygen therapy (twenty applications, lasting $90 \mathrm{~min}$, at 2.5 atmosphere). Two year follow up shows no relapses.

Discussion and Conclusion: MEO represents a very serious ENT interest disease with the untreated mortality rate approaching $50 \%$. According to our experience and the review of recent literarature we suggest the following protocol:

1. Parenteral intravenously antibiotic therapy for almost 4 weeks;

2. Surgical Toilette;

3. Hyperbaric oxygen therapy.

224

Cholesteatoma Microbiology: Clinicans and Therapeutic Reliefs

M. Cavaliere, F. Ricciardiello, G. Sequino, A. Scarpa,

R. Grassia, E. Zeccardo e M. lengo

Chirurgia ORL, Policlinico Federico II, Napoli

Introduction: Cholesteatomatous chronic otitis media is characterized by tendency to determine both extra-either intracranial serious complications. The pathogenic agents more frequently involved are Pseudomonas, Stafilococcus Aureus and Bacteroides. Although the only therapeutic tool is surgery, it is useful an association with an 
aimed antibiotic therapy to reduce riacutizations and complication incidence.

Materials and Methods: We studied ear secretion culture in 300 patients affected by middle ear cholesteatoma.

Results: 292 samples were positive for infection: in these cases $(=100 \%)$ we have observed pure culture in 172 cases $(59 \%)$ and a polymicrobic association in 120 patients $(41 \%)$. The most bacteria involved were: P. Aeruginosa (31.5\%), S. Aureus (19.4\%), Peptostreptococcus (17.4\%), Bacteroides (12.9\%), Clostridium (3.8\%), Fusobacterium (2.9\%), Propionobacterium (1.9\%), E. Coli (1.6\%); K. Pneumoniae $(0.99 \%)$. In 6 cases $(2 \%)$ we have founded a mycotic superinfection (Aspergillus).

Discussion and Conclusion: The treatment of cholesteatoma requires antibiotic therapy according to cultural analysis. We showed that in adults the best farmacologic association is represented by third generation cephalosporin (or carbapenem) and fluorochinolons; in children it is advisable to use third-generation cephalosporine or amoxicillina/clavulanic acid association. According to our experience it is important a topical therapy too: we use topical antibiotics (pefloxacin, tobramicin) after a previous local clening by aspiration and washing with antiseptic substances ( $3 \%$ boric acid water solution). Finally in case of mycotic superinfections it is sufficient to interrupt topical corticosteroid use and to undertake an antimycotic therapy.

\section{5}

\section{Intraoperative Neuromonitoring of the Recurrent Laryngeal Nerve in Thyroid Surgery}

C. Di Lieto, C. Bruzzi, I.J. Fernandez, O. Piccin, O. Cavicchi, U. Caliceti

Ospedale S. orsola - Malpigli

Inroduction: Monitoring of the recurrent laryngeal nerve (RLN) has been claimed in some studies to reduce rates of nerve injury during thyroid surgery compared with anatomical dissection and visual identification of the RLN alone, whereas its role has not been well established. This study evaluates whether RLN injury can be reduced by the application of this technique during thyroidectomy.

Methods: Of 240 RLN that were at risk of injury in 130 consecutive patients who underwent thyroidectomy with the use of neuromonitoring (continuous monitoring with endotracheal electrodes) was compared with that of 1,211 RLN, in 664 patients that were operated by routine identification only. The incidences of RLN paralysis were compared between the 2 groups.

Results: Postoperative palsy was identified in 13 recurrent laryngeal nerves in the group with the use of neuromonitoring (5.4\%). While 36 recurrent laryngeal nerves palsy was identified in the group without the use of neuromonitoring ( $3 \%$ ). There was no significant difference in postoperative, transient, and permanent paralysis rates between the neuromonitoring and control groups. The sensibility, the specificity, the accuracy and Positive Predictive Value and Negative Predictive Value of neuromonitoring were rispectly $66.7,97.8$ e $95.7,66.7,97.8 \%$ compared with neurostimulation that were rispectly $66.7,96.7,95.1,52.8,98.1 \%$.

Discussion: Neuromonitoring of the RLN during thyroid surgery could not be demonstrated to reduce RLN injury significantly, compared with the adoption of routine RLN identification. However, its application can be considered for selected high-risk thyroidectomies.
226

\section{Day-Surgery and One-Day-Surgery in ORL: Our Experience}

\section{A. Esposito, S. Desiderio, M.A. Langone, G. Di Lorenzo,} M. Mesolella, G. Sequino e V. Galli

Chirurgia ORL, Napoli

Introduction: In the last years the requirements to reduce the sanitary costs pushed the assigned of the sectors to clinical services type in Day-Surgery and in One Day Surgery. So it is posible to practice surgery or diagnostic or therapeutic, invasive and not too, procedure, doing under suregical services with a daily cicle, in local or general anaesthesia, staying one overnight. We value the results our experience about Day-Surgery/One-Day-Surgery from May 2006 integrating it with normal recovery using 5 beds.

Methods: We screened 597 patients to undergo on surgery. The surgical service was adenoidectomy, adenotonsillectomy, exsection head and neck benign tumours, head and neck malignant tumours biopsy transtypanics and tympanocentesis drains and cauterizations nasal varixes. The weekly admission provides 20 patients between clinical and surgical admissions (2 sessions surgical).

We offer:

The first access: routine check preoperating, consent informed signature, counselling;

The second access: surgery;

The third access: follow-up post-operation and discharges. It provides access 1 bis for more check.

Results: The activity DS/ODS answers well to the needs of the patients, optimizing the waiting list. The surgical service has the same validity versus the traditional, it increases the working order differing patient' admission.

Discussion: We hope in the future to expand DS/ODS services to others surgery such as: nasal septum and nasal concha surgery.

\section{7}

\section{Rare Recurrent Angiomatoid Fibrous Histiocytoma of the Neck}

\section{Cassano, S. Fania, L. Vigliaroli \\ I.R.C.C.S. 'Casa Sollievo della Sofferenza', San Giovanni Rotondo (FG), Unità Operativa Complessa di Otorinolaringoiatria, Direttore: Dott. Lucio Vigliaroli}

Introduction: Angiomatoid fibrous histiocytoma arising in a major salivary gland is rare. We encountered a case of AFH affecting the parotid gland in patient with an history of exeresis of a fibrous histiocytoma of the scalp and a new recurrence to the right cervical lynphnode after 5 year.

Methods and Results: The patient was a 14-year-old girl diagnosed as having angiomatoid type of $\mathrm{FH}$ after esofacial parotidectomy. Examination of the resected specimen revealed the tumor had not been completely removed. Accordingly treatment consisted in a right selective neck dissection. The examination of the material indicated minor seating of AFH in 2 levels. The patient showed no signs of local recurrence and distant metastasis for 3 years. 
Discussion: In the domain of fibrous histiocytomas the possibility of distant metastasis (lymph nodes and lungs) is well known though only a total of seven cases have been described: these metastasis have arisen with a cellular, aneurismal, atypical histhotype and sometimes after local recurrences.

\section{8}

\section{Results with Titanium Ossicular Reconstruction Prosthesis}

\author{
A. Ranieri ${ }^{1}$, M.W. Rossetti ${ }^{1}$, V. Ranieri ${ }^{2}$, E. Vetrano ${ }^{2}$ \\ ${ }^{1}$ Functional Unity of Otorhinolaryngology, S. Rita Clinic, \\ Atripalda (AV) Italy; ${ }^{2}$ E.N.T. Clinic, Second University of \\ Naples, Italy
}

Introduction: The object of this study is to evaluate the results when using titanium total ossicular replacement prosthesis (TORP) or partial ossicular replacement prosthesis (PORP) in chronic ear disease.

Methods: Fiftyfour ossicular procedures using a titanium TORP (n. 25) or PORP (n. 29) were prerformed. The ossiculoplasty was performed either alone or in combination with other chronic ear surgery. The ages of the subjects ranged from 8 to 72 years, with a mean age of 37; Thirty were female, and 24 were male. Twelve were inserted during primary procedures, 4 during planned second stage procedures, and 38 during revision surgery. The diagnosis was chronic otitis media in all cases.

Results: Average air-bone gap (ABG) improvement was $13 \mathrm{~dB}$ with closure of the ABG to within $20 \mathrm{~dB}$ in $61 \%$ of cases. Hearing results were better for primary versus revision cases.

Discussion: Titanium is a satisfactory material for use in ossicular reconstruction because of its ease of insertion, tissue tolerance, and low rate of extrusion. Caution is advised when selecting candidates for this procedure during revision surgery, especially if the canal wall and stapes superstructure are absent.

229

\section{Endoscopic Transsphenoidal Surgery - A Multidisciplinary Approach. The Importance of Pre-Surgical Training in Models and in the Anatomy Lab}

\author{
M. Rigante ${ }^{3}$, F. Doglietto ${ }^{1}$, E. De Corso ${ }^{3}$, G.C. Passali3, \\ N. Montano', P. De Bonis', M. Tschabitscher ${ }^{2}$, G. Maira', \\ G. Paludetti ${ }^{3}$ \\ ${ }^{1}$ Institute of Otolaryngology and Neurosurgery - Catholic \\ University of Sacred Heart, Policlinico 'A. Gemelli'- Rome - \\ Italy; ${ }^{2}$ Department of Anatomy, Medical University of Wien \\ - Austria
}

Introduction: Endoscopic transsphenoidal surgery is a recent multidisciplinary approach, in which ENT and neurosurgeons need not to share only specific theoretical knowledge but also train to get acquainted to working with a new technology and together.
Methods: We conceived a model for the initial training of teams made of two surgeons who were asked to simulate a 'four hands' transsphenoidal approach. The timing and accuracy of the teams were measured at different training times. The endoscopic transsphenoidal approach was also performed in fresh frozen heads with the arterial system injected with red latex, at the Laboratory of Endoscopic and Microscopic Neuroanatomy of the Department of Anatomy of Wien University, directed by Prof M. Tschabitscher.

Results: All surgical teams showed a significant improvement in timing and accuracy during their training. The anatomical study was fundamental to become practically acquainted with the complex multidisciplinary anatomy which needs to be mastered before entering the O.R. All the fundamental surgical steps were documented.

Conclusion: The use of models appears useful to get confidence in working with a technology (i.e. endoscopy) that is new to the neurosurgeon but also for ENT surgeons in traning. The anatomical dissection laboratory is a fundamental step for the multidisciplinary team before entering the O.R.

\section{Orthopedic Surgery}

\section{0 \\ External Fixation for Pediatric Shaft Humeral Fractures}

N. Catena, D. Pontoriero, A. Fede, D. Giubrone, L. D'Andrea

Chirurgia Ortopedica, Messina

Introduction: External fixation is a technique for shaft fracture's treatment used also in pediatric traumatology with good results; the external fixator allows a rapid surgery time, a good articular function and preserves the cartilage physeal plate.

Materials and Methods: We considered a group of 20 pediatric patients with humeral shaft fracture treated between 2004 to 2006; in all patients we used an Orthofix Axial External Fixator. A rehabilitation protocol during 30 days followed surgery.

Results: External fixator was maintained for a mean time of 60 days; we used the DASH score to value the results: all patients treated had a good satisfaction and recovered the function of upper limb. In one case we observed a refracture after external fixation removal initially treated with a cast and after with plate osteosintheys with plate and growth factors. In five cases we had a superficial pins infection treated with antibiotic therapy.

Conclusions: External fixation is a good method for treatment of humeral shaft fracture in pediatric age because is minimally invasive, respects the cartilage physeal plate and may usually a good compliance of little patients. 


\section{1}

\section{Dinamic Osteosynthesis in Humeral Shaft Fractures}

\author{
A. Fede, D. Pontoriero, N. Catena, L. D'Andrea, \\ C. Impallomeni \\ Chirurgia Ortopedica, Messina
}

Introduction: Axial external fixation is a rapid method of osteosynthesis for humeral fractures that may obtain a good reduction and recover articular function of shoulder and elbow. Marchetti Vicenzi nail is an intramedullary system for dynamic osteosynthesis used in humeral fractures with similar surgical timing and results, compared to external fixation. The authors evalued the results obtained with the two methods of dynamic osteosynthesis.

Materials and Methods: We considered a group of 46 patients ( 25 male and 21 female) between 2002 to 2005 with humeral shaft fractures treated in 15 cases with Marchetti Vicenzi Nail and 31 cases with Orthofix Axial External Fixator; the mean time of surgery was about 50 minutes. Fifteen days after surgery the patients began the rehabilitation with active and passive kinesis of shoulder and elbow.

Results: We made a clinic and radiographic evaluation at 1, 2, 3, 6 and 12 months after surgery and used the DASH score for evaluation. Twelve months after surgery 41 patients healed and returned to preinjury activity. No evidence of difference between Marchetti Vicenzi nail and external fixation about the time of healing. No evidence of major neurologic complications. We noticed non union in 4 cases treated with external fixation, and in 1 case treated with Marchetti Vicenzi nail; in these patients we removed the first imp.

\section{2 \\ Use of Transosseus Anchors in the Treatment of Biceps Tendons Rupture}

\author{
D. Pontoriero, N. Catena, A. Fede, L. D'Andrea, G. Risitano \\ Chirurgia Ortopedica, Messina
}

Introduction: Biceps tendons ruptures aren't a frequent event; the lesion of distal biceps tendon occurs more frequently than the proximal. The mechanism is usually traumatic in younger patients and degenerative in older. The authors describe their experience about the use of transosseus anchors in the treatment of biceps tendon ruptures.

Materials and Methods: We considered a group of 6 patients ( 5 distal tendon and 1 long head tendon rupture) treated from 2000 to 2006. The distal rupture had a surgical treatment with an anterior approach to radial tuberosity and suture with 2 transosseus anchors (Mitek in 3 cases, Arthex in 2 cases). For proximal lesions we use a single incision in correspondence of the muscle mass and after the tendon's finding we translate the same throught a proximal subcutis tunnel and using a second anterior incision we arrived to humeral head using a single transosseus anchor. After surgery we use an immobilization for 4 week and 12 weeks of rehabilitation.

Results: Medium follow up was of 4 years; we used the Ewald Score System for distal rupture and the Constant Murlay Score for proximal lesion. All patients had a total articular and fuctional restoring and all returned to preinjury activity levels by 16 weeks. No evidence of anchors mobilitation or heterotopic bone formation occurred; in one patient had a neuroapraxy of sensitive radial nerve, healed in 25 days.

Conclusions: Transosseus anchors are an excellent method of fixation for these lesions; the surgical technique are simply, rapid and allows an anatomical tendon's reconstruction with a mininal incidence of complications especially neurological.

\section{3 \\ Our Experience with Mipo Technique \\ T. Tavernini, A. Fede, D. Pontoriero, N. Catena, C. Impallomeni \\ Chirurgia Ortopedica, Messina}

Introduction: Inconveniences and problems related to conventional O.R.I.F. and I.N. in the treatment of distal femoral fractures are well established. These problems have been frequently associated with extensive exposure of the fracture site and sometime with the technical features of the devices themselves. MIPO is a technique that allows to avoid direct exposure of the fracture site, minimizing soft tissues trauma, preserving vascular perfusion leading to an early healing and a low rates of infections.

Materials and Methods: We performed 25 patients, from 2003 to 2006, 14 males and 11 females; our cases were classified according with $\mathrm{AO}$ classification for distal femoral fractures. MIPO technique was performed with LISS plating System.

Results: Follow up range went from at least 31 days to maximum 81 days. 24 of our patients underscored good or excellent results (in terms of fracture healing and r.o.m.) only in 1 case we observed implant mobilization.

Conclusions: Our short experience with this device is not enough to give a final assessment about this technique, but high percentage of our good results and the data collected from Literature seems to assign to LISS system a leading role in the treatment of distal femoral fractures.

\section{4}

Treatment of Osteochondral Lesions of the Talus with Autologous Stem Cells: Preliminary Experiences
G. Di Napoli, E. Grosso, M.F. Suozzi, V. lacono, D. Rosa
Department of Orthopaedic Surgery, University 'Federico II' - Naples

Introduction: Osteochondrosis dissecans of the talus is a pathology characterized by cartilaginous and subchondral bone detachments. The recent 'plasticity theory' suggest that adult stem cells can differentiate into a wide range of specialized cell types, therefore they could take part in the processes of repair of the osteochondral damage.

Methods: We have implanted autologous stem cells in two male patients of 19 and 23 years. Before the operation, the patients must make one RMN, to repeat to 3, 6, 12 months from the operation; and 
one biopsy after 18 months. We used adult bone marrow stem cells isolated from cristae iliacae $\left(6 \times 10^{6}\right.$ in the first patient and $7 \times 10^{6}$ in the second patient). Then stem cells are processed using MACS ${ }^{\circledR}$ immuno-magnetic technology to obtain high rate of CD $34+/ C D$ $133+$ purified stem cells. The osteochondral lesions was filled with blood clot containing stem cells previously processed. We used Chondro-gide collagenic membrane to cover the site of lesion, to prevent intrarticular stem cells dispersion. Microenvironment can drive stem cells to differenziate in chondrocytes.

Results: There are not reliable outcomes because the group that we have is too much small and the follow-up is short. At 3 and 6 months RMN shows that the repair tissue emits variable and heterogenous signals.

Discussion: These preliminary human experiences can be useful to understand the real resources of stem cells and also to intrigue the scientific community. The mean limits are the absence of ideal scaffolds and high cost of this technology.

\section{5}

\section{Total Knee Replacement in Obese Patient: Problematic of Treatment}

\author{
V. Bellotti, S. Guarino, A. Russo, G. Di Napoli, D. Rosa \\ Department of Orthopaedic Surgery, University 'Federico \\ II' - Naples
}

Introduction: Excessive body weight contributes to the development of osteoarthritis. Obese patients often need a joint replacement surgery that, due to co-morbidities of these patients, introduces to remarkable problematic of treatment.

Methods: From January 2000 to February 2006 we performed TKR for 47 obese patients, 18 men (BMI of $39.15 \pm 2.27 \mathrm{~kg} / \mathrm{m}^{2}$ ) and 29 women (BMI of $37.15 \pm 1.75 \mathrm{~kg} / \mathrm{m}^{2}$ ) with an average age of 62 years old. We compared them to a control group with normal BMI. During a medium follow-up of 24 months we evaluated the clinical results, the problematic of treatment before, during and after surgery, and late complications.

Results: Clinical outcome was lower in obese patients. Before surgery, co-morbidity with other pathologies caused more specialistic consults and instrumental exams; the anaesthesiological risk was higher. They needed a major surgical incision and soft-tissue separation; prosthetic alignment and ligamentous balance were more difficult. Infection rate was higher, due to the longer operating time, to surgical exposure, to lower blood supply in fat tissue and to a deficit in immune response. The rate of DVT was higher.

Conclusions: TKR is a validated surgical procedure when indicated. By the way, results are not so sure in obese patients. They have a higher rate of co-morbidity, so a multidisciplinary approach is favourable to improve and assess pre-operative clinical conditions.

\section{6}

\section{Mini-Invasive Approach for Knee Surgery}

\section{Iacono, E. Astarita, V. Bellotti, G. Balato, D. Rosa \\ Department of Orthopaedic Surgery, University 'Federico II' - Naples}

Introduction: Mini-invasive approach represents the new trend of the recent evolution in orthopedics surgery in all of its sectors. In the knee arthroplasty, mini invasive surgery, is sought both in the use different surgical approaches and in the use of redoubt dimensions prothesis.

Discussion: Different surgical approaches are turned to the reduction of the cutaneous incision, of muscles, tendons and ligaments dissection, of bony resection, and to minimal interruption of nervous and vascular structures. This approach leads to the reduction of haematic losses, less pain for patients, a faster recovery with a reduction of the hospitalization and a remarkable reduction of the costs. In the last years small dimensions prothesis were built. They remove a smaller part of bone, preserve the functional structure of it and allow easy removal of it in case of revision; in fact, they replace only the zone with the cartilaginous damage, proposing themselves as valid alternative to total articular arthroplastics.

Conclusions: Mini-invasive surgery is not only a reduction of the cutaneous cut. It is a smaller incision, appropriate exposition and the least structures dissection that gives the maximum satisfaction to patients with a remarkable containment of the costs both for the patients and the community.

\section{7}

Total Knee Arthroplasty in Patients with Severe Deformities Due to Rheumatoid Arthritis: Middle Term Results

\section{A. Silvestro, F. Aquino, L. Sannino, G. Di Napoli, G.L. Federico, D. Marinò \\ Department of Orthopaedic Surgery, University 'Federico II - Naples}

Introduction: In RA patients with a very aggressive disease, pathological changes in the knee occur in $90 \%$ of cases in the end-stage period. These changes are often accompanied by severe deformities, such as flexion contracture and valgus deformity. In order to provide soft tissue balance, these deformities require additional surgery before implantation of the prosthesis, such as posterior release and lateral soft-tissue release. Sometimes are necessary bone stocks.

Methods: We evaluated the middle term fixation of 36 cemented total knee prostheses in 31 patients with rheumatoid arthritis ( 27 female and 4 male). The mean age at operation was 48 years ( 31 to 69 ) and were examined at a mean follow-up of 7.4 years ( 4 to 11). Prosthetic review included clinical evaluation (KS score based on 100 points) and complete radiological evaluation enabling the postoperative position of the implants to be examined. Fundamental a precocious rehabilitation.

Results: There were no local postoperative complications. According to the KS score raised from 38 preoperatively to 88 . The 
results of 32 of the 36 patients were rated good or excellent; 3 were sufficient and 1 insufficient due to mechanical complications. Radiolucencies revealed in 15 per cent of cases.

Discussion: The implantation of cemented primary prosthesis seem very satisfactory in RA. Only one reoperation was required because fixation failure. The middle term success was of $97 \%$.

\section{8}

Compression Neuropathy of the Motor Fibers of the Median Nerve. Case Report and Rewiew of the Literature

\section{G. Rizzello, M.C. Meloni, N. Martinelli, F. Leonardi, R. Quadrini, V. Denaro \\ Orthopaedic Department, Campus Bio-Medico Hospital, University of 'Campus Bio-Medico' Rome}

Introduction: In CTS, selective electrophysiological involvement of median motor fibres is rare and varies from 0.6 to $1.2 \%$, depending the sensitivity of the test used. We found a case of compression neuropathy of the median nerve which clinically and electrophysiologically showed only motor signs and symptoms. Decompression of the median nerve produced excellent results.

Methods: A 58 year-old male patient presented with a 3-month history of pain and decreased strength in the dominant left thumb. The studies showed that there was a selective compression of the terminal median nerve motor branch to abductor pollicis brevis.

Results: During the surgical procedure, the exploration to the junction of the motor branch did not reveal any anatomical variation of the motor branch of the median nerve or any other structure compressing. Ten weeks after surgery the patient had no difficulty in using his left hand.

Discussion: Isolated motor branch involvement is described in literature. In our case, although the clinical and electrophysiological findings suggested a selective compression of the thenar branch, we did not find either selective compression of the thenar branch or anatomic courses variation of the median nerve and its branch in the carpal tunnel during the operation.

239

Autologous Bone Marrow Cells Concentrate with Platelet-Rich Fibrin on Corticocancellus Bone Allograft for Posterolateral Multilevel Cervical Fusion: Case Report and Technical Note

G. Vadalà', Alberto C. Di Martino', M. Andrea1, G. Rizzello', M.C. Tirindelli ${ }^{2}$, V. Denaro ${ }^{1}$

'Department of Orthopaedic and Trauma Surgery, Campus Bio-Medico University, Rome, Italy; ${ }^{2}$ Department of Haematology, Campus Bio-Medico University, Rome, Italy

Introduction: The outcomes of posterolateral multilevel spine fusion in difficult clinical settings such as old multi-diseased osteoporotic patient remain unpredictable. The osteoprogenitor cells in bone marrow decrease with aging without losing their osteogenic potential. Bone marrow cells (BMCs) from iliac crest aspiration can be concentrated in the operating room and the platelet-rich fibrin (PRF) can be obtained from peripheral blood as source of autologous ostoprogenitor cells and growth factors respectively. The objective of this report is to describe the use of autologous BMCs concentrate enriched with PRF on corticocancellous bone allograft to improve multilevel posterolateral cervical fusion in a case of a 88-year-old men affected by cervical stenosis and subjected to decompression and fusion.

Method: We presented the case of a posterolateral C3-C7 instrumentation with apposition of posterolateral corticocancellus bone allograft augmented with autologuos BMCs concentrate from iliac crest aspiration enriched with PRF from peripheral blood.

Results: At 6-months follow up the bone allograft shows consolidation signs evaluated by Lateral Dynamic X-Rays and CT scan.

Discussion: This paper describes a simple and effective method for potentially improving fusion rate in aged osteoporotic patients by using corticocancellus bone allograft augmented with autologus BMCs concentrate from iliac crest enriched with PRF from peripheral blood, rapidly obtained before the surgical procedure.

240

Intervertebral Disc Stem Cell Therapy: Mesenchymal Stem Cells Differentiation without Cell Fusion Mediated by the Interaction with Nucleus Pulposus Cells
G. Vadalà', F. Spiezia1, V. Denaro ${ }^{1}$
${ }^{1}$ Department of Orthopaedic and Trauma Surgery, Campus Bio-Medico University, Rome, Italy; ${ }^{2}$ Department of Orthopaedic Surgery, University of Pittsburgh, Pittsburgh PA, USA

Introduction: Introduction of exogenous cells to supplement and replenish intervertebral disc cell population offers a potential approach to treat intervertebral disc degeneration (IDD). Recent evidences showed that intradiscal injection of MSC effectively alter the course of IDD in vivo, and the regenerative potential may result from up-regulated extracellular matrix protein synthesis mediated by MSC and NPC interaction. The objective of this study is to investigate the mechanisms of the interaction between NPC and MSC.

Methods: Using a double labeling cell system and flow activated cell sorting, we quantitatively analyzed changes in the gene expression profile of human male MSC and female NPC after co-culture in a three-dimensional system that allows cell-matrix-cell interactions typical of the nucleus pulposus. Furthermore, we analyzed for cell fusion in the cell interaction by fluorescence in situ hybridization (FISH) for $\mathrm{X}$ and $\mathrm{Y}$ chromosomes, using a three-dimensional culture system to allow cell-to-cell interactions conducive to cell fusion.

Results: After 2 weeks of co-culture cell interaction in a cellmatrix-cell environment induces a change in MSCs towards a more chondrogenic gene expression profile indicating MSC differentiation, and NPC gene expression changes in matrix and chondrogenic genes demonstrating only a modest trophic effect of MSC upon NPC. 
Moreover, FISH analysis demonstrated that cell fusion is not responsible for MSC plasticity in the interaction with NPCs.

Discussion: This study clarifies the mechanism of MSCs and NPCs interaction, supporting the use of undifferentiated MSC for stem cell therapy for IDD treatment.

\section{1}

\section{Pathology of Femoral Heads Following Vascularized Fibular Graft}

\author{
M.C. Meloni, V.L. Fornasier, G. Rizzello, V. Denaro \\ ${ }^{1}$ Bone and Joint laboratory, Division of Pathology, \\ St. Michael's Hospital, University of Toronto; \\ ${ }^{2}$ Orthopaedic Department, Campus Bio-Medico Hospital, \\ University of 'Campus Bio-Medico' Rome
}

Introduction: Free vascularized fibular graft for osteonecrosis of the femoral head is a well established procedure based on the assumption that the graft will provide mechanical support, blood supply to the osteonecrotic head and to introduce mesenchymal stem cells into the affected area of the femoral head.

Methods: We reviewed 27 cases to delineate the pathological features of femoral heads with AVN treated by vascularised fibular grafts which were retrieved at revision surgery.

Results: The length of the graft was divided into three zones: zone 3 the femoral neck; zone 2 the lower femoral head or 'metaphysis'; zone 1 the more apical or epiphyseal component of the femoral head. The intention was to follow the vascularization of the pedicle and the changes in the three diverse areas of the specimen. The graft showed incorporation with the host bone. In the pedicle there was preservation of vascular patency and tissue viability.

Discussion: However, this healing process involved a slow reparative resorptive activity which undermined the joint surface. This could suggest that non-resorbable materials in place of tissue grafts can be expected to avoid the negative effect of creeping substitution as an undermining force in the repair and revascularisation of the necrotic area in the head.

\section{2}

\section{The Biplanar Austin and Triplanar Boc Osteotomies for Mild Hallux Valgus Correction: Comparison of Pre- and Post-Surgical Characteristics using Plantar Pressures}

\section{F. Cancilleri, A. Marinozzi, N. Martinelli, M. Ippolito,} F. Spiezia, M.C. Meloni, V. Denaro

Orthopaedic Department, Campus Bio-Medico Hospital University of 'Campus Bio-Medico' Rome

Introduction: A retrospective analysis was performed to compare the effects of Austin and Boc osteotomies on plantar pressure distribution and their relevance to the clinical outcome.
Methods: This study evaluated 60 patients who underwent operations for mild to moderate hallux valgus deformities over a period of 2 years. 30 Austin and 30 Boc osteotomies were performed on 30 patients with a mean follow-up of 37 months (29-56 months). The plantar pressures were documented with the Diagnostic Support system ${ }^{\circledR}$ footplate.

Results: The average postoperative American Orthopaedic Foot and Ankle Society (AOFAS) scores after Austin and Boc osteotomies were 81.9 and 86.4 , respectively. The pressure distributions under the fourth and fifth metatarsal head were similar in both study groups $(p>0.05)$. After Austin osteotomies, decreased load bearing was noted under the hallux $(\mathrm{p}=0.01)$, coupled with a persistent overloading of the second and third metatarsal heads $(\mathrm{p}>0.05)$. But after the Boc procedures, decreased weight bearing under the hallux was coupled with better load distribution beneath the second and third metatarsal heads $(\mathrm{p}<0.05)$. Correlation of AOFAS score and pressure variables demonstrated a significant negative correlation with hallux and central metatarsal heads loading $(\mathrm{p}<0.01)$.

Discussion: This study shows that adequate forefoot loading is imperative for a better outcome of the procedure. Our results showed how distal triplanar Boc osteotomy of the first metatarsal head, compared with the biplanar Austin osteotomy, determines a better load distribution on the metatarsal heads and restabilish a better biomechanically propulsive gait phase.

\section{3 \\ Off-Label use of Mesna in Dupuytren's Disease}

\section{A.D. Martino, V. Costa, N. Martinelli, M.G. Zagami, V. Denaro}

Orthopaedic Department, Campus Bio-Medico Hospital, University of 'Campus Bio-Medico' Rome.

Introduction: Dupuytren's disease is a benign fibroproliferative disorder, associated with progressive retraction of the palmar fascia that leads to digital flexion deformities, especially for the fourth and fifth finger. MESNA (sodium-2-mercaptoethanesulfonate2), with a molecular formula of $\mathrm{C}_{2} \mathrm{H}_{5} \mathrm{NaO}_{3} \mathrm{~S}_{2}$, thanks to his structure, produces mucolysis by disrupting disulfide bonds of the mucus polypeptide chains. MESNA appears a candidate for applications in Dupuytren's disease: the action on disulfide bonds could be important because the high concentration of sulfide GAG such as Dermatan sulfate and Choindroitin sulfate in the palmar fascia of patient affected by Dupuytren's disease.

Methods: We have histologically analyzed a fibrotic cord, extracted intraoperatorially from a Dupuytren's palmar fascia, dipped into MESNA for 12 hours (at the concentration of $400 \mathrm{mg} / 4 \mathrm{ml}$ ) or in fisiologic solution as control group, to verify the possible role of MESNA in the treatment of Dupuytren's disease.

Results: The samples analyzed have showed some differences because the tissue dipped into MESNA showed a collagen fibers separation not present in the control group dipped into fisiologic solution.

Discussion: Such confirmation, together with other investigations in literature, could hypothize local application of MESNA: during surgery in the fasciectomy of patients affected by Dupuytren's contracture thanks to the break of disulfide bonds connecting matrix 
GAG molecules. The extracellular matrix disorganization could be very useful because the palmar fascia's pathological compactness/ stiffness exposes the patient to frequent complications like skin necrosis and partial removal of pathological tissue.

\section{4}

Lengthening of the Forefoot Bones in Children

L.P. Spreafico, A. Kirienko

Chirurgia Ortopedica, Lecco

Introduction: This study investigated MT and phalanx lengthening by distraction lenghthening for congenital or post-traumatic shortening in $23 \mathrm{MT}$ and 3 phalanx of the hallux, in 17 patients.

Methods: 7 males and 10 female, mean age 13 yrs ( $8-17$ yrs). 2 patients with Turner syndrome, brachymetatarsia of 4th MT in 9 ( 3 bilateral) and post-traumatic and congenital defect of 1 st metatarsal in 3.3 patients had stump of the proximal phalanx of the hallux. We performed lengthening using semicircular and Ilizarov's mini-fixator, fixing bones to the ex-fix by crossed K-wires, performing percutaneous osteotomy and then distraction five days after surgery. Early weight bearing was always allowed. The rate of lengthening was of $0.75 \mathrm{~mm}$ per day, slowed to $0.5 \mathrm{~mm}$ after 2 weeks.

Results: The average in length was $17.3 \mathrm{~mm}(12-25 \mathrm{~mm})$, equivalent increase of $36 \%(25-46 \%)$ and a mean healing index of 61 days $/ \mathrm{cm}$. AOFAS average score was 85.3 (range 53-100). The most common complication was stiffness of MT-Ph joint (6), subluxation of this joint (2), angular deviation (5), pin track infection (4), 1 retard of consolidation. We did not need to use bone grafting and no $\mathrm{N}-\mathrm{V}$ complications were encountered.

Conclusion: Gradual distraction gives a fair precision lengthening for forming the physiological arch of metatarsals heads. It also permits a contemporarily correction of deformities without bone grafting and soft tissue modifications.

\section{Pediatric Surgery}

\section{5 \\ Perinatal Risks Factors for Surgical Necrotizing Enterocolitis}

\section{M.G. Di Napoli, V. Rossi, F. Macchini, D. Nicolò \\ Scuola di specializzazione in Chirurgia Pediatrica, Milano}

Introduction: We observed a reduced incidence of necrotizing enterocolitis (NEC) despite increased survival of extremely premature infants over the last two decades. A different susceptibility pattern to NEC according to gestation has been proposed. We aim to examine the influence of perinatal risks factors for NEC in infants referred to our hospital for surgical NEC.
Methods: Surgical NEC incidence, perinatal risks factors and neonatal outcome were examined in a cohort of 44 infants from our Neonatal Intensive Care Unit admitted during 1996-2006 for proven NEC (Bell stage II and above).

Results: A total of 21 (48\%) infants developed NEC requiring surgical therapy. $90 \%$ were premature infants, with gestational age between $25^{+5}$ and 31 weeks. $66 \%$ were very low birth weight, $38 \%$ were small for gestational age (SGA) and $47 \%$ extremely low birth weight; the birth weight mean was 1,524 g. 67\% had Hyaline Membrane disease; $55 \%$ had patent ductus arteriosus needing treatment. Radiographically $80 \%$ of affected infants display sign of pneumoperitoneum, no one showed portal vein gas or fixed distended bowel loop. The mortality rate was $29 \%$, surgical complications were reported in $62 \%$ ( $24 \%$ stricture formation).

Discussion: Lower gestational age was associated with higher incidence of surgery for NEC; the post-operative mortality was comparable between lower and higher gestation infants. Prematurity is still the only proven risk factor for NEC.

\section{6 \\ Decreased Incidence of Necrotizing Enterocolitis (NEC) in Maternal Milk-Fed Preterm Infants}

M.G. Di Napoli, V. Rossi, F. Macchini

Scuola di specializzazione in Chirurgia Pediatrica, Milano

Introduction: Necrotizing enterocolitis (NEC) is a devasting intestinal disease of premature infants. Maternal milk has been suggested to be partially protective against NEC. Many studies suggest the anti-inflammatory effects of specific factors found in human maternal milk on human fetal intestinal epithelial cells.

Methods: We analyzed the feeding regimen (breast or formula milk, timing, initial volume, advancement) of 21 infants who developed proven NEC (Bell stage II and above), requiring surgical therapy, admitted during 1996-2006 in our surgical Unit (group 1). We collected the same data in a cohort of 21 infants (with similar gestational age, birth weight and perinatal risks factors) admitted during the same period in our Neonatal Intensive Care Unit (group 2).

Results: In the group 1, $20(95 \%)$ infants were artificially fed with formula milk: 6 (29\%) received only artificial milk; 7 (33\%) received both formula feeding and freezing collected human milk; 3 had total parenteral nutrition; 2 received intravenous (IV) glucose solution; 1 had IV C-formula; 1 had IV F-Formula; 1 was breast fed. In the group 2, 19 (90\%) infants were breast fed and 2 received both maternal and artificial milk; only 1 showed signs of NEC (Bell stage I, treated and resolved with medical therapy), 1 had surgical treatment for an isolated intestinal perforation.

Discussion: These results suggest the protective effect of maternal milk against NEC. 
247

\section{Chronic Food Refusal after Long-Term Nasogastric Tube Feeding in Child with Liver Transplantation.}

M.G. Di Napoli, V. Rossi, F. Macchini

Scuola di specializzazione in Chirurgia Pediatrica, Milano

Introduction: Enteral feedings delivered by nasogastric tube are often used in infants to ensure adequate caloric and nutrient intake. But there is a critical period involved with oral feeding during the first year of life; children who were in transition from tube to oral feeding in this period may display oral-motor, sensory and developmental feeding problems.

Methods: We evaluated the changes in feeding behaviour and the developing of poor feeding skills of a 8 month-old baby who underwent liver transplantation for biliary atresia.

Results: Before the prolonged and frequent hospitalizations the baby feeding behaviour was normal. After she showed: first a chronic food refusal, then swallowing difficulties. She used to leave the food in the oral cavity without chewing or swallowing it. She had no signs of organic disease or anorexia nervosa. She received occupational therapy intervention (the team included gastroenterologist, nutritionist, behavioral psychologist, occupational and speech therapist) and after two years of feeding induction program, continued also at home by the trained parents, she began to eat normally.

Discussion: Nasogastric tube feeding is frequently necessary, especially in children who underwent gastrointestinal surgery, to overcome the inadequate caloric intake during a long-term hospitalization but the need for exclusive tube feeding should be minimized. To avoid iatrogenic feeding problems initial attempts to achieve nutritional goals in malnourished children should be via the oral route.

\section{8 \\ BOIX-OCHOA Fundoplication for Treatment of Gastroesophageal Reflux in Children}

\section{Rossi, M.G. Di Napoli, S. Luzzani \\ Scuola di Specializzazione in Chirurgia Pediatrica, Università di Milano}

Introduction: 12 children with gatroesophageal reflux underwent BOIX-OCHOA plasty. Indication for surgery included: erosive esophagitis, drug therapy nonresponder, respiratory distress associated to gear, peptic esophageal stenosis, short esophagus as result of esophageal atresia.

Methods: A severe neurological compromission was present in 3 patients. Diagnostic evaluation included: $24 \mathrm{~h}$. ph-monitoring, radiological study, endoscopy, gastric emptying (by us).

Results: Clinical and instrumental follow-up, from 6 months up to 4 years, demonstrated satisfactory results in all cases, except for one neurologically disabled patient.

Discussion: No side effects such as gas-bloat and retching syndrome, often observed after nissen fundoplication, were noted.
249

\section{Is Ultrasonography Contribute Decision- Making in the Assessment of Children with Suspected Appendicitis?}

V. Rossi, M.G. Di Napoli, R. Arnoldi

Scuola di Specializzazione in Chirurgia Pediatrica, Università degli studi di Milano

Introduction: A study was performed to asses the impact of ultrasound on decision making in pediatric appendicitis.

Methods: A retrospective case review was conducted of patients undergoing appendectomy during the six year period, January 1998 to December 2004. Presenting signs and symptoms, sonographic findings and histopathological results were recorded. 237 children (154 M, $83 \mathrm{~F}$ ) were studied. The age range was 2 to 17 years (mean 11). Ultrasonography was performed on 25 patients. Of these, 9 had perforated appendix -5 localized and 4 generalized peritonitis, 12 acute appendicitis and 4 normal appendicitis.

Results: The overall diagnostic accuracy was $88 \%$, and sensitivity $95.2 \%$. US prevented surgical delay in $12 \%$ of the patients.

Discussion: We conclude that US is a realiable tool for decisionmaking in pediatric appendicitis.

250

\section{Laparoscopy for Non-Palpable Testis}

V. Rossi, M.G. Di Napoli, S. Fedeli, A.C. Agliardi

Scuola di Specializzazione in Chirurgia Pediatrica, Università degli Studi di Milano

Introduction: 22 patients in 3 years period were diagnosed as: primary non palpable testis (group A-11); piping testis (group B-3) and post groin exploration (group C-8).

Methods: The average age at laparoscopy was: groups $1.83 \pm 0.4$ years and group C $8.38 \pm 2.75$ years. Imaging studies to locate testicular tissue pre-laparoscopy include only abdominal US. Exploratory laparoscopy was performed in all 22 patients.

Results: Testicular elements adjacent to the internal were found in 6 patients (group A-3, group B-3). In two patients (groupA-1 and group C-1), testes was found along the gutter of the affected side. Laparoscopic orchidopexy was performed in 9 patients of them, 2 patients had high ligation of testicular vessels. Testicular tissue was not found in 16 patients (group A-9, group C-7). Groin exploration in all these patients, show no testicular tissue macroscopically.

Conclusion: Laparoscopy is an effective diagnostic and operative modality for non-palpable or piping testis. 


\section{1}

\section{Oxygen Riduction of the Intussusception in Children}

V. Rossi, M.G. Di Napoli, S. Bashkcon, D. Nicolò, C.A. Dell'Agnola

Scuola di Specializzazione in Chirurgia Pediatrica, Università di Milano

Introduction: The management of 84 cases of intussusception is referred.

Methods: Age ranged from 15 days to 5 years. Diagnosis was made in 82 cases by plain X-ray and barium enema. In 2 cases of bowel perforation the diagnosis was made at surgery after a plain $\mathrm{X}$-ray only. Hydrostatic reduction was attempted preoperatively in 82 cases and it was successful in 11 cases (13\%), 3 by ultrasound (US) guide, 8 by traditional barium enema.

Results: 73 children underwent surgery. In 7 cases $(8.3 \%)$ intestinal resection and direct anastomosis were necessary (in 2 of them an ileo-ileal intussusception was found at surgery). In all cases an ileo-cecal pexis was performed to prevent the post-operative relapsing of intussusception.

Discussion: Our data indicate a high rate of surgical treatment. We did not observe complications and not recurrence probably because we performed ileo-ceco-pexis. No one died.

\section{2 \\ Semen Quality and Gonadotropin Levels in Patients Operated upon for Cryptorchidism}

V. Rossi, M.G. Di Napoli, D. Nicolò, A.C. Agliardi

Scuola di Specializzazione in Chirurgia Pediatrica,

Università di Milano

Introduction: In a long term follow-up study we compared preoperative testicular position, age at orchiopexy and morphology of testicular biopsies, investigated at orchiopexy to sperm analysis results, testicular volume, and serum follicle-stimulating hormone and luteinizing hormone levels at follow-up in 46 men with a history of surgical correction of cryptorchidism in childhood.

Methods and Results: They had undergone orchiopexy at age 1.5-12.0 years, 24 for unilateral and 22 for bilateral cryptorchidsm. $11(46 \%)$ of 24 patients with unilateral and $7(32 \%)$ of the 22 with bilateral undescended testes had a normal sperm analysis, whereas in a men with impaired spermiogenesis oligo-asthenozoospermia was the predominant feature. $3(13.6 \%)$ patients with bilateral cryptorchidism showed azoospermia, but none of patients with unilateral cryptorchidism did.

Discussion: Neither the age at orchiopexy nor the average germ-cell count per cross-sectioned seminiferous tubule of testicular biopsies examined at orchiopexy correlated significantly with subsequent sperm analysis results or gonadotropin levels at follow-up. The pre-treatment testicular position has a high prognostic value for prediction of subsequent sperm analysis results and serum gonadotropin levels.
253

\section{Treatment of Cryptorchidism with LHRH} Nasal Spray

\author{
V. Rossi, M.G. Di Napoli, R. Arnoldi, A.C. Agliardi \\ Scuola di Specializzazione in Chirurgia Pediatrica, \\ Policlinico IRCCS Milano
}

Introduction: 53 prepubertal boys with untreated cryptorchidism were investigated in this study. 38 boys had unilateral and 15 boys had bilateral cryptorchidism.

Methods: $200 \mu \mathrm{g}$ syntetic LHRH in a $0.2 \%$ aqueos solution (Kryptocur) were sprayed 3 times daily into each nostril (total dosage $1.2 \mathrm{mg} / \mathrm{d}$ ). The duration of therapy was 4 weeks. The boys were examined by palpation before and then 2,4 weeks and 6 months after commencement of treatment.

Results: Descent was achieved in $67.6 \%$ of testes; in the boys with unilateral cryptorchidism descent was achieved in $57.9 \%$ and in the boys with bilateral cryptorchidism in $80.0 \%$. Follow-up control 6 months later showed relapse in 14 cases $(30.4 \%)$.

Discussion: Treatment of cryptorchidism by intranasal application of LHRH, 3 times $200 \mu \mathrm{g} / \mathrm{d}$ is very effective. Administration is simple, painless and well tolerated. Follow-up is recomanded, however, in view of the relatively high relapse rate.

\section{4 \\ Triple Incision to Treat Phimosis in Children: An Alternative to Circumcision}

V. Rossi, M.G. Di Napoli, R. Arnoldi, A.C. Agliardi

Scuola di Specializzazione in Chirurgia Pediatrica, Università degli studi di Milano

Introduction: Circumcision is the accepted operation to treat phimosis. However, when the purpose is to achieve retractility of a narrow foreskin to avoid further scarring and phimotic development after recurrent balanitis, a preputial plasty might be sufficient. Several methods with single or multiple incision have been introduced throughout the years. None of them seems to have gained general acceptance. Single plasties tend to give cosmetically unsatisfactory results. Still circumcision seems to remain the standard procedure for preputial relief.

Methods: A simple technique, where three longitudinal incisions are transverely sutured is described. It has been used in a series of 55 patients with good results, and seems to offer a good compromise between simplicity and cosmetical demands.

Conclusion: Triple incision is a simple, fast and safe technique for preputial relief and well accepted by the patients. 
Plastic Surgery

\section{5}

\section{The Vascular Architecture of Perforator Flaps: Haemodynamic Consequences}

\author{
A. Bulla1 , V. Coscia'², A.M. Cavazzuti ${ }^{3}$, F. Farace ${ }^{1}$, P. Mulas ${ }^{1}$, \\ C. Rubino' \\ ${ }^{1}$ University of Sassari, Department of Plastic \\ Reconstructive Surgery (Head: Prof. G.V. Campus), School \\ of Specialization in Plastic Reconstructive and Aesthetic \\ Surgery (Head: Prof. C. Rubino), ${ }^{2}$ Department of \\ Mathematics, School of Engineering, University of \\ Ferrara, Ferrara, Italy; ${ }^{3}$ Echographic Unit, University \\ Polyclinic, University of Sassari, Sassari, Italy
}

Introduction: Perforator flaps represent an important tool for the reconstructive surgeon. As a consequence of their harvesting, they are perfused and drained by vessels whose branches have been sealed, resulting in conduits with resistances in series, rather than in parallel, as in the normal systemic circulation.

Methods: In a series of ten patients, undergoing reconstructive surgery with perforator flaps, Echo-color-Doppler measurement of diameters, flow velocity and calculations of flow rate were made at the level of flap pedicle artery and skin perforator artery, pre- and post-operatively.

Results: In the donor area pre-operatively, blood velocity in skin artery perforator is lower than in the corresponding pedicle artery, whereas postoperatively, in perforator flaps, blood velocity in the perforator is higher than in the pedicle. Furthermore, the proportion of flow entering perforator artery and, hence, flap skin, is significantly higher than flow entering the same perforator artery before flap elevation.

Discussion: High blood velocity in perforator vessels may be favorable, as it is likely that it keeps relatively high in microcirculation of flap skin, preventing distal thrombi formation after revascularization. Blood supply to skin in perforator flaps is increased, so they may replace muscle flaps where the use of muscle flaps is indicated just because of their abundant blood supply. Axial flaps in all variants are the finest application of a thorough knowledge of vascular anatomy, perforator flaps may represent an exploitation of physiology of circulation in addition to anatomical knowledge.

\section{6}

\section{V.A.C. Therapy for Soft Tissue Defects: 7 Years Clinical Experience}

\section{Fraccalvieri, S. Carlucci, P. Bogetti, G. Datta, G. Verna, R. Pedrale, M.A. Bocchiotti, S. Bruschi}

Department of Plastic and Reconstructive Surgery University of Turin, Director: Prof. G. Bocchiotti

Introduction: V.A.C. (vacuum assisted closure) is a sore, ulcer and wound treatment technique.
Methods: 181 patients ( 60 females, 121 males) aged 28 to 89 were treated with V.A.C. from March 2000 to July 2007. 73 patients (59: vascular ulcer, 14: diabetic foot) were previously subjected to traditional and/or advanced treatment for 30 days to 10 years to prepare the lesion for skin grafting. In 95 patients (23: surgical wounds dehiscence; 31: pressure sores; 33: post-traumatic wounds; 8: radiodermatitis) the local condition (external fixer presence, lesion size, vascular tree, peri-lesion tissue damage) and/or general condition didn't allow surgical approach. 13 patients with pedicled and microsurgical partially congested flaps were treated with V.A.C. together with surgical revision. 25 patients were treated on lesions with over48-hour bone exposure, in 2 cases with signs of osteomyelitis.

Results: Local conditions significantly improved in 159 patients: for some the improvement allowed second intention recovery, in most of cases the lesion was prepared for skin grafting. In 24 cases V.A.C. was a life-saving aid decisively helping resolve serious infectious processes.

Discussion: V.A.C. is proving considerably versatile making it possible or easier to resolve a range of clinical situations. V.A.C. is to be used carefully in severe pain or osteomyelitis. If response to treatment after 2-3 medications is nil or minimal, it's necessary to consider a change in kind and/or in values of negative pressure exerted and/or a change in kind of materials used to medicate and some more parameters related to patient's posture and general condition before interrupting the therapy.

\section{7 \\ Use of Fibrin Glue in Body Contouring Following Massive Weight Loss: Our Experience}

M. Verga, S. Carlucci, S. Pau, E. Zingarelli,

M.A. Bocchiotti S. Bruschi

Department of Plastic and Reconstructive Surgery University of Turin, Director: Prof. G. Bocchiotti

Introduction: The ex-obese patient introduces peculiar problems related to metabolism, coagulation and tissue healing, with an increase of complications such as haematoma and sieroma that can be seen during the iter of body contouring. It results therefore important the continuous search of surgical techniques and medical devices that can reduce the frequency of these complications.

Methods: From 2004 to 2005 we have operated 30 ex-obese patients (previously treated with vertical gastroplasty) with abdominoplasty. The patients were randomized in two groups that differed for the employment of fibrin glue (TISSUCOL Baxter) through spraying as a haemostatic and adhesive device. We have analyzed in perspective way the results obtained in the two groups seeking some parameters that could objectively characterize the post-operative period.

Results: We have noted the possible onset of complications such hematoma and/or sieroma and considered as meaningful indexes the quantity in surgical drains related to the amount of removed tissue ( 170.8 vs. $246.8 \mathrm{ml} / \mathrm{kg}$ ), the mean hospitalization (6.6 vs. 7.2 days), the hospitalization related to removed tissue amount (4.37 vs. 4.6 days $/ \mathrm{kg}$ ), the mean loss of hemoglobin in the post-operative period $(2.48$ vs. $3.6 \mathrm{~g} / \mathrm{dl})$ and the mean loss of hemoglobin related to removed tissue (1.64 vs. $2.3 \mathrm{~g} / \mathrm{dl}$ per $\mathrm{kg}$ ). 
Discussion: The use of fibrin glue (TISSUCOL Baxter) determines in the post-operative period after abdominoplasty an improvement of some meaningful and objective parameters; this is ascribable first to the action on haemostatic and adhesive processes and then to the improvement of tissue cicatrization interface.

\section{8 \\ Ear Reconstruction with Engineered Cartilage. First Experimental Data}

\author{
S. Carlucci, M. Verga, S. Pau, E. Zingarelli, G. Datta \\ Department of Plastic and Reconstructive Surgery - \\ University of Turin, Director: Prof. G. Bocchiotti
}

Introduction: Currently for ear reconstruction we use an ear shaped skeleton of costal cartilage inserted in a pocket of skin expanded in mastoid region. The problems of this procedure are related to duration of operating times, to variability of surgeons' artistic skills, and to harvesting invasivity in the thoracic wall. In this work we try to define a reconstructive iter based not on costal cartilage, but on in-vitro cultivated one.

Methods: We have used human femoral chondrocytes and chicken sternal chondrocytes, cultivating them on non-woven fibre scaffolds of esterified hyaluronic acid in normal and sulfated form. The chondrocyte proliferation has been evaluated with spettrophotometry and the produced cartilage with histochemical analysis and immuno-histochemical analysis for type II collagene.

Results: We have identified in solfated esterified hyaluronic acid the ideal material for the chondrocytes growth, and we have produced both with chicken cells and with human ones, a cartilage of good quality, with abundant extracellular matrix.

Discussion: The engineered cartilage is of good histological quality but it still misses of solidity and it doesn't have a specific shape. Basing upon TAC data of the healthy ear, the virtual image of the ear to be reconstructed can be obtained, and therefore a solid model through fast prototipation can be created. Thanks to this model a biocompatible endoskeleton containing the hyaluronan scaffold could be built in which chondrocytes harvested from the healthy ear can be grown up with the purpose to get a preformed structure to be inserted under patient skin without costal harvesting.

\section{9}

\section{Surgical Anatomy of the Retroauricular Region}

\section{S. Carlucci, G. Datta}

Department of Plastic and Reconstructive Surgery, University of Turin, Director: Prof. G. Bocchiotti

Introduction: Both aesthetic and reconstructive ear surgery require a precise knowledge of the retroauricular region anatomy. In particular in ear reconstruction you have to preserve as much as possible the fascial layers useful to protect the cartilage grafts.
Methods: With cadaver dissection we precisely identified the anatomical structures of the mastoid, retroauricular and temporal regions, trying to mobilize them in several ways.

Results: Beneath the skin and the subcutaneous tissue a thick fascial layer is visible, which, in its cranial part, is called superficial temporal fascia and is elastic and not very fibrous; in its caudal part, this structure is called superficial mastoid fascia and contains within it the posterior auricular muscle and branches of posterior auricular artery. This fascia is thick, strong, fibrous and is a tissue able to provide protection and blood supply. Beneath the superficial mastoid fascia there is a thinner fibrous layer, the deep mastoid fascia, which is attached to periosteum of temporal bone. This fascia is cranially connected to a fibrous structure called innominate fascia. The latter separates the superficial temporal fascia from the deep temporal fascia, which directly covers the temporal muscle.

Discussion: In our opinion the perfect knowledge of the regional anatomy permits to have the utmost care for the tissues; in particular we have seen that acquiring familiarity in retroauricular region fascias management allows to preserve their integrity during the operations, permitting their use even in possible subsequent operations, in case of complications such cartilage exposure.

\section{0 \\ Increase of Postoperative Complications in Post-Bariatric Surgery: Analysis of Causes and Possible Solutions}

\author{
S. Carlucci, M. Verga, E. Zingarelli, S. Pau \\ Department of Plastic and Reconstructive Surgery, \\ University of Turin, Director: Prof. G. Bocchiotti
}

Introduction: The patients undergone to bariatric surgery (pbp) are subject to an increased risk of post operative complications, mostly following body contouring operations.

Methods: We have analyzed a population of pbp patients, undergone to plastic surgery operations (body contouring) and we have analized the occurred complications, the causes and the possible preventive solutions. The operations considered were abdominoplasty, reducing mastoplasty and mastopexy, thigh lift, brachioplasy.

Results: The major complications occurred were partial necrosis of the flaps, infections, hematoma and seroma, deep vein thrombosis, pulmonary embolism. Peculiar precautions have been taken and analized (convenient and continued antibiotic prophylaxis in the post operative period for at least 7 days, use of LMWH associated to antithrombus elastic socks or also with pneumatic intermittent compression, careful intraoperative mobilization of the big adipocutaneous flaps and convenient humidification of the same, careful observation in postoperative period of eventual bleedings).

Discussion: Pbp patients have shown an increased risk of complications. Possible causes seems to be an increased susceptibility to infections, important sizes of the mobilized flaps with an important bloody surface, increased risk of bleeding and thrombosis. The high incidence of complications during postoperative period in pbp patients imposes the use of peculiar and careful precautions in plastic surgery operations for body-contouring. Above mentioned precautions associated to a careful preoperative valuation of the patient, seems to be efficacious instruments for the control of the complication incidence. 
261

\section{Varioscope M-5: A New Type of Magnification System in Perforator Free- Flaps Surgery}

S. Chiummariello, C. Monarca, A. Domatsoglou, M. lera, C. Alfano, N. Scuderi

Università degli Studi di Perugia, Cattedra di Chirurgia Plastica e Ricostruttiva (Direttore: Prof. C. Alfano), Università degli Studi di Roma, Cattedra di Chirurgia Plastica e Ricostruttiva (Direttore: Prof. N. Scuderi)

Introduction: Perforator flaps allow the tissue transfer in a reliable approach with minimum donor site morbidity. Dissection of the small perforating vessels through the muscle/septum can be challenging; this places greater technical demands on the surgeon. Microsurgical success is closely linked on a clear and well-magnified field of vision. Our study is focusing on a new visual system Varioscope ${ }^{\circledR}$ M5 in the reconstructive procedures by perforator free flaps.

Methods: Varioscope ${ }^{\circledR}$ M5 is a new sophisticated high-end head-mounted vision system characterized by autofocus, zoom and pa-rallax control, powerful illumination and high-end documentation. The study was performed between 2004 and 2006 on 9 patients oncological disease affected. Varioscope ${ }^{\circledR}$ M5 has been employed in 9 anterolateral thight perforator flap, in head and neck surgery.

Results: Patients not highlighted failure at 20 days follow-up. 1 of 9 free flaps required re-exploration because of venous thrombosis. 1 flaps suffered a skin paddle ( $<20 \%$ loss) but there were no total flap loss.

Discussion: Varioscope ${ }^{\circledR}$ M5 can be a successful means of magnification for perforator flap transfers. It offers many advantages during the microsurgery. The surgeon is allowed closer access to the field as well as a wider view of the field for improved orientation. Its use is advocated on the grounds of cost-effectiveness, portability, efficiency and operator freedom. The ability to obtain swift magnification during different stages of an operation is an advantage particularly when dissecting perforator flaps and Varioscope's highest magnification is suitable, especially at the initial dissection of the perforator vessels.

\section{2}

\section{Fast Harvest of the Anterolateral Thigh Flap}

\section{E. Cigna', G.M. Feng' ${ }^{2}$, H.C. Chen ${ }^{2}$}

${ }^{1}$ University of Rome - La Sapienza, Department of Plastic Surgery, Italy; ${ }^{2}$ E-Da Hospital, I-Shou University, Department of Reconstructive Plastic Surgery Taiwan

Introduction: The anterolateral thigh flap was first reported by Song et al. in 1984, but it only recently become popular. The reason for the delayed popularity has been related to the learning curve of the perforator dissection.

Methods: The skin of the anterolateral thigh flap is supplied by septocutaneous or musculocutaneous perforators of the descending or transverse branch of the lateral circumflex femoral arterial system.
Results: The flap has been used for head and neck, extremity, and trunk defect microsurgical reconstructions. The large skin territory, reliability, and versatility of this flap have led to its popularity in soft-tissue defect reconstructions. When elevated, the anterolateral thigh myocutaneous flap usually includes a segment of the vastus lateralis muscle. However, the vastus lateralis is the largest compartment of the quadriceps femoris muscle and a partial sacrifice does not significantly impair the knee power or stability.

Discussion: We report the surgical technique of elevation of the flap and the thinning and ultra-thinning technique used in Taiwan for more than 3,000 flaps, which allows the harvest to be completed within 30 minutes.

\section{3}

\section{The Spontaneous Return of Sensibility in Non-Reinnervated DIEP Flap Breast Reconstruction: A Computerized Analyses}

D. Masia, G. Paolini, B. Longo, M. Angelini, F. Santanelli

\section{Azienda Ospedaliera Sant'andrea Roma}

Introduction: Breast reconstruction with DIEP flap is considered by most surgeons as the technique of choice for the more natural and permanent result than implant-reconstructed breasts. Recent studies advocate the superiority of reinnervated versus non-reinnervated free flap, but sensibility was measured with non accurate and subjective devices. Aim of our study was to evaluate with an objective and precise quantitative measurement method, the return of sensation in breasts reconstructed with DIEP flap, without reinnervation.

Material and Methods: 30 female that had a breast reconstruction by cutaneous-adipose DIEP flap were available for this study. The test was performed by the Dellon Test, at 6 and a 12 months postoperatively. Static and dynamic one and two points discrimination pressure thresholds were measured in $\mathrm{gm} / \mathrm{mm}^{2}$ in the four breast quadrants.

Results: Preoperative mean pressure thresholds for 1 and 2 PS in the breast were $16.0 \mathrm{gm} / \mathrm{mm}^{2}$ and $12.80 \mathrm{gm} / \mathrm{mm}^{2}$, while the DIEP flap showed $64.8 \mathrm{gm} / \mathrm{mm}^{2}$ and $61.0 \mathrm{gm} / \mathrm{mm}^{2}$ at 6 months post-op, and $41.9 \mathrm{gm} / \mathrm{mm}^{2}$ and $40.0 \mathrm{gm} / \mathrm{mm}^{2}$ at 12 months. Greatest recuperation at 12 months was found at the inferior-lateral part of the flaps.

Conclusions: This study first gives a quantitative accurate measurement of breast sensibility after reconstruction with nonreinnervated DIEP flap. DIEP flaps sensibility at 12 months was significant lower that non-operated breasts, but significant higher that DIEP flaps sensibility at 6 months, showing a recuperation. Static 1-point pressure thresholds, in non-reinnervated DIEP flap resulted similar to the values in reinnervated DIEP flap. 


\section{4}

\section{Eyelid Reconstruction with Microsurgical ALT Flap}

\author{
P. Mulas, F. Farace, A. Puddu, A. Bulla, C. Rubino \\ University of Sassari, Department of Plastic \\ Reconstructive Surgery (Head: Prof. G.V. Campus), School \\ of Specialization in Plastic Reconstructive and Aesthetic \\ Surgery (Head: Prof. C. Rubino)
}

Introduction: Full thickness reconstruction of upper and lower eyelids is an interesting surgical challenge. Microsurgical flaps may represent an option. We report a new application of ALT flap in superior and inferior homolateral eyelids reconstruction in a patient suffering for burn outcomes.

Methods: Clinical case: 65 year old man, ictus cerebri, $45 \%$ TSBA of II-III degree involving head and scalp after prolonged contact with a brazier. Patient showed wide necrosis of the left superior eyelid and superficial and deep burns to the inferior homolateral eyelid, face and controlateral eyelids; exposure keratopathy and glaucoma with left eye complete loss of the visus. Early treatment: wide dermoabrasion, full (face) and partial thickness (skull) skin-grafts. After one year: scar retraction of both left eyelids, inside epicantus, lagoftalmus, epiphora. Elevator muscle and ocular globe movements partially preserved. Surgical technique: preparation of ALT flap based on a muscle-cutaneous perforator vessel, thinning and separation to recreate rhyme and commissure of both eyelids; residual conjunctiva for inside coverage; T-T anastomosis with superficial temporal vessels and elevator muscle stump fixing to the new superior eyelid.

Results: After 6 months, there is no scar retraction that compromises the recostruction. The flap thinned with time and a little residual movement of elevator is appreciable.

Discussion: When local flaps are not available, microsurgical ones represent a reliable option. In our patient, free forearm flap was first considered, but Allen test was negative, so we try ALT that may be useful for unilateral upper and lower eyelid reconstruction in such selected situations.

\section{5 \\ Micro-Mitek Anchor System in the Fixation of Facial Soft Tissues}

\author{
M. Grieco, M. Valente, L. Annacontini, G. Giannini, \\ D.N. Massariello, G. Gozzo
}

Department of Plastic and Reconstructive Surgery University of Foggia, Italy, Head: Prof. Aurelio Portincasa, EBOPRAS Fellow

Introduction: The need to fix soft tissues to bone structures is a frequent problem in craniofacial surgery. Traumas, neoplasia or craniofacial surgery can cause the cantal ligaments to disconnect, with following palpebral dislocation, palpebral fissure change and lagophthalmia. Correcting injuries of the facial nerve and skin excess due to neurofibromatosis is another clue to soft tissues suspension.

Materials and Methods: The Mitek Anchor System (MAS), is composed of titanium microanchors $(1.8 \mathrm{~mm}$ diameter and $5 \mathrm{~mm}$ long), with nickel-titanium wings to ensure bone fixation and with a double non-reabsorbable suture thread. The microanchor screw fixing has occurred after a skin incision and the creation of a bone hole through drill. The suture thread is used for suspension. Since 2002, MAS has been used in 11 patients ( $7 \mathrm{M}$ and $4 \mathrm{~F}$ average age 57 years): in 3 patients for the correction of the facial nerve paralysis, in 2 patients suffering from neurofibromatosis for the suspension of exceeding cheek skin and in 6 patients for cantal reconstruction (2 internal and 4 external). In 8 out of 11 cases, the use of this technique has followed other procedures (1 year on average) such as reconstructions using microplates, excision of neurofibromas, etc.

Results: The suspension and the fixation of soft tissues has required $<1.5 \mathrm{~cm}$ incisions, minimal detachments and rapid surgical time. No complication has been observed. Results are stable in time (average follow-up: 2 years).

Conclusions: The various techniques proposed for the suspension of the soft tissues, although valid, need complex interventions with wound, complication risk, high learning curve and long surgical time. The use of MAS is a valid and secure option with low and stable-in-time complication risk.

\section{6 \\ Use of Propeller Flaps in Lower Limb Reconstruction: A New Approach}

\section{G. Basso, L. Annacontini, P. Bisceglia, N. Fini, A. Maiorella}

Department of Plastic and Reconstructive Surgery University of Foggia, Italy, Head: Prof. Aurelio Portincasa, EBOPRAS Fellow

Introduction: Lower limb reconstruction has always been a challenge for plastic surgeons because of the scarce availability of local flaps and for the high risk of complications in patients affected by diabetes, vascular diseases, immunodepression and low compliance. After the introduction of microsurgery, free flaps became the golden standard for lower limb reconstruction. Nowadays the development of perforator flaps, both local and microsurgical, a new surgical option has been provided, with a good versatility, reliability and quite easy to perform.

Methods: On December 2006 a male patient, R.F., aged 37, came to our Department. He had operation six weeks earlier operated for reduction and osteosyntesis of fibula fracture of the left lower limb, distal third, after a work trauma. He presented a loss of substance of abount $3.5 \times 2.5 \mathrm{~cm}$ at the $1 / 3$ distal, medially, of the lower left leg, with a fibrinous fundus, irregular margins and surrounding skin hiperemic and oedematous. The patient had good general health conditions, wasn't a smoker and didn't present main risk factors, so a local propeller flap based on perforators vassels of peroneal artery was performed, after preoperative doppler exam. The donor area has been closed with direct suture. A drainage was positioned for 4 days. The patient had short term prophylaxis.

Discussion: No major complications were observed. A delayed wound healing on the distal flap margin healed completely after 3 weeks, after topical medications. Functional recovery was fast and complete. The patient had a high compliance.

Conclusions: The usage of local flaps based on perforator vessels (propeller flaps) represents a good reconstructive option and, 
when it's possible, a valid alternative to free flaps. Popeller flaps give the advantage of reduced operative time, a fast postoperative recovery and, as a conequence, a higher patients' compliance. Moreover we have a reduced morbility of donor site, with the preservation of muscular tissue and main vessels, with a valid reconstructive, functional and aethetic result. The learning curve too is reduced if compared to the microsurgical techniques used in this kind of reconstructions.

267

\section{Learning Curve for the Identification of Sentinel Node in Melanoma}

\section{G. Giannini, M. Grieco, L. Annacontini, A. Campanale, G. Basso, A. Maiorella}

Department of Plastic and Reconstructive Surgery University of Foggia, Italy, Head: Prof. Aurelio Portincasa, EBOPRAS Fellow

Introduction: Sentinel node biopsy shoes the first node draining melanoma site by Tc 99 perilesional injection; it also permit the node surgical excision by the use of intraoperative gamma-camera. The greatest sensitive and the very little invasivity make this methods the gold standard in a adeguate selection of melanoma patients to undergo linphadenectomy. But this methods needs a training period. The authors show the results of their self-test by learning curve in period of 5 years.

Materials and Method: In the 2006, 3 residents, with the same tutor over view, at its autotest by learning curve (STATVIEW) in the permaning sentinel node biopsy technique.

The eam study whose:

-node surgical identification capability

-number of surgical intervention

-time per intervention

-learning curve at T0-T6-T12 months

-node basing site: groin or axillar.

Results: Surgical identification capability of the node was $20-40 \%$ in the first 6 month and $80-100 \%$ at the end of 12 months. Success rate in the sentinel linphonode procedure at the level of groin region past from $0-20 \%$ at the end of 3 months to $80-100 \%$ at the end of 12 months. Success rate at the level of axillar region past from $0-15 \%$ at the end of 3 months to $70-100 \%$ at the end of 12 months. The time for to found the sentinel linphonode past from 45' at the first 3 months to 30' at the 6 months and to 20' at the end of 12 months for the two regions (axillar and groin). Biside the timing of the procedure progressively decreased with the increasing in number of procedure for each surgeon (surgeon 1:12 patients in 1 year; surgeon 2:7 patients in 1 year; surgeon 3:10 patients in 1 year).

Conclusion: Despite, this technique easly perfomed and adeguate training is fundamental for the resident in training, because the sentinel linphonode technique is one of the basic knowledge that the Plastic Surgeon should have.

\section{8}

\section{Conventional Microsurgical Techniques vs. VAC Therapy in the Reconstruction of Inferior Limb Defects}

\author{
L. Annacontini, A. Campanale, M. Grieco, M. Valente, \\ G. Gozzo \\ Department of Plastic and Reconstructive Surgery - \\ University of Foggia, Italy, Head: Prof. Aurelio Portincasa, \\ EBOPRAS Fellow
}

Introduction: Functional and aesthetic reconstruction of the inferior limb with a stable, sensate soft tissue integument after complex posttraumatic or oncological defects is demanding. The reconstructive approach has to consider both patient profile and the reconstructive tree, with free microvascular flaps playing a primary role. Nevertheless new interesting devices are facing the reconstructive problem as valid alternative option to the classical approach. Among these, the Vacuumassisted closure $\left(\operatorname{VAC}^{\mathbb{R}}\right)$ therapy is applied to an open wound to create subatmospheric pressures within a sealed dressing. The therapy increases blood flow, decreases edema and bacterial count, and promotes the formation of granulation tissue. Authors compare the two methods in solving moderate to huge defects of the inferior limb.

Materials and Methods: From January 2000 to January 2007, $42 \mathrm{pz}(31 \mathrm{M}, 11 \mathrm{~F})$ underwent inferior limb reconstruction. $28 \mathrm{pz}$ were reconstructed with microsurgical procedures ( 8 latissimus dorsi flap, 12 chinese flaps, 3 rectus abdominis flaps, 5 lateral arm flaps) and 14 were previously treated with two to four cycles of VAC ${ }^{\circledR}$ therapy at 75 to $150 \mathrm{mmHg}$, both in continuous and intermittent modality plus skin graft. Mean defects sizes were $15 \times 10 \mathrm{~cm}, 30 \%$ of them deep to the bone, $70 \%$ to the muscle. $67 \%$ of the patients were treated in emergency, $31 \%$ underwent elective surgery. Adeguate pre-op selection was mandatory in all cases.

Results: All pz treated gained satisfactory results from the functional point of wiev. Aesthetical results in $64 \%$ of the cases were poor but the aim was the solution of a large defect often in emergency; in this evenience microsurgery represented the only valid option, offering valid solutions to all type of defects (large, deep, potentially infected, etc.). VAC $^{\circledR}$ therapy, on the other side, solved with a 'mild' approach also compromised situations not in emergency. The necessity of a skin graft after the application of the $\operatorname{VAC}^{\circledR}$ had the disadvantages to prolong the hospital stay. Microsurgical harvesting of a flap left a visible scar in the donor site both with a functional impairment depending of the flap chosen.

Conclusion: Both microsurgery and $\mathrm{VAC}^{\circledR}$ therapy, applied to post traumatic and oncological defects, allow the solution of moderate to huge defects. Patients selection is mandatory in both cases. Microsurgery involve skilled surgeons with long experience especially in emergency cases. VAC ${ }^{\circledR}$ therapy can easily be applied also by novices. We retain they are two valid weapons in surgeon hands. 


\section{9}

\section{Improving of the Outcome in Patients with Carpal Tunnel Syndrome: A New Protocol with Gliding Hyaluronic Acid Gel}

S. Scalseggi, L. Annacontini, D.N. Massariello,

A. Campanale, A. Maiorella

Department of Plastic and Reconstructive Surgery, University of Foggia, Italy, Head: Prof. Aurelio Portincasa MD, EBOPRAS Fellow

Background: Carpal tunnel syndrome (CTS) is the most common compression syndrome of the upper extremity. Surgical incision of the transverse ligament is still the best option in stage II patients. Recurrence rate is up to $20 \%$.

Aims: Authors present their experience in the use of hyaluronic acid gel (Hyaloglide ${ }^{\circledR}$ ), a cross-linked, gliding, biocompatible and bio-resorbable gel. It provides an anti-adhesive environment in the first weeks improving the surgical outcome.

Patients and Methods: From June 2004 to December 2006, 60 patients ( $44 \mathrm{~F}$ and $16 \mathrm{M}$ ), range 14-66 yrs old, with STC, entered in our single blind study. They were divided in two randomized groups: A (CONTROL) and B (Hyaloglide ${ }^{\mathbb{R}}$ ), homogenous for age, sex, associated pathology, length and course of median nerve compression. Both groups underwent on a one-day surgery, local anesthesia, tourniquet, mini incision, section of the transverse ligament, removal of a little strip of it. In the group B Hyaloglide ${ }^{\circledR}$ was applied before skin suture. Follow up was carried on every 15 days for 6 months by QUICK DASH, BMRC and VAS tests, to evaluate pain, functional assessment, sensibility recovery, recurrence rate.

Discussion: The results were statically evaluated. In both groups pain and dishestesia disappeared 24 hours after surgery. Return to daily activities: group A in 12 days; group B in 7 days. Return to working activity: group A in 21 days; group B in 15 days. All evaluated tests showed better results in group B until day 45; then, differences between the two groups decreased, overlapping by the 60 th day. No recurrence was observed.

Conclusions: Hyaloglide ${ }^{\circledR}$ is a valid support especially in the first weeks immediately after surgery. Clinically, our protocol ensure a better compliance and earlier recovery of the patient in which the gel was employed. The study is still running on, to increase the number of treated patients.

\section{Breast Surgery}

270

\section{Day Surgery (DS) Model for Sentinel Lymph Node Biopsy (SLNb) in Breast Cancer by means of Wireless Gamma Probe (WGP)}

\author{
C. Bertoglio, A. De Luca, C. Valli, C. Perrucci, \\ I. Scandroglio \\ Az. Ospedaliera di Busto Arsizio, Tradate
}

Introduction: ALMANAC trial stated that SLNb should be the treatment of choice for early-stage breast cancer and clinically negative nodes. Several studies proved the feasibility of this procedure under local anaesthesia (LA).

Methods: DS approach for SLNb has been investigated from Jan 07. Patients were selected according to FONCaM guidelines. Preoperative lymphoscintigraphy mapping (PLM) was routinely done to define nodes localization at least 18 hours before surgery. After LA (2\% Ropivacaine) and subcutaneous injection of patent blue dye SLN intraoperative detection was carried out by WGP and direct vision.

Results: 21 outpatients underwent SLNb with no failure in detection and good correspondence to PLM. No discomfort was recorded and all patients were discharged 8 hours after procedure. Follow-up during next 48 hours showed no morbidity and patients underwent radical surgery within 2 weeks. Histological findings on retrieved specimens revealed tumor infiltration in 5 cases $(3 \mu$ metastasis) and deserved further axillary dissection.

Discussion: Our preliminary experience shows that DS model adds further advantages in terms of QOL and patient satisfaction and should be preferred to perform SLNb under LA. Gamma Finder is the only cordless hand-held GP available enabling easy and accurate examinations. Better knowledge of LNstatus avoid false negatives of intraoperative evaluation and loss of $\mu$ metastasis.

\section{1 \\ New Option in Non Palpable Lesions (NPLs) of the Breast: Our Experience with Mammotome ${ }^{\circledR}$ System}

\author{
G. Limite, D.P. Calabrese, G. Di Martino, E. Esposito, \\ A. Farina, G. Garofalo, E. Palma, E. Villamaina, C. Formisano \\ Dipartimento di Chirurgia generale, Università degli Studi \\ di Napoli Federico II
}

Introduction: Screening mammography has allowed diagnosis of malignant lesions in preclinical stage, favouring mortality rate reduction and better conservative surgical techniques. The aim of our study is to value the accuracy of two diagnostic procedures in the management of NPLs of the breast: open biopsy and micro-histological biopsy with Mammotome. 
Methods: Data were collected about 182 patients with NPLs treated at our department from 2001 to 2005. 94 women $(52 \%)$ received Mammotome biopsies, while 88 women (48\%) were directly treated with open biopsies. 20 women of those treated with Mammotome (16 with an histological diagnosis of cancer or severe atypias and 4 with an inconclusive histology but with a suspicious mammography) were operated. Finally 108 women were surgically treated.

Results: Histological findings of women at first treated with Mammotome core-biopsy and then operated revealed: 14 breast cancers, 5 fibrocystic diseases and 1 atypical ductal hyperplasia. Finally, 62 patients (34\%) had a diagnosis of breast cancer. Nowaday we have noted only 3 breast cancer recurrences.

Discussion: Micro-histological biopsy with Mammotome represents a valid procedure in the integrated diagnosis of NPLs of the breast and, at the same time, an efficient complement to open biopsy when there is indication for surgery.

\section{2}

\section{Nipple Sparing Mastectomy: An Initial Experience}

\section{T. Fogacci, D. Samorani \\ Ospedale Infermi Rimini}

Introduction: The nipple-sparing mastectomy (NSM) is a technique of mastectomy and conservation of the nipple-areola complex (NAC). A lot of studies showed that in many cases is possible conserve the NAC because it is not invade from that cancer. In some center in sperimental cases this technique is used.

Material and Methods: We performed in our center 29 cases of NSM in 28 patients in the last 2 years. Always we made the intraoperative histological examination of the tissute under the NAC. In 1 cases it was positive and we transformed the NSM in to Skin Sparing Mastectomy. In 1 case the definitive histological exam showed the invasion of the tissute under the NAC (made negative during the operation) and we performed the asportation of NAC in local anestesia. In all the cases we put a skin expander under the muscle. In all the patients was performed radiotherapy on the NAC after the operation.

Results: All the patients are alive without local or distant recidive at a median follow-up of 10 months. The definitive prosthesis were put in 10 patients. In 20 cases we observed a nipple deviation, very important in 10 cases to be necessary an intervent of NAC translation (in local anesthesia).

Discussion: The NSM is feasible and sure if the indications are good. The vantage are a better esthetic results and a very important psychological impact, but at the state of the art the NAC is a sperimental technique useable in selected, specifical center.

\section{3}

\section{Metachronous Paget Breast Disease: Case Report}

\author{
G. Iside, A. Gubitosi, G. Moccia, F.A. Malinconico, \\ D. Parmeggiani, F. Gilio, C. Cognetti, F. Foroni, \\ P. Sperlongano, M. Agresti \\ $X$ Divisione di Chirurgia Generale e Fisiopatologia \\ Oncologica - AUP - SUN, Napoli
}

Introduction: Paget breast disease is a kind of intraductal carcinoma that through an intracanalicular diffusion invades the basal epidermical layer, reaching the areola and nipple, producing a typical erythematous desquamative eczematous-like lesion. This neoplasia can remain undetected for a long time and inadequately treated as a dermatological affection. Synchronous or metachronous lesions are very uncommon. Surgical choice is conditioned by the presence of a tumor below the epidermical lesion, by its dimensions, and by the possible lymph node involvement.

Methods: Our case refers to a 73-year old woman who came to our clinic for an erythematous microulcerated lesion extended to the nipple, the areola and on the periareolar skin of the left breast. This lesion was seven years old and it had enlarged in the last two months. The patient underwent skin biopsy that revealed 'invasive ductal undifferentiated Paget-associated breast carcinoma'. Given these facts, the patient underwent a radical mastectomy with third level lymph node dissection (Madden).

Results: The postoperative course was complication-free and the histopathological analysis on the resected breast confirmed that found on biopsy. The final diagnosis was intraductal carcinoma with residual Paget focus, with metastasis-free regional resected lymph node.

Conclusion: Surgical therapy can be radical or conservative. From our experience we think that lesion biopsy is always necessary to formulate a correct diagnosis to schedule an appropriate therapeutic approach.

\section{4 \\ Learning Curve for the Sentinel Lymph Node Biopsy - Analysis of the Rome Breast Cancer Study Group}

\author{
S. Drago ${ }^{1}$, R. Piccirillo ${ }^{2}$, A. Bellotti ${ }^{1}$, E. Mastrodicasa ${ }^{3}$, \\ G.B. Grassi ${ }^{1}$ \\ ${ }^{1}$ Chirurgia Generale ed Oncologica - Ospedale San Filippo \\ Neri - Roma; ${ }^{2}$ Chirurgia senologica e Servizio di \\ Senologia - Policlinico Militare 'Celio' - Roma; \\ ${ }^{3}$ Dipartimento di Anestesia e Rianimazione - Università \\ degli Studi di Roma 'Tor Vergata' - Roma
}

Introduction: Sentinel node biopsy is now a widely accepted technique for axillary staging in patients with breast cancer. Identification rate (IR) and false negative rate (FN) of the sentinel node represent well recognized quality indicator for this technique.

Materials and Methods: Aim of this study is to evaluate the identification rate with reference to case volume and specialization of different surgeons. Computerized database of seven Breast Unit in the 
Rome area have been reviewed and identification rate for any involved surgeon in each hospital has been analysed and compared to the case volume and the learning curve for all surgeons.

Results: In the total group of 1,689 patients IR has been higher than $90 \%$ in each hospital. Significant differences have been found between surgeons (75-99\%) and differences in outcome have been related to the surgeon's case volume and learning process.

Discussion: Sentinel node biopsy is a simple and feasible technique, but still has to be considered a highly specialized technique as better results are obtained by dedicated surgeons with high case volume.

\section{5}

\section{Sentinel Lymph Node Biopsy Combined with Radioguided Occult Lesion Localisation in Non-Palpable Breast Tumors}

\author{
N. Rocco, C. Rispoli, M. Celiento, L. lannone, V. Girardi,
} B. Amato

Department of General Surgery, University 'Federico II' of Naples

Introduction: Sentinel lymph node biopsy (SLNB) is widely used for staging breast cancer. SLNB accurately determines axillary lymph node status with a low false negative rate. The aim of this study was to evaluate the efficacy of radioguided occult lesion localisation (ROLL) for non-palpable invasive breast cancer combined with sentinel lymph node biopsy (SLNB) and to compare the amount of tissue excised by radioguided investigation versus the hook-wire technique.

Methods: We injected $45 \mathrm{MBq}$ of radiolabelled Technetium intratumorally and $15 \mathrm{MBq}$ subdermally 18 hours before surgery in twelve women with US-FNAC verified non-palpable breast cancer. We identified by gamma probe non-palpable tumours, which were excised followed by identification and excision of the sentinel lymph node. We compared our results with a group of 12 women with nonpalpable lesions marked by hook-wire localisation.

Results: ROLL combined with SLNB was successful in 100\%; the difference of excised tissue in both group was not statistically significant.

Discussion: ROLL combined with SLNB is technically possible and safe, resulting in minimisation of the surgical intervention and a decrease in postoperative morbidity. ROLL is more precise than the hook-wire procedure even though the amount of tissue excised was the same in both groups.

\section{6}

\section{Sentinel Lymph Node Biopsy for Ductal Carcinoma in situ: Is it Useful?}

N. Rocco, C. Cirillo, L. De Magistris, A. Braun, D. De Vito, R. Compagna

Department of General Surgery, University 'Federico II' of Naples

Introduction: Intraductal carcinoma of the breast (DCIS), by definition, cannot give axillary metastases. Axillary dissection is therefore not indicated. The role of the sentinel node biopsy in the management of DCIS has not yet been estabilished. A 6-13\% risk of SLN involvement is reported in literature. The aim of this study is to assess the role of SLN biopsy in patients with DCIS.

Methods: From December 2005 to June 2007, 48 consecutive patients with DCIS of the breast underwent SLN biopsy in our Department. Clinical and pathological data were prospectively collected. Cases with microinvasion were excluded from this study. Lymphatic mapping was performed using a radiocolloid technique. All the patients underwent conservative surgery and removal of the SLN which was sent for conclusive histology.

Results: SLN metastases were detected only in one out of 48 (2.1\%) patients. In another patient only micrometastasis (less than $2 \mathrm{~mm}$ ) was detected. The patient with SLN metastases underwent complete axillary dissection and no additional positive axillary lymph nodes were found.

Discussion: According to our data, due to the low prevalence $(2.1 \%)$ of metastatic involvement, SLNB should not be considered a standard procedure in the treatment of all patients with DCIS. SLNB should be considered in case of DCIS where there exists a strong doubt of invasion at the definitive histology.

\section{7}

\section{Axillary Lymphadenectomy for Breast Cancer: Use of Fibrin Glue to Reduce Sieroma}

\author{
G. Docimo, R. Ruggiero, S. Gili, E. Irlandese, F. lovino, \\ F. Lo Schiavo
}

Second University of Naples - Surgical Department

Background: Axillary lymphadenectomy remains an integral part of breast cancer treatment for prognostic and curative purposes. There is still a significant incidence (15-81\%) of complications associated with axillary lymphadenectomy, including lymphorrhea, lymphoceles and in rare cases lymphedema (swelling of the arm). It is possible to avoid axillary dissection in selected patients (T1 N0) using the sentinel lymph node technique. However, in all cases where $\mathrm{T}>3 \mathrm{~cm}$ and in patients with $\mathrm{T} 1 \mathrm{~N} 1$ or with metastatic sentinel lymphnode, conventional axillary lymphadenectomy remains the primary surgical protocol for quadrantectomy and mastectomy. Among methods employed to reduce seroma magnitude and duration, fibrin glue has been proposed in numerous studies with controversial results.

Methods: Sixty patients underwent quadrantectomy or mastectomy with level I/II axillary lymphadenectomy; a suction drain was fitted in all patients. Fibrin glue spray were applied to the axillary fossa in 30 patients; the other 30 patients were treated conventionally.

Results: Suction drainage was removed between post-operative days 3 and 4 . Seroma magnitude and duration were significantly reduced $(\mathrm{p}=0.004$ and 0.02 , respectively), and there were fewer evacuative punctures, in patients receiving fibrin glue and collagen patches compared with the conventional treatment group.

Conclusions: Use of fibrin glue does not always prevent seroma formation, but does reduce seroma magnitude, duration and necessary evacuative punctures. 


\section{Experimental Surgery}

278

\section{Transvaginal Access for Laparoscopic Cholecystectomy}

\author{
W. Bugiantella, L. Graziosi, P. Rossi, A. Donini
}

Department of General and Emergency Surgery, University of Perugia, S.M. della Misericordia Hospital,

Perugia, Italy

Introduction: Natural orifice transluminal endoscopic surgery (NOTES) is arising as an alternative access to the peritoneal cavity that eliminates abdominal incisions and incision-related complications by combining endoscopic and laparoscopic techniques.

Methods: From May 2007 we started to perform the transvaginal access for laparoscopic cholecystectomy. After pneumoperitoneum induction we cut the posterior vaginal fornix and introduce the operative endoscope into peritoneal cavity under laparoscopic control. The placement of one trocar is useful to insufflate $\mathrm{CO}_{2}$, to dissect and clip of the structures of Calot's triangle and to retrieve the gallbladder. All the principles of laparoscopic cholecystectomy are strictly respected.

Result: We are experimenting that the transvaginal access is technically feasible, safe, it enables a very good visions of the peritoneal cavity even working with a low pneumoperitoneum pressure (6-8 $\mathrm{mmHg}$ ) and ensures less postoperative pain.

Discussion: Theoretical advantages of NOTES over the laparoscopic approach include a reduction in postoperative abdominal wall pain, wound infections, hernias and adhesions formation, and a better aesthetic result. Despite the exciting capabilities of NOTES, several key issues need to be characterized before NOTES becomes a routine practice, such as technical challenges in developing a new platform and new devices for creating and safely closing the viscerotomy and for manipulating intraabdominal organs. We believe that NOTES is not only a challenge but the beginning of a new surgical endoscopic era.

\section{9}

\section{Improved Secretory Function of Small Pancreatic Islets Generated by Reaggregation in Hanging Drop Cultures}

G. Cavallari1,3, R. Zuellig ${ }^{2}$, R. Lehmann², B. Nardo ${ }^{3}$, A. Faenza ${ }^{3}$, A.D. Pinna ${ }^{3}$, M. Weber ${ }^{1}$, W. Moritz ${ }^{1}$

${ }^{1}$ Clinic for Visceral and Transplant Surgery, Department of Surgery, ${ }^{2}$ Division of Endocrinology and Diabetes, Department of Internal Medicine, University Hospital Zurich, ${ }^{3}$ Department of Surgery, Intensive Care and Transplantation, S.Orsola-Malpighi Hospital, University of Bologna, Italy

Introduction: Recently we reported a negative relationship of graft function and islets size concluding that small islets are superior to large islets. Islets can be dissociated into single cells and reaggregated into so called 'pseudoislets', which are functionally equivalent to intact islets. Aim of our study was develop a technique that provides pseudoislets of defined, small dimensions.

Methods: Islets were dissociated into single cells by trypsin. Fractions with defined cell numbers were seeded into single drops onto cell culture dishes which were incubated for 4-6 days. Newly formed pseudoislets were analysed for dimensions, morphology, cellular composition and glucose stimulated insulin secretion (GSIS).

Results: Morphology, cellular composition and architecture of reaggregated islets were comparable to intact islets. GSIS of small pseudoislets, composed of 250 islet cells, was increased by 2.7 -fold $(\mathrm{p}<0.001)$ when compared to freshly isolated islets. In small pseudoislets, insulin secretion at high glucose was stimulated by 10.6fold, while GSIS in intact islets was enhanced only by 2.9 -fold $(\mathrm{p}<0.001)$.

Discussion: With 'hanging drop' culture method pseudoislets of standardized size and regular shape can be generated who exhibit a markedly improved insulin secretory capacity. The concept of small pseudoislet could foster new approaches in islet cell replacement therapy.

\section{0}

\section{Surgical Colic Congestion in a Constipation Model in Rats}

M. Tsivian1, F. Neri1, E. Bianchi', G.L. Piras ${ }^{1}$, G. Cavallari', R. Bertelli', P. Beltempo ${ }^{1}$, M. Pariali ${ }^{2}$, R. Aldini', B. Nardo ${ }^{1}$

${ }^{1}$ Dipartimento di Discipline Chirurgiche, Rianimatorie e dei Trapianti, ${ }^{2}$ Centro di Ricerca Biomedica Applicata (CRBA), Università di Bologna

Introduction: Conservative treatment in chronic constipation is not always successful. Surgery is indicated in severe cases, but complication rate may be high. In this study we evaluate a novel surgical procedure: distal venous colic congestion in a rat model of constipation.

Materials and Methods: 16 SD rats were assessed in 3 subsequent phases of 6 days each: phase A-normal conditions, B-loperamide-induced constipation and $\mathrm{C}$-constipated treated with colic vein legation. We analyzed daily water and food intake, feces (total and single pellet) weight (FW) and water content (WC). Histological analysis and blood biochemistry were performed in all phases.

Results: No significant hematochemical changes were observed. FW (both total and single pellet) and WC decreased in phase B and increased in phase $C(p<0.05)$. Histology showed significant difference in goblet cells population: $172 \pm 2,144 \pm 2$ and $211 \pm 2$ cells/hpf in phases A, B and C respectively ( $<<0.0001)$. Goblet cells proliferation seems to be related to modest mucosal oedema induces by the procedure.

Discussion: The surgical procedure described is safe and easily performed. Further studies are necessary to confirm our results and assess clinical applicability of this technique. 
281

\section{Endolymphatic Immunotherapy in Inoperable Hepatocellular Carcinoma}

\author{
R. Bertelli', B. Nardo', C. De Vinci' ${ }^{2}$, P. Beltempo', \\ G. Cavallari', F. Neri', M. Tsivian'1, L. Puviani', G. Pizza \\ ${ }^{1}$ Dipartimento di Discipline Chirurgiche, Rianimatorie e dei \\ Trapianti, ${ }^{2}$ Modulo Immunoterapia, U.O. Urologia, \\ Università di Bologna
}

Introduction: We report the preliminary results of endolymphatic immunotherapy in patients with inoperable hepatocellular carcinoma (HCC).

Materials and Methods: From 2003 to 2005 we enrolled 31 patients with inoperable HCC. The patients underwent monthly endolymphatic injections of $15-30 \times 10^{6}$ IL-2-activated peripheral autologous lymphocytes (LAK) and $250 \mathrm{U}$ of IL-2. Follow up included blood biochemistry every 3 months and imaging studies every 6 months. To assess therapy efficacy we considered 12 biochemical parameters, vascular invasion or thrombosis, Child-Pugh, histological grading, lymphoadenopathy, viral state and alpha-fetoprotein.

Results: 16 patients completed at least 3 cycles and 10 patientsmore than 6. No clinically significant adverse reactions occurred. Imaging studies showed no significant decrease in tumor mass. However, the survival of patients who completed 12 therapy cycles was significantly higher than survival of patients with less than 12 cycles. Both are significantly higher than that of untreated patients. All patients with 12 completed cycles showed an improvement of 9 parameters or more.

Discussion: Endolymphatic immunotherapy is safe, easily performed, cheap and effective in terms of survival. This study should encourage future large scale studies in order to reach a firmer conclusion and define uniform inclusion criteria.

\section{2 \\ Effects of VEGF on Synthetic Arterial Grafts. An Experimental Study}

\author{
G. Cavallaro, A. Polistena, B. Randone, A. Cucina,
}

C. Paparelli, G. De Toma, A. Cavallaro

Department of Surgery 'P. Valdoni', Policlinico Umberto I, University 'La Sapienza', Rome

Background: Lack of endothelialization and abnormal smooth muscle cell (SMC) growth adversely affect the outcome of vascular synthetic grafts. The aims of our study were to investigate how a coating of extracellular matrix (ECM) and vascular endothelial growth factor (VEGF) might affect the endothelialization rate, smooth muscle cells (SMC) proliferation, and myointimal hyperplasia in experimental arterial ePTFE grafts.

Methods: In each of 30 male Lewis rats, a 1-cm-long ePTFE graft was inserted at the level of the abdominal aorta. Animals were randomized in five groups (six animals each): groups A and A1 received ePTFE grafts coated with a synthetic extracellular matrix (growth factor-reduced matrigel) containing VEGF; groups B and B1 received $\mathrm{PPTFE}$ grafts coated with synthetic ECM; and group $\mathrm{C}$ received ePTFE grafts alone. The grafts were explanted at 30 days from surgery for immunohistochemical analysis.

Results: Both endothelialization rate and myointimal hyperplasia were augmented in group A versus groups B and C, and these findings were statistically significant. SMC density resulted significantly higher in group A versus groups $\mathrm{B}$ and $\mathrm{C}$, and this was associated with an altered expression of bFGF and TGFbeta.

Conclusions: Pretreating ePTFE grafts with synthetic ECM and VEGF results in better endothelialization, but also in undesired higher SMC density and myointimal hyperplasia.

\section{3}

\section{Three Steps to Achieve Microsurgical Skills in Reconstructive Microsurgery}

\author{
E. Cigna, G. Bistoni, F. Conte, E. Trignano, C. Monarca, \\ M.G. Onesti \\ Department of Plastic Surgery, University of Rome - La \\ Sapienza
}

Introduction: Today, microsurgery plays a fundamental role in plastic surgery. The rat model has been, and still is, the standard model for microsurgery training, but stringent laws and the rising costs of this model have necessitated alternative models. To highlight a suitable option in training we developed a three-step approach. This method is currently used to teach residents to enter the arena of microsurgery.

Methods: The first step uses a non-animal model such as silastic tubes and latex gloves. The second step uses on a non-live animal model, such as chicken legs and wings. The third step is based on a fresh human-tissue specimen obtained from the operating room, which would otherwise be discarded.

Results: The first step has the aim to acquire the knowledge of sutures, handling instruments and the microscope, and to learn the way to tie sutures. The second one is essential to acquire the skills of the anastomotic technique The third step is essential to acquire the skills of adventectomy and vessel harvest, as well as to practice microsutures in human vessels and nerves.

Discussion: This three-step technique allows the training of basic microsurgical skills, with specific advantages of enabling practice of all steps, which is not possible on synthetic models and enables the trainee to learn all the basics of microsurgery.

\section{4}

\section{'The Adhesion Power of Different Prosthetics Materials for Hernia Surgery Implanted in Peritoneal Peritoneum'}

\author{
F. Gazzotti', L. Ansaloni ${ }^{1}$, F. Catena', F. Coccolini', \\ S. Di Saverio', M. Fini², S. Gagliardi', R. Giardino², A.D. Pinna \\ U.O. Chirurgia d'Urgenza, 9 - Policlinico S. Orsola- \\ Malpighi, Bologna
}

Although for abdominal wall defects repairs polypropilene (PP) is the prosthetic material mainly used, its potential role in adhesion 
formation. Aim of our work is to test the adhesions' formation to different prosthetic materials when implanted intraperitoneally. In our study we compare the adhesion formation due to PP with three other kind of composite prosthesis (PP + ePTFE, PP + Surgisis, $\mathrm{PP}+$ Polyuretan) and a group of control. The animals were sacrificed at 21 days, 3 and 6 months ( 4 at each time) following surgery and analyzed for the presence of adhesions. The adhesions were scored macroscopically in terms of extent (quantity) and type (quality) by two independent observers. Although PP + ePTFE, PP + Polyuretan and $\mathrm{PP}+$ Surgisis meshes appear to provide a better alternative to the use of PP meshes for intraperitoneal implant in terms of the formation of adhesions, PP + Polyuretan and PP + Surgisis meshes seem significant less adhesiogenic than PP + ePTFE.

\section{Thoracic Surgery}

\section{5 \\ Surgical Management of Tuberculosis: Our Three Year Experience}

\section{Loizzi², A. De Palma', N.P. Ardòn', A. Pernice², F. Sollitto ${ }^{2}$ \\ ${ }^{1}$ Cattedra di Chirurgia Toracica, Università degli Studi di Foggia; ${ }^{2}$ Cattedra di Chirurgia Toracica, Università degli \\ Studi di Bari}

Introduction: In this review of tuberculosis cases we analyzed indications and methods of surgery, results, perioperative complications and postoperative treatment.

Methods: We reviewed records of 27 patients (18 males, 10 females), mean age 53 years, who underwent surgical procedures for tuberculous disease between February 2004 and January 2007 at our Institution. Tuberculosis presented as: pulmonary nodules $(n=7)$, infiltrates $(n=4)$, cavities $(n=1)$, relapsing pleural effusion $(n=4)$, chronic empyema $(n=5)$, chest wall tuberculosis $(n=3)$, sternoclavicular joint tuberculosis $(\mathrm{n}=1)$, cervical lymphadenitis $(n=2)$. At sputum microbiological tests, there were 4 bacillary cases.

Results: We performed: lobectomy $(n=2)$, wedge resection $(\mathrm{n}=8)$, pneumotomy $(\mathrm{n}=2)$, thoracoscopic pleural biopsy and drainage $(n=4)$, decortication $(n=5)$, open biopsy $(n=4)$, cervical lymphadenectomy $(\mathrm{n}=2)$. Perioperative complications were: bleeding $(n=2)$, prolonged air leaks $(n=3)$, pneumothorax $(n=1)$. Chemotherapy associated isoniazid, rifampicin, ethambutol, pyrazinamide.

Discussion: The incidence of tuberculosis has recently increased due to AIDS epidemic and third world immigration. Early diagnosis is crucial for interrupting disease transmission. In our experience surgery results a valid contribution for diagnosis and/or therapy of pulmonary and extra-pulmonary tuberculosis, with a broad spectrum of indications.

\section{6}

Surgical Treatment of Thoracic Empyema in HIV-Infected Patients with VATS Approach

\author{
L. Bertolaccini, V. Della Beffa, A. Berra, E. Manno, \\ F. Massagglia \\ Ospedale Maria Vittoria - Chirurgia Generale e Toracica, \\ Torino
}

Introduction: Empyema causes in HIV high morbidity and mortality. Risk factors include low CD4 ( $<200$ cells/l); IV-drug abuse; lower respiratory tract infections.

Materials: Between 2001-2006, 15 HIV patients (13 men, 2 women) were admitted with empyema treated by antibiotics prior surgery. Indications for surgery included empyema and: fever after conservative management, multiloculated pleural effusion, or bronchopleural fistula. All patients underwent VATS drainage and decortication. Decision to perform a resection (wedge or lobectomy) was based on presence of lung necrosis.

Results: Mean age was 39.9 years. Mean time of HIV-diagnosis was 3.8 years. $50 \%$ of patients had history of Pneumocystis jiroveci pneumonia (PJP), 30\% had history of tuberculosis (TB). Patients were divided into two groups based on CD4. Total CD4 $<200$ Cells/1 Patients 157 (46.7\%) Lung abscess 10 (66.7\%) 6 (85.7\%) Wedge resection/lobectomy $6(40 \%) 5(83.3 \%)$ Chest tube removal (days) 5.4, 6.9 Complications $4(26.7 \%) 6(85.7 \%)$. There were no deaths within 60 days after surgery. Most frequent pathogens were Grampositive bacteria; 4 patients $(26.7 \%)$ had negative culture findings.

Discussion: HIV with PJP, TB, and low CD4 are at increased risk of VATS treatment of empyema. Surgical treatment of empyemas in HIV with CD4 counts $>200$ cells/l can be treated safely and successfully with VATS.

\section{7 \\ Management of Suspected Tracheobronchial Inhaled Foreign Bodies in Infancy}

\author{
S. Conforti, A. Rinaldo, S. Fieschi, M. Torre \\ Chirurgia Toracica, Azienda Ospedaliera Niguarda Ca' \\ Grande - Milano
}

Introduction: Foreign body aspiration (FBA) in infancy is a common and potentially life threatening event. Undiagnosed or delayed removal can lead to serious morbidity. We describe our experience about diagnosis and management of suspected FBA in infants.

Methods: We reviewed all cases underwent FOB for suspected FBA in our Department from January 2000 to July 2007. History, signes, symptoms and radiological findings were retrospectively analyzed.

Results: 75 young patients ( 8 months- 3 years) underwent FOB for suspicion of FBA. In 11 patients FOB was negative but in 6 cases of these undirect signes of previously inhaled foreign body were present. In all the remaining 64 patients different kind of FB were detected. Nuts and almonds were extracted in 51 patients. Rigid bronchoscopy and general anesthesia was considered mandatory in all cases for endoscopical removal. 12 patients in which diagnosis was 
delayed after two weeks needed prolonged duration of procedure and rebronchoscopy after one months for definitive treatment. In one case of inhaled open safety pin, bronchoscopy failed to remove and lower lobectomy was necessary. In all other cases complete removal of FB was achieved without mortality or serious complications.

Conclusions: In our experience FOB was effective and safe in diagnosis while rigid bronchoscopy and careful general anesthesia must be considered mandatory for removal.

\section{8}

\section{Surgical Treatment of Non Malignant Tracheal Lesions: A Single Center Experience}

\author{
N. Daddi', M. Urbani², A. Semeraro', A. Lomonaco', \\ R. Capozzi ${ }^{1}$, P. Roberta ${ }^{1}$, V. Tassi ${ }^{1}$, M. Sciamannini ${ }^{1}$, \\ F. Puma², G. Daddi ${ }^{1}$ \\ ${ }^{1}$ A.F.O.I. S.C. Chirurgia Toracica, Ospedale S. Maria della \\ Misericordia, Università degli Studi di Perugia, Perugia, \\ ${ }^{2}$ A.F.O.I. U.O. Chirurgia Toracica, Ospedale S.Maria, \\ Università degli Studi di Perugia, Terni
}

Introduction: Different etiologies are responsible for a wide spectrum of tracheal injuries. Some of these, such as cicatricial laryngo-tracheal stenosis and tracheoesophageal fistula (T-E) not rarely represent a challenge in tracheal surgery. Aim of this study is to retrospectively review the pattern of post-intubation lesions, extension of surgery and long term results in 49 patients.

Methods: From December 1987 to February 2007, 49 patients (23 males and 26 females), mean age 50 (range 15-78), were submitted to surgical treatment: cicatricial tracheal stenosis (Group A), T-E fistula (Group B), and tracheal tear (Group C). All patients were symptomatic.

Results: No post-operative deaths. In Group A $(n=34)$ the stricture was considered moderate (residual lumen:30-50\%) in 27 patients, and severe $(<30 \%)$ in 7 . Tracheal resection and reconstruction (RR) was performed in 24 cases, laryngo-tracheal RR in 10. Two major complications were observed (partial dehiscence of the suture). In group B $(n=10)$ : all patients underwent tracheal RR and direct closure of the T-E fistula. One patient died 10 days postop; 1 with minimal recurrence of the T-E fistula was treated by a temporary Ttube. In group $C(n=5): 3$ patients (tear of the membranous wall) were submitted to immediate tracheal suture (right thoracotomy) protected with an intercostal-muscle flap. A trans-tracheal approach trough cervico-sternotomy was adopted in one case. One patient (small sub-cricoid tear) was treated in a conservative way.

Discussion: Good surgical results in the treatment of nonneoplastic tracheal lesions are based on: (1) patient selection; (2) timing of surgery; (3) preoperative therapy; (4) meticulous surgical technique.
289

\section{Pulmonary Sequestration with Aberrant Systemic Arteries}

R. Capozzi ${ }^{1}$, N. Daddi', S. Mosca ${ }^{2}$, S. Frati ${ }^{2}$,

A.P. Prosperini ${ }^{3}$, F. Ribacchi ${ }^{3}$, C. Fedeli ${ }^{1}$, A. Semeraro',

A. Lomonaco ${ }^{1}$, R. Pecoriello', M. Sciamannini', G. Daddi

${ }^{1}$ S.C. Chirurgia Toracica, Università degli Studi di Perugia,

${ }^{2}$ S.C. Radiologia, Diagnostica e Interventistica, Università

degli Studi di Perugia, ${ }^{3}$ Anatomia Patologica, Azienda

Ospedaliera S. Maria della Misericordia, Perugia

Introduction: Pulmonary sequestration is an uncommon congenital disease in which a portion of lung is supplied by an anomalous systemic artery. We report the case of a woman who had bronchopulmonary sequestration with systemic arterialization of the pulmonary artery.

Methods: A 31 year-old woman (K.J.F.E.) presented in the last three years with fevers, chest pain and productive cough. A chest radiograph on April 2006 revealed an opacity in her left lower lung . After antibiotic treatment the majority of her symptoms improved, chest pain and cough persisted. Admitted in October 2006 to our Unit, she underwent CT scan of the chest that revealed a multiloculated cystic lesion within the left lower lobe. Physical examination showed in- and expiratory crepitant rales at the base of the left lung. Bronchoscopy showed purulent secretions from the left main bronchus; echocardiography as well as spirometry and blood gases revealed normal parameters; angiography showed left lower lobe pulmonary sequestration supplied by an anomalous branch originating from descending thoracic aorta apparently connected to left lower lobe pulmonary artery.

Results: A left lower lobectomy was performed. The surgical specimen injected with coloured solution in the aberrant artery oozed in the pulmonary artery, through a well documented vascular connection, thus confirming the angiographic findings.

Discussion: Pulmonary sequestration with anomalous connection between systemic and pulmonary artery is a very rare entity. Angiography should be the gold standard diagnostic procedure since it provides reliable information on anastomoses between pulmonary and aberrant vessels. Surgical resection is the definitive management.

\section{0}

\section{Surgical Treatment of Pulmonary Metastases: Our Experience}

\section{F. Davoli, A. Bini, J. Brandolini, G. Dolci, F. Sellitri, F. Stella \\ Chirurgia Toracica, Emilia Romagna}

Introduction: The role of surgical resection for pulmonary metastases is evolving; the aim of surgery is mainly radical resection with a curative purpose, then for a diagnostic or palliative intent.

Methods: Between January 2000 and December 2005 a radical matastasectomy was performed in 123 patients $(63 \mathrm{M}, 60 \mathrm{~F}$; the mean age was 63 years). Primary tumour was a carcinoma in 89 cases $(72 \%)$, a sarcoma in $8(6 \%)$, a cutaneous melanoma in $6(4 \%)$. In 50 (40\%) patients the primary tumour was a colorectal cancer, with a Disease Free Interval of 52 months. Among the 123 patients in our 
report were performed 154 pulmonary resections: 106 wedge resections, 40 lobectomies, 5 pneumonectomies and 3 typical segmentectomy. We used the thoracotomic access in the majority of the resections (92\%), VATS was preferred in only 11 cases $(8 \%)$.

Results: Operative mortality was $0 \%$, in the early post-operative period (30 days) was $0.8 \%$ ( 1 of 123 ); mean actuarial survival at 5 -years is $41 \%$ (for metastases from colorectal cancer is $50 \%$; from breast cancer: $25 \%$; from renal cell cancer: $22 \%$ ).

Conclusions: We believe that surgical treatment for pulmonary metastases represents actually the best treatment with good results at long time survival, according to an acceptable risk; multimodal treatment is imperative; in our opinion the most important factor that influences long survival is Disease Free Interval.

\section{1}

\section{Fluoroscopy-Aided Thoracoscopic Surgery (FATS) of Pulmonary Nodules Localized with Platinum Coils}

\author{
A. Droghetti ${ }^{1}$, A. Tagliani ${ }^{2}$, A. Schiavini ${ }^{1}$, U. Rozzanigo ${ }^{2}$, \\ P. Muriana1, C. Bonadiman', R. Caudana², G. Muriana ${ }^{1}$ \\ ${ }^{1}$ Thoracic Surgery Division, ${ }^{1}$ Radiology Division, Carlo \\ Poma Hospital - Mantova, Italy
}

Introduction: Small pulmonary nodules, less than $10 \mathrm{~mm}$ in size, have been increasingly detected with prevalence of computed tomography. A considerable number of lesions resulted to be malignancies. Diagnosis by percutaneous or transbronchial biopsy is difficult, the most recent NICE 2005 guidelines indicate surgical resection as first choice to obtain istology. Inability to use bimanual palpation of the lung to localize nodules during thoracoscopy has necessitated in new methods for detection of small and deep lesions to avoid a thoracotomy. We report our three-year experience of the procedure in which a 'Platinum Coil' is placed around the nodule under pre-operative $\mathrm{CT}$, thus enabling detection and resection of nodules in thoracoscopy by fluoroscopic guidance.

Patients and Methods: Twenty-one patients underwent this procedure for small pulmonary lesions. At the beginning of our experience we marked six nodules with Lipiodol, then we placed fifteen platinum coils under computed tomography guidance (CT).

Results: Between March 2004 and June 2007 all the lesions were successfully identified and resected during fluoroscopy-aided thoracoscopic surgery. The pathologic diagnosis revealed 7 cases of primary lung cancer, metastatic tumors in 4 patients and benign tumors in 10 patients. There were only two cases of pneumothorax which didn't require drainage.

Conclusions: Fluoroscopy-Aided Thoracoscopic Surgery can help the thoracic surgeon to detect small lesions without bimanual palpation and to safely resect them without a thoracotomy.
292

\section{The Surgical Treatment of Pleural Recurrence of Thymoma}

L. Duranti, M. Lucchi, A. Picchi, O. Fanucchi, A. Viti, A. Mussi

Chirurgia Toracica, Pisa

Introduction: We present our overall experience in the surgical treatment of pleural implants in patients who previously underwent resection of a thymoma.

Methods: From January 1980 to June 2006, 20 patients underwent surgical resection of pleural implants, after previous resections for thymoma. The surgical approach at the resection of the thymoma was a VATS in 2, a median sternotomy in 13, a thoracotomy in 2 and sterno-thoracotomy in 3. The initial Masaoka staging was: II A in 2, II $\mathrm{B}$ in 7 and III in 11.

Results: The interval between the thymoma resection and pleural implants surgery ranged from 11 to 156 (mean 60.1) months. Fifteen patients had a thymus related syndrome (13 myasthenia gravis) and 11 improved or remitted after treatment of the implants. All the resection were performed through a postero-lateral thoracotomy. 3 patients underwent a iterative resection for new pleural implants. At the last follow-up 10 patients are still alive (8 diseasefree) and 10 dead (1 for red cell aplasia). Overall 5 and 10-years survival was $43.1 \%$ and $25.8 \%$.

Discussion: Re-operation of thymoma pleural recurrences is safe and achieves satisfactory results in terms of overall survival and paraneoplastic syndrome control. Hypertermic intraoperative pleural chemotherapy and targeted biologic therapies may improve the results of the surgery.

\section{3}

\section{Giant Solitary Fibrous Tumors of the Pleura: The Devil is not as Bad as it seems}

\section{A. Fiorello, P. Laperuta, E. Polimeno, G. Vicidomini, M. Santini}

\section{Thoracic Surgery Unit, Second University of Naples}

Introduction: Solitary fibrous tumors of the pleura (SFTP) represents less than $5 \%$ of pleural neoplasms. They arise from either visceral $(80 \%$ of cases) or parietal pleura and may undergo malignant transformation. We report three cases of giant SFTP undergone surgical resection.

Methods: Case 1: 15 years old man with a mediastinal opacity incidentally discovered at chest radiography. Clinical history and CT features were not suggestive of lymphoma. Case 2: man 65 years old with an enormous space-occupying lesion in the left hemithorax causing left lung collapse and right-side mediastinal shift. Case 3: woman, 47 years old, affected by an opacity in the right chest adherent to diaphragm. In all patients cytological evaluation were not sufficient.

Results: The tumor was removed en bloc through median sternotomy (Case 1) and thoracotomy (Case 2 and 3). Case 1: tumor sizes: $19 \times 14 \times 12 \mathrm{~cm}$, weight: $1,850 \mathrm{~g}$. Case $2: 30 \times 19 \times 18 \mathrm{~cm}$, 4,050 g Case 3: $21 \times 15 \times 13 \mathrm{~cm}, 2,300 \mathrm{~g}$. The histologic diagnosis 
was SFTP (positive for CD 34, and negative for vimentin and cytokeratin), Stage I (Case 1) and Stage 0 (Case 2 and 3) according to De Perrot classification.

Discussion: Surgical resection is the treatment of choice for SFTP. It allows a definitive diagnosis and complete regression of symptoms, with a good clinical outcome. Despite the mass size, the excision is usually simple as the tumor is generally well-circumscribed but must be radical because it is the only guarantee of recurrence and metastasis prevention.

\section{4}

\section{Does Interval Between Perforation and Treatment Influence Prognosis in Patients with Perforation of Thoracic Esophagus?}

\section{A. Fiorello, A. Perrone, F. Napolitano, G. Vicidomini, M. Santini \\ Thoracic Surgery Unit, Second University of Naples}

Introduction: Esophageal perforation is an uncommon clinical entity associated with high mortality rates. Delay diagnosis (more than 24 hours) is generally reported as the most important prognostic factor. We present our experience with patients treated in a delay of more than 36 hours after perforation.

Methods: We performed a retrospective review of 15 patients (10 males and 5 females) with thoracic esophageal perforation. The mean interval between perforation and treatment was 66.8 hours (range: 38 hours to 9 days). Trauma (4 patients), foreign body (4), spontaneous rupture (4), caustic lesion (3) and instrumental lesion (1) caused the perforation in our experience.

Results: The treatment included: primary repair (5 cases), conservative treatment (3), surgical drainage (2), esophagectomy (2), bipolar exclusion (2), Abbot's T-tube drainage (1). Hospital mortality rate was $26.6 \%$ (4/15 patients). The cause of lesion in the group of deceased patients was caustic ingestion in 2, foreign body in 1 , trauma in 1 . Two of these patients had received conservative treatment, 2 esophagectomy. The mean perforation-to-treatment interval was 87.2 hours in the group of deceased patients and 57.4 hours in the group of alive patients. The mean hospital stay was 28.3 days

Discussion: Our experience suggest that esophageal perforation represents a life-threatening clinical entity for which perforation-to-treatment interval still represents an important prognostic factor. However, an accurate drainage of mediastinum and pleural space with rapid lung re-expansion may reduce the additional risk of mortality associated with treatment delay exceeding 24 hours after perforation.
295

\section{Tracheal Injuries after Oro-Tracheal Intubation: Is There a Standard of Care?}

\author{
S. Furia', B. Feil', C. Benato', I. Spilimbergo', \\ G. Biondani ${ }^{1}$, A. Terzi ${ }^{2}$, F. Calabrò ${ }^{3}$ \\ ${ }^{1}$ Scuola di Specializzazione in Chirurgia Toracica Università \\ degli studi Verona; ${ }^{2}$ U.O. Chirurgia Toracica Az. Osp. \\ Cuneo; ${ }^{3}$ U.O. Chirurg ia Toracica Az. Osp. Verona
}

Introduction: Tracheal lacerations after oro-tracheal intubation are a severe and potentially lethal complications of general anesthesia. Surgical repair is considered the treatment of choice, however conservative treatment has emerged as a real possibility in many patients.

Methods: From 1991 to 2006, 11 patients with a median age of 62 years (range 20-85) with iatrogenic tracheal laceration were observed. All patients underwent orotracheal intubation for different elective surgical procedures. Lesions were diagnosed within 12 to 96 hours after injury. Main symptoms were: subcutaneous emphysema, dyspnea, light hemopthoe. Chest CT-scan and fiberoptic bronchoscopy were performed. In all patiens, the injury was in the pars membranacea with a median lenght of $2.5 \mathrm{~cm}$ (range $1.5-5 \mathrm{~cm}$ ). 6 patients were treated conservatively (tear from $1.5 \mathrm{~cm}$ to $5 \mathrm{~cm}$ ), 5 patients (tear $2.5 \mathrm{~cm}$ or more) underwent immediate surgery for the worsening of the clinical coursenot depending on the lenght of the lesion.

Results: Patients treated conservatively were strictly checked clinically, by bronchoscopy (every 2-3 days) and a CT scan before discharge. The mean hospital stay of the entire group was 7.1 days, that of operated patients was 9.4 days.

Conclusion: Nonoperative therapy should be considered in patients with post intubation TBR who are breathing spontaneously, or when extubation is scheduled within 24 hours fr.

\section{6 \\ Surgical Treatment of Multiple Primary Lung Cancers}

L. Libretti, M. Casiraghi, A. Bandiera, A. Carretta, P. Ciriaco, P. Zannini

Chirurgia Toracica, Ospedale San Raffaele, Milano

Introduction: The development of second primary lung carcinomas after surgery for non-small cell lung cancer (NSCLC) is not infrequent. We analyzed the results of surgical treatment of multiple synchronous or metachronous lung carcinomas (MPLC).

Methods: From January 1993 to June 2007, 57 patients underwent surgery for MPLC. Twelve patients had a synchronous and 45 a metachronous tumor, 10 of these patients subsequentely developed a third tumor. In patients with metachronous tumors the mean interval between the first two operations was $(53.7 \pm 43.37)$ months. A total number of 122 operations was performed ( 2 pneumonectomy, 8 completion pneumonectomies, 61 lobectomies, 3 sleeve lobectomies, 4 bilobectomies, 3 segmentectomies and 41 wedge resections). Histology showed 77 adenocarcinomas, 35 squamous carcinomas and 
12 other NSCLC types. Postoperative tumor stage was I in 112 cases, II in 9 and IIIa in 3.

Results: Two patients died postoperatively (3.5\%). Overall 5and 10 -year survival was $86.8 \%$ and $76.7 \%$ respectively. In patients with metachronous tumors 5- and 10-year survival from the time of the first diagnosis was $90.2 \%$ and $79.4 \%$ respectively. Survival after the development of a metachronous lesion was $61.4 \%$ at five years. Three-year survival for patients with synchronous tumors was $74 \%$.

Discussion: The possibility of a second primary lung cancer after curative resection for NSCLC should always be considered and long-term follow-up after surgery is mandatory. Surgery enables favorable results to be achieved in the treatment of MPLC.

\section{7}

\section{Solitary Fibrous Tumors of the Pleura: Immunohistochemical Analysis and Evaluation of Prognostic Factors after Surgical Treatment}

\author{
A. Bandiera, L. Libretti, M. Casiraghi, M. Giovanardi, \\ L. Ferla, A. Carretta, P. Zannini \\ Unità di Chirurgia Toracica, Ospedale San Raffaele, Milano
}

Objective Solitary fibrous tumors of the pleura (SFTP) are rare neoplasms with unusual histological and clinical features. Although surgery is the treatment of choice for SFTP, tumor recurrence may occur after complete resection. The aim of the study was to identify the clinical and pathological features of SFTP that are associated with a higher risk of recurrence after surgical treatment. Methods From May 1995 to May 2007 twenty-five patients (15 female, 10 male; mean age 60 years) with SFTP underwent surgical treatment at our Department. Mean follow-up was 69 months. Mean tumor size was $10 \mathrm{~cm}$. The tumors were peduncolated in 15 patients, sessile in 7 and inverted in 3. Histological features were benign in 22 patients and malignant in 3. Results Five-year and ten-year disease-free survival were $94 \%$ and $77 \%$, respectively. A worse disease-free survival was observed when SFTP were sessile, originated from the parietal pleura, had malignant histological features and a lower expression of progesterone receptors $(p<0.05)$. Symptomatic patients at the time of the diagnosis also had a higher incidence of tumor recurrence $(p<0.05)$. Conclusions Clinical and pathological characteristics such as malignant histology, sessile morphology and an origin from parietal pleura identify SFTP with a higher risk of recurrence after surgery, and which thus require strict follow-up.

\section{8}

\section{Results in Trimodality Treatment for MPM}

S. Lovadina, F. Lo Giudice, A Ferronato, R. Salvi, P. Fontana, A. Zaccaria, V. Pagan

Chirurgia Toracica, Mestre

Introduction: The promising results of Sugarbaker's trimodality treatment for malignant pleural mesothelioma (MPM) are debated and not yet uniformly replicated. The purpose of this study is to evaluate prospectively the reproducibility of the treatment results in a patient population with mesothelioma staged by the IMIG classification.

Methods: 54 patients with MPM have been judged candidable to extended pleuropneumonectomy (EPP), to be followed by chemotherapy (paclitaxel + carboplatin) and radiotherapy (50 Gy).

Results: At thoracotomy, 44 of the 54 patients (81\%) underwent EPP; $73 \%$ of the operated completed the entire adjuvant chemo-radiotherapy with no major toxicity. The 30-day or in-hospital operative mortality rate was $2(4.5 \%)$, the major morbidity $36 \%$, and the overall complication rate $50 \%$. At 5 years the projected survival of the 42 surgical survivors submitted to EPP is $19 \%$; median survival is 20 months. The restricted group of patients with epithelial, N0-1, completely resected MPM (R0) exhibits a projected 50\% 5-year survival. Clinical understaging has shown up to be noticeable both at the surgical exploration and pathology examination. Most of the disease recurrences are loco-regional and the current insufficiency of intraoperative or postsurgical radicality needs improvement, along with earlier diagnosis, more accurate staging.

Discussion: This series confirms the reproducibility of the trimodality treatment for MPM, which is associated with prolonged survival for early-stage tumors at the cost of a not prohibitive treatment-related mortality rate.

\section{9 \\ Staging and Treatment of TOS \\ F. Lo Giudice, S. Lovadina, A. Ferronato, R. Salvi, P. Fontana, A, Zaccaria, V. Pagan}

Chirurgia Toracica, Mestre

Introduction: Thoracic Outlet Syndrome (TOS) is a well known syndrome. Sophisticated imaging techniques can clearly highlight any anatomical damage and many therapeutic choices are available. It would seem obvious that any given patient should obtain the same treatment irrespective of the medical institution he contacts. Instead each specialist may recommend different treatments: physiatrist, neurologist, surgeons (thoracic, vascular, neuro, orthopedic). There is no univocal treatment plan consensus for this pathology. Correct staging of TOS is still an unresolved question.

Methods: To solve this problem, we collected all clinical and instrumental aspects of the syndrome into a clear classification. Similar to TNM staging of tumors, we used a grouping model based on anatomical structures: $\mathrm{N}$ (= Nerves; brachial plexus and sympathetic fibers), A (= Artery; subclavian-axillary), V (= Vein; s-a) named NAV. From 1984 to 2006 we evaluated 188 patients with TOS. These were grouped in 4 stages depending on their NAV status. Therapy was in accordance with stage.

Results: $38 / 188$ patients $(20 \%)$ underwent surgical approach. The outcome was excellent to fair in 29 patients $(76 \%)$, worse or persistent in $9(24 \%)$ but the subjective improvement was only $50 \%$. Examination of our records confirmed a valid and useful correlation between the proposed NAV staging and the therapeutic procedures that were actually applied.

Discussion: On the basis of our experience, the NAV staging system is useful for correct patient grouping. Now a prospective multicentre study is needed for universal scientific validation. 
300

Sleeve Lobectomy at Monaldi Hospital: 30-Years Experience in a Single Institution

P. Maietta, V. de Matteis, A. Maiorino, M.R. Massimo

Cattedra Di Chirurgia Toracica II Universita' Di Napoli-Aorn Monaldi

Introduction: The sleeve lobectomy is thoracic surgical procedure for central tumors as a alternative to pneumonectomy. The objective of this study is to evaluate long-term results of sleeve lobectomies for NSCLC.

Methods: A retrospective study of 120 patients who underwent sleeve lobectomy for NSCLC between 1977-2007 was undertaken. There were $90(75 \%)$ men and $30(25 \%)$ women with a mean age of 62.3 years $(24-86)$. 48 patients $(40 \%)$ had a preoperative contraindication to pneumonectomy. Right upper lobectomy was the most common operation (49.4\%). Vascular sleeve resection was perfomed in 10 patients $(8.3 \%)$. The histologic type was predomintly squamous cell carcinoma 90 (75\%) and adecarcinoma (20\%).

Results: There was no operative deaths; the operative morbidity rates was $25 \%$. Follow-up was complete in 110 patients (91\%). overall 5 year and 10 year were $53 \%$ and $28.6 \%$ respectively.

Discussion: According to the literature, sleeve lobectomies give local tumor control, even in patients with preoperative controindication to pneumonectomy. Postoperative complicatications are higher in smokers, $\mathrm{N}$ disease, right resections, bilobectomies.

\section{1 \\ Experimental Evaluation of a New System for Lung Aerostasis \\ G. Marulli, M. Schiavon, P. Villoresi, S. Bonora, S. Roman, F. Calabrese, C. Giacometti, F. Lunardi, F. Rea \\ Chirurgia Toracica, Padova}

Introduction: Persistent alveolar air leaks after pulmonary resection contribute significantly to increased patient morbidity and prolonged hospitalization. This research study was designed to investigate the sealing capacity of an experimental kit for lung aerostasis.

Methods: The kit is composed by a semiconductor laser system applied on a protein substrate associated with a chromophore which increases absorption. In vitro tests on porcine lung tissue were done to define ideal laser parameters (power $100 \mathrm{~A}$, frequency $50 \mathrm{~Hz}$ and pulse duration $400 \mu \mathrm{s}$ ) and protein substrate dilution (50\%). For in vivo tests 14 pigs through a left thoracotomy received two different lung damages: a linear incision and a circular incision mimicking enucleating resection. Protein substrate applied on damaged areas was treated with laser to obtain a layer that reconstituted the integrity of visceral pleura. Air leaks were intraoperatively evaluated by water submersion test. Animals were sacrificed at postoperative day 0 and 7 to study early and late pathological features.

Results: After applying laser treatment no air leaks were seen in all proofs except in two cases in which a second application was required to seal small peripheral air leaks. At time 0 pathological damage was mostly constituted by superficial necrotic tissue covered by protein membrane. At time 7 a complete recovery of lesions by fibrous scar without inflammation of adjacent lung tissue was seen.

Conclusions: This experimental study demonstrated the effectiveness of this new kit for sealing air leaks after lung surgery. Further studies need to verify human application.

\section{2 \\ Partial or Total Sternectomy for Neoplastic Disease: Different Reconstructive Techniques in our Decennal Experience}

\author{
S. Sanna, S. Folli, M. Mengozzi, M. Taurchini, \\ M. Monteverde, D. Argnani, D. Dell'Amore \\ U.O. Chirurgia Toracica, Forlì
}

Introdution: Malignant sternal tumours are uncommon. A radical resection can often provide a complete cure. For sternal reconstruction various protheses reinforced and covered with soft tissue muscular or musculo-cutaneous flap are used. We present our experience in the treatment of sternal tumours.

Methods: Between 1995 and 2007 we treated 11 patients, 6 men and 5 females affected by sternal tumours. 7 patients presented a primary tumour, 4 metastatic disease. 8 cases underwent subtotal, 3 total sternectomy. For sternal reconstruction we used Marlex protheses, with methylmethacrilate in 6 cases, 2 silicon tubes in 2 and 2 metallic plates in 1.10 patients had a muscular flap coverage, with pectoralis maior (7) or latissimus (3), one patient a musculo-cutaneous flap.

Results: No post-operative mortality or major complications occured. Minor complications were an infection of the protheses treated with toilette and omental flap; a pleural effusion resolved with evacuative thoracentesis. The mean time of drainage and hospital stay were 4.5 and 5.6 days.

Discussion: Surgical resection and reconstruction represent a basic step in the treatment of all sternal tumours. The combination of prosthetic materials, necessary to mantain ventilatory mechanics and to protect intrathoracic organs with muscular or musculo-cutaneous flaps are advantageous permitting a well vascularize sternal reconstruction.

303

Tracheal Reconstruction with Sternocleidomastoid Myoperiosteal Flap for Post-Thyroidectomy Tracheal Necrosis: A Case Report
S. Sanna, M. Mengozzi, M. Taurchini, M. Monteverde, D. Argnani, D. Dell'Amore
U.O. Chirurgia Toracica, Forlì

Tracheal necrosis after thyroidectomy is a rare complication but lethal and difficult to treat. The best treatments are tracheal resectionanastomosis and myoplastic flap coverage. We present a case of postthyroidectomy tracheal necrosis successfully treated with myoplastic flap. 
Case Report: We observed a young african girl undergone extensive thyroidectomy and cervical node dissection for papillar carcinoma with a suspected trachal lesion. Bronchoscopy showed a $5-\mathrm{cm}$ loss of the right antero-lateral tracheal wall. Recervicotomy showed an important wall necrosis avoiding tracheal resection or tracheostomy. We performed a myoplastic flap with bilateral sternocleidomastoid and pre-thyroid muscles. The 7 post-op day bronchoscopic control showed the repair of tracheal loss and minimal functional stenosis. The patient was discharged to the hospital in 12 post-op day.

Discussion: The endocrine surgeon performing thyroidectomy must often project oncologic tracheal resection. The reconstructive tracheal techniques are various from direct tracheal suture to laryngotracheal dissociation. The best treatment is segmental resection with end-to-end anastomosis. If not technically possible myoplastic flap reconstruction with latissimus dorsi sternocleidomastoid periosteal and pre-thyroid muscles is useful. This procedure permits normal phonation and good functional and oncologic results.

\section{4 \\ Our Experience in Surgical Treatment of Catamenial Pneumothorax}

G.L. Pariscenti, M. Benvenuti, D. Benetti, G. Botticini, T. Ligabue, P. Bovolato

Thoracic Surgery Department, Civil Hospital, Brescia - Italy

Introduction: Catamenial pneumothorax $(\mathrm{CP})$ is a spontaneous pneumothorax which occurs within 72 hours before or after menstruation. The aetiology is still controversial. Very few cases have been reported in literature.

Methods: Description of clinical course and surgical treatment of 8 women with CP.

Results: Eight women (mean age 34.5; range 28-41) had spontaneous pneumothorax associated with menses. In 6 cases an associated abdominal endometriosis was known and 1 patient had had tubal/ovarian resection. All CP were right-sided and we found multiple small diaphragm perforations with adjacent endometrial tissue and pleural bleeding implants. Treatment were as follow: 1 VATS with talc pleurodesis, 1 thoracotomy for hemo-pneumothorax with direct suture of the diaphragm defects, 6 video-assisted minithoracotomy with diaphragm covered with mesh followed by mechanical or talc pleurodesis. In all patients coagulation of the implants was performed. Surgical treatment was followed by a course of gonadotrophin-releasing hormone analogue therapy in 6 patients: no recurrence was noted. Partial hemothorax was reported in 1 woman treated with mesh who refused hormonal therapy.

Discussion: CP is under diagnosed and should be suspected in ovulating women with spontaneous pneumothorax. During VATS procedure, inspection of the diaphragm is mandatory. In our experience, best results are obtained when surgical treatment (video-assisted minithoracotomy with diaphragm lesions covered with mesh) is associated with hormonal therapy.
305

Primary Intra-Extravascular Leiomyosarcoma

of the Superior Vena Cava: Surgical

Resection with Jugular-Femoral Extracorporeal Circulation

\author{
G.L. Pariscenti, D. Benetti, M. Benvenuti, G. Botticini, \\ T. Ligabue, P. Bovolato \\ Thoracic Surgery Department, Civil Hospital, Brescia - Italy
}

Introduction: Primary leiomyosarcoma arising from superior vena cava (SVC) is exceptional: only 12 cases are reported in literature. We describe the case report of surgical resection and jugularfemoral extracorporeal circulation during SVC temporary clamping.

Methods: A diabetic, hypertensive 65-year old woman arrived at Emergency with dyspnoea, cough, dysphonia, and important SVC syndrome. Contrast-enhanced computed tomography demonstrated a mass in the right paratracheal region with intraluminal filling defect in SVC confirmed with conventional phlebography with inverted flow into a dilated azygos vein. A right axillary minithoracotomy for biopsy was performed. One week later surgical resection was performed in median sternotomy. An extracorporeal circulation with single roller pump between right interior jugular vein and femoral vein was started before temporary clamping of the SVC. Reconstruction was made with bovine pericardium tubular graft between SVC at origin of azygos vein and right auricula. No complication was reported.

Results: Leiomyosarcoma arising from SVC, free margins of resections and no nodal involvement was diagnosed. The woman is alive and disease free at 10 months.

Discussion: Radical resection gives good results in terms of survival and quality of life. The jugular-femoral extracorporeal circulation avoid risks deriving from the clamping of SVC. The risk full controlled hypertension is not necessary and clamping time can be prolonged. It is a safe procedure that can be used also in resection of other tumours involving SVC.

306
Uterine Leiomyoma Metastatic to the Lung
R. Pecoriello, A. Semeraro, C. Fedeli, M. Ragusa,
M. Sciamannini, N. Daddi, A. Lomonaco, R. Capozzi,
G. Daddi
S.C. Chirurgia Toracica, Università degli Studi di Perugia

Introduction: Uterine leiomyomas belong to a heterogeneous family of tumors with peculiar biological behaviour. Pulmonary metastases may originate from frankly malignant leiomyomas (sarcomas) as well as from so called benign metastasizing leiomyomas. Latency between hysterectomy and metastatic disease may vary from few months to several years. We report two cases with an exceptionally long latent period.

Methods: Two cases of apparently primary giant lung tumor were observed. Both presenting clinical history of fever and malaise. Chest x-ray showed in both cases a left pulmonary opacity. Preoperative work-up yielded the diagnosis respectively of epithelial 
and mesenchymal tumor without extrapulmonary localization (CT and PET-CT).

Results: Case N 1: 68 year-old (Z.A.), the giant lobulated tumor, invading the hilar structures, required a left pneumonectomy. Histology revealed a metastatic uterine leiomyoma. She had undergone hysterectomy for multiple leiomyomas 21 years previously. Case N 2: 66 year-old (M.M), was submitted to left pneumonectomy for a giant tumor extended to both lobes. Also in this case metastasis from uterine leiomyoma was diagnosed and confirmed by comparison with histology from hysterectomy (performed 16 years previously). There was no peri-operative mortality. Both the patients are alive without evidence of disease 6 and 3 months after surgery.

Discussion: These cases suggest that the diagnosis of primary pulmonary sarcoma needs careful pathological assessment and follow-up of the patients submitted to hysterectomy for leiomyoma should be protracted.

\section{7}

Surgical Treatment of Neuroendocrine Tumors of the Lung: Outcome and Results in a Series of 129 Patients

\author{
A. De Palma' , D. Loizzi², R. Quercia' , F. Tota' , F. Sollitto², \\ M. Loizzi ${ }^{1}$ \\ ${ }^{1}$ Cattedra di Chirurgia Toracica, Università degli Studi di \\ Bari, ${ }^{2}$ Cattedra di Chirurgia Toracica, Università degli Studi \\ di Foggia
}

Introduction: The objective of this study was to evaluate prognostic factors and long-term survival in patients surgically treated for neuroendocrine lung tumours at our Institutions.

Methods: From January 1987 to August 2007, 129 patients (79 men, 50 women), mean age 56.7 years (range: 16-78 years) were submitted to lung resection for neuroendocrine tumours (NET). All cases were rewieved according to WHO/IASLC classification (1999) and divided in tumorlets, typical carcinoid (TC), atypical carcinoid (AC), large cell neuroendocrine carcinoma (LCNEC) and small cell lung cancer (SCLC).

Results: We performed 17 pneumonectomies, 13 bilobectomies, 2 sleeve-lobectomies, 64 lobectomies, 2 segmentectomies, 30 wedge resections, 1 bronchotomy. Pathological examination revealed: 7 tumorlets, 54 TC, 24 AC, 14 LCNEC, 30 SCLC. Mean follow-up was 61 months (range: 0-240 months). 5-year survival was $92 \%$ for TC, $71 \%$ for AC, $20 \%$ for LCNEC, $23 \%$ for SCLC (p < 0.001 ); $85 \%$ for TC-AC-LCNEC N0, 49\% for TC-AC-LCNEC N1-N2 (p < 0.001), $56 \%$ for SCLC N0, $6 \%$ for SCLC N1-N2 (p < 0.05 ).

Discussion: In our experience, lymph node status associated to histological type was the most important prognostic factor in patients submitted to lung resection for neuroendocrine lung tumours: therefore, systematic mediastinal lymphadenectomy should be accomplished in all histological subtypes.

\section{8 \\ Postoperative Outcome after Neoadjuvant Therapy for NSCLC}

\author{
C. Rapicetta, L. Luzzi, L. Voltolini, F. Granato, S. Tenconi, \\ C. Ghiribelli, G. Gotti \\ UOC Chirurgia Toracica, Siena
}

Although the benefit of neoadjuvant therapy in long-term survival of patients with NSCLC is well accepted, its impact on postoperative mortality and morbidity is not yet established.

Materials and Methods: We reviewed records of 83 consecutive patients who underwent thoracotomy after induction therapy between 1996 and 2006 (Group 1). Results were compared to those of a control Group of 166 patients surgically treated in the same decade without prior neoadjuvant therapy (Group 2).

Results: The two groups were homogeneous for age, sex, histology, comorbidity and respiratory function. There was no hospital mortality. Cumulative incidence of major complications was 32 in Group 1 and $37 \%$ in Group $2(\mathrm{p}=0.18)$. The incidence of each complication considered did not significantly differ between the two Groups. A higher percentage of patients in Group 1 required blood transfusions $(22 \%$ vs. $4 \%-\mathrm{p}<0.0001)$. Multivariate analysis showed Forced Expiratory Volume in 1 second (FEV1) $<75 \%$ of predicted $(p=0.018)$ and blood transfusions $(p=0.006)$ to be independent risk factors for major postoperative events.

Conclusions: Preoperative chemotherapy did not affect morbidity and mortality. Patients with a FEV $1<75 \%$ of predicted or requiring blood transfusions seem to be at increased risk of developing major complications.

\section{9 \\ Double Large, Unusually Located Pericardial Cyst}

F. Raveglia, A. Baisi, A.M. Calati, S. Meda, A. Leporati

Chirurgia Toracica, Milano

Pericardial cysts are mesothelial congenital lesions classically located in the anterior cardiophrenic angle. Different locations are rare.

A 82 years-old man was affected by a cyst in the upper mediastinum of $7 \times 6 \times 4 \mathrm{~cm}$. CT scan after 12 months showed meaningful enlargement of the cyst up to $10 \times 9 \times 8 \mathrm{~cm}$ compressing the Superior Vena Cava.

Surgical treatment was proposed because of the uncertain diagnosis and the compression on contiguous organs.

After the introduction of two trocars, a muscle sparing right thoracotomy was performed due to stucking pleural adhesions. A right paratracheal thin-walled cystic lesion compressing both the superior vena cava and the azygos vein was found. Upon completed dissection of the lesion wall, the cyst was removed. A second smaller cyst, not diagnosed at the CT scan, was found behind the first one in the inner part of the mediastinum. It was also removed. The histological examination diagnosed a double pericardial cyst.

Rarely pericardial cysts are located outside of the right or left cardiophrenic angle. Stoller in his review form 1929 to 1985 mentioned 
only 34 cases. From 1985 to now few series of atypically located cysts have been reported. Bilocular or double pericardial cyst are never reported.

Surgical resection is widely stated in symptomatic cases and when the diagnosis remains uncertain. Asymptomatic cysts should be considered for surgery in case of potential risk of compression on contiguous structures or when CT shows progressive enlargement. When feasible thoracoscopy is the technique of choice.

\section{0}

\section{Preoperative CT-Guided Localization of Pulmonary Nodules with a Hookwire before Video-Assisted Thoracospic Surgery}

M. Riccardo', L. Cancian², M. Pavanello', A. Piazza1, G. Morana², G. Di Falco

${ }^{1}$ Department of Surgery I, ${ }^{2}$ Department of Diagnostic and Interventional Radiology, Treviso Regional Hospital

Introduction: Video-assisted thoracic surgery (VATS) is a routinely used in pulmonary surgery. Small pulmonary nodules represent the most common reason for VATS. The purpose of our study was to evaluate the effectiveness of hookwire CT-guided localization of peripheral pulmonary lesions.

Methods: From 1996 to 2006, percutaneous localizations with CT guidance of 85 lesions, who underwent VATS resections for small pulmonary nodular lesions, was performed in 85 patients. Patients were classified into two groups: 34 pts treated from 1996 to 2002 and 51 pts from 2003 to 2006.

Results: The hookwire was successfully placed without dislodgment in $71 / 85$ lesions $(83 \%)$. The success rate in the first group was $25 / 34(73 \%)$, in the second group $46 / 51(90 \%)$. In 85 placements, nonsymptomatic pneumothorax cases was observed in 10 pts $(12 \%)$, hemorragies into the lung were observed in $6 \mathrm{pts}(7 \%)$. Thirteen pts $(38 \%)$ in the first group and 14 pts $(27 \%)$ in the second group required conversion to a standard thoracotomy. The most common reason was hookwire dislocation in 6 pts $(18 \%)$ in first group and in 4 pts $(8 \%)$ in second group.

Discussion: Preoperative CT guided hookwire localization before VATS is an effective technique. For good results is essential the radiologist experience and the collaboration with surgeons.

\section{1}

\section{Radio-Frequency Tumor Ablation (RFA) in Primary Inoperable Lung Cancer}

\author{
M. Riccardo 1 , L. Cancian², P. Lucernoni3 , U. Sannini, \\ G. Morana², G. Di Falco \\ ${ }^{1}$ Department of Surgery I, ${ }^{2}$ Department of Diagnostic and \\ Interventional Radiology, ${ }^{3}$ Department of Pneumology, \\ Treviso Regional Hospital
}

Introduction: The purpose of our study was to report our initial experience and to evaluate the feasibility, safety, and initial therapeutic effect of RFA ablation in the treatment of unresectable lung cancer.

Methods: From June 2005 we treated with RFA 8 pts with primary non-small cell lung cancer, nonsurgical candidates because of medical comorbidities. Pts were treated with radiofrequency ablation after agreement among oncologists, thoracic surgeons, and interventional radiologists. CT guidance and general anesthetic were used for all pts. RF energy was applied with LeVeen Needle. The results was evaluated with first CT scan at 30 days, follow-up was from 2 to 24 months.

Results: RF ablation was technically successful in all lesions with almost complete necrosis. No periprocedural mortality occurred. Necrosis of tumor was greater than $90 \%$ in all lesions based on shortterm follow-up imaging. Complicances was: 8 pneumothoraces $(100 \%)$, pleural effusion in 6 pts $(75 \%)$ and transitory respiratory insufficiency in 2 pts $(25 \%)$. One patient died for cardiac failure. Two patients are in disease progression at 18 months follow-up.

Discussion: Lung RF ablation is a feasible, relatively safe, and promising treatment for unresectable lung tumors. A multidisciplinary approach offers valuable expertise for patient care. The association with radiation-therapy must be evaluated.

\section{2 \\ The Role of Gastroesophageal Reflux Disease in Lung Transplant

\author{
G. Rizzardi, L. Zanatta, C. Giacometti, G. Marulli, \\ C. Rizzetto, F. Lunardi, M. Loy, F. Rea \\ Chirurgia Toracica, Università degli Studi di Padova
}

Background: Long-term survival in lung transplantation is limited by Bronchiolitis Obliterans (BO), the pathological setting of chronic rejection. Recent studies indicated gastroesophageal reflux (GERD) as a possible cause of allograft dysfunction. Our aim is to analyze the prevalence of GERD in transplant and study an index that could identify patients with microaspiration and then at risk of BO.

Methods: From December 2005 to June 2007, 16 lung transplanted patients were evaluated. Our protocol included spirometry, bronchoscopy with transbronchial biopsies and bronchoalveolar lavage fluid (BALF) collection, lipid-laden macrophage index (LLMI) evaluation to determine the alveolar macrophage cytoplasm lipid content, upper endoscopy, gastric emptying study, esophageal manometry and 24-hour pH-monitoring.

Results: There were 14 male and 2 female; median age was 49.5 years (range 28-65). We observed the patients with a median time of 5 months (range, 2-84) after transplantation. GERD had a prevalence of $56.3 \%$ (9/16 patients), documented at $\mathrm{pH}$-monitoring. Gastric emptying was prolonged in $75 \%$ of patients. Esophageal manometry revealed an abnormal esophageal motility in $1 / 3$ of patients; endoscopy evidenced the presence of esophagitis in 5 patients. LLMI was suggestive of aspiration (abnormal value $>85$ ) in $5 / 8(62.5 \%$ ) patients with GERD and in 3/7 (42.8\%) patients without GERD, in one case it was not possible to perform BALF collection.

Conclusions: we confirmed an increased prevalence of GERD and prolonged gastric emptying in lung transplant patients. Increased LLMI tends to be associated with GERD, possibly reflecting microaspiration. It may be used to select patients will benefit from more aggressive treatment (fundoplication). 


\section{3}

\section{Sleeve Lobectomy for Non-Small Cell Lung Cancer: Our Experience Overtime}

M. Schiavon, G. Marulli, A. Zuin, S. Nicotra, G. Rizzardi, M. Bulf, A. Sartori, F. Rea

Divisione chirurgia toracica, Padova

Introduction: To date sleeve lobectomy is an effective and safe procedure for NSCLC in alternative to pneumonectomy even in patient with good pulmonary function. We sought to analyze our experience in two consecutive period (1980-1995 vs. 1996-2006) to compare the evolution of surgical strategy and the factors influencing outcome and long-term survival.

Methods: From 1980 to 2006, 217 patients underwent sleeve procedures, 84 and 133 in the periods 1980-1995 and 1996-2006 respectively. In 44 patients $(20.3 \%)$ a vascular procedure was associated. Thirty patients $(13.6 \%)$ had preoperative irradiation and twentyeight $(12.7 \%)$ had preoperative chemotherapy.

Results: Overall 5-years survival rate was $37.9 \%$ and stage by stage analysis revealed a significant survival difference between stage I-II and III $(p<0.01)$. Overall postoperative morbidity was $16.1 \%$ and mortality was $4.1 \%$. Preoperative radiotherapy was identified as significant risk factor for early bronchial complications $(p=0.02)$ and perioperative mortality $(\mathrm{p}=0.007)$. In second period we use less frequently preoperative radiotherapy $(\mathrm{p}=0.003)$ and more frequently induction chemotherapy $(\mathrm{p}=0.0003)$ and an associated vascular procedure $(\mathrm{p}=0.01)$. No differences in survival, early and late morbidity were observed, but mortality was significantly higher in the first period $(9.5 \%$ vs. $0.7 \%, \mathrm{p}=0.02)$.

\section{4}

\section{Brain Metastases in Patients with Lung Cancer: Results of Surgical Treatment}

\section{A. De Palma1, D. Loizzi², E. Spada', R. Quercia', G. Garofalo' ${ }^{1}$, M. Loizzi ${ }^{1}$ \\ ${ }^{1}$ Cattedra di Chirurgia Toracica, Università degli Studi di Bari, ${ }^{2}$ Cattedra di Chirurgia Toracica, Università degli Studi di Foggia}

Introduction: The objective of this study was to analyze patients with primary lung cancer and synchronous or metachronous brain metastases and compare survival of those submitted to both pulmonary tumour and brain metastases resection with survival of those who underwent a single intervention and/or medical treatment only.

Methods: We analysed data of 27 patients ( 23 men, 4 women), with a mean age of 60.8 years: 11 patients (group A) underwent both lung and cerebral resection, 16 patients (group B) were submitted only to lung (4) or brain (4) intervention or to neither of them (8). All patients received chemotherapy.

Results: Pathological examination revealed: 12 adenocarcinoma, 11 squamous carcinoma, 2 neuroendocrine tumor, 2 undifferentiated carcinoma. One-year survival from the diagnosis of the disease was $91 \%$ and $47 \%$, 2-year survival $61 \%$ and $20 \%$, 3-year survival $51 \%$ and $0 \%$, 5 -year survival $19 \%$ and $0 \%$ in group A and B respectively $(\mathrm{p}<0.05)$.
Discussion: In group A patients surgically treated with both lung and brain metastases resection obtained a better survival compared to patients of group B, who received a single intervention and/or medical treatment only. All of group B patients died within three years from the diagnosis of the disease. Whenever possible, it is advisable to submit patients to an early surgical resection of both primary lung tumour and brain metastases.

\section{5 \\ Awake VATS in the Treatment of Spontaneous Pneumothorax}

F. Tacconi, D. Forcella, E. Pompeo, T.C. Mineo

Cattedra di Chirurgia Toracica-Policlinico Università Tor Vergata-Roma, Direttore.: Prof. T.C. Mineo

Introduction: We assessed in a prospective study the feasibility and efficacy of awake VATS bullectomy-pleural abrasion to treat spontaneous pneumothorax.

Methods: Between January 2001 and June 2005, 21 patients with spontaneous pneumothorax underwent awake VATS bullectomypleuroabrasion under sole thoracic epidural anesthesia and spontaneous ventilation. Primary outcome measures included technical feasibility, as scored into 3 grades (from 1, unsatisfactory, to 3, excellent) and recurrences within 12 months. Secondary outcome measures included global operating room time, perioperative pain control, and postoperative hospital stay.

Results: Technical feasibility was scored as excellent, good, and satisfactory in 8,7, and 6 patients, respectively. No patient required conversion to general anesthesia. Global operating room time was $78.0 \pm$ 20.0 minutes. Postoperative visual analog pain scale score was $2.0 \pm 3.0$. Postoperative hospital stay was $2.0 \pm 1.0$ days, without significant morbidity. A total of 5 patients $(23.8 \%$ ) could be discharged within the first 24 postoperative hours. Overall procedure-related cost showed significantly lower if compared with median cost of VATS bullectomy-pleuroabrasion under general anesthesia at our institution (2,540 euros \pm 352 euros vs. 3,550 euros \pm 435 euros, $\mathrm{p}<0.0001$ ). One patient only had recurrence occurring within 12 months after surgery.

Discussion: In our study, awake VATS bullectomy with pleural abrasion proved feasible and resulted in short hospital stay and reduced procedure-related costs, while providing equivalent outcome to the usual procedure.

\section{6 \\ Pleurectomy and Intrapleural Hypertermic Chemotherapy for Malignant Pleural Mesothelioma: Our Experience}

S. Tenconi, F. Granato, M. Gallazzi, C. Ghiribelli, L. Luzzi, P. Paladini, L. Voltolini, G. Gotti

Chirurgia Toracica, AOU Senese, Siena

Introduction: This study reviewed our experience with a sperimental protocol of pleurectomy and decortication (PD) followed by 
intrapleural hypertermic chemotherapy (IHC) in patients not eligible for extrapleural pneumonectomy (EPP).

Patients and Methods: Between January 2006 and June 2007 10 patients were enrolled in the study. According to literature, none of these patients were eligible for EPP. All patients had at least one risk factor for poor prognosis according to CALGB classification. Nevertheless, all patients had a good ASA score and resectable disease at preoperative assessment. Diagnosis was obtained by VATS biopsy and all patients underwent PD followed by $100 \mathrm{mg} / \mathrm{mq}$ Cisplatin intrathoracic perfusion at $41{ }^{\circ} \mathrm{C}$ for 50 minutes. Cisplatin and Alimta adjuvant chemotherapy was administered in 9 cases.

Results: No perioperative mortality was observed and postoperative complications occurred in 3 cases. 3 patients had macroscopic residual disease, 1 had microscopic infiltration of resection margins. Median survival was 10 months, 3 patients died of progression of disease within 12 months. No cisplatin related toxicity was observed. Among surviving patients nobody has relapsed so far for pleural effusion, chest pain or contamination of surgical wounds.

Discussion: Despite a very short follow up, we can argue that PD with IHC has to be considered a safe procedure to be executed in more compromised patients affected by resectable MPM. Perhaps intrapleural use of hypertermic cisplatinum should prevent neoplastic cell dissemination, reducing chest involvement and thus improving actual quality of life, but not survival of patients.

\section{7 \\ Does Postoperative Albumin Administration Improve Outcome in General Thoracic Surgery?}
A. De Palma', F. Tota', N.P. Ardò', E. Spada', O. Pizzuto', D. Loizzi
${ }^{1}$ Cattedra di Chirurgia Toracica, Università degli Studi di Bari, ${ }^{2}$ Cattedra di Chirurgia Toracica, Università degli
Studi di Foggia

Introduction: The objective of this study was to assess if albumin administration in patients submitted to lung resection significantly improves clinical outcome, reducing hospital stay and costs.

Methods: We analysed data of 36 patients who underwent lung resections (12 lobectomies, 1 bilobectomy, 1 segmentectomy, 22 wedge resections) for various diseases at our Institution from April 2006 to August 2007. Exclusion criteria from our study were: age $<50$ years, diabetes, nephropathies, hepatopathies, systemic vasculopathies, previous chemo-radiotherapy. Patients were distinguished in 2 groups, homogeneous for age, surgical access, type of lung resection and postoperative albumin value, one submitted to albumin administration (group 1) and one which didn't received albumin treatment (group 2).

Results: Mean chest tube duration was $6.8 \pm 1.1$ and $7.7 \pm 1.8$ days $(\mathrm{p}=0.041)$ in group 1 and 2 , respectively; mean postoperative hospital stay was $8.7 \pm 1.8$ e $8.8 \pm 1.9$ days $(\mathrm{p}=0.430)$ in group 1 and 2 , respectively.

Discussion: In group 1 albumin administration after lung resection surgery was associated with shorter chest tube duration and postoperative hospitalization, compared to group 2; however difference was statistically significant for chest tube duration only. Postoperative albumin administration in patients submitted to lung resection could be routinely used to improve clinical results.

318

\section{Sentinel Lymph Node Mapping (SLN): Detection of Micrometastases by Polymerase Chain Reaction in Stage I NSCLC}

\author{
A. Viti, F.M.A. Melfi, A. Picchi, O. Fanucchi, A. Mussi \\ Chirurgia Toracica, Pisa
}

Objective: We previously reported the results achieved in detecting SLN. We applied molecular techniques (RT-PCR) to improve the detection of micrometastasis to evaluate an improvement of staging in early NSCLC patients (pts).

Methods: Since January 2005 we applied the SLN-mapping in 22 Stage I-NSCLC pts. A dose of $37 \mathrm{MBq}\left(1 \mathrm{ml} 99 \mathrm{mTc}\right.$-nanocolloid ${ }^{\circledR}$ suspension) was administered. The intralesional injection was performed under CT-guidance ( $7 \mathrm{pts})$, by using bronchoscopy (5 pts), VAT ( 2 pts) and at time of the thoracotomy ( 8 cases). RT-PCR analysis for cytokeratin-7-19 were used to identify tumor-derived material in $\mathrm{LN}$.

Results: SLN was not identified in 3 pts. SLN was detected in 16 pts. Conventional pathologic examination showed Stage I disease in 13 pts, T3N0 disease in $1 \mathrm{pt}, \mathrm{N} 2$ in 5 pts. The IHC analysis identified lymph-node metastasis in $7(37 \%)$ pts ( 2 evalueted N0 according to H\&E staining). In $6 / 10$ pts underwent to RT-PCR analysis, were detected micrometastasis ( 4 evaluated $\mathrm{N} 0$ according to conventional analysis) Any N0 pts (by RT-PCR staging) had recurrences.

Discussion: SLN technique could provide a subgroup of patients in which the use RT-PCR should be applied on a well-focused target . This approach may be useful for stratifying histologically N0 patients into higher risk and lower risk groups.

\section{Urology}

\section{9}

\section{Percutaneous Nephrolitotomy for Renal Stones: Supine versus Prone Position}

G. Quarto, R. Autorino, S. Mordente, F. Giugliano, F. Neri, C. Quattrone, D. Sorrentino, C. Manzi, R. Muscariello, M. De Sio

Clinica Urologica, AOU Policlinico, Seconda Università degli Studi, Napoli, Italy

Introduction: Our aim was to compare operative time, safety and effectiveness of PCNL in supine vs. prone position. 
Methods: 75 patients (mean age 39.3 years) were prospectively enrolled and randomly divided in two groups: group A (39 pts, supine position) and group B ( $36 \mathrm{pts}$, prone position). Inclusion criteria were: diagnosis of single or multiple renal stones treatable with a single percutaneous access, stone diameter $>2.5 \mathrm{~cm}$, BMI $<30$, no contraindications to perform the operation in prone position. Exclusion criteria were: multiple caliceal stones, complete staghorn stones, coexisting renal anomalies.

Results: The two groups were comparable in terms of age, BMI, $\mathrm{M} / \mathrm{F}$ ratio, stone size. No significant difference was ascertained between the two groups in terms of stone free rate $(88.7 \%$ vs. $91.6 \%$, $\mathrm{p}=0.12)$ blood loss $(\Delta \mathrm{Hb}-2.3 \mathrm{~g} / \mathrm{dl}$ vs. $-2.2 \mathrm{~g} / \mathrm{dl}, \mathrm{p}=0.23)$ and hospital stay ( 4.3 vs. 4.1 days, $\mathrm{p}=0.18$ ). The only significant difference reported was operative time ( 43 vs. $68 \mathrm{~min}, \mathrm{p}<0.001$ ). There was no need of blood transfusion in both groups. No abdominal or thoracic organ injuries were reported.

Conclusions: Supine position showed to be as safe and effective as prone position. Undoubtedly supine position is time saving. We still consider it questionable for complete staghorn stones. PCNL in either position should be part of any endourological training programme.

\section{0}

\section{Bipolar Plasmakinetic versus Standard} Transurethral Resection of the Prostate: Long-Term Follow-up from a Randomized Trial

\author{
S. Mordente, R. Autorino, G. Quarto, F. Giugliano, F. Neri,
}

C. Quattrone, D. Sorrentino, C. Manzi, R. Muscariello,

M. De Sio

Clinica Urologica, AOU Policlinico, Seconda Università degli Studi, Napoli, Italy

Introduction: We evaluated our long-term results from a prospective randomized trial comparing bipolar transurethral resection of the prostate (TURP) with standard monopolar TURP.

Methods: 70 patients had been enrolled: 35 in the bipolar plasmakinetic (Gyrus-ACMI) and 35 in the standard TURP group. The study groups were comparable in age, prostate volume, mean PSA value, IPSS, and quality-of-life score, flow rate, and PVR volume. All the patients completed the 3 years follow-up after TURP and were included in the analysis

Results: The significant improvements in both groups were maintained for the IPSS, QoL score, $\mathrm{Q}_{\max }$ and PVR vs. the baseline values. The main outcome variables at 3 years for bipolar and monopolar TURP were: mean IPSS 6.8 and 6.2; mean $\mathrm{Q}_{\max } 20.5 \mathrm{ml} / \mathrm{s}$ and $21.5 \mathrm{ml} / \mathrm{s}$, respectively. One patient in each group required a second procedure for residual tissue. Bulbar urethral strictures occurred in two patients in each group. Erectile dysfunction was not reported after bipolar TURP, while it affected one patient after monopolar TURP. The retrograde ejaculation rates were similar in both groups.

Conclusions: Our 3 years follow-up confirms our initial positive findings for the efficacy and safety of bipolar PK-TURP.
321

\section{Extracorporeal Shock Wave Lithotripsy (ESWL) for the Treatment of Upper Urinary Tract Calculi (UUTC): Experiences with Mobile Lithotriptor (MI)}

\author{
E. Piazzese1, G.I. Mazzeo', S. Galipòn', F. Fiumara², \\ L.G. Angiò ${ }^{2}$ \\ 1 Unità Funzionale di Chirurgia Laparoscopica, Clinica San \\ Camillo - Messina, ${ }^{2}$ Scuola di Specializzazione Chirurgia \\ Generale I, Università degli Studi di Messina
}

Introduction: ESWL is effective and safe for the treatment of UUTC. To present our results of the past 2 years using a mobile extracorporeal shock wave lithotripter.

Methods: 56 patient (32 female, 24 male; age range 19-81 years) with a total of 136 UUTC were treated by ML. Preoperative evaluation included history, physical examination, routine blood tests, urinalysis, urine culture, renal ultrasound and optional urography. The impulse rate per treatment varied from 750 to 4,000 (mean 3,000). After acute treatment, routine follow-up included renal ultrasound, blood pressure controls, laboratory tests and eventually plain film $\mathrm{X}$-ray.

Results: Successful stones's fragmentation was achieved in all patients; in $43 \%$ were free of stones treated at the time of discharge. At 3-6 months after treatment imaging studies showed no residual fragments in $80 \%$ of the treated. $83 \%$ of the treated stones were cleared entirely. The remaining fragments were clinically insignificant. An average of 3\% ESWL treatments per patient in general anesthesia were required. Ureteral stents were placed in $43 \%$. No significant urinary infection was seen under antibiotic prophylaxis. There was no complication. No change in renal function or blood pressure was found compared to the preoperative values. Hematuria and proteinuria disappeared in all patient who were free of stones.

Conclusions: The results compare favorably with those achieved with fixed lithotriptor and prove that ML are suitable for small centres and fulfils all expectations a user can have in an upt-todate equipment: good disintegration, low side effects, easy handling, fast installation and dual imaging.

322

\section{Tamsulosin versus Nifedipine in Medical Expulsive Treatment (MET) after Extracorporeal Shockwave Lithotripsy (ESWL) in Patients with Distal Ureterolithiasis (DUL)}
E. Piazzese
${ }^{1}$ Unità Funzionale di Chirurgia Laparoscopica, Clinica San Camillo - Messina, ${ }^{2}$ Scuola di Specializzazione Chirurgia Generale I, Università degli Studi di Messina

Introduction: Many studies demonstrate that different drug combinations increase the rate of passage and expulsion of distal 
ureteral stones. We assessed the efficacy of MET with tamsulosin or nifedipine in patients with DUL underwent to ESWL.

Methods: From December 2005 to July 2007, 18 patients (11 female, 7 male; age range 36-64 years) with DUL (calculi size range 6-14 $\mathrm{mm}$ ) after ESWL, were randomly divided into 3 groups. Group A (GA), acted as the control group, included 5 patients; Group B (GB) included 7 patients who received tamsulosin $0.4 \mathrm{mg}$ daily; Group C included 6 patients who received nifedipine $30 \mathrm{mg}$ slowrelease daily. The end points were: stone passage rate, expulsion time and analgesic use (ketorolac).

Results: No significant differences were found between the groups for mean stone size. The stone espulsion rate was $40 \%(2 / 5$ patients) for the GA, $85.7 \%$ (6/7 patients) for the GB and $83.3 \%(5 / 6$ patients $)$ for the GC $(\mathrm{p}<0.05)$. Average expulsion time after ESWL for groups A, B and C was 13,6 , and 7 days respectively $(\mathrm{p}<0.05)$. Finally mean analgesics dosage for the control of pain was significantly lower in the GC and GB especially. 1 patient of the GC has suspended nifedipine a 4 day of therapy for severe ipotension.

Conclusion: From the results of our experience we can affirm that the MET after ESWL for DUL should be considered always; infact Tamsulosin and Nifedipine increased stone expulsion rate, decreased expulsion time and reduced the use of analgesic drugs.

\section{3}

\section{Intraoperatory Radiotherapy (IORT) in Locally Advanced Prostate Cancer (LAPC)}

B. Rocco, B. Jereczek-Fossa, M. Ciocca, A. Vavassori, D.V. Matei, M. Raguso, E. Scardino, F. Verweij, G. Musi, M. Pimentel, S. Detti, R. Orecchia, O. de Cobelli

Istituto Europeo di Oncologia, Milano

Introduction: The adjuvant radiotherapy after radical prostatectomy (RRP) is a standard in LAPC. We estimated the feasibility of IORT with prostate in place during RRP.

Methods: ORT (12 Gy with irradiation of the entire prostate extended to approximately $5 \mathrm{~mm}$ around) and RRP were performed in 23 patients with prostate cancer and probability of organ-confined disease (OC) $<25 \%$ (MSKCC nomograms). Adjuvant RT with $45 \mathrm{~Gy}$ in 25 fractions was performed in patients with stage pT3-4 or pN1. We evaluated intra and post-operating impact in this patients. These results have been compared with those of an historical group of 71 patients with the same clinical characteristics undergone RRP.

Results: The pathological stage evidences OC disease (pT2) in $34.8 \%$ of group IORT and $32.4 \%$ in the group control, with $52.2 \%$ of $\mathrm{N}+$ in group IORT and $18.3 \%$ in the group control. The mean differences on peri-operatory impact has been in terms of surgery duration (median 290' vs. 240'); time to catheter removal was 7 vs. 6 days and length of hospitalization has been $6(5-12)$ vs. 8 (5-16).

Discussion and Conclusions: To our knowledge, our experience is the first report on IORT deliverance before completing RRP. These preliminary data support the feasibility of this treatment. Further studies are needed in order to evaluate the impact of this new technique in terms of long term complications and biochemical disease free survival.

\section{4}

\section{Complications of Slings Incidence and Resolution}

\author{
M.A. Bigozzi*, C. Scalise, O. Dangelo, M. Kobelinsky \\ Uroginecologyc and Pelvic floor Equipment, Policlínica \\ Bancaria - Argentina, Associated hospital to the \\ University of Buenos Aires
}

Introduction: The Stress Urinary Incontinence (SUI) in women is a frequent pathology, whose incidence increases with menopause, with great influence in the quality of life. The surgical correction is the most effective method to treat SUI.

Methods: A retrospective and descriptive study performed from January 2001 to December 2006, on 241 patients operated by SUI, 84 patients through TOT approach and 157 patients with TVT, with average age of 67 years. The complications were divided in intraoperating and postoperating. During the follow up the following studies were performed: anamnesis, physical exam, urine culture, ultrasound with measurement of miccional remainder, urodynamics and urethrocytoscopy.

\section{Results:}

Intraoperatory Complications

TVT: 6 cases $(7 \%)$ of bladder perforations, 1 case $(1.1 \%)$ of intestinal perforation. TOT: 1 case $(0.6 \%)$ urethral perforation, 1 case $(0.6 \%)$ of hematoma.

\section{Postoperatory Complications}

TVT: 3 de novo instability, 6 cases of mesh erosion ( 3 from bladder and 3 vaginal), 1 urinary tract infection, 2 cases of urinary retention.

TOT: 7 cases of de novo instability, 12 mesh erosion ( 1 from bladder, 1 urethral, 10 vaginal), 1 case of urinary retention, 1 case of dispareunia, 1 urethral fistula, 1 cellulitis.

These were solved by means of medical or surgical treatment according to each type of complications.

Discussion: The greater percentage of intraoperating complications was by the retropubic way, whereas during the postoperatory the percentage was similar. Both techniques are easy to perform, with low number of complications, and easy resolution in most cases. We still need to find optimal tape material and construction, as well as a manufacturer.

*miguel.bigozzi@gmail.com

\section{5}

\section{Treatment of Urine Feminine Incontinence: Comparison Between Retropubic Way (TVT) versus Transobturatriz Way (TOT)}

\section{O. Dangelo, M.A. Bigozzi (h)*, C. Scalise, M. Kobelinsky, J. Muhamad}

Uroginecologyc and pelvic floor equipment, Policlínica Bancaria - Argentina

Objective: Compare the effectiveness and complications of the TVT way and TOT way employed to treat stress urinary incontinence (SUI).

Methods: Retrospective and descriptive study performed from January 2001 to January 2006, on a total of 188 operated patients, 74 of whom were operated by SUI and 114 by SUI associated to prolapse. 
Randomized in:

1. Retropubic way (83 cases with urethral hypermobility)

2. Transobturatriz way (105 cases with intrinsic sphincteric deficiency).

The preoperatory evaluations consist on: Physical exam, test of Bonney and urodinamic study. The results were classified as: Successful (total of continence, without losses with the effort), Improvement (smaller incontinence with a satisfactory subjective result) and Failure (those without unsatisfactory clinical improvement); to short (smaller to 3 months), medium (3 to 6 months) and long term (more than 12 months).

Results: The results of both approaches were compared detailing: average age, number of childbirths, previous abdominal surgeries, average lenght of simple surgery and in association to prolapse correction, sanatorial stay, follow up in short, medium and long term. In our experience the long term objective cure was $85 \%$ for the TVT and $86 \%$ for TOT, agreeing with the different international series. The greater percentage of intraoperating complications was by the retropubic way, whereas during the postoperatory they were similar.

Discussion: The successful continence by both routes is similar, and significant differences among them do not exist. These techniques can be associated with prolapsed correction. Although the TOT approach shows less complication, more time of follow up would be necessary to confirm that TOT success, and improvement is similar to the results of the TVT approach.

*miguel.bigozzi@gmail.com

\section{6}

\section{TRIMprob for the Electromagnetic Detection} of Prostate Cancer: Accuracy Evaluation

\section{A. Cicione, C. De Nunzio, S. Squillacciotti, \\ A. Carluccini e L. Miano \\ Dipartimento di Urologia, Ospedale Sant'Andrea, $\|^{\circ}$ Facoltà di Medicina e Chirurgia, Università 'La Sapienza', Roma}

Introduction: TRIMprob is a promising technology for diagnosis of prostate cancer. Aim of our study was to evaluate the accuracy of TRIMprob.

Methods: 111 patients were enrolled. Pts had digital rectal exploration (DRE), evaluation of total and free prostate specific antigen (PSA), and TRIMprob test. Indications for prostate biopsy consisted in PSA levels $>4.0 \mathrm{ng} / \mathrm{ml}$ and/or free/total PSA $<0.15$, and/or positive DRE. TRIMprob resonance levels at $465 \mathrm{MHz}$ were compared between patients with prostate cancer at biopsy and 100 age matched subjects (control group) with PSA levels less than $1 \mathrm{ng} / \mathrm{ml}$. Sensitivity and specificity of TRIMprob and PSA, or TRIMprob and DRE, or PSA and DRE, or PSA and Free/total PSA evaluated together were also performed.

Results: 111 patients were available for analysis. Mean age $64.9 \pm 8.1$ yrs. Mean PSA $8.4 \pm 3.6 \mathrm{ng} / \mathrm{ml}$, free/total PSA $15 \pm 7$. TRIMprob showed a sensitivity of $89 \%$, specificity of $62 \%$ for the diagnosis of prostate cancer, positive predictive value (PPV) of $60 \%$, negative predictive value (NPV) of $89 \%$ and an accuracy of $73 \%$. A significant difference in resonance level at $465 \mathrm{MHz}$ frequency was observed between cancer patients and controls $25.6 \pm 36.8$ vs. $80.7 \pm 48.4 ; \mathrm{p}=0.03)$. The association of two tests does not increase sensitivity and specificity of TRIMprob test.

Conclusions: TRIMProb seems to be a promising technology for early diagnosis of prostate cancer.

327

\section{Photoselective Vaporization of the Prostate (PVP) for the Treatment of Benign Prostatic Hyperplasia (BPH): 1 Year Follow-Up.}

S. Squillacciotti, C. De Nunzio, A. Cicione, A. Carluccini e L. Miano

UOC Urologia, Ospedale Sant'Andrea, Università 'La Sapienza', Roma

Introduction: Aim of our study was to evaluate clinical outcome of PVP for the treatment of BPH.

Materials and Methods: 120 pts enrolled between September 2005 and June 2007 were evaluated by International Prostate Symptom Score (IPSS), Trans Rectal Ultra Sound (TRUS), uroflowmetry, Prostate Specific Antigen (PSA), urodynamic study, urinalysis and urine culture and followed-up at 1, 3, 6, 12 months postoperatively. PVP was performed using a $80 \mathrm{~W}$ KTP laser (Laserscope, USA) and a $23 \mathrm{Ch}$ dedicated endoscope (Storz, Germany).

Results: Mean age was: $69.6 \pm 10$ years. Mean prostate vol. was: $51.9 \pm 20 \mathrm{ml}$ (range 34-132). An average of 261.666 \pm 65.057 $\mathrm{J}$ were delivered over $66.4 \pm 27.8$ minutes. An average of $1.3 \pm 0.5$ fibre were used per patient. Bladder irrigation was required in 10 pts. Mean catheterisation time was $1.3 \pm 0.8$ days. Retrograde ejaculation was reported in $67 \%$ of pts. Clinical outcome is summarized in Table.

\begin{tabular}{lrrrl}
\hline & \multicolumn{1}{l}{ IPSS } & $\mathrm{Q}_{\max }(\mathrm{ml} / \mathrm{s})$ & \multicolumn{1}{c}{ PVR $(\mathrm{ml})$} & PSA $(\mathrm{ng} / \mathrm{ml})$ \\
\hline Baseline & $22.3 \pm 4.0$ & $8.6 \pm 2.7$ & $100.1 \pm 49.8$ & $2.1 \pm 2.9$ \\
1 month & $8 \pm 4.5$ & $23.8 \pm 5.9$ & $18.3 \pm 23.6$ & \\
3 months & $8.9 \pm 4.9$ & $21.1 \pm 4.5$ & $28.9 \pm 21.8$ & \\
6 months & $6.0 \pm 3.8$ & $21.6 \pm 4.8$ & $17.6 \pm 23$ & \\
1 year & $4.4 \pm 3.6$ & $23.7 \pm 4.3$ & $21.4 \pm 16.2$ & $0.9 \pm 0.7$ \\
p $\leq$ & 0.0001 & 0.0001 & 0.0001 & 0.01 \\
Re-operation rate & 6 pts (5\%) & & & \\
Severe ematuria & 4 pts (3\%) & & & \\
Urethral stricture & 6 pts (5\%) & & & \\
Incontinence & 0 pts (0\%) & & & \\
Impotence & 0 pts (0\%) & & & \\
\hline
\end{tabular}

Conclusions: PVP seems to be a mini-invasive but effective procedure for treatment of Lower Urinary Tract Symptoms (LUTS) related to BPH with a good and durable clinical outcome. 


\section{Vascular Surgery}

\section{8}

\section{Carotid Endarterectomy: Regional Anaesthesia vs. Remifentanil Conscious Sedation}

\author{
F. Accrocca, A. Siani, R. Antonelli, G.A. Giordano, \\ G. Marcucci \\ Unit of Vascular and Endovascular Surgery, 'S. Paolo' \\ Hosptital - Civitavecchia - Roma
}

Introduction: To evaluate the safety and efficacy of two different anaesthetic techniques Remifentanil conscious sedation (RCS) vs. loco-regional anaesthesia (LA) in carotid endarterectomy (CEA).

Methods: 154 patients were collected in 2 groups: A RCS and B LA. Group A: induction with propofol $1 \% 1.5-2 \mathrm{mg} / \mathrm{kg}$ e.v. and transmucosal topical application of lidocaine $2 \% 10 \mathrm{ml}$ during tracheal intubation; continuous intravenous $0.12-0.25 \mu \mathrm{g} \cdot \mathrm{kg}^{-1}$. $\mathrm{min}^{-1}$. Remifentanil infusion during anaesthesia in Controlled Mechanical Ventilation. Group B: combined superficial and deep cervical plexus block with Naropine $0.5 \% 25 \pm 5 \mathrm{ml}$. At different times, mean arterial pressure and heart rate were collected. We also assessed the clamping time, intraoperative complications, postoperative morbidity and mortality, shunt insertion, and patient compliance. Anova test for repeated measures and $p$ value $<0.05$ was considered significant.

Results: Haemodynamics variables significant differences were found in group B at basal time $(\mathrm{p}<0.05)$. No significant differences in the frequency of postoperative complications were observed between the two groups (A 5.2\%, B 5.8\% p =0.924). No major strokes in both groups and only one death in the group A.

Discussion: RCS is an innovative technique, monitoring neurological status, as it gives greater assurances of cerebral protection during arterial clamping, better control of the airway.

329

\section{The Role of Arterial and Venous Shunting in the Complex Vascular Trauma of the Arteries of the Lower Limbs}

\author{
A. Siani, F. Accrocca, R. Antonelli, L.M. Siani, \\ F. Mounayergi, G.A. Giordano, G. Marcucci \\ Unit of Vascular and Endovascular Surgery, 'S. Paolo' \\ Hosptital - Civitavecchia - Roma
}

Introduction: Complex lower limb vascular injuries are associated with a high degree of limb loss and an high mortality rate. The purpose of this study was to determine the possible benefits of routine use of a temporary intraluminal arterial shunt.

Methods: From Jannuary 2003 to May 2007, 11 complex blunt and penetrating vascular trauma, with arterial, venous and bone involvement that required stabilization, with lower limb ischemia or bleeding were submit to emergency operation. Over 2 years five artery injuries (3 popliteal, 2 superficial femoral arteries) were managed with insertion of a shunt at the initial phase of the operation (Group B). Data was analyzed and compared with collected data from six complex vascular trauma treated without shunts during the preceding 3 years (group A).

Results: Early shunting of both artery and vein in both penetrating and blunt injuries significantly reduced the incidence of fasciotomies, contracture, nerve plasy, postoperative complications, repeat operations and the incidence of amputation and the mortality rate $(\mathrm{p}<0.05)$.

Discussion: The use of early shunting of artery and vein after lower limb trauma can lead to great advantage in terms of significantly improved outcomes, reducing total ischemic time, ischemic contracture, need to fasciotomy, ischemic nerve plasy and amputation and mortality rates.

330

Thoracic Aorta Stent Graft for Aneurysmal Disease: Lessons Learned
A. Angelini, G. Pagliariccio, A. Medori, P. Persechini,
L. Carbonari
S.O.D. Chirurgia Vascolare, Azienda Ospedaliero - Universitaria Ospedali Riuniti Umberto I - G.M. Lancisi -
G. Salesi, Ancona

Introduction: Endovascular repair for thoracic aortic aneurysms (TEVAR) nowadays represents the treatment of choice in most of the cases of aortic diseases. Introducing hybrid procedures, even complex cases are more easily managed by endografting. However the risk of spinal cord ischemia remains not negligible and unpredictable.

Methods: From 2003 up today, 18 cases of TEVAR are reported. PLZ include zone 3 (15) and zone 2 (3). In one case we performed a visceral arteries revascularization to obtain a satisfactory distal landing zone. Mean length of aortic segment covered by stent graft was $42 \mathrm{~mm}(20-70)$. The graft has been deployed through femoral (12), aortic (5) and iliac (1) access. A coexisting abdominal aortic aneurysm (AAA) was treated by simultaneous endovascular (4) or open repair (2). Previous AAA open repair has been performed in 6 cases. Cerebrospinal fluid drainage was performed in selected cases (previous or simultaneous abdominal aortic surgery, extended coverage of thoracic aorta).

Results: Technical success was achieved in all cases. No inhospital mortality was reported. No major complication were reported. Paraplegia was observed in 3 cases (16\%). Mean follow up was 18 months; type II endoleak rate was $40 \%$ at 1 month, that reduces at $25 \%$ at 12 month. No reinterventions were necessary.

Discussion: TEVAR represents the treatment of choice even for aneurysms involving the aortic arch. It provides a less invasive approach with lower mortality. Selection of cases and an accurate sizing is crucial. Spinal cord ischemia remains the most unpredictable complication despite all different spinal cord protection devices. 
331

\section{Endovascular Repair for Secondary Aorto-Enteric Fistula: A Bridge Solution?}

\author{
A. Angelini, G. Pagliariccio, L. Lavoratornuovo, \\ L. Carbonari \\ S.O.D. Chirurgia Vascolare, Azienda Ospedaliero - \\ Universitaria Ospedali Riuniti Umberto I - G.M. Lancisi - \\ G. Salesi, Ancona
}

Introduction: Secondary aorto-enteric fistula (AEF) represents a life threatening complication of aortic surgery and high rates of mortality are still reported. Graft removal and aortic reconstruction is the treatment of choice. Endovascular repair (EVAR) has been attempted with interesting results. Late failures due to recurrent infection are described.

Methods: From 2004 up to 2006, 6 male patients underwent EVAR for AEF. Patients presented with enteric bleeding and hematemesis and hemodynamic instability (5). An endovascular graft has been deployed in all cases: aorto-iliac (3), bifurcated graft (1), aortic cuff (2). A completion post operative CT scan and upper gastrointestinal endoscopy was performed before discharge. A scanning with Tc-99mHMPAO labeled leukocyte cell, was performed within 3 months.

Results: One patient required early open conversion, extraanatomic bypass and died in 2 nd post operative day. One patient with delayed signs of infection, died after laparotomy for duodenal suturing (bowel ischemia). In all other cases, patients were discharged without major complications; at present none of them presents sign of graft infection.

Conclusions: EVAR may be considered the treatment of choice in patients with AEF presenting with hemodynamc instability; it allows a quick and, in most case, definitive control of acute bleeding. At present EVAR is considered a bridge procedure for acute treatment of AEF, followed by duodenal suture and graft removal. In our experience we avoided early or delayed laparotomy without signs of bowel perforation, graft infection or persistent bleeding.

\section{2}

\section{Urgent Endovascular Treatment for Carotid Plaque Dissection in a Patient with Progressive Stoke. Case Report}

\author{
G. Bianchi, D. Trevisan, S. Canale, R. Curci, R. Felisi, P. De \\ Amicis, A. Argenteri \\ Chair of Vascular Surgery, University of Pavia - Division of \\ Vascular Surgery - Lodi Hospital
}

Introduction: Spontaneous carotid dissection is a rare event (2.6-2.9 cases per $1,00,000$ population), carotid plaque dissection is even more anedoctal in medical literature, making it impossible to determine its real incidence. Plaque dissection can constitute an important cause of stroke in young patients. Often this lesion may be a dynamic process, so that early occlusions may sometimes evolve towards total recanalization or stenosis.

Methods: A 58-year-old male, presented with progressive stroke, with disorientation and right arm weakness, rapidly evolving towards dysarthria, aphasia and right hemisyndrome. Emergent colorduplex revealed an atheromatous plaque dissection at the origin of the left internal carotid, causing preocclusive stenosis. Contrast CT-scan was negative for brain ischemia. The patient underwent left carotid stenting with a Carotid Wallstent.

Results: In the immediate postoperative period remission of aphasia was observed, with restoration of space and time orientation and regression of the right hemisyndrome and of dysarthria.

Conclusions: Due to the paucity of cases reported in literature, it is impossible to extrapolate data regarding morbidity and mortality in the treatment of these lesions. Drawing on the large and recent studies about the treatment of carotid dissection, though, high morbidity and mortality rates of surgery are evident, when compared to elective carotid endarterectomy. Such results cannot be extended to carotid stenting for dissection, which implies morbidity and mortality rates comparable to elective carotid stenting. Due to the lack of invasiveness, rapidity of execution and minimal hemodynamic repercussion of endovascular techniques, this treatment is applicable, at least theoretically, to critical patients.

\section{3 \\ Emergency Endovascular Treatment of Vascular Trauma}

G. Bianchi, D. Trevisan, S. Canale, R. Curci, R. Felisi, P. De Amicis, A. Argenteri

Chair of Vascular Surgery, University of Pavia - Division of Vascular Surgery - Lodi Hospital

Introduction: Blunt vascular trauma is burdened with a dismal life and limb prognosis, due to hemorrhagic shock and the severe vein, bone and nervous injuries typical of these patients. Missed or delayed diagnosis and treatment may therefore jeopardize the patient's life.

Methods: Case 1: female, with huge pseudoaneurysm of the abdominal aorta due to aortic puncture during the positioning of a temporary hemodialysis catheter. Diagnosis, suspected by duplex ultrasound, was later confirmed by angiography. The pseudoaneurysm was excluded by deployment of an aorto-right iliac endograft. Case 2: aortic isthmus rupture following car accident, presented with hemorrhagic shock in a context of polytrauma. The ruptured aortic segment was excluded by means of a straight endograft. Case 3: young patient with multiple precipitation injuries, among which a pseudoaneurysm of the right subclavian artery. Exclusion of the pseudoaneurysm was accomplished by placement of a covered stent.

Results: In all cases, immediate technical success was achieved, with restoration of vessel continuity, cessation of bleeding and restoration of cardiovascular stability. Patients 1 and 2 died after the intervention for severe miocardial disease.

Conclusions: Notwithstanding the technical feasibility of the procedures, results, in terms of survival, have been conditioned by the general condition of the patient (severe cardiovascular impairment), which, in the two fatal cases, has prevailed over the immediate success of the procedure. Our experience, according with literature, refers to a number of cases too limited to allow an objective judgement about the real value of this treatment in the medium and long term. 


\section{4}

From the Need of Carotid Artery Stenting to the Needs in Carotid Artery Stenting: Operators Training and Skill Significantly Reduces Complication Rates. Our Experience

V. Bona, M. Fusari, F. Grillo, P. Trabattoni e P. Biglioli

${ }^{1}$ Centro Cardiologico Fondazione Monzino, Milano, Italia;

${ }^{2}$ Ospedale San Paolo Milano, Italia;

Introductions: Most national or specialty driven guidelines recommend against the use of carotid angioplasty/stenting (CAS) in the management of otherwise low or standard risk patients with asymptomatic carotid disease outwith the protection of randomised trial. However, several registries and single-centre series now include an ever increasing proportion of low-risk asymptomatic patients within their study cohort. Nowadays, CAS procedure has evolved as an effective alternative treatment to carotid endoarterectomy (CEA) due to continual technical advances and operators' training. We report our experience in CAS, focusing on etiology and treatment of proceduresrelated complications.

Methods: The same operator, familiar with ICCS-SPREAD Joint Committee recommendation and qualified according to mandatory training, performed 117 consecutive unselected CAS. Devices technologies and strategies were combined with supra-aortic trunks angiographic anatomy and US plaque morphology, to develop a lesion-specific approach.

Results: Procedural success rate was $100 \%$; peri-operative bradycardia and hypotension was recorded in $41 \%$. Overall follow-up data showed absence of early and late major adverse events (stroke, permanent neurological damage, death) and 3 cases of in-stent restenosis.

Discussion: Due to the devastating potential complications, compared with other endovascular and minimally invasive procedures, carotid stenting requires a much more strict scrutiny of operators' training and outcomes, since the improvement in the learning curve is accompanied by a comparative reduction in the complication rates. We conclude with an appeal for all surgeons and interventionists to share informations about prevention and treatment of procedure related complications, so that future practice is driven by evidence rather than intuition.

\section{5}

Surgical (Transthoracic or Cervical) and Endovascular Repair of the Supraortic Trunk Complex Lesions. A Single-Centre Experience

\author{
A. Bozzani, A. Bocca, F. Ragni, M. Lovotti, A. Odero \\ Divisione di Chirurgia Vascolare, IRCCS Policlinico San \\ Matteo, Pavia
}

Introduction: Reconstruction of the supraortic trunks (ST) can in most cases be performed with either a transthoracic or a cervical approach, or endovascular repair. Patients with severe co-morbidity or candidate to redo-surgery, are also poor candidates for a transthoracic operation.
Methods: Over a 15 -years period, we treated 64 patients (76 total interventions) with ST complex lesion. There were 5 endarterectomies, 27 bypasses, 4 arterial transposition, 8 resection of aneurysm and 32 endovascular procedures. The 'hybrid approach' was required in 5 patients. The innominate artery, common carotid artery and subclavian artery were involved in 5, 28 and 40 patients, respectively. Multiple trunks were reconstructed in 8 patients.

Results: No intraoperative mortality or stent-graft complication were observed. 2 perioperative deaths and 1 nonfatal stroke occurred, for a combined stroke/death rate of $4.6 \%$. Perioperative complications included 1 asymptomatic graft occlusions, 1 nonfatal myocardial infarction, 2 significant pulmonary complications, 1 wound infection, and 2 cranial nerve injury.

Discussion: Direct reconstruction of complex symptomatic ST lesions can be performed with accetable death/stroke rates. Asymptomatic lesions in patients who have significant concomitant conditions should be managed with a less-invasive either cervical or endovascular approach.

\section{6}

\section{A Case of Hypoplasia with Aneurysm of} Infrarenal Abdominal Aorta

\section{A. Bozzani, A. Bocca, F. Ragni, M. Lovotti, A. Odero \\ Divisione di Chirurgia Vascolare, IRCCS Policlinico San Matteo, Pavia}

Introduction: The coarctation of the aorta is one of the most common congenital aortic diseases that almost always occurs in the region of the ductus arteriosus but can be isolated to the abdominal aorta in up to $2 \%$ of cases. A small group of patients showed an infrarenal abdominal aortic aneurysms.

Materials and Methods: A 77-year-old presented with moderate intermittent claudication and chronic renal disease. Physical examination on admission revealed a diminished lower extremities pulses. Contrast arteriography and spiral computerized tomography showed a long narrowing and tortuosity of the infrarenal abdominal aorta associated to an abdominal aortic aneurysm (Ø $50 \mathrm{~mm}$ ). Under general anaesthesia, was performed an aorto-bisiliac bypass (with proximal pipe-beaked anasthomosis) with a $16 \times 8 \mathrm{~mm}$-diameter Dacron graft.

Results: The hysthological finding was fibromuscular dysplasia. The patient postoperative course was uneventful. He was discharged on two antihypertensive medications at the 9th post-operative day.

Discussion: The prognosis of the aortic hypoplasia is generally poor in untreated patients, depending on the severity and complications of the associated hypertension. The treatment of choice is surgical revascularization (resection of the involved segment and end-to-end anasthomosis or placement of an interposition bypass or bypass grafting around the narrowed segment). 
337

\section{Abdominal Aortic Aneurysm Repair in Octogenarians}

N. Troisi, A. Fargion, G. Pratesi, F. Di Carlo, F. Lici,

S. Bellandi, W. Dorigo, C. Pratesi

Cattedra e scuola di specializzazione in chirurgia vascolare, Firenze

Aim: To compare early and late results of open and endovascular repair of abdominal aortic aneurysm in older $(>80 \mathrm{yrs})$ patients.

Methods: From January 2002 to June 2006, 631 patients with AAA were operated on with OR or EVAR; whose 96 were octogenarians. All these patients underwent preoperative assessment to evaluate the feasibility of EVAR. In 42 cases patients were unfit for EVAR and underwent OR (Group 1); in 54 cases EVAR was performed (Group 2) Early and long term results were compared.

Results: Thirty-day mortality was similar in the two groups ( 2 deaths in Group 1 and no death in Group 2,); major complication and reintervention rates were significantly higher in Group 1 ( 5 cases $11.8 \%$ and 4 cases $-9.5 \%$ respectively) than in Group 2 (1 major complication $-2 \%$ and no reintervention, $\mathrm{p}<0.001$ for both parameters). Median duration of follow-up was 21 months; estimated 36-month survival rates were $68.8 \%$ in Group 1 and $59.4 \%$ in Group 2 ( $\mathrm{p}=$ n.s.; $\log$ rank 0.32 ); 36-month AAA-related mortality rates were $3.5 \%$ in Group 1 and $7.7 \%$ in Group 2 ( $p=$ n.s.; log-rank 0.11 ); in Group 1 there was a significantly lower rate of reinterventions than in Group 2 at 36 months ( $9.1 \%$ and $33.2 \%$, respectively; $p=0.03$; log rank 4.6 ).

Conclusions: Older patients anatomically unfit for EVAR can undergo OR with early and long-term results comparable to those obtained with EVAR.

338

\section{A 10-Year Road Towards Risk Reduction in Carotid Surgery}

W. Dorigo, E. Barbanti, A.A. Innocenti, S. Matticari,

I. Spina, S. Massini, N. Troisi, R. Pulli, C. Pratesi

Cattedra e scuola di specializzazione in chirurgia vascolare, Firenze

Aim: To assess the impact of changes in surgical and anesthesiological techniques in last decade on the risk of early complications after carotid surgery.

Methods: Between January 2006 and December 2006, 3,658 consecutive carotid endarterectomies (CEAs) were performed at our Institution. Patients operated on from 1996 to 1998 (971 interventions, Group 1) were compared with those undergone CEA from 2004 and 2006 (1,094 interventions, Group 2).

Results: Interventions were performed under general anaesthesia with somatosensory evoked potentials (SEPs) monitoring and selective shunt insertion in $99 \%$ of cases in Group 1 and in $28 \%$ in Group $2(\mathrm{p}<0.001)$, while the remaining interventions in Group 2 were performed under local anaesthesia. Cumulative shunt insertion rate was $9 \%$ in Group 1 and $12.5 \%$ in Group $2(p=0.04)$. In all the interventions in Group 2 early distal clamping of internal carotid artery was performed, while in Group 1 a standard technique was used. In Group 2 there was a significantly higher number of patch closures than in Group 1. Perioperative results in terms of neurological events, stroke and death were significantly better in the second group; thirty-day stroke and death rates were $1.6 \%$ in Group 1 and $0.5 \%$ in Group 2, $\mathrm{p}=0.01$.

Conclusions: The adoption of a safe surgical strategy allowed us, in last decade, significantly improve our results.

\section{9}

\section{Endovascular Treatment of Peripheral Arterial Disease: Results in TASC II C and D Lesions}
A. Fargion, M. Di Mare, E. Chiti, E. Giacomelli, L. Azas, G. Pratesi, R. Pulli, C. Pratesi
Cattedra e scuola di specializzazione in chirurgia vasco- lare, Firenze

Aim: To evaluate our results in endovascular treatment of PAD in patients with class $\mathrm{C}$ and D lesions on the basis of TASC II classification.

Methods: Between 2005 and 2007, 153 endovascular procedures were performed. Early and midterm results were evaluated. Results in patients with TASC A-B and C-D lesions were compared.

Results: In aorto-iliac district 75 endovascular procedures were performed (53 TASC A-B lesions, 22 TASC C-D lesions). Technical success was $100 \%$ and only one perioperative thrombosis occurred. Median duration of follow-up was 8 months. Estimated 20-month primary and secondary patency rates were $92.5 \%$ and $98.5 \%$, respectively, without TASC-based differences. In femoro-popliteal district 78 endovascular procedures were performed (28 TASC A-B lesions, 50 TASC C-D lesions). Technical success was $95 \%$; in 4 cases conversion to open repair was necessary. Two early thombosis occurred, with a 30-day amputation rate of $1.3 \%$. Median duration of followup was 8 months. Estimated 20 -month primary and secondary patency and limb salvage rates were $57 \%, 73.8 \%$ and $97.4 \%$, respectively. Primary patency was significantly better in TASC A-B lesions than in C-D, but there were no differences in terms of secondary patency.

Conclusions: Endovascular treatment of peripheral arterial disease provides satisfactory early and midterm results also in TASC C-D lesions.

340

\section{A Rare Case of Recurrent Peripheral Embolism Arising from Cardiac Invasion of Lung Cancer}

E. Franciosi, A. Odero Jr, A. Popovich, P. Giorgetti

Chirurgia Vascolare, Istituto Clinico Humanitas, Rozzano

Introduction: There are many etiologies of acute limb ischemia (ALI). Cardiogenic tumour embolisms are rare events: Zurcher in a 
review identified just 38 cases. Invasion of the atrial or pulmonary veins by a tumour mass is a rare event.

Methods: $62 \mathrm{y}$, presenting inoperable, left, stage IIIB non-small cell lung cancer (NSCLC), discovered 3 months previously for deep vein thrombosis (DVT) right poplitea femur, submitted to chemotherapy and undergoing anticoagulant treatment, developed acute left upper limb ischemia from humerus embolism: intraoperative sample of fleshy, white-ish thrombus was analysed. On 2 nd postoperative day new right ALI appeared: returning to normal after disocclusion. Echocardiogram showed stalked mass of the left atrium. Cardiac NMR with gadolinium detected tumour invasion of the atrial wall with mass floating in the atrium. The patient underwent adjuvant radiotherapy:during RT new cerebral ischaemic event with left hemiparesis.

Results: Histology showed tumour cells in the thrombus. No new embolic events were had after RT. Survival was 6 months from diagnosis and 3 months from the first embolic episode.

Discussion: It is known that lung cancer can cause hypercoagulability and thromboembolism. We underline the importance of a mediastinic assessment of suspect rare tumour emboligenic foci in patients without heart disease and with recurring ischaemic episodes.

\section{1 \\ The Hybrid Procedures for Critical Limb Ischemia: Our Experience}

G. Galzerano, P. Sirignano, G. Palasciano, G. de Donato, F. Setacci, E. Chisci, M. Giubbolini, C. Setacci

Chirurgia Vascolare, Siena

Introduction: Aim of this study was to determine safety, clinical improvement, limb salvage of Hibrid Procedures (HP) in patients with CLI (Critical Limb Ischemia).

Methods: Between 2004 and 2006, 631 limb revascularizations were performed, using a primary surgical or a primary endovascular treatment. Otherwise HPs were used to optimize the revascularization in different anatomical districts or to correct an inadequate results of the first procedure.

Results: $10(65 \%)$ endovascular, $76(12 \%)$ surgical and 145 (23\%) HP procedures were performed. In the HP group technical success rate was $98.4 \%$. The cumulative primary, assisted primary, and secondary patency at 6 months were respectively $87.9 \%, 93.4 \%$, $95.2 \%$; the clinical improvement $83.0 \%$; and the limb salvage rates $91.3 \%$. At 1 year the cumulative primary, assisted primary, and secondary patency were respectively $69.6 \%, 71.4 \%, 83.2 \%$; the clinical improvement and the limb salvage rates were $72.1 \%$ and $78.2 \%$.

Discussions: In peripheral multifocal steno-obstructive vascular disease, HP can be an attractive solution, for the reason that the goal of a complete revascularization is not always achievable using separately surgical or endovascular treatment. In our experience the HP is a safe and an effective technique in order to obtain a clinical improvement and a limb salvage for the treatment of CLI. In our centre the HP has become a routine practice.
342

\section{Adventitial Cystic Disease of the Popliteal Artery: Two Cases Report}

P. Sirignano, G. Galzerano, G. Palasciano, G. de Donato, F. Setacci, E. Chisci, M. Giubbolini, C. Setacci

Chirurgia Vascolare, Siena

Introduction: Cystic adventitial disease (ACD) is an uncommon condition with only around 300 cases reported in the literature. We reported two cases of our last five years (2002-2007) experience

Methods: The first patient was a 48-years-old man, ex nicotine abuser, without co-morbidities, with a 4-month history of progressive severe claudication. The second one was a 55-year-old man, ex nicotine abuser, with ischemic heart failure and with a 1-year history of progressive sever claudication. In all cases were performed a duplex scan (dUS) and a CT angiography (CTa), diagnostic for ACD.

Results: In all cases the cysties were incised and the clear, mucinous contents evacuated. The adventitia was repaired and the wound closed. The first patient is currently asymptomatic in the last follow-up (4 years follow-up). The second one at 21 months followup presented newly severe claudication. dUS and CTa demonstrated a cranial progression of the disease (length $=1 \mathrm{~cm}$ ) The narrowed artery was treated with a bare stent and a complete regression of symptoms was obtained.

Discussion: Also be a matter of a rare disease, it goes taken in consideration in young patients with severe claudication and non or poor co-morbidities. In our experience the first treatment is the incision of the cysties and the advential reconstruction. Otherwise short or recurrent lesions can be treated with endovascular therapy.

\section{3}

Renovascular Hypertension. Nine Years Experience of a Vascular Surgery Centre

R. Borghesi, A. Raucci, A. Perulli, G. de Donato, F. Setacci, E. Chisci, M. Giubbolini, C. Setacci

Chirurgia Vascolare, Siena

Introduction: The aim of this study was to evaluate the technical success and clinical outcome of surgical revascularization, angioplasty and/or stenting for renal artery stenosis (RAS) in patients with renovascular hypertension (RVH). The secondary aim was to identify independent negative predictors of blood pressure control after successful renal revascularization.

Methods: From 1998 to July 2007, we treated 105 cases of RAS in $86 \mathrm{RVH}$ patients. Inclusion criteria were RAS $\geq 80 \%$ associated with hypertension refractory to medical control with at least three drugs including a diuretic. Therapeutic options were surgical revascularization in 15 cases and endoluminal treatment in 90 .

Results: Technical success was $100 \%$ for both surgical and endovascular procedures; 14 cases of restenosis were detected: 13 $(14.4 \%)$ in the endoluminal group and one $(6.6 \%)$ in the surgical group $(\mathrm{p}=0.68)$. During the follow-up period, blood pressure control improved in $44 \%$ of patients, disease stabilized in $36 \%$ and the natural course of RVH deteriorated in $20 \%$. A statistical analysis showed that 
only a long history of antihypertensive drug use was a predictor of inefficacy of blood pressure control after revascularization $(\mathrm{p}<0.04)$.

Conclusions: The resolution of RVH associated with severe RAS appears unrealistic in several cases. Results in terms of technical success and restenosis were acceptable and similar for surgical and endovascular renal intervention.

\section{4 \\ Balloon Angioplasty plus Optional Stenting of the Superficial Femoral Artery Obstructions: Our Experience}

\author{
A. Raucci, G. Galzerano, P. Sirignano, G. de Donato, \\ F. Setacci, E. Chisci, M. Giubbolini, C. Setacci
}

Chirurgia Vascolare, Siena

Introduction: To evaluate safe and patency of percutaneous transluminal angioplasty (PTA) plus optional stenting in the treatment of superficial femoral artery (SFA) obstructions in patients with critical limb ischemia (CLI).

Methods: Between 2004 and 2006, 410 endovascular procedures were performed in our center. Of these, 279 procedures were limited to the SFA tract. On the basis of TASC II classification, 68 TASC A, 93 TASC B, 85 TASC C and 33 TASC D consecutive lesions were studied. Nitinol stents were used in case of flow-limiting dissections or $>30 \%$ residual stenosis.

Results: Technical success was obtained in $94.3 \%$ of cases. Stent implantation rate was $67.3 \%$. No deaths at 30 -days occurred. The primary, primary assisted and secondary patency of the SFA at 1-year duplex-US follow-up was respectively: $67.1 \%-83.2 \%-79.6 \%$ (TASC A), 59.4\% - 65.6\% - 57.3\% (TASC B), 53.3\%-55.1\%-51.2\% (TASC C), $42.3 \%-49.7 \%-41 \%$ (TASC D). We had 1 major amputation caused by the procedure (embolization of distal vessels, 4 major bleeding (3 femoral pseudo-aneurysms and 1 SFA perforation). The amputation-free rate was at 1 year $98.7 \%$. Minor amputations occurred on 23 patients $(4.3 \%)$.

Discussions: Balloon angioplasty plus optional stenting of the SFA obstruction is a safe procedure for patients with CLI. Long-term patency rate is dramatically dependent on the basis of the length (TASC A is better than TASC D) and type of lesion (stenosis is better than occlusion).

\section{5}

\section{A Rare Vascular Tumor of the Hand}

\author{
A. Perulli, R. Borghesi, A. Raucci, G. de Donato, F. Setacci, \\ E. Chisci, M. Giubbolini, C. Setacci \\ Chirurgia Vascolare, Siena
}

Introduction: Subungual glomus tumors are rare benign hamartomas arising from the arterial end of the glomus Body.

Case Report: A 45-year-old man presented with a painful, 3 -year-old swelling of the proximal thumbnail of the right hand. The swelling was intensely painful on exposure to cold and pressure. At clinical examination, a slightly bluish nail plate and tenderness under the thumbnail was evident. Hildreth's sign and Love's test were positive. Ultrasonography showed a well-circumscribed hypoechoic area and subtle flow signals within the lesion. Magnetic resonance imaging showed a well circumscribed small oval-shaped mass hyperintense on T2-weighted images, and significant local contrast-enhancement. There was no bone erosion of the distal phalanx. Excisional surgery was performed. After tourniquet placement, the thumbnail was avulsed. A pearly, shiny encapsulated tumor was evident at biopsy. Histology diagnosed a typical, subungual glomus tumor. The patient recovered uneventfully from the procedure: the terrible pain disappeared immediately after surgery and there was no-recurrence at the 1-year follow-up.

Discussion: Whenever a patient suffering from a reddish-purple, tender lesion, usually located at the extremities and especially in the nail bed, with paroxysmal pain and cold-sensitivity, a glomus tumor should be investigated. Surgical exicision is the treatment of choice and it is resolutive.

\section{6 \\ Outcome in Cirrhotic Patients after Surgical Repair of Infrarenal Aortic Aneurysm}

A. Kahlberg, M.M. Marrocco-Trischitta, D. Astore, G. Calori, F. Setacci, G. Melissano, R. Chiesa

IRCCS San Raffaele, Milano

Introduction: Abdominal surgery in patients with advanced liver disease is known to be associated with high morbidity and mortality rates, However, the surgical risk of infrarenal aortic aneurysm (AAA) repair in cirrhotics remains ill defined.

Methods: Between 2001 and 2006, 1,189 patients underwent elective open repair of infrarenal AAA and 24 (2\%) had a biopsyproven cirrhosis $(23 \mathrm{M}$; $1 \mathrm{~F}$; mean age $68 \pm 7 \mathrm{yrs})$. The latter were stratified according to the Child-Turcotte-Pugh (CTP) score and the Model for End-Stage Liver Disease (MELD) score. Operative and perioperative complications were compared to those of 24 matched non-cirrhotic controls.

Results: No intraoperative or 30-day deaths were recorded. No significant differences in terms of major perioperative complications $(p=0.74)$ were observed between groups. Operative time, intraoperative blood transfusion, hospital length of stay were significantly higher in cirrhotics. 22 cirrhotic patients were classified as Child A and two as Child B. Median MELD score was 8 (range 6-14). Both Child B patients died within 6 months. Child's class B and a MELD score $>10$ were associated with reduced midterm survival rates (mean follow-up $27 \pm 24$ months; $\mathrm{p}<0.0001$ and $\mathrm{p}=0.02$ respectively).

Conclusions: In our experience, open AAA repair in cirrhotics was safely performed. However, we noted a reduced life expectancy in Child's class B patients or MELD $<10$. 


\section{7}

\section{Arterial Ulcers: Surgical and Local Management}

A. Kahlberg, E.M. Marone, Y. Tshomba, C. Brioschi, FM. Calliari, D. Logaldo, D. De Dominicis, R. Chiesa

IRCCS San Raffaele, Milano

Introduction: Critical limb ischaemia associated with trophic ulcers is an important risk factor for lower limb loss. Aim of this study is to report a single-Center experience in surgical and local treatment of this specific pathology.

Methods: From 1988 to 2006, 416 patients affected by lower limb arterial ulcers $(332 \mathrm{M}$, age $73 \pm 16$ years) were treated at our Institution. Surgical revascularization has been performed in 324 cases $(78 \%)$. In all patients ( 92 were considered unfit for surgery due to significant comorbidities and/or poor run-off), ulcers have been managed by advanced wound products use, associated with daily 'drop by drop' antiseptic washings (68\%), surgical debridement (22\%) and autologous cultured keratinocytes transplant (7.5\%).

Results: Total wound resolution has been achieved in 212/324 patients who underwent surgical + local therapy $(65 \%)$ and in 9 patients treated with local therapy alone $(10 \%$; $p<0.05)$, with average treatment duration of $3.2 \pm 7.1$ months. Being considered unfit for surgery, the presence of multiple lesions and/or calcaneal lesions, and diagnosis of infection have been found to be predictors of lower resolution rate.

Conclusions: Arterial ulcers of the lower limbs require surgical and appropriate local treatment. Inability to perform surgical revascularization, in spite of an aggressive local treatment, is a risk factor for persistence of the ulcer.

\section{8}

\section{Treatment of Chronic and Acute Carotid Dissections: Experience of $\mathbf{3}$ Cases}

\author{
A. Odero Jr, E. Franciosi, G. Poletto, P.L. Giorgetti \\ Chirurgia Vascolare, IRCCS Istituto Clinico Humanitas, \\ Rozzano
}

Introduction: The best medical or surgical treatments (open/endovascular) of carotid dissections has still not been defined by randomised clinical trials because of the rarity of the disease.

Methods: We recently treated 3 cases (two women and one man, median age 42.3 years) of symptomatic carotid dissection. The first 2 patients presented with chronic dissection of right carotid, undergoing since 2 years anticoagulation treatment became symptomatic for TIA with paraesthesia of the left hemisoma and dysarthria in the first pat. and cephalea with right nuchal pain and left arm paraesthesia in the second case. These pts underwent dissection tailoring surgery and in the second case removal of the intimal flap. The $3 \mathrm{rd}$ pts presented with acute symptomatic carotid dissection for dysarthria and left hemisyndrome (MR and CT scan + ) was treated in emergency. An angioplastic enlargement in pericardius was made for all patients.

Results: The postop. period was uneventful for all pts. Follow up is 12,10 and 6 months.
Discussion: Treatment of chronic and acute carotid dissection is still controversial: anticoagulant therapy does not always protect from further episodes. Even though our study is limited in number, we believe surgical treatment of chronic symptomatic dissections to be necessary and treatment of acute dissections should have the same indications as emergency treatment for carotid lesions.

\section{9 \\ ProCol Vascular Bioprosthesis to Maintain the Patency of the Vascular Accesses}

\author{
F. Benedetto, M. La Spada, F. Mirenda, A. Cotroneo, \\ G. Passari, F. Spinelli \\ Unit of Vascular Surgery, G. Martino University Hospital, \\ Messina, Italy
}

Introduction: We report our experience about the use of ProCol (bovine vein grafts), after prior failed proximal and/or distal accesses, to maintain the patency of arteriovenous fistula for haemodyalisis.

Methods: From January 2004 to December 2006 we performed 32 maintenance operations: 15 (36.6\%) in AVF badly working but not occluded and $17(53.3 \%)$ in AVF occluded. The failure was distal in 10 cases $(30 \%)$ and proximal in $22(70 \%)$. We placed 16 ProCol for stenosis and/or occlusion of arterious or venous tract of the access, showed by color-duplex scanning. Inclusion criteria were: arterious in-flow, venous run-off, absence of sepsi and failed endovascular treatment.

Results: The follow-up at 18 months, by clinical and duplex evaluation, evidenced 2 cases of graft occlusion (12.5\%): in the first we maintain the patency by embolectomy. In the second case, after reintervention, we explanted the ProCol to perform a new vascular access. Never we find infection of the prosthesis. In our experience we used ProCol to replace an arterious and/or venous tract badly working but not as a 'bridge' for puncture.

Discussion: The absence of infection and the mid-long term results demonstrated that the ProCol vascular bioprosthesis is a good alternative to maintain the patency of the badly working vascular accesses if autologous material is not available.

\section{0}

\section{Endovascular Treatment of Vascular Access for Haemodialysis}

A. Pilato', S. Tarantini', M. Leone' ${ }^{1}$, A.M. Maffi ${ }^{2}$, L. Manzo', M. Marini ${ }^{1}$, M. Gessaroli ${ }^{1}$

${ }^{1}$ Unità Operativa Chirurgia Vascolare, Dipartimento delle Malattie Cardiovascolari, ${ }^{2}$ Dipartimento di Radiologia, Ospedale 'Infermi' Rimini

Introduction: To analyze indication and efficacy of endovascular treatment for AVF preservation.

Methods: Between September 2006 and May 2007, 12 male patients underwent endovascular treatment for AVF preservation. Mean age was 73 years (range 62-78). Echocolor-Doppler for vein diameter 
measurement and stenosis evaluation was obtained preoperatively in all patients and stenosis was considered significant for flow reduction less than $250 \mathrm{ml} / \mathrm{min}$. Indications were: anastomotic stenosis of efferent vein or difficulty to complete the dialysis. All patients were followed up with Echocolor-Doppler on a 3-month regular base interval. 4 /12 patients, with proximal AVF, had: anastomotic stenosis $(\mathrm{n}=1)$ and distal basilc vein stenosis $(n=3)$; 4 patients, with distal AVF, had 1 stenosis of cefalic vein $(n=1)$ and multiple stenosis of basilic vein $(n=3)$. 4 patients with PTFE 4-6mm AVF, had: proximal graft stenosis $(\mathrm{n}=1)$, distal $(\mathrm{n}=2)$ and axillary's vein stenosis $(\mathrm{n}=1)$. All the procedures were completed under local anaesthesia only.

Result: Clinical success was achieved in $100 \%$ and the primary patency rate was $100 \%$ and $91.7 \%$ at 1 and 6 months, respectively. One haematoma with complete resolution whithin $24 \mathrm{~h}$ occurred and one prosthetic thrombosis after 5 months.

Conclusions: Endovascular PTA is a safe and effective procedure and it represents a valid alternative to surgery in selected patients.

\section{1 \\ The Impact of Locoregional Anesthesia on Perioperative Stress Response Evoked by Carotid Endoarterectomy (CEA)}

R. Compagna, V. Di Maio, F. Tovecci, A. Bellino, A.K. Markabaoui, P. Sorrentino, B. Amato

Department General Surgery, University 'Federico II' Naples, Chairman: Prof. G. Perisco

Introduction: Carotid endarterectomy (CEA) has been proved to be safe and effective to prevent the risk of stroke in patients with symptomatic or asymptomatic but high grade carotid stenosis. In high risk patients, carotid artery stenting has been proposed as an alternative to CEA, because it is less invasive and avoids surgical trauma. Our aims was to determinate the degree of surgical trauma caused by CEA and to define differences related to the use of locoregional or general anestesia.

Methods: 14 patients were stratified for demographics and risk factors and operated on under locoregional (LA) or general anestesia (GA). Markers of the stress responce (cortisol, adrenocorticotropic hormone, prolactine and $\mathrm{C}$ reactive protein) were measured intraoperatively before and after carotid artery cross clamping (CACC) and postoperatively up to the third day after surgery.

Results: CEA was performed under GA in 8 cases $(55 \%)$ and under LA in 6 (44\%). Intraoperatively cortisol and adrenocorticotropic hormone levels were significantly higher in the LA group. CCA increased the intraoperative cortisol levels in both groups but in patients who underwent in carotid shunting this effect was abolished.

Discussion: Intraoperative surgical stress was higher under LA and was blunted by carotid shunting under both LA and GA.
352

\section{Hybrid Vascular Reconstructions of the Lower Limb: Tradition and Technical Innovation}

\section{Trevisan, G. Bianchi, R. Curci, S. Canale, P. De Amicis, R. Felisi, A. Argenteri}

Chair of Vascular Surgery, University of Pavia - Division of Vascular Surgery - Lodi Hospital

Introduction: Hybrid procedures make simultaneous use of traditional vascular surgical procedures and of endovascular techniques. Must satisfy three conditions: the same aim, a temporal criterion, and the same treated district.

Materials and Methods: In the last twelve months, ten patients with PAOD have been treated. One patient for acute limb ischemia from femoropopliteal bypass occlusion; four patients for critical ischemia; four patients for stage IIB PAOD and one patient for stage IV PAOD from iliac obstruction and complete occlusion of the AFS. In four cases, endovascular procedures were used to improve inflow; in four cases to improve outflow; in two cases was treated a distal anastomotic stenosis.

Results: In all cases, the completion angiographic findings have been satisfying. A good clinical result was present in five cases at over six-month follow-up. Two cases have suffered early occlusion (within 48 hours) of the femoro-popliteal axis; the first patient was compensed with vasoactive drugs, in the second patient major amputation was performed. In one case, the hemodynamic result of iliac angioplasty was not satisfying, so a femoro-iliac endarterectomy was performed. In one case, a very tight restenosis of the tibioperoneal trunk developed, and a cryoplasty was performed, with good immediate clinical and duplex scan result. In one case, two months after PTA of the distal anastomosis, acute ischemia from graft thrombosis was observed.

Discussion: Hybrid vascular reconstructions allow broadening of surgical indications both on a clinical standpoint and a morphologic one. Run-in and run-off improvement favorably affect the primary and the secondary patency rate. 


\section{Author Index for Abstracts}

\section{European Surgical Research}

Aboh, I.V., 175, 184

Accrocca, F., 328, 329

Agliardi, A.C., 250, 252, 253, 254

Agresti, M., 86, 87, 88, 89, 273

Agrusti, S., 63, 64

Albertazzi, A., 10

Aldini, R., 280

Alfano, C., 261

Alì, A., 196

Ali Youssef, D., 176

Alimenti, E., 22

Allaix, M.E., 164, 165

Altimari, A., 17

Amaduzzi, A., 19

Amato, B., 54, 55, 60, 61, 62, 72, 73, 114, 115, 125, 275, 351

Ambrosio, G., 177, 185

Amore, A., 65

Amoroso, R., 200

Andorno, E., 21

Andrea, M., 239

Andreoni, B., 208

Andria, B., 22

Angelini, A., 330, 331

Angelini, L., 158, 160, 161, 162

Angelini, M., 263

Angeloni, E., 4, 5, 6

Angiò, L.G., 108, 109, 110, $111,112,168,169,170$, $171,321,322$

Annacontini, L., 265, 266, 267, 268, 269

Ansaloni, L., 30, 34, 124, 206, 284

Antinolfi, L., 78

Antonelli, R., 328, 329

Antonellis, F., 66, 67

Antonino, A., 114, 115, 117

Aprea, G., 74, 75, 149

Aquino, F., 237

Araco, F., 140

Aragiusto, G., 14, 22

Ardò, N.P., 285, 317

Argenteri, A., 332, 333, 352

Argnani, D., 302, 303

Armaro, A., 2

Armellino, M., 32

Arnoldi, R., 249, 253, 254
Numbers refer to abstract numbers
Arpesella, G., 9

Arzu, G., 12, 52, 79, 118

Astarita, E., 236

Astore, D., 346

Atzeni, J., 44

Auriemma, G., 140, 141

Autorino, R., 319, 320

Avalli, L., 7, 8

Aversa, C., 183

Azas, L., 339

Bacchini, M., 2

Bagnato, C., 85, 217

Baisi, A., 309

Baj, A., 176, 178, 179

Balato, G., 236

Ballarin, R., 12, 52, 79, 118

Bandiera, A., 296, 297

Barabino, G., 21

Barbanti, E., 338

Barlettai, M., 84, 85, 103, 104, 217

Barone, D., 222

Barra, L., 119

Bartoli, A., 147

Bartoli, D., 194

Bashkcon, S., 251

Basso, G., 266, 267

Batoli, A., 167

Battaglia, B., 207, 209

Battisti, A., 175, 184

Belardi, A., 209, 211

Bellandi, S., 337

Bellantone, R., 43

Bellezza, F., 57

Belli, A., 62

Bellino, A., 54, 72, 125, 351

Bellò, M., 213

Bellochi, R., 167

Bellotti, A., 274

Bellotti, V., 235, 236

Beltempo, P., 280, 281

Beltramini, G., 178

Beltramo, G., 213

Benato, C., 295

Bencini, L., 143

Benedetto, F., 348

Benedetto, U., 4, 5

Benetti, D., 304, 305

Benozzi, L., 10

Benvenuti, L., 200
Benvenuti, M., 304, 305

Berardi, A., 204

Bernini, M., 143

Berra, A., 286

Bertelli, R., 9, 10, 11, 29, 34, 280, 281

Berti, P., 98

Bertoglio, C., 144, 270

Bertolaccini, L., 286

Bettarini, F., 205

Biancafarina, A., 148

Bianchi, E., 11, 280

Bianchi, G., 332, 333, 352

Bianchi, P.P., 153

Bianchini, R., 4

Biasco, G., 206

Biffi, R., 208

Biglioli, P., 334

Bigozzi, M.A., 324, 325

Bini, A., 290

Biondani, G., 295

Biondi, A., 214, 215, 216

Biondi, G., 65

Birolo, S., 164

Bisceglia, P., 266

Bistoni, G., 283

Bittesin, L., 120

Bocca, A., 335, 336

Bocchiotti, M.A., 256, 257

Bogetti, P., 256

Bona, S., 153

Bona, V., 334

Bonadiman, C., 291

Bondurri, A., 68

Bongini, G., 37, 38, 71

Boni, L., 145, 146

Bonora, S., 301

Bonsignore, A., 110

Borghesi, R., 342, 345

Borghi, F., 36

Borsato, N., 120

Bortl, M., 121

Boscherini, M., 43

Bossola, M., 43

Botta, L., 1, 2

Botticini, G., 304, 305

Bottino, G., 21

Bottino, V., 166

Bove, P., 191

Bovolato, P., 304, 305

Bozzani, A., 335, 336
Bracco, A., 22

Brachet Contul, R., 24, 25, 26, 27,28

Brancaccio, U., 81, 154

Brandolini, J., 290

Braun, A., 117, 276

Brauner, E., 175, 184

Brescia, A., 158, 159, 160, 161, 162

Brillantino, A., 126, 127

Brioschi, C., 347

Brunaccino, R., 156, 157

Brunelli, F.G., 3

Bruschi, S., 256, 257

Brusciano, L., 119, 173, 174

Brustia, R., 100

Bruzzi, C., 225

Bruzzo, M., 195

Bucci, L., 78

Bucci, T., 177, 185

Bugiantella, W., 69, 70, 278

Bulf, M., 313

Bulla, A., 255, 264

Busi, N., 10

Cacciapuoti, M., 74, 75, 149

Cacciapuoti, T., 116

Calabrese, D.P., 271

Calabrese, F., 301

Calabrese, L., 186, 187, 188

Calabria, R., 96

Calabrò, F., 295

Calati, A.M., 309

Calenda, M., 141

Caletti, G., 206

Caliceti, U., 225

Califano, L., 177, 185

Calise, F., 14, 22

Calliari, F.M., 347

Calò, P.G., 44

Calori, G., 346

Calzolari, F., 222

Campanale, A., 267, 268, 269

Campanelli, G., 63, 64

Campione, M., 65

Campisi, G., 191

Camurri, N., 1, 2

Canale, S., 332, 333, 352

Cananzi, F.C.M., 214, 215, 216

Cancian, L., 310, 311

Cancilleri, F., 242

\section{KARGER}

(C) 2008 S. Karger AG, Basel and IAP

Fax +4161306 1234

E-Mail karger@karger.ch

www.karger.com

Accessible online at:

www.karger.com/journals/esr 
Cantarella, F., 70

Cantisani, R., 84, 85, 103

Cantore, F., 145, 146

Canziani, M., 63, 64

Capasso, S., 156, 157

Capocasale, E., 10

Capozzi, R., 288, 289, 306

Cappabianca, P., 198, 199

Cappelli, G., 10

Capuano, F., 4

Capuano, G., 31, 150

Carbonari, L., 330, 331

Cardamone, B., 35

Carlini, M., 152

Carlomagno, N., 136, 137

Carlucci, S., 256, 257, 258, 259, 260

Carluccini, A., 326, 327

Carretta, A., 296, 297

Caruso, S., 204, 205

Casaccia, M., 21

Casciola, L., 147, 148, 167

Casiraghi, M., 296, 297

Cassano, L., 227

Cassese, M., 180

Cassinotti, E., 145, 146

Cassioli, V., 56, 57, 58, 59

Cassoni, A., 194

Cassoni, P., 212, 213

Castellano, I., 212

Castello, E., 196

Cataldo, C., 32

Catarci, M., 95, 207, 209, 210, 211

Catena, F., 29, 30, 34, 124, 206, 284

Catena, N., 230, 231, 232, 233

Caudana, R., 291

Causa, N., 77

Cautero, N., 13, 20, 23, 132

Cavaliere, D., 218, 219, 220, 221, 222

Cavaliere, M., 223, 224

Cavallari, G., 9, 10, 11, 279, 280, 281

Cavallaro, A., 282

Cavallaro, G., 42, 282

Cavalli, M., 63, 64

Cavallo, L.M., 199

Cavazzoni, E., 69

Cavazzuti, A.M., 255

Cavicchi, O., 225

Ceccarelli, G., 147, 148, 167

Cecchini, S., 101

Cecere, D., 53

Celiento, M., 114, 116, 275

Celsi, S., 81, 154

Cenciarelli, S., 208

Cerbone, D., 78

Ceriello, A., 14, 22
Cerullo, G., 205

Cescon, M., 15, 16, 18, 19, 34

Chen, H.C., 262

Chessa, A., 71

Chiaverini, R., 65

Chiesa, R., 346, 347

Chiriotto, M., 212

Chisci, E., 341, 342, 343, 344, 345

Chiti, E., 339

Chiummariello, S., 261

Cicione, A., 325, 327

Cigna, E., 262, 283

Ciocca, M., 323

Cioffi, G., 77

Ciolli, M.G., 135, 137

Ciriaco, P., 296

Cirillo, C., 74, 75, 116, 117, 149, 276

Cirillo, M., 3

Civitelli, F., 71

Clemente, M., 137, 138, 139

Cloro, L., 106

Cloro, P.M., 106

Coccolini, F., 30, 124, 206, 284

Cognetti, C., 86, 87, 88, 273

Colella, G., 191

Comandi, A., 58, 59

Comito, C., 5

Compagna, R., 54, 55, 60, 61, $62,72,73,125,276,351$

Conforti, S., 287

Conte, F., 283

Conzo, G., 81, 154

Coppola Bottazzi, E., 74, 75, 149

Corcione, F., 151

Cornaglia, S., 100

Corriero, O.V., 198

Corso, G., 204, 205

Corti, B., 17

Corti, F., 8

Corzo, L., 190

Coscia, V., 255

Cosentino, L.M., 209

Cosenza, A., 126, 127

Cosenza, M., 159

Costa, V., 243

Costi, R., 101

Cotroneo, A., 349

Cremone, C., 35, 76

Crisman, G., 121

Cucchetti, A., 15, 16, 18, 19

Cuccurullo, D., 151

Cucina, A., 282

Curci, R., 332, 333, 352

Cuttitta, D., 129, 130, 131

Da Broi, J., 24, 25, 26, 27, 28

D'Acapito, F., 221
Daddi, G., 288, 289, 306

Daddi, N., 288, 289, 306

Dal Conte, I., 212

D'Alessandro, L., 29, 30, 34, 124, 206

Dalla Valle, R., 10

D'Ambra, M., 157

Damiano, I., 166

D'Andrea, A., 195, 196, 197

D'Andrea, L., 230, 231, 232

Danesi, T.H., 4, 5, 6

Dangelo, O., 324

D'Angelo, S., 135, 136, 137 , 138,139

D'Antonio, D., 77, 78

Datta, G., 256, 258, 259

Davoli, F., 290

Dazzi, A., 15, 16, 17, 18, 19

De Amicis, P., 332, 333, 352

De Angelis, C., 20

De Biase, S., 185

De Biasi, S., 177, 181, 182

De Blasiis, M.G., 12, 52, 79, 118

De Bonis, P., 229

de Cobelli, O., 323

De Corso, E., 229

de Divitiis, O., 198, 199

De Dominicis, D., 347

de Donato, G., 341, 342, 343 , 344,345

De Giulio, F., 177

De Luca, A., 270

De Luca, S., 13, 20, 23, 132

De Magistris, L., 115, 276

De Marco, G., 204, 205

De Martini, P., 144

De Martino, C., 31, 39, 150

De Masi, A., 32

de Matteis, V., 300

De Nunzio, C., 326, 327

De Palma, A., 285, 307, 314, 317

De Palma, G., 72, 73

De Prizio, M., 155

De Ruvo, N., 12, 52, 79, 118

De Sio, M., 319, 320

De Stefano, G., 32

De Toma, G., 42, 282

De Vinci, C., 281

De Vita, D., 140, 141

De Vito, D., 55, 117, 276

de Werra, C., 107

Defez, M., 14

Del Gaudio, M., 15, 16, 18, 19

del Genio, A., 119, 173, 174

del Genio, G., 119, 173, 174

Della Beffa, V., 286

Della Corte, M., 33

della Libera, D., 120
Della Pietra, C., 154

Della Rocca, P., 151

Dell'Agnola, C.A., 251

Dell'Amore, D., 302, 303

Dell'Avanzato, R., 56, 57, 58, 59, 152

Dell'Aversana Orabona, G., 177

Delli Carpini, A., 65

Delmonte, S., 212

Denaro, V., 238, 239, 240, 241, 242, 243

D'Errico, A., 16, 17

Desiderio, S., 226

Detti, S., 323

Di Bartolomeo, R., 1, 2

Di Battista, M., 206

Di Benedetto, F., 12, 52, 79, 118

Di Carlo, F., 337

Di Cintio, A., 95, 207, 209, 210, 211

Di Falco, G., 310, 311

Di Francesco, A., 193

Di Francesco, F., 13, 20, 23, 132

Di Frattta, E., 156

Di Giuseppe, M., 107, 145, 146

Di Gregorio, A., 95, 211

Di Lauro, S., 107

Di Liberto, C., 191

Di Lieto, C., 225

Di Lorenzo, G., 226

Di Maio, V., 55, 60, 61, 62, 73, 117,351

Di Marco, L., 1

Di Mare, M., 339

Di Marino, M., 74, 75, 149

Di Martino, Alberto C., 239

Di Martino, G., 271

Di Martino, M., 205

Di Martino, N., 126, 127

Di Micco, R., 107

Di Napoli, G., 234, 235, 237

Di Napoli, M.G., 245, 246, 247, 248, 249, 250, 251, 252, 253, 254

Di Nucci, G., 4, 5

Di Nucci, G.D., 6

Di Sandro, G., 65

Di Sandro, S., 12, 52, 79, 118

Di Saverio, S., 29, 34, 284

Di Scioscio, V., 206

Di Silverio, P., 156

Di Simone, M., 15, 17, 18

Di Stazio, C., 122, 123

Diamantis, G., 50, 51

Dilillo, S., 156, 157

Dionigi, G., 145

Diurni, M., 145 
Dobrinja, C., 80

Docimo, G., 45, 98, 277

Doglietto, F., 229

Dolci, G., 290

Domatsoglou, A., 261

Donatini, G., 98

Donato, G., 201

Donini, A., 69, 70, 278

Donzelli, I., 107

Dorigo, W., 337, 338

Drago, S., 95, 274

Droghetti, A., 291

Dubini, A., 222

D'Ugo, D., 214, 215, 216

Duranti, L., 292

Eccher, C., 133

Elmore, U., 153

Ercolani, G., 9, 15, 16, 18, 19, 34

Esposito, A., 39, 226

Esposito, E., 271

Esposito, F., 198, 199

Esposito, I., 199

Esposito, M.G., 81, 154

Fabozzi, M., 24, 25, 26, 27, 28

Facciuto, E., 180

Faenza, A., 9, 10, 11, 279

Famà, F., 41, 82, 83, 99

Famà, R., 133

Fania, S., 227

Fanucchi, O., 292, 318

Farace, F., 255, 264

Fargion, A., 337, 339

Farina, A., 271

Farris, S., 44

Fattori, R., 1

Fausto, F., 134

Favi, F., 158, 160, 161, 162

Fede, A., 230, 231, 232, 233

Fedeli, C., 289, 306

Fedeli, S., 250

Federico, G.L., 237

Fei, L., 129, 130, 131

Feil, B., 295

Felisi, R., 332, 333, 352

Feng, G.M., 262

Ferla, L., 297

Fernandez, I.J., 225

Feroci, F., 155

Ferrante, D.R., 209

Ferrari, C., 21

Ferrario, F., 192

Ferraro, G., 201

Ferro, O., 8

Ferronato, A, 298, 299

Ferulano, G.P., 156, 157

Fiamingo, P., 133

Fico, D., 156, 157
Fieschi, S., 287

Filidei, F., 84, 85, 103, 104, 217

Fini, M., 284

Fini, N., 266

Fiorani, B., 4, 6

Fiorello, A., 293, 294

Fiumara, F., 108, 109, 110, 111, $112,168,170,171,321,322$

Fiume, I., 35

Fogacci, T., 272

Folli, S., 302

Fontana, P., 298, 299

Fontani, A., 71

Forcella, D., 315

Forestieri, P., 128, 172

Formato, A., 128, 172

Formica, F., 7, 8

Formillo, P., 179

Formisano, C., 271

Fornasier, V.L., 241

Foroni, F., 86, 87, 88, 89, 273

Fosso, A., 98

Fraccalvieri, M., 256

Framarini, M., 218, 219, 220, 221, 222

Franciosi, E., 340, 348

Frati, S., 289

Frattini, F., 63, 64

Frigerio, A., 144

Friscia, M., 185

Frustaci, G., 98

Fuga, G., 9

Fumagalli, U., 153

Furia, S., 295

Furlan, N., 153

Fusari, M., 334

Fusaroli, P., 206

Gagliardi, R., 200

Gagliardi, S., 34, 284

Gaj, F., 66, 67

Galassi, R., 218

Galatà, G., 46, 47, 48, 49, 90

Galatioto, C., 85

Galipò, S., 108, 109, 111, 112, $168,170,171,321,322$

Gallazzi, M., 316

Galli, V., 226

Galloro, G., 50, 51

Galzerano, G., 341, 342, 344

Garelli, P., 13, 20, 23, 132

Garofalo, G., 271, 314

Gasparrini, M., 158, 160

Gatti, C., 121

Gavioli, M., 34

Gazzabin, A., 56

Gazzabin, L., 56, 57, 58, 59

Gazzotti, F., 30, 34, 124, 206, 284

Gennaro, P., 184
Gerunda, G.E., 12, 52, 79, 118

Gervasoni, C., 189, 193

Gessaroli, M., 350

Ghinassi, S., 207, 210, 211

Ghiribelli, C., 308, 316

Giacomelli, E., 339

Giacometti, C., 301, 312

Giannì, A.B., 176, 178, 179, 192

Giannini, G., 265, 267

Giardino, R., 284

Giavarini, L., 145, 146

Gili, S., 45, 91, 277

Gilio, F., 86, 87, 88, 89, 273

Gilioli, C., 10

Gioffrè-Florio, M.A., 41, 82, $83,99,134$

Giordano, G.A., 328, 329

Giordano, V., 7

Giorgetti, G., 220

Giorgetti, J., 200

Giorgetti, P., 340

Giorgetti, P.L., 348

Giovanardi, M., 297

Girardi, V., 114, 117, 275

Giraudo, G., 36, 164

Giubbolini, M., 341, 342, 343, 344,345

Giubrone, D., 230

Giuffrida, M.C., 36

Giugliano, F., 319, 320

Giuliani, A., 14, 22

Giuliano, E., 166

Givigliano, F., 200

Golfieri, L., 15, 16, 18, 19

Gotti, G., 308, 316

Gozzo, G., 265, 268

Granato, F., 308, 316

Grandi, S., 19

Grandinetti, P., 106

Grassi, C., 208, 221

Grassi, G.B., 95, 207, 209, 210, 211,274

Grassi, I., 37, 38

Grassi, V., 37, 38

Grassia, R., 223, 224

Grassia, S., 135

Grazi, G.L., 9, 15, 16, 18, 19

Graziano, P., 185

Graziosi, L., 69, 278

Grieco, M., 265, 267, 268

Grigioni, F.W., 16

Grillo, F., 334

Grillo, G., 107

Grosso, E., 234

Guadagni, I., 122, 123

Guadalaxara, A., 163

Guarino, R., 31, 150

Guarino, S., 235

Gubitosi, A., 86, 87, 88, 89, 273
Guerrini, G., 52, 118

Guerrini, G.P., 79

Gurrado, A., 92, 93, 94, 113

Iaccarino, V., 81

Iacomino, E., 180

Iacono, A., 77

Iacono, V., 234, 236

Iannone, L., 6, 115, 275

Iengo, M., 223, 224

Iera, M., 261

Impallomeni, C., 231, 233

Innocenti, A.A., 338

Insabato, L., 78

Inzirillo, M., 50, 51

Iofrida, G., 201, 202, 203

Ioia, G., 39

Ionna, F., 183

Iovino, F., 91, 277

Ippolito, M., 242

Irlandese, E., 45, 91, 277

Iside, G., 86, 87, 88, 89, 273

Iuvone, T., 105

Izzo, D., 126, 127

Izzo, G., 126, 127

Jereczek-Fossa, B., 323

Kahlberg, A., 346, 347

Kang, James D., 240

Kimura, T., 15, 16, 18

Kirienko, A., 244

Kobelinsky, M., 324, 325

Kroning, K., 155

La Barba, G., 15, 16, 18, 19

La Spada, M., 349

Laganà, F., 176, 179, 192

Lambert, Y., 205

Lanero, L., 182

Langone, M.A., 226

Lanoce, E., 106

Laperuta, P., 293

Lardo, D., 92, 93, 94, 113

Latorre, G., 43

Lauro, A., 9, 15, 16, 17, 18, 19, 34

Lavermicocca, W., 94

Lavoratornuovo, L., 331

Lazzarott, T., 15

Lehmann, R., 279

Lenna, G., 68

Leonardi, F., 238

Leone, A., 1

Leone, L., 95, 207, 209, 210, 211

Leone, M., 350

Leparulo, L., 183

Leporati, A., 309

Libretti, L., 296, 297 
Liccardo, L., 181, 182

Lici, F., 337

Ligabue, T., 304, 305

Liguori, G., 80

Limite, G., 271

Limongelli, P., 122

Linard, C., 41, 82, 83, 99, 134

Lionetti, R., 156, 157

Lippolis, P.V., 217

Lissidini, G., 92, 93, 94, 113

Livrea, A., 81, 154

Lo Giudice, F., 298, 299

Lo Schiavo, F., 45, 91, 277

Loffredo, A., 24

Logaldo, D., 347

Loizzi, D., 285, 307, 314, 317

Loizzi, M., 307, 314

Lombardi, C.P., 43

Lombardi, D., 39

Lombardi, G., 39

Lombari, P., 135, 137

Lomonaco, A., 288, 289, 306

Longo, A., 184

Longo, B., 263

Longo, C., 118

Longo, F., 183

Longo, G., 175, 211

Lorè, B., 186, 187, 188

Lovadina, S., 298, 299

Lovotti, M., 335, 336

Loy, M., 312

Luca, F., 208

Lucchi, M., 292

Lucernoni, P., 311

Lucia Giovanna Maria di Somma, 199

Lucido, F., 173, 174

Lucisano, A.M., 96, 97

Luglio, G., 78

Lullo, D., 140, 141

Lunardi, F., 301, 312

Luzzani, S., 248

Luzzi, L., 308, 316

Macchini, F., 245, 246, 247

Maduri, R., 201, 202, 203

Maffettone, V., 173, 174

Maffi, A.M., 350

Magno, L., 50, 51

Maida, P., 22, 166

Maietta, M., 185

Maietta, P., 299

Maiorella, A., 266, 267, 269

Maiorino, A., 300

Maira, G., 229

Malinconico, F.A., 86, 87, 88, 89, 273

Mancini, R., 84

Mancini, S., 56, 57, 58, 59, 210

Mancini, St., 56, 57, 58, 59
Mangone, G.M., 180, 182

Manno, E., 286

Manzi, C., 319, 320

Manzo, L., 350

Marcucci, G., 328, 329

Maresca, A., 40

Mari, F.S., 158, 159, 160, 161, 162

Marianetti, T.M., 194

Mariani, G., 186

Mariani, P., 163

Marinelli, P., 120

Marini, M., 350

Marinò, D., 237

Marinozzi, A., 242

Markabaoui, A.K., 60, 62, 73, 351

Markaboui, K.A., 54

Marone, E.M., 347

Marrelli, D., 204, 205

Marrocco-Trischitta, M.M., 346

Martelucci, J., 71

Martinelli, N., 238, 242, 243

Martini, A., 40

Martini, F., 143

Martino, A., 7, 8

Martino, A.D., 243

Martin-Suarez, S, 2

Martorelli, G., 13, 20, 23, 132

Marulli, G., 301, 312, 313

Marvaso, A., 77

Marzaioli, R., 93, 94

Masetti, M., 12, 52, 79, 118

Masi, A., 14

Masi, G., 36

Masia, D., 263

Masone, S., 73, 74, 75, 149

Masoni, L., 158, 159, 160, 161, 162

Massagglia, F., 286

Massariello, D.N., 265, 269

Massi, F., 1

Massimo, M.R., 300

Massini, S., 338

Mastrobuoni, G., 74, 75, 149

Mastrodicasa, E., 274

Mastrorilli, G., 40

Mastrorilli, M., 40

Matei, D.V., 323

Materazzi, G., 98

Matteucci, F., 218

Matticari, S., 338

Maura, A., 46, 47, 48, 49, 90

Mazzei, S., 41, 82, 83, 99, 134

Mazzeo, G.I., 108, 109, 111, $112,168,170,171,321,322$

Mazzeo, L.A., 198

Mazzillo, M., 84, 104

Mazzoni, M.P., 10

Meda, S., 309
Medori, A., 330

Melfi, F.M.A., 318

Melina, G., 5

Melis, A., 44

Melissano, G., 346

Meloni, M.C., 238, 241, 242

Melotti, G.L., 102

Menconi, G.F., 200

Mengozzi, M., 302, 303

Mentone, A., 185

Merciadri, P., 195

Mesolella, M., 226

Messina, A., 3, 199

Mhagna, Z., 3

Miano, L., 326, 327

Miccoli, P., 98

Miceli, A., 4, 5, 6

Miklosova, Z., 17, 18

Mikus, E., 1, 9

Mikus, P.M., 9

Milanesi, E., 213

Milillo, A., 158, 159, 160, 161, 162

Millo, P., 24, 25, 26, 27, 28

Milone, M., 31, 150

Mineo, T.C., 315

Mingione, A., 115, 116, 117

Mingrone, P., 106

Miranda, E., 143

Mirenda, F., 349

Mistrangelo, M., 100, 164, 165, 212, 213

Mobiglia, A., 213

Moccia, F., 129, 130, 131

Moccia, G., 86, 87, 88, 89, 273

Monarca, C., 261, 283

Monda, A., 172

Monestiroli, U.M., 68

Montalti, R., 12, 52, 79, 118

Montano, N., 229

Montemurro, L., 210

Monteverde, M., 302, 303

Monti, G.N., 14

Montorsi, M., 153

Moraldi, L., 143

Morana, G., 310, 311

Mordente, S., 319, 320

Morelli, D., 22

Morelli, N., 21

Moretti, A., 218

Moretti, R., 143

Morino, M., 164, 165

Moritz, W., 279

Mosca, S., 289

Mottola, A., 39

Mounayergi, F., 329

Muccione, M.C., 141

Muhamad, J., 325

Mulas, P., 255, 264

Munegato, G., 120
Mura, G., 218, 219, 220, 221, 222

Muriana, G., 291

Muriana, P., 291

Murrone, D., 202, 203

Muscariello, R., 319, 320

Musella, M., 31, 150

Musi, G., 323

Mussa, A., 100, 212, 213

Mussi, A., 292, 318

Nacchiero, L., 92, 93, 94, 113

Napolitano, E., 157

Napolitano, F., 294

Napolitano, M.M., 166

Napolitano, V., 173, 174

Nardi, M., 25, 26, 27, 28

Nardi, M. Jr, 24

Nardo, B., 9, 10, 11, 29, 279, 280, 281

Nardone, S., 4

Neri, A., 204, 205

Neri, F., 11, 280, 281, 319, 320

Nicolai, G., 186, 187, 188

Nicolini, D., 13, 20, 23, 132

Nicolò, D., 245, 251, 252

Nicolosi, A., 44

Nicotra, S., 313

Odero, A., 335, 336

Odero, A. Jr, 340, 347, 348

Onesti, M.G., 283

Orabona, G., 107

Orecchia, R., 323

Orvieni, V., 68

Pacca, G., 45

Pacilè, V., 110

Pacini, D., 2

Pafundi, D.P., 96, 97

Pagan, V., 298, 299

Paganelli, M.T., 70

Pagliariccio, G., 330, 331

Pagliarulo, V., 181, 182

Paladini, P., 316

Palasciano, G., 341, 342

Palazzo, A., 81, 154

Palazzolo, V., 189, 193

Palladino, A., 101, 102

Palma, E., 271

Palma, F., 141

Paludetti, G., 229

Palumbo, G., 128

Panaro, F., 21

Panicucci, S., 103, 104, 217

Pantaleo, M., 206

Paolini, G., 7, 8, 263

Paparelli, C., 282

Pariali, M., 280

Parini, U., 25, 26, 27, 28 
Pariscenti, G.L., 304, 305

Parmeggiani, D., 87, 105, 142, 273

Pascariello, A., 50, 51

Passali, G.C., 229

Passari, G., 349

Pastena, F., 77

Pastore, A., 50, 51

Pata, F., 96, 97, 106

Patriti, A., 147, 148, 167

Pau, S., 257, 258, 260

Pavanello, M., 310

Pavone, E., 177

Pecoriello, R., 289, 306

Pedote, P., 94

Pedrale, R., 256

Pedrazzani, C., 204, 205

Pelaggi, D., 156, 157

Pernice, A., 285

Perone, M., 107, 172

Perotti, G., 43

Perrone, A., 294

Perrone, G., 163

Perrotta, M., 142

Perrotta, M.E., 204, 205

Perrucci, C., 270

Persechini, P., 330

Persiani, R., 214, 215, 216

Persico, M., 72, 74, 75, 114, 149

Perulli, A., 343, 345

Pettinari, D., 63, 64

Pezzoli, F., 19

Piaggio, F., 21

Piangatelli, C., 20

Piazza, A., 310

Piazzese, E., 108, 109, 110, 111, 112, 168, 170, 171, 321,322

Picchi, A., 292, 318

Piccin, O., 225

Piccione, E., 140

Piccirillo, R., 274

Piccoli, M., 102

Piero Guerrini, G., 12

Pilato, A., 350

Pilato, E., 1, 2, 9

Pilone, V., 128, 172

Pimentel, M., 323

Pinna, A., 30, 124, 206

Pinna, A.D., 9, 10, 17, 19, 29 , $34,279,284$

Piombino, P., 181, 182

Piquard, A., 82, 83

Piras, G.L., 11, 280

Pirozzi, F., 151

Pisapia, I., 35

Pisciaroli, A., 70

Piscitelli, V., 55, 61, 72, 116

Pizza, F., 119, 173, 174
Pizza, G., 281

Pizzio, M., 100

Pizzuto, O., 317

Poletto, G., 348

Poli, E., 92, 93, 94, 113

Polimeno, E., 293

Polistena, A., 42, 282

Pompeo, E., 315

Pontecorvi, A., 43

Pontoriero, D., 230, 231, 232, 233

Popovich, A., 340

Pozzi, S., 208

Pratesi, C., 337, 338, 339

Pratesi, G., 337, 339

Pressi, E., 85, 103

Princi, P., 43

Procaccini, E., 76

Procaccino, F., 76

Prosperini, A.P., 289

Puddu, A., 264

Pulli, R., 338, 339

Puma, F., 288

Puviani, L., 281

Quadrini, R., 238

Quaini, E., 3

Quartierini, G., 163

Quarto, G., 319, 320

Quattrone, C., 319, 320

Quercia, R., 307, 314

Raffaelli, M., 43

Ragni, F., 335, 336

Ragusa, M., 306

Raguso, M., 323

Ramacciato, G., 15, 16

Rampinelli, L., 163

Rampone, B., 205

Randone, B., 282

Ranieri, A., 228

Ranieri, V., 228

Rapicetta, C., 308

Raucci, A., 343, 344, 345

Rausei, S., 214, 215, 216

Rauso, R., 190

Rausos, R., 191

Ravaioli, M., 15, 16, 18, 19

Ravazzoni, F., 21

Raveglia, F., 308

Rea, F., 301, 312, 313

Refice, S., 4, 5, 6

Regolo, P., 201

Renzi, A., 126, 127

Ribacchi, F., 289

Riccardo, M., 310, 311

Ricci, G., 210

Ricciardi, W., 43

Ricciardiello, F., 223, 224

Ricco, G., 128
Rigante, M., 229

Rimonda, R., 164, 165

Rinaldi, R., 141

Rinaldo, A., 287

Risaliti, A., 13, 20, 23, 132

Risitano, G., 232

Rispoli, C., 114, 115, 116, 275

Rizzardi, G., 312, 313

Rizzello, G., 238, 239, 240, 241

Rizzetto, C., 312

Rizzo, G., 136

Roberta, P., 288

Robustelli, U., 32

Rocchetta, D., 192

Rocco, B., 323

Rocco, N., 114, 275, 276

Roina, G., 35

Roman, S., 301

Romano, A., 12, 52, 79, 80, 118, 177

Romano, M., 22

Rompianesi, G., 52, 118

Ronchi, P., 189, 193

Roncoroni, L., 101

Rondoletti, S., 212

Rosa, D., 234, 235, 236

Rosati, R., 153

Roscitano, A., 4, 6

Rossetti, G., 119, 173, 174

Rossetti, M.W., 228

Rossi, D.C., 196, 197

Rossi, P., 69, 278

Rossi, V., 245, 246, 247, 248, 249, 250, 251, 252, 253, 254

Rota, G., 142

Rottoli, M., 153

Roviello, F., 204, 205

Rozzanigo, U., 291

Rubino, C., 255, 264

Ruggiero, L., 92, 93, 113

Ruggiero, R., 45, 76, 91, 277

Rulli, F., 46, 47, 48, 49, 90

Russo, A., 235

Russo, E., 135, 136

Russo, F., 173, 174

Sacco, R., 96, 97, 106

Sagnelli, C., 119, 173, 174

Saint-Marc, O., 82, 83

Saltarel, A., 188

Salvatori, M., 43

Salvi, R., 298, 299

Salvucci, A., 31, 150

Samorani, D., 272

Sandomenico, F., 183

Sanna, A., 120

Sanna, S., 302, 303

Sannini, U., 311

Sannino, L., 237

Santanelli, F., 263
Santangelo, M., 138, 139

Santaniello, W., 14

Santella, A., 177, 185

Santini, D., 206

Santini, M., 293, 294

Sarli, L., 101

Sartori, A., 121, 313

Sassatelli, R., 52

Savini, C., 1

Scalise, C., 324, 325

Scalseggi, S., 269

Scandroglio, I., 144, 270

Scardino, E., 323

Scarpa, A., 223, 224

Scassa, F., 84, 85, 103, 104, 217

Scatizzi, M., 155

Schettino, M., 126, 127

Schiano di Visconte, M., 120

Schiavini, A., 291

Schiavon, M., 301, 313

Sciamannini, M., 288, 289, 306

Sciaudone, G., 122, 123

Sciuchetti, J.F., 7, 8

Sclafani, G., 5

Scuderi, E., 166

Scuderi, N., 261

Scuderi, V., 14, 22

Sellitri, F., 290

Selvaggi, F., 122, 123

Semeraro, A., 288, 289, 306

Sequino, G., 223, 224, 226

Serra, G.C., 212

Setacci, C., 341, 342, 343, 344, 345

Setacci, F., 341, 342, 343, 344, 345,346

Setaro, R., 91

Severi, S., 30, 124, 206

Sforza, C., 192

Siani, A., 328, 329

Siani, L.M., 329

Sicoli, F., 14

Signorelli, F., 201, 202, 203

Signorile, M., 92, 113

Silvestro, A., 237

Simon, C., 4

Sinatra, R., 4, 5, 6

Sirignano, P., 341, 342, 344

Sirri, L., 19

Smerieri, N., 52, 118

Solari, D., 198

Solfrini, G., 220, 221, 222

Sollitto, F., 285, 307

Sorrentino, D., 319, 320

Sorrentino, P., 54, 55, 60, 61, $62,72,73,125,351$

Sottile, R.G., 62

Spada, E., 314, 317

Spaggiari, M., 12, 52, 79, 118 
Sparavigna, L., 40

Spaziani, A., 147, 148, 167

Spaziante, R., 196

Speciale, R., 191

Sperlongano, P., 86, 87, 88, 273

Spiezia, F., 240, 242

Spiezia, S., 138, 139

Spilimbergo, I., 295

Spina, I., 338

Spinelli, F., 349

Spinosa, G., 138, 139

Spinzia, A., 181, 182

Spiritoso, R., 18

Spotti, S., 189, 193

Spreafico, A., 68

Spreafico, L.P., 244

Squillacciotti, S., 326, 327

Stanzione, F., 81, 154

Stefani, F., 197

Stefoni, S., 9, 10

Stella, F., 290

Suozzi, M.F., 234

Tacconi, F., 315

Taglialatela Scafati, S., 32

Tagliani, A., 291

Tancredi, G., 57

Tanzini, G., 71

Tarantini, S., 350

Tassi, V., 288

Tatti, A., 44

Tauceri, F., 218, 219, 220, 221, 222

Taurchini, M., 302, 303

Tavernini, T., 233
Tedesco, G., 136, 137

Tenconi, S., 308, 316

Terenzi, V., 194

Terzi, A., 295

Testini, M., 92, 93, 94, 113

Tirindelli, M.C., 239

Tolone, S., 119, 173, 174

Tomba, M.D., 3

Tommasino, G., 56, 57, 58, 59

Tonelli, E., 5

Torcasio, A., 95, 211

Torelli, F., 126, 127

Torre, M., 287

Tossani, E., 19

Tota, F., 307, 317

Tovecci, F., 54, 55, 60, 61, 62, $72,73,125,351$

Tozzi, U., 181

Trabattoni, P., 334

Tramontano, S., 128, 172

Tramonte, F., 120

Trapani, V., 129, 130, 131

Trecca, A., 66, 67

Trevisan, D., 332, 333, 352

Trevisan, G., 80, 121

Trignano, E., 283

Troise, G., 3

Troisi, N., 337, 338

Trombetti, A., 151

Tschabitscher, M., 229

Tshomba, Y., 347

Tsivian, M., 11, 280, 281

Tuci, F., 15, 16, 18

Turci, S., 2
Ugolini, G., 71

Urbani, M., 288

Usai, A., 24, 25, 26, 27, 28

Vadalà, G., 239, 240

Vagliasindi, A., 218, 219, 220, 221, 222

Valduga, P., 133

Valente, M., 265, 268

Valente, U., 21

Valentini, M.P., 92, 93, 113

Valentini, V., 175, 184, 194

Valitutti, P., 53

Valli, C., 270

Valsecchi, S., 189

Varotti, G., 19

Vavassori, A., 323

Vecchi, A., 13, 20, 23, 132

Vecchione, D., 198

Venetucci, P., 81

Ventucci, E., 187

Verdecchia, G.M., 218, 219, 220, 221, 222

Verga, M., 257, 258, 260

Verna, G., 256

Veroux, M., 133

Verweij, F., 323

Vetrano, E., 228

Vetrone, G., 15, 16, 18, 19

Vicidomini, A., 191

Vicidomini, G., 293, 294

Vigliaroli, L., 227

Vignudelli, A., 19

Villa, E., 3

Villa, M., 90
Villamaina, E., 271

Villari, S.A., 41, 83, 99, 134

Villoresi, P., 301

Vincenti, P., 23

Vincenzi, P., 13, 20, 132

Vindigni, A., 41, 99, 134

Vindingi, A., 83

Visconti, D., 165

Viti, A., 292, 318

Vivarelli, M., 15, 16, 18, 19

Voltolini, L., 308, 316

Weber, M., 279

Zaccaria, A., 298, 299

Zagami, M.G., 243

Zanatta, L., 312

Zanconati, F., 80

Zanello, M., 15, 16, 18, 19

Zanfi, C., 15, 16, 17, 18, 19

Zani, B., 133

Zannini, P., 296, 297

Zappia, R., 195

Zeccardo, E., 223

Zingarelli, E., 257, 258, 260

Zoccali, M., 214, 215, 216

Zocco, G., 104

Zona, G., 195, 197

Zuccaro, M., 135, 136, 137, 138, 139

Zuellig, R., 279

Zuin, A., 313 